\title{
Restoration of Coherent Images
}

Claudius Zelenka, M.Sc.

\author{
Dissertation \\ zur Erlangung des akademischen Grades \\ Doktor der Ingenieurwissenschaften \\ (Dr.-Ing.) \\ der Technischen Fakultät \\ der Christian-Albrechts-Universität zu Kiel \\ eingereicht im Jahr 2018
}


Kiel Computer Science Series (KCSS) 2018/3 dated 2018-12-13.

URN:NBN urn:nbn:de:gbv:8:1-zs-00000347-a2

ISSN 2193-6781 (print version)

ISSN 2194-6639 (electronic version)

Electronic version, updates, errata available via https://www.informatik. uni-kiel.de/kcss

The author can be contacted via cze@informatik. uni-kiel.de

Published by the Department of Computer Science, Kiel University

Multimedia Information Processing Group

Please cite as:

$\triangleright$ Claudius Zelenka. Restoration of Coherent Images Number 2018/3 in Kiel Computer Science Series. Department of Computer Science, 2018. Dissertation, Faculty of Engineering, Kiel University.

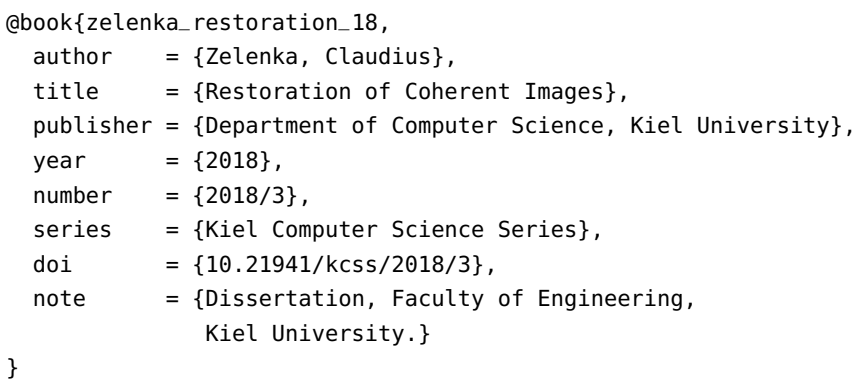

(C) 2018 by Claudius Zelenka 


\section{About this Series}

The Kiel Computer Science Series (KCSS) covers dissertations, habilitation theses, lecture notes, textbooks, surveys, collections, handbooks, etc. written at the Department of Computer Science at Kiel University. It was initiated in 2011 to support authors in the dissemination of their work in electronic and printed form, without restricting their rights to their work. The series provides a unified appearance and aims at high-quality typography. The KCSS is an open access series; all series titles are electronically available free of charge at the department's website. In addition, authors are encouraged to make printed copies available at a reasonable price, typically with a print-on-demand service.

Please visit http://www. informatik. uni-kiel.de/kcss for more information, for instructions how to publish in the KCSS, and for access to all existing publications. 
1. Gutachter: Prof. Dr.-Ing. Reinhard Koch Christian-Albrechts-Universität Kiel

2. Gutachter: Prof. Dr. Dirk Nowotka Christian-Albrechts-Universität Kiel

Datum der mündlichen Prüfung: 9. November 2018 


\section{Zusammenfassung}

In dieser Thesis werden mehrere neue Algorithmen für eine qualitativ hochwertige Restaurierung von kohärenten Bildern vorgestellt. Diese Aufgabe kann mit den bekannten Methoden für die Restaurierung von nicht kohärenten Bildern nicht gelöst werden. Die neuen Algorithmen sind auf die Wiederherstellung von Bildern in kohärenten Abbildungssystemen, bei denen die Aberrationen a-priori bekannt sind, ausgerichtet. Sie dienen der Korrektur der Wellenfront und erreichen eine wesentlich höhere Qualität der Bildrekonstruktion als sämtliche vorbekannten Verfahren.

Die Algorithmen in dieser Thesis basieren auf neuesten Optimierungsalgorithmen, wie Projektionen in konvexe Sets, proximale Optimierung und fraktaler Ähnlichkeit. Die Konvergenz und Leistung der einzelnen Algorithmen wird ausführlich in unterschiedlichen Szenarien mit simulierten und realen Bildern untersucht. Eine praktische Erprobung der neuen Algorithmen an mikroskopischen Aufnahmen von unterschiedlichen biologischen und humanen Proben, wie auch an Aufnahmen vom Shadowgraph, bestätigt ihre Effizienz. Diese wird weiter bestätigt durch die Restaurierung von Shadowgraph-Aufnahmen, die eine Expedition in der Chesapeake Bay vor der Küste von Virginia im Atlantischen Ozean erstellt hat.

Bei dem fokussierten Shadowgraph, der eine breite Anwendung bei der Beobachtung von Plankton im Wasser findet, können die neuen Algorithmen durch eine Vergrößerung der Fokustiefe einen wesentlichen Vorteil bringen. Eine automatische Erkennung der optimalen Schärfeebene auf der Grundlage von "deep learning"und "convolutional neural networks" wird entwickelt und weiterverwendet zur Darstellung von ",all-in-focus“ Bildern. Dadurch wird die praktische Anwendung der Restaurierung von kohärenten Bildern wesentlich vereinfacht. Eine innovative Berechnung der Tiefe vom Defokus und eine 3D-Darstellung von Objekten, die sich in unterschiedlichen Entfernungen im Einzelbild befinden, wird vorgestellt. Diese neue Art der Präsentation ermöglicht eine leichte Auswertung des 
betrachteten Volumen.

Die neuen Algorithmen haben vielversprechende künftige Anwendungen, auch in anderen Gebieten, z.B. in der adaptiven Optik und der Astronomie. 


\section{Abstract}

In this thesis a series of novel algorithms for high quality restoration of coherent images is introduced. This task cannot be solved with established methods for the restoration of incoherent images. These algorithms focus on the correction of images in coherent imaging systems with a-priori known aberrations. The new wavefront correction algorithms achieve a significantly higher restoration quality than any previously known technique.

The algorithms in this thesis are based on latest advances in optimization algorithms, particularly projections onto convex sets, proximal optimization and fractal self-similarity. Convergence and performance of the individual algorithms are analyzed in detail in various scenarios on real and simulated images. The evaluation also deals with the impact of noise on the restoration quality. Practical application of the new algorithms on microscopic images of diverse biological and human samples, as well as shadowgraph images of plankton acquired with a laboratory setup prove their efficiency. This is further confirmed by results on shadowgraph images acquired in a real expedition setting in the bay of Chesapeake Bay in the Atlantic of the coast of Virginia.

The focused shadowgraph, which is widely used in underwater plankton observation can greatly benefit from large extension of the depth of field made possible by wavefront correction. We present an automatic estimation of the best focus using deep learning and convolutional neural networks for all-in-focus images. This is a major improvement in practical application of the novel coherent images restoration. An innovative computation of depth from defocus is used for 3D visualization of objects acquired at different focus distances on a single image. This novel method of visualization allows an easy survey of the observation volume.

The new algorithms also have promising future applications in other areas, for example in adaptive optics, astronomy and confocal microscopy. 



\section{Acknowledgements}

Firstly, I would like to thank Prof. Dr.-Ing. Reinhard Koch for his motivation, patience and excellent supervision of my research and thesis. His insightful comments have significantly guided and improved my work. I very much appreciate his support in every regard.

I would like to thank Prof. Dr. Dirk Nowotka, the second reviewer, who has also been a member of the thesis committee. Further, I want to express my gratitude to Prof. Dr. Reinhard von Hanxleden, the chairman of the thesis committee, and Prof. Dr. Lorenz Kienle, member of the thesis committee.

I would like to thank my current colleagues (Dr.-Ing. Arne Petersen, Johannes Brünger, Luca Palmieri, Renate Stäcker, Dr.-Ing. Sandro Esquivel, Sascha Clausen, Simon-Martin Schröder, Stefan Reinhold, Tim Michels, Tobias Schwede, Torge Storm, Yu Tang and Yuan Gao) and also my former colleagues (Dr.-Ing. Andreas Jordt, Dr.-Ing. Anne Jordt, Dr.-Ing. Daniel Jung, Dr.-Ing. Dominik Wolters, Falko Kellner, Matthias Kardel and Dr.Ing. Oliver Fleischmann). My colleagues in the Multimedia Information Processing group are a friendly and creative team, where everyone motivates each other. My special thanks belong to Jan Taucher from GEOMAR Helmholtz Centre for Ocean Research Kiel for his stimulation of the shadowgraph project.

I am deeply grateful for funding from the German Research Foundation (DFG) Cluster of Excellence FUTURE OCEAN under proposals CP1331 and CP1525, by the Petersen-Foundation in Kiel under project 385 and by the Central Innovation Programme for SMEs of the Federal Ministry for Economic Affairs and Energy of Germany under grant agreement number 16KN044302.

Last but not least, I wish to express my thanks to my friends and family for their great support. 



\section{Contents}

1 Introduction 1

1.1 Motivation for Coherent Restoration . . . . . . . . . . 6

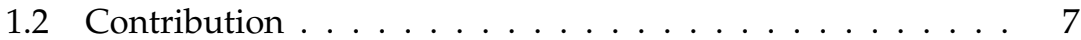

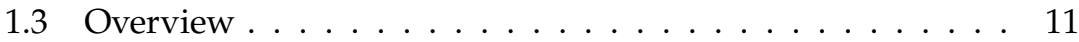

2 Related Work 13

2.1 Coherent Restoration . . . . . . . . . . . . . . . . . . . 13

2.2 Image Deconvolution . . . . . . . . . . . . . . . . . . . . . . 15

2.3 Phase Retrieval . . . . . . . . . . . . . . . . . . . . . . 17

2.4 The Focused Shadowgraph $\ldots \ldots \ldots \ldots \ldots$

3 The Wavefront Correction Algorithm 29

3.1 Description of the Algorithm . . . . . . . . . . . . . . . . . 29

3.1.1 Physical Introduction and WFC-GS . . . . . . . 33

3.2 Projection Algorithms . . . . . . . . . . . . . . . . 34

3.3 Convergence . . . . . . . . . . . . . . . . . . . 38

3.4 The WFC-FISTA Algorithm . . . . . . . . . . . . 40

3.5 Internal Patch Recurrence WFC . . . . . . . . . . . . . . . 44

3.6 Convergence Criteria . . . . . . . . . . . . . . . . . 47

3.7 Finding Optimal Aberration Parameters . . . . . . . . . . . . 49

3.8 Large Image Processing . . . . . . . . . . . . . . . . . 51

3.9 Boundary Ringing Suppression . . . . . . . . . . . . . . . 52

3.10 Deconvolution Algorithms on Coherent Images . . . . . . . 56

4 Applications and Results 59

4.1 Implementation . . . . . . . . . . . . . . . . . . . . 59

4.2 Results on Simulated Images . . . . . . . . . . . . . 60

4.2.1 Comparison with Related Work . . . . . . . . . . 66

4.2.2 Comparison and Analysis of WFC Algorithms . . . . 69

4.2 .3 Initialization . . . . . . . . . . . . . . . . . 74 
Contents

4.2.4 Imperfect Input . . . . . . . . . . . . . . . . . . . 77

4.2.5 Results of Large Image Processing . . . . . . . . . . . 84

4.2.6 Comparison of Boundary Ringing Suppression Techniques . . . . . . . . . . . . . . 86

4.2.7 Application of Incoherent Deconvolution on Coherent Images . . . . . . . . . . . . . . . . . . . . 89

4.3 Microscopic Images . . . . . . . . . . . . . . . . . . . . . . . 90

4.3 .1 Experimental Setting . . . . . . . . . . . . . . . 90

4.3 .2 Results . . . . . . . . . . . . . . . . . . . . . 94

4.4 Focused Shadowgraph . . . . . . . . . . . . . . . . . 97

4.4 .1 Image Filtering . . . . . . . . . . . . . . . . . . 106

4.4.2 WFC-Autofocus for Spatially Variant Blur . . . . . . 110

4.4.3 Image Sharpness Classification with Deep Learning 117

4.4.4 All-in-Focus Images and 3D View . . . . . . . . . 123

4.5 Shadowgraph in Marine Application . . . . . . . . . . . . . 128

5 Conclusion 133

5.1 Future Work . . . . . . . . . . . . . . . . . . . . . . . . 135

A Mathematical and Physical Background 141

A.1 Scalar Diffraction Theory . . . . . . . . . . . . . . . . 141

A.1.1 Huygens-Fresnel Principle . . . . . . . . . . . . . 142

A.1.2 Helmholtz Equation . . . . . . . . . . . . . . . . . . 142

A.1.3 Propagation of the Angular Spectrum . . . . . . . . . 144

A.1.4 Influence of a Lens . . . . . . . . . . . . . . . . . 149

A.2 Wavefront Focusing . . . . . . . . . . . . . 151

A.3 Coherence . . . . . . . . . . . . . . . . . . 155

A.4 Peak Signal to Noise Ratio . . . . . . . . . . . . . . . . . 159

A.5 Zernike Polynomials . . . . . . . . . . . . . . . . . . 160

A.6 Projections onto Convex Sets . . . . . . . . . . . . . . 164

A.7 FISTA . . . . . . . . . . . . . . . . . . . 166

A.8 Convolutional Neural Networks _ . . . . . . . . . . . . . . . . 169

A.8.1 Neuron . . . . . . . . . . . . . . . . . . . . . . . 169

A.8.2 MLP . . . . . . . . . . . . . . . . . 171

A.8.3 Convolutional Layers . . . . . . . . . . . . . . . 172

A.8.4 Training . . . . . . . . . . . . . . 174

xii 
Contents

$\begin{array}{ll}\text { Bibliography } & 177\end{array}$

$\begin{array}{lr}\text { List of Symbols } & 199\end{array}$

List of Symbols - POCS \& CNN 203

List of Symbols - Scalar Diffraction Theory 205

$\begin{array}{lr}\text { List of Abbreviations } & 207\end{array}$ 



\section{List of Figures}

2.1 Two images are shown in (a) and (b), with their phase information exchanged in the Fourier-space in (c) and (d). Clearly, the phase holds most of the structural image information (inspired by [Osh12]). . . . . . . . . . . . . . 18

2.2 Optical system for phase retrieval with aperture plane $A$ and image plane $0 . f$ denotes the focal length of the lens. . 19

2.3 The Gerchberg-Saxton algorithm. . . . . . . . . . . . . . . 24

2.4 Optical drawing of the focused shadowgraph. Black dots show the illumination edge rays, blue the rays from the objects projection onto the camera sensor. Shown is an adaptation of a drawing from [Set01]. . . . . . . . . . . . 26

3.1 Wavefront correction in an out of focus optical system. Compared to Figure 2.2, the image plane is out of focus, which causes an additional spherical wavefront deformation. The wavefront deformation is visualized as a surface of points with equal phase across the $x-y$ coordinates of the aperture plane.

3.2 Overview of the WFC-GS algorithm. . . . . . . . . . 35

3.3 WFC-GS algorithm iterations. The planes are arranged as in Figure 3.1. The numbers resemble the same steps as in Figure 3.2.

3.4 Non-convexity of the image plane constraint. The circle show the set of points with identical amplitude and the arrows highlight the phase of two examples. The red connecting line is their linear combination. . . . . . . . . . 40

3.5 The same blurred image at different scales. The right image is sharper, because the width of the blur effects is scaled together with the image. . . . . . . . . . . . 45 
3.6 Runtime for per iteration WFC-GS for a square image with increasing width and height (x-axis). . . . . . . . . . . . 52

3.7 Example of image restoration with strong boundary ringing, produced by restoring a small defocused image with $256 \times$ 256 pixels. The input image was created by cropping a larger image with simulated defocus. For more details, see Section 4.2.6. . . . . . . . . . . . . . . . . 54

3.8 Illustration of replicate, cyclic and symmetric boundary condition with the original image in the middle and the new padded image within the red border. . . . . . . . . . 55

4.1 Defocus of increasing strength applied on an image, measured in maximum wavefront modulation in wavelengths. . 60

4.2 Visualization of wavefront deformation in multiples of wavelength over the $x-y$ aperture plane. . . . . . . . . . . . 62

4.3 Image with different wavefront deformations. The visible wraparound in image (b) is due to the very strong asymmetric deformation and the repetitiveness of the Fourier transform. . . . . . . . . . . . . 63

4.4 Symmetry of defocus. . . . . . . . . . . . . . . . . . 64

4.5 The Wavefront Correction Algorithm restores images disturbed by strong defocus. . . . . . . . . . . . . . . 65

4.6 Correction of input images with increasing defocus in Figure $4.1 \ldots \ldots \ldots \ldots \ldots$. . . . . . . . . . 66

4.7 Different images with simulated aberrations and restoration with 600 iterations of WFC-HAAR. . . . . . . . . . . . . 67

4.8 Image restoration with wavefront correction and with related works inverse filter method. . . . . . . . . . . . 68

4.9 Comparison of the convergence of different WFC algorithms in $d B$ of PSNR. . . . . . . . . . . . . . . 70

4.10 Comparison at iteration 7 of restoration quality between different WFC algorithms. . . . . . . . . . . . . . 71

4.11 Restorations with limited iterations. Results after 3, 5, 15, 25, 100 and 500 iterations are shown. For the input image see Figure $4.8 \mathrm{~b}$. 
4.12 Enlarged image detail for a visual comparison of the restoration with different algorithms. To highlight differences all images are restorations with only 10 iterations.

4.13 Speed comparison of the different WFC-algorithms in seconds per 500 iterations. The WFC prefix is omitted in the labels to save space. The WFC-SS algorithm is not included, because it does not fit the chart. . . . . . . . . . . . . . 75

4.14 Visual comparison initialization strategies. MI stands measured initialization, $\mathrm{RI}$ is random initialization and ZI stands for zero initialization, details see text. For input images, see Figure $4.8 \mathrm{~b} . \ldots \ldots \ldots \ldots \ldots \ldots$. . . . . . . . 76

4.15 Comparison of different initialization strategies by their convergence. Show is the PSNR ratio per iteration against the undisturbed image. . . . . . . . . . . . . . 78

4.16 Restoration quality comparison for a given number of iterations by PSNR for different algorithms with a slightly wrong corrective wavefront with $2 \%$ radius. . . . . . . . 79

4.17 Defocused image with Gaussian noise. . . . . . . . . . . 79

4.18 Comparison of algorithms in restoration of noisy images. . 80

4.19 Comparison of restoration with very strong noise. Wavefront correction with self-similarity prior can cope with this level of noise.

4.20 Comparing the results of the best algorithm for weaker noise WFC-AP and WFC-FISTA with WFC-SS for strong noise. . . . . . . . . . . . . . . . 83

4.21 Comparison of result with tiling and non-tiling CPU WFC$\mathrm{AP}$ algorithm restorations. The sharpness of the restoration can be seen best on the eye pupils of the left person in the magnification. The tiling approach exhibits boundary artifacts on the tile borders. . . . . . . . . . . . . . 85

4.22 Ground truth and input for boundary ringing evaluation. . 86

4.23 Input images for different padding strategies after half image width padding is applied. . . . . . . . . . . . . 87

4.24 Visible difference of different boundary ringing suppression techniques. A large difference between all result images can be seen in the visibility of streaks on the left side of the image. 89 
4.25 Restoration results on Image 4.25a with incoherent deconvolution algorithms, Richardson-Lucy deconvolution [Ric72] [Luc74] and fast image deconvolution using hyper-laplacian priors [KF09]. Image $4.25 \mathrm{~d}$ show the result with the novel WFC algorithm.

4.26 Schematic drawing of the optical path in microscopic application 1

4.27 Custom illumination and microscopic setting, a paper tube to reduce light scatter and environmental light is recommended.

4.28 Comparison of the sharp original, the measured defocused image, the synthetic defocus image and the restored image. 95

4.29 Comparison of different novel algorithms on a microscopic image of a test target. . . . . . . . . . . . 96

4.30 Image restoration of a image of a human kidney slice. The prominent dark streaks are blood vessels inside the kidney. The restoration makes them visible. . . . . . . . . . . . . 98

4.31 Restoration of a pigeon blood sample. The dark ellipses are individual blood cells.

4.32 Restoration of a human neuron sample. The dark structure is a neuron. Dark dots are cell cores. Only slight defocus, imperfect restoration due to noisy background and 3D structure of the neuron. . . . . . . . . . . . . . . . . . 100

4.33 Drawing of the plankton shadowgraph system. Optical rays as in Figure 2.4 are omitted for clarity. . . . . . . . . . . 102

4.34 The shadowgraph laboratory setting with cuvette target. The light path is illustrated in yellow . . . . . . . . . . 102

4.35 Shadowgraph with water tank . . . . . . . . . . . . . . 103

4.36 Comparison of illumination sources. . . . . . . . . . . . . 103

4.37 Comparison between sharp image of the shadowgraph (a), defocused image (b) and restoration with WCF-GS algorithm (c). . . . . . . . . . . . . . . . . . . . . . 105

4.38 Comparison of WFC-GS and WFC-AP restoration. . . . . . . 106 
4.39 Logarithmic absolute of the DCT-spectrum from the restoration in Figure 4.38a. Horizontal spatial frequencies in $\mathrm{x}-$ coordinates, vertical frequencies in $y$-coordinates. The origin is in the top left corner. . . . . . . . . . . . . . . . . 107

4.40 Plot of the chirp function used as a frequency filter with absolute $c_{\text {filter }}$ values on the y-axis across the 512 datapoints. 108

4.41 DCT of 2D-chirp function and multiplication of chirp and restoration. Horizontal spatial frequencies in x-coordinates, vertical frequencies in $y$-coordinates. The origin is in the top left corner. . . . . . . . . . . . . . . . . . . . . 109

4.42 Filtering result of WFC-GS and WFC-AP. . . . . . . . . . 109

4.43 Artificial test image with spatially variant defocus. The top left partial image is sharp, the defocus is increasing by row and column. . . . . . . . . . . . . . . . . . . 111

4.44 Restoration stack of simulated image with spatially variant blur only 6 are shown as demonstration.

4.45 Restoration stack with increasing defocus correction on shadowgraph image with spatially variant blur, only 6 images of 60 are shown. . . . . . . . . . . . . . . . . . 114

4.46 Shadowgraph image for sharpness criterion evaluation. . . . 115

4.47 Image sharpness as measured per criterion for restorations of the selected image patch (Figure 4.46) with different wavefront aberration estimations. . . . . . . . . . 116

4.48 Training images generation with a region of interest throughout a focal stack of restoration with increasing defocus aberration correction. Image 4.48a is from the original image, while image $4.48 \mathrm{f}$ is the sharpest image and is sorted to the sharp image training examples. All other images are unsharp and are used as training examples for the unsharp class. . . . . . . . . . . . . . . . . . . . . . . 118

4.49 Sharp and unsharp examples from the training set . . . . 119

4.50 Learning rate decay in three steps from 0.01 to 0.0001 . . . . 121

4.51 Network training graph on Caffenet . . . . . . . . . . . . 121

4.52 Network training graph with Googlenet. . . . . . . . . . . 122

4.53 Network training graph with Resnet 32. . . . . . . . . 123 
4.54 Comparison of $\mathrm{CNN}$ architectures, visualized with automated layouting, scaled to fit. In cyan: Input data layer, in red: Convolution or fully connected layers, in yellow: Activation layers, in blue: pooling layers, in orange: Flattening/concatenation layers, in pink: Normalization, dropout and loss layers. . . . . . . . . . . . . . . . . . . . . 124

4.55 Simulated all-in-focus image. . . . . . . . . . . . . . 125

4.56 Comparison of shadowgraph and all-in-focus image. . . . 127

4.57 3D visualization of depth from defocus. . . . . . . . . . . . 128

4.58 All-in-focus and 3D visualization of shadowgraph images. . 129

4.59 Example images captured with a shadowgraph in Chesapeake Bay from [Bi15]. The blue rectangles highlight the

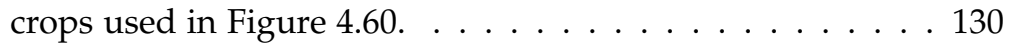

4.60 Cropped segments of images $4.59 \mathrm{a}$ and $4.59 \mathrm{c}$ with restoration. 132

4.61 Dark and noisy input image, refocusing on two plankton objects. . . . . . . . . . . . . . . . . 132

5.1 Schematic of adaptive optics setup, such as a telescope. An Incoming wavefront is reflected on a deformable mirror, split on a beam splitter, then observed by wavefront sensor and camera. The system is controlled by an acquisition computer and an adaptive optics (AO) controller, compare [Mer88].

A.1 A spherical thick lens can be seen as a composition of three parts, adaptation from [Ers06].

A.2 An incoming wavefront is incident on a semitransparent object. The propagated wavefront is focused with a lens into the focal plane, adapted from [Goo05]. . . . . . . . . . . 152

A.3 Spatially and temporally coherent light from an incoherent light source ${ }^{1}$.

A.4 Illustration for spatial coherence calculations. Adapted from: [RP89][Bre17]. . . . . . . . . . . . . . . . . . . . . 158

A.5 Visualization of wavefront shapes. . . . . . . . . . . . . 160

A.6 Definition of $\mathrm{p}$ and $\theta$ for the Zernike polynomial (adapted from [GW06]). 
A.7 Color-coded visualization of the Zernike polynomials in a pyramid with high values in blue and low values in yellow.

Created with Matlab code by [TSS11] and [STS+13]. . . . . . 163

A.8 Behavior of different activation functions. . . . . . . . . . . 170

A.9 Drawing of a neuron. . . . . . . . . . . . . . . 171

A.10 Fully connected layers of neurons. . . . . . . . . . . . 172

A.11 Convolution resulting in 3D feature maps. . . . . . . . 173

A.12 A convolutional neuron network with two convolutional layers with a pooling layer in between, followed by two fully connected layers. . . . . . . . . . . . . . . . . . . 174

A.13 Maximum pooling with a $2 \times 2$ block. . . . . . . . 176 



\section{List of Tables}

1.1 Comparison of related works and WFC. . . . . . . . . 9

1.2 Coherent image restoration algorithms used in this thesis. . 10

4.1 Restoration quality measurement by peak signal to noise ratio (PSNR) in $\mathrm{dB}$ of different boundary ringing suppression techniques. The algorithm used is WFC-AP with 50

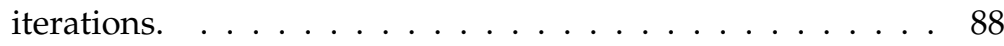

A.1 Zernike polynomials with mode index by [Nol76] showing the relationship with Seidel wavefront aberrations. . . . . . 162 



\section{Chapter 1}

\section{Introduction}

Image restoration is a very important task in the image processing with numerous applications. The typical application consists of the correction of optical errors in non-coherent imaging systems, like standard photography. These optical errors often manifest themselves as blur, therefore a restoration of distorted images is usually termed as deblurring. Non-coherent deblurring is a well-established field and many algorithm are known, we look closer at these in the Related works Section 2.2.

This thesis deals with a different task, namely image restoration in a coherent imaging system. The image formation theory of these two imaging methods differs, as a consequence incoherent algorithms work poorly on coherent images.

Light sources with lasers and LEDs have opened new applications in microscopy, which stimulates research on the prospects of coherent imaging. It will be shown, that the conditions for coherent imaging are easily achieved in an optical microscope with low cost LED illumination. Another application, which provides coherent images, is the focused shadowgraph. The differences between coherent and incoherent imaging and the resulting consequence are discussed in Section 1.1.

The main research question of this thesis is: How can high quality image restoration be achieved for coherent systems? To this effect, in this thesis a group of algorithms based on the novel Wavefront correction principle is introduced. The proposed algorithms are the first algorithms, succeeding in proper restoration of coherent images. Prior work on restoration of coherent images did not achieve sufficient quality.

They will be practically tested on two fields of application, microscopy and underwater shadowgraph imaging for plankton observation both in laboratory and in oceanic expedition conditions. 


\section{Introduction}

These new algorithms, the wavefront correction algorithms (WFC), can be applied directly on imagery from coherent microscopy and deal with the restoration of images blurred by wavefront errors, caused frequently by imperfect optical elements, alignment or by defocus. A very essential practical contribution of these algorithms is the removal of stringent requirements on the optical quality in a diffraction limited imaging system. The limiting factor in microscopy are the aberrations of the microscope lens. High resolution requires a lens with a high numerical aperture, which unavoidably has higher aberrations.

A trade-off is needed between the numerical aperture and the acceptable lens aberration. Another limiting factor is the cost of complex high aperture lenses. The new algorithms have the capability to correct any optical aberration, including the defocus. This will be helpful especially in optical systems with high numerical aperture. Such microscopes have low depth of focus and therefore need frequent and precise adjustment. The new WFC algorithms allow the restoration of images blurred by lens aberrations, which is a great improvement in coherent microscopic imaging.

Because the aberrated image does not readily provide all the information of a sharp image (it lacks phase information), wavefront correction is algorithmic challenge. Note that this is the key difference to holography, which as explained by [KS10] 'is a record of interference between light diffracted from object illuminated by a coherent light and a known reference beam' and 'contains both the amplitude and phase of the diffracted wave'.

The algorithms work on the common novel principle of projecting the aberrated image into a virtual focus plane, where image sharpness through the phase conditions is enforced, which leads iteratively to a restored sharp image. Imperfections in the coherent optical system, including defocus can be described as wavefront deformation.

In the development of the wavefront correction algorithm the assumption is made that the wavefront deformation is known a-priori. This means that it has to be measured or estimated in some way. The wavefront distortion for example of microscopic lenses or astronomic mirrors can be easily measured with established methods. The wavefront distortion of most optical components will usually not change much over time, however the 
focus may change. Hence, special attention is paid to defocus correction.

The type and strength of the aberration that has to be compensated is typically predetermined. For the application on shadowgraph images we present a useful extension of our restoration method with automatic search for optimum focus, which can be applied also in the microscopy. This approach can be generally used for automatic image restoration without a prior known aberration values.

In this thesis a family of wavefront correction algorithms is developed. They are all based on the physics of light, which can be quite accurately described by the scalar diffraction theory. First the idea of restoration process is presented and implemented with a basic algorithm WFC-GS. In the following more advanced algorithms are developed and tested on real and simulated images. These different formulations of wavefront correction are based on recent advances in mathematics and phase retrieval, and result in a number of improved wavefront correction algorithms. However it should be pointed out, that the basic algorithm already delivers useful results. Using the basic algorithm as a starting point, these advanced algorithm are developed.

As the next algorithm, the wavefront correction Hybrid-Input-Output (WFC-HIO) algorithm is defined, inspired by the Hybrid-Input-Output algorithm for phase retrieval [Fie82]. Phase retrieval is an established algorithmic field in the field of coherent optics. Following this link allows a generalized mathematical formulation of the wavefront correction as a problem of projections onto non-convex sets and gives way to apply modern mathematics. More projection based wavefront correction algorithms are derived from this connection namely WFC-AP and WFC-RAAR and we compare their performance to the other algorithms. An overview of the algorithms is given in Section 1.2.

Most recent advances in the field of projections onto convex sets (POCS) have been implemented in the Haugazeau alternating averages reflections algorithm [BCL06] [BK15]. Though this algorithm provides strong convergence properties for convex sets only, a condition not satisfied by the wavefront correction problem, as will be shown in Section 3.3, it shows stable performance on real and simulated images and allows the formulation of the fastest algorithm in this thesis, the WFC-HAAR.

The problem of wavefront correction can essentially be identified as an 


\section{Introduction}

optimization problem with objective function and secondary conditions. Hence adding total variation regularization and a special, novel gradient approximation we can apply variable splitting and FISTA (Fast Iterative Shrinkage Thresholding Algorithm) [BT09b]. Furthermore, using the fractal self-similarity property of natural sharp images we define an algorithm especially well suited for noisy images.

The performance of all algorithms will be explored on simulated data and on real images in two different fields of application. Coherent microscopy is an important field of research and could greatly benefit from the correction of blur caused by an imperfect optical system. Images of coherent microscopy are also used as a benchmark to compare the practical properties of different wavefront correction algorithms. We use standard test targets as well as real biological and medical tissue samples for a realistic practical evaluation. An important part of this thesis deals with the comparison with existing prior methods, which as we will show do not provide adequate results.

As mentioned above, the second field of application is the focused shadowgraph. Our laboratory shadowgraph is a plankton observation instrument, which is developed in a cooperation project between GEOMAR Helmholtz Center for Ocean Research Kiel and Kiel University. An introduction into the shadowgraph and related work is given in Section 2.4.

The shadowgraph is of considerable interest, because it allows unique insights into marine life. The oceans cover the largest part of earth's surface. While the ocean ground is only sparsely explored, on the volume of the oceans there is even less available information. In-situ measurement are especially difficult to execute. The goal of this project is the development of an instrument that allows to observe the abundance and variation of plankton in different water depths. This data can provide a basis for better understanding of biomass transport and of the marine life cycle in general. It is known that plankton distribution is subject to many environmental factors, however many species living in the ocean may still be undiscovered. Better knowledge of plankton life can help in a better understanding of ocean circulation and future changes in the ocean which may be caused by global warming.

Other solutions besides the shadowgraph include collecting water samples from different depths. However this approach is unpractical due 
to the fact that plankton occurs generally in low concentration. Therefore, this project aims for an optical in situ observation instrument that shall be capable of imaging plankton in volume with approx. $20 \mathrm{~cm}$ length.

The depth of focus of the shadowgraph camera is only $2-3 \mathrm{~cm}$, which is given by the required resolution. As we will show, application of the wavefront correction algorithm expands the useful observation range at least by factor 7 . Otherwise, focus stacking, a technique in which a series of images with different focus value is taken and combined, could be used, but this is difficult because the plankton is in constant motion. With the wavefront correction only one image is needed for in-depth restoration of the observed volume. Our approach of wavefront correction for the shadowgraph has been successfully applied on real shadowgraph images and also influences the design of the observation instrument and several pre and post processing steps. The task is an easy and automatic evaluation of an extended volume of $8.7 \mathrm{~cm} \times 5.8 \mathrm{~cm} \times 20 \mathrm{~cm}$. Therefore we developed a novel automatic defocus parameter estimation, akin to autofocus, which allows automatically selecting the correct plane of focus for individual plankton objects.

Existing methods for defocus estimation, which are used in many incoherent imaging systems, showed unsatisfactory performance on coherent images, as will be discussed in Chapter 4.4.2. The novel defocus estimation, which relies on deep learning and convolutional neural networks, is introduced in the Appendix in Section A.8. The restored spatially variant images are visualized with a novel approach as all-in-focus images. Furthermore, a novel three dimensional depth from defocus image of the corrected objects is presented.

Practical testing of the algorithms has been done with a laboratory version of a focused shadowgraph, which is described in Section 4.4. The shadowgraph is developed together with GEOMAR Helmholtz Center for Ocean Research Kiel. To evaluate the efficiency of the algorithms, it is important to see how they perform in real non-laboratory conditions. We could not use images from the shadowgraph developed together with GEOMAR Helmholtz Center for Ocean Research Kiel, because it has not yet been practically deployed. Hence, we use for the evaluation shadowgraph images by [BGB+15] acquired in the Chesapeake Bay off the coast of Virginia. The restoration of these oceanic images is shown in 


\section{Introduction}

Section 4.5.

Moreover, it will be shown how wavefront correction greatly improves the performance of the focused shadowgraph by raising the focal range up to sevenfold. This is a major enhancement of the shadowgraph as an observation instrument.

\subsection{Motivation for Coherent Restoration}

Image restoration is one of main challenges of image processing and is widely used in computer vision, astronomy, microscopy, and in the evaluation of airborne or satellite images. It is a very active field and a large number of image restoration methods has been developed and optimized for dedicated applications in the last decades.

The goal of image restoration is to restore an undisturbed original image from the observed image. This image is influenced by disturbances, which can be modeled with a convolution kernel and additional noise. The influence of the convolution kernel is commonly a blur effect, therefore we also call it blur kernel. This imaging model for incoherent systems is defined by

$$
O_{S I}=I_{i n c} \otimes B+N,
$$

where $O_{S I}$ is the observed intensity image that is formed by the convolution $\otimes$ of the undisturbed image intensity distribution $I_{\text {inc }}$ with a blur kernel $B$, and an additive noise term $N$. Applied to an optical system the blur kernel resembles a point spread function (PSF). The goal of image restoration algorithms is the recovery of the undisturbed $I_{i n c}$, given $O_{S I}$ and $N$.

One can regard the problem of inverting the influence of this blur kernel as a deconvolution. Therefore this subcategory of image restoration algorithms is known as deconvolution algorithms. All general incoherent image deconvolution methods rely on the blur model in equation 1.1.1.

Widely used are so called non-blind deconvolution algorithms, which require prior knowledge of the blur kernel $B$. In contrast, if the kernel is estimated from the observed image itself, the algorithms are called blind deconvolution algorithms. The standard deconvolution methods 
work directly on image data recorded using an image sensor. This data is intensity based and these algorithms assume incoherent illumination, following this model of image formation. An overview of deconvolution methods in given in Chapter 2.

Coherent image model In contrast to the intensity based incoherent image formation model, in coherent imaging the image formation model is based on amplitude. In the following a description of image formation as a linear filter is used. It is based on the scalar diffraction theory of Sommerfeld and more precisely the approximations of Fresnel diffraction. Detailed derivations can be found in [Goo05] and [BW80].

Let $U$ be the undisturbed complex amplitude distribution of a wavefield, then

$$
A_{m}=U \otimes B_{a}+N_{a},
$$

gives the amplitude of the resulting wavefield $A_{m}$, with $B_{a}$ the coherent complex blur kernel and $N_{a}$ an additional noise term. Intensity and amplitude are related, intensity is the amplitude squared:

$$
O_{S I}=\left\|A_{m}\right\|^{2} \text {. }
$$

Similarly the blur kernels are related:

$$
B=\left\|B_{a}\right\|^{2} .
$$

Clearly incoherent imaging is linear in intensity, while coherent imaging is linear in amplitude. Despite the seeming resemblance, a consequence of this difference is that incoherent imaging cannot model the interference phenomenons like wave extinction between adjacent waves, which occur in coherent imaging. Therefore traditional algorithms designed for incoherent images cannot be used for coherent images.

We will expand on this with a more detailed theoretical explanation, on why incoherent deconvolution algorithm fail in Section 3.10, when deconvolution algorithms as well as more optic terms have been introduced.

\subsection{Contribution}

Parts of this thesis have already appeared in the following publications: 
$\triangleright$ Claudius Zelenka and Reinhard Koch, "Improved wavefront correction for coherent image restoration", Opt. Express 25, Issue 16, 18797-18816 (2017), [ZK17].

$\triangleright$ Claudius Zelenka and Reinhard Koch, "Restoration of images with wavefront aberrations", oral paper at 2016 23rd International Conference on Pattern Recognition (ICPR), Cancun, 2016, pp. 1388-1393, IEEE [ZK16].

$\triangleright$ Claudius Zelenka and Reinhard Koch, "Restoration of Images with Wavefront Aberrations", arXiv preprint, arXiv:1704.00331, Apr. 2017. (preprint of ICPR 2016 publication).

$\triangleright$ Claudius Zelenka, "Schnelles Bildkorrekturverfahren für eine vereinfachte adaptive Optik", European patent application EP 16178151.3, PCT patent application PCT/DE2017/100497, Priority 06.07.2016.

$\triangleright$ Claudius Zelenka and Reinhard Koch, "Single Image Plankton 3D Reconstruction from Extended Depth-of-Field Shadowgraph", in submission to the 3rd Workshop on Computer Vision for Analysis of Underwater Imagery (CVAUI 2018).

In this thesis algorithms based on a novel principle for the restoration of coherent images disturbed by diffractive effects are introduced. There are no prior algorithms capable of this task with this quality. This is the first essential contribution of this thesis.

The importance of this contribution can be seen by looking at a comparison of the capabilities of wavefront correction with existing algorithms and approaches. In Table 1.1 we see a comparison between phase retrieval, deconvolution algorithm, existing coherent image restoration and wavefront correction. It becomes clear that only wavefront correction (WFC) allows the high quality image restoration on coherent images and it also conducts phase retrieval as a side product. It reconstructs the phase information in the aperture plane of the restored image. More details on these related algorithms and approaches are presented in Chapter 2 and for details, on how WFC also conducts phase retrieval in the corrected wavefront, see Chapter 3. 
Table 1.1. Comparison of related works and WFC.

\begin{tabular}{|c|c|c|c|c|}
\hline & $\begin{array}{l}\text { Deconvolution } \\
\text { algorithms }\end{array}$ & $\begin{array}{l}\text { Phase retrieval } \\
\text { algorithms }\end{array}$ & $\begin{array}{l}\text { Related work } \\
\text { coherent } \\
\text { restoration }\end{array}$ & $\begin{array}{l}\text { Wavefront } \\
\text { correction } \\
\text { algorithm }\end{array}$ \\
\hline $\begin{array}{l}\text { Coherent } \\
\text { Images }\end{array}$ & No & Yes & Yes & Yes \\
\hline $\begin{array}{l}\text { Incoherent } \\
\text { Images }\end{array}$ & Yes & No & No & No \\
\hline $\begin{array}{l}\text { Phase is } \\
\text { retrieved }\end{array}$ & No & Yes & No & Yes \\
\hline $\begin{array}{l}\text { Image } \\
\text { restoration }\end{array}$ & Yes & No & Yes & Yes \\
\hline $\begin{array}{l}\text { Restoration } \\
\text { quality }\end{array}$ & High & No & Low & High \\
\hline
\end{tabular}

While phase retrieval is the inspiration for our wavefront correction algorithm, it is important to note that this thesis deals with a different task, namely the correction of wavefront distortions solely from a measured intensity distribution in the image plane. Additionally, some estimate of the wavefront distortion is needed. The task of image restoration can be accomplished neither with phase retrieval nor with the prior non-coherent image restoration algorithms (see Figure 4.25). Other coherent restoration algorithms, which are discussed in Chapter 2 have a low restoration quality (see Figure 4.8).

Furthermore, we use different constraints than prior phase retrieval algorithms, as we introduce an additional virtual focus plane, whose amplitude distribution we require to be real and positive.

The wavefront correction algorithm presented first, the WFC-GS (Wavefront correction-Gerchberg Saxton) algorithm is inspired by phase retrieval algorithms. We use this algorithm to introduce the underlying novel principles of wavefront correction. The restoration requires knowledge of wavefront distortion of the optical system and single image intensity distribution data from the image sensor. The WFC-GS algorithm iteratively applies the image plane and virtual focus plane conditions until convergence condition is satisfied.

In case of known aberrations, it uses only data, which are available 
1. Introduction

Table 1.2. Coherent image restoration algorithms used in this thesis.

\begin{tabular}{ccc}
\hline Method & Principle & Reference \\
\hline Coherent inverse filter & direct & [KDL13] [MKS+09] \\
\hline WFC-GS & projective & new \\
WFC-HIO & projective & new \\
WFC-AP & projective & new \\
WFC-RAAR & projective & new \\
WFC-HAAR & projective & new \\
\hline WFC-FISTA & proximal & new \\
\hline WFC-SS & projective, self-similarity & new
\end{tabular}

directly from of the image sensor. The algorithm can be easily parametrized with Zernike polynomials, which are very useful for the description of wavefront aberrations. For more details, see the Appendix A.5 with the orthogonal Zernike expansion.

Based on the WFC-GS algorithm and recent advances in mathematical optimization, several novel algorithm for coherent image restoration are presented. This is the second major contribution of this thesis. The improved algorithms achieve good results even on noisy real images and provide a large improvement over the basic algorithm.

These algorithms are based on projections onto convex sets: WFC-AP wavefront correction- average projection, WFC-RAAR wavefront correctionrelaxed average alternating reflections, WFC-HAAR wavefront correctionHaugazeau like average alternating reflections. Based on proximal optimization is WFC-FISTA, Wavefront correction - fast iterative shrinkage thresholding algorithm and the WFC-SS wavefront correction - selfsimilarity algorithm is based on the principle of fractal self-similarity. Table 1.2 gives an overview. We shall see that all Wavefront correction algorithms have unique properties. More details on related methods can be found in a dedicated chapter on related works, Chapter 2. 
We show in direct comparison how our novel algorithms are superior to related work algorithms and we present extended results and analysis in application on simulated images, real microscopic images of biological, medical samples as well as on images acquired with a shadowgraph. A further contribution is the development of a deep learning algorithm for the estimation of optimal focus for shadowgraph systems.

Furthermore, the application of the wavefront correction algorithms on images from a shadowgraph in oceanic expeditions is demonstrated, proofing that the algorithms are practically relevant. Another contribution of this thesis is the novel 3D and all-in-focus visualization of shadowgraph images.

In addition, we give an in-depth introduction and perspectives on the relation between wavefront correction, phase retrieval and non-coherent image deconvolution. We also give insights into the convergence and convexity of projective image restoration with wavefront correction and demonstrate that coherent imaging can be easily implemented even with a simple, low cost LED illumination. The results show that for coherent microscopy even very faulty imaging settings can be compensated with wavefront correction.

\subsection{Overview}

The following Chapter 2 focuses on related work in image deconvolution algorithms for non-coherent imaging and related work on the phase retrieval problem. These methods form the foundation for the wavefront correction algorithm, which is introduced with several variations in Chapter 3 . This chapter starts with a theoretical background and proceeds with introducing and discussing the core innovation of this thesis, the novel algorithms.

In the first section of Chapter 4, the results of the different variations of WFC on simulated images are presented and discussed. In the second section, results on microscopic images are explored. The third section introduces the laboratory shadowgraph measurement device and the implementation of wavefront correction on plankton images captured by it. This paves the way for further developments presented afterwards, such 


\section{Introduction}

as all-in-focus images and 3D visualization via depth from defocus using deep learning artificial neural networks trained to this effect.

Chapter 5 contains discussion of achieved results and a conclusion with vision and perspectives for future research and applications. The mathematical and physical backgrounds are discussed in the Appendix A, which covers relevant topics such as the scalar diffraction theory, projections onto convex sets and convolutional neural networks. The reader may choose to study the Appendix before continuing. It will be referenced in the following chapters to provide the reader with the necessary basic concepts. 


\section{Chapter 2}

\section{Related Work}

As explained in the previous section, the novel WFC algorithm is related to the idea of phase retrieval in a coherent imaging system. In spite of the shared features, it is important to note that WFC deals with a different task, namely the correction of wavefront distortions solely from a measured intensity distribution in the image plane. Additionally knowledge about the wavefront distortion of the optical system is needed. This task cannot be accomplished with phase retrieval or the prior non-coherent image restoration algorithms (see Section 4.2.7). An essential difference to the prior task of phase retrieval is that we use different constraint and introduce an additional virtual focus plane, where the amplitude distribution must be real.

In this chapter an overview over related work on image restoration and over related work on phase retrieval is given. Then some phase retrieval algorithm are explained in more detail, because of their relevance to WFC. Furthermore, we present related work on the focused shadowgraph.

\subsection{Coherent Restoration}

Coherent restoration techniques have growing importance, as can be seen by the very diverse areas of application contexts used in related work. In [KDL13] the aberrated image of a laser illuminated target is to be corrected to improve the image quality in coherence tomography. A correlation technique is used to estimate the phase error, which is corrected by Fourier space multiplication with the inverted estimated phase error.

The same restoration technique is used in [MKS+09] with prior phase error optimization based on a sharpness criterion. However both scale and 
application are entirely different, as the intention is to compensate the effects of atmospheric turbulence on an aerial image.

From the convolution theorem it follows, that a multiplication in Fourier space with a compensating phase is equivalent to a convolution with the Fourier transformed phase modulation or as it is called in this context filtering with an inverse PSF(point spread function), which is also known as inverse filtering [GL12] or spatial filtering. This technique of spatial filtering, while respecting Fresnel propagation, is the state of the art [CKC+06], which [SPD+09] explicitly confirms. It is also used in [LF15]. [SPD+09] also proposes a technique for the reconstruction of digital holographs using concept called cepstrum, however this approach has very strong conditions on the object-wave modulation and on the intensity of the reference wave.

In [GJV+13] spatial filtering is extended in a multi step approach to correct for the phase effects from off-axis tilt and curvature of the microscopic lens. Furthermore [SFM+11] gives good perspectives on the physical motivation and how this algorithmic spatial filtering can be replaced by a purely optical compensation for spherical phase aberrations. Another more recent application of this technique is used in [MS14] for $3 \mathrm{D}$ tracking of bacteria under coherent illumination. This work employs a strong denoising algorithm on the microscopic images, as the restoration algorithm seems to be very noise sensitive.

However, the most glaring weakness of all discussed related works on coherent image restoration is, that the phase of the aberrated image is ignored. This is not physically correct and ignores the fact that the phase of an image contains very important information as we show in Figure 2.1. Although discussed later in more detail we show that this is not an appropriate technique for high quality coherent image restorations, which is confirmed by results in Figure 4.8. This inherent problem is solved with the wavefront correction algorithm (WFC) introduced in this thesis which iteratively recovers both sharp image and phase.

Note that the method of Li et al. in [LMH+15] though also named wavefront correction, computes a correction of wavefront sensor measurements and does not restore measurements. 


\subsection{Image Deconvolution}

The image formation equation in the previous section is the foundation of all non-coherent restoration algorithms. The goal is to invert the influence of blur and recover a sharp image $I_{i n c}$ from Equation 1.1.1.

$$
O_{S I}=I_{i n c} \otimes B+N,
$$

where $O_{S I}$ denotes the captured input image, $B$ a blur kernel and $N$ additional noise. We need to distinguish between single image and multiple image approaches, which combine multiple frames for a better restoration. The most relevant works for this thesis deal with single images.

One can regard the problem of inverting the influence of this blur kernel as a deconvolution. Therefore, this subcategory of image restoration algorithms is known as deconvolution algorithms. All general incoherent image deconvolution methods rely on the blur model in equation 1.1.1.

As mentioned in the introduction whether prior knowledge of the blur kernel $B$ exists distinguishes between non-blind and blind deconvolution algorithm. If blindness is not explicitly stated, related works typically refer to non-blind deconvolution algorithms.

Historically one must distinguish between iterative and non-iterative methods. The most direct approach applies the inversion of the blur kernel on the image. This method has strict limitations for zero values in the blur kernel and high noise sensitivity. The most widely known non-iterative linear method is the Wiener filter [Wie13]. As early as 1931 iterative linear methods such as the van Cittert's method [Jäh02] were developed, a good overview is given in [Jan12] .

The Richardson-Lucy (RL) algorithm [Ric72], [Luc74] is the most common method in image restoration and can be seen as a standard algorithm in this field. RL-deconvolution is an iterative deconvolution algorithm, which is based on the statistical assumption that the observed image is the mean of a Poisson distribution. It maximizes the maximum likelihood (ML) of the restored image. For the RL-algorithm Fish et. al. [FWB+95] developed an adaption, which can be used if the PSF is unknown, expanding the scope of that algorithm to blind deconvolution. In this blind deconvolution algorithm in every iteration both the PSF estimation and the restored image are alternatingly refined. 
Maximum a posteriori (MAP) estimation is the result of Bayesian rules and allows finding the most likely sharp image explaining the observed distribution, given prior information about the probability distributions. Image priors are important to the success of modern blind and non-blind deconvolution algorithms such as [FSH+06] [KŠM13]. Much progress has been made in recent years in theoretically understanding how and why MAP applied to blind deconvolution is so successful and many algorithm were presented [LWD+09], [LWD+11] and [PF14].

Most algorithms incorporate a-priori knowledge about the image in terms of gradient sparsity, which is derived from natural image statistics [LFD+07]. Total variation is arguably the most common way to model this prior, see [CW98], [CEP+05] and more recent work in [Get12]. It is based on the assumption of locally smooth areas and it takes advantage of the $L_{1}$ norm. Different optimization strategies can be used for solving the resulting optimization problem, such as variable splitting, resulting in algorithms like FISTA [BT09a] [HRH+13].

Other methods use wavelet base priors [CCS+03], stochastic deconvolution [GHH+13] and Monte Carlo deconvolution [LYZ10].

All these priors are set from an external source, such as the mentioned natural image statistics. In [MI14] a different approach is used for deconvolution. Internal patch recurrence is based on the observation that in case of sharp natural images, structures or patches from an high resolution image, recurrently occur in low scale versions of the same image. In [MI14] internal patch recurrence is used as a prior for blind deconvolution.

Deconvolution using neural network has been studied for some years [Jan12] with limited success, until recently such methods have regained popularity due to the breakthroughs in machine learning with deep convolutional neural networks. Recent examples with competitive results are [XRL+14] [SBH+13].

In astronomy, restoration algorithms were developed almost simultaneously to the image processing community. Deconvolution from wavefront sensing (DWFS) is a term first defined by [PRF90] for the restoration of astronomic images disturbed by atmospheric turbulences with wavefront data from a wavefront sensor. From a simultaneously recorded intensity image $I_{i n c}$ and optical transfer function OTF (derived from wavefront $\phi$ ) the Fourier transform of restoration image intensity $I_{r}$ is calculated with 
[PRF90]:

$$
\mathcal{F}\left(I_{r}\right)=\frac{\mathcal{F}\left(I_{\text {inc }}\right) \mathcal{F}(\text { OTF })}{|\mathcal{F}(O T F)|^{2}} .
$$

Clearly, this approach does not consider the nature of coherent imaging. [Sch93] use multiframe techniques to either blind estimate the wavefront deformations or use them to refine a coarse estimate, which is called myopic algorithm [MRC+99]. Furthermore application specific priors are used such as the assumed Gaussian shape of the image from a star or a photon noise model [FMC+03] or Poisson noise [LSY+17] in intensity based MAP estimators. The same MAP approach with the incoherent imaging model is chosen in [MFC04] with myopic PSF estimation and an edge preserving prior.

$L_{1}$ total variation deconvolution with wavefront sensor data is applied in [RMZ12]. Miura et al. in [MOK+16] captures residual wavefront information with an additional wavefront sensor to further increase the image quality of deformable mirror compensated systems. From the wavefront measurements they calculate the PSF and apply deconvolution.

\subsection{Phase Retrieval}

Using the Fourier transform (see [Bra00][Kam00][Boa03] for a comprehensive introduction) any physical signal can be represented by its spectrum, using phase and amplitude. To demonstrate the information contained in the amplitude and phase, Figure 2.1 shows two different images (a),(b) and the same images with their phase exchanged (c) and (d). As visible from Figures 2.1(c) and (d) the phase dominates the image formation. The important content of the images is determined by the phase, therefore we can conclude that the phase includes more visible image information and is dominant for the visible impression.

In this section firstly an overview over the phase retrieval problem and algorithms solving it is given. Afterwards, the Gerchberg Saxton algorithm

\footnotetext{
${ }^{1}$ Author: Wikimedia I Simplon2010; Public domain: https: //commons.wikimedia.org/wiki/File\% 3Matterhorn_august_2009.JPG

${ }^{2}$ Author: Wikimedia I Dll; Public domain: https://commons.wikimedia.org/wiki/File\%3ACat public_domain_dedication_image_0011.jpg
} 
2. Related Work

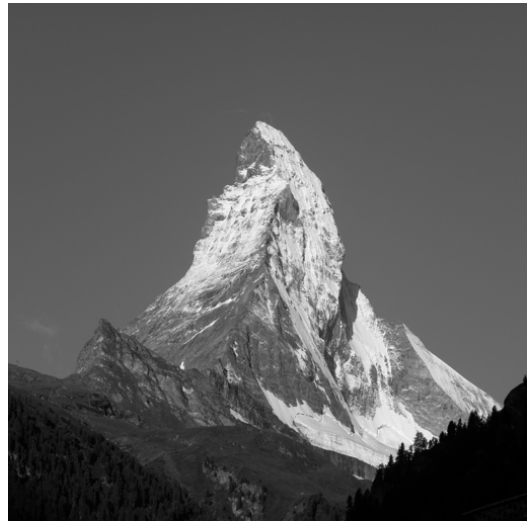

(a) Matterhorn ${ }^{1}$

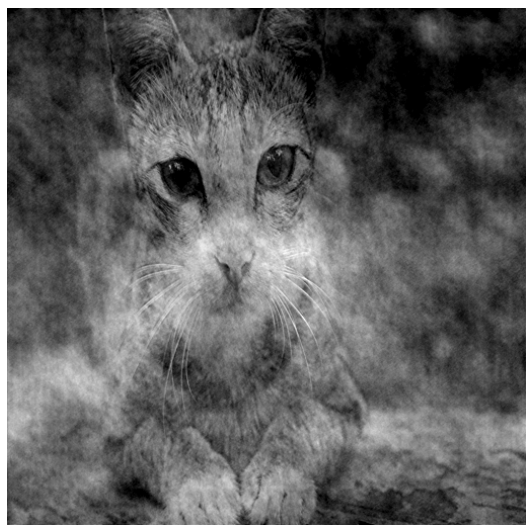

(c) Matterhorn image amplitude with cat image phase

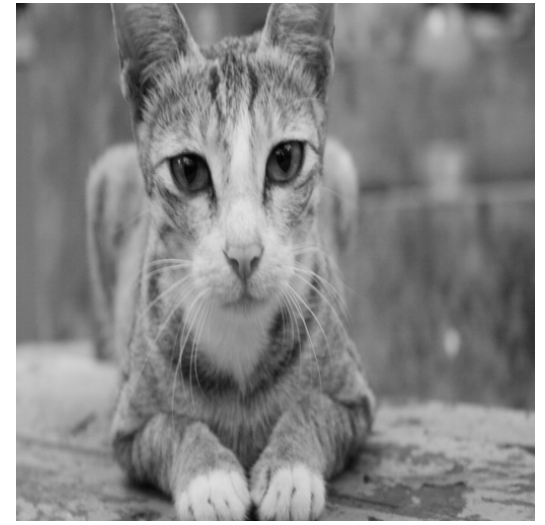

(b) $\mathrm{Cat}^{2}$

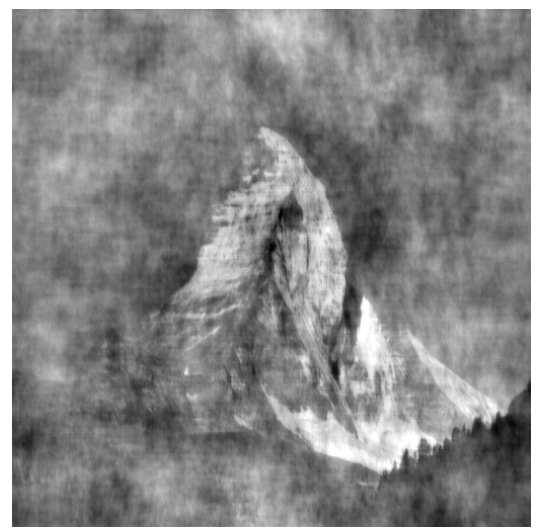

(d) Cat image amplitude with Matterhorn image phase

Figure 2.1. Two images are shown in (a) and (b), with their phase information exchanged in the Fourier-space in (c) and (d). Clearly, the phase holds most of the structural image information (inspired by [Osh12]). 


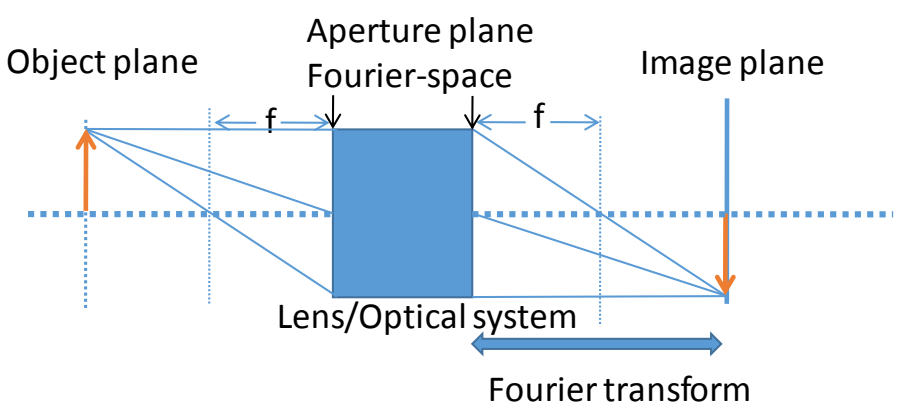

Figure 2.2. Optical system for phase retrieval with aperture plane $A$ and image plane $o . f$ denotes the focal length of the lens.

and its variation are explained in more detail, because it is the background for the wavefront correction algorithms introduced in this thesis.

Phase retrieval algorithms solve the task of restoring the unknown phase of a wavefront from measured intensity distributions in Fourierspace aperture plane and image plane with additional spatial constraints in the image plane as shown in Figure 2.2. The aperture plane can also be called pupil plane. This optical system is linear and generates no aberrations, real objects generate real images in the image plane. As a paraxial system, it can be represented with a single thin lens. The rays shown in the Figure 2.2 help visualizing the imaging process, but should be recognized as an inaccurate representation for a coherent system. Instead, the imaging process is based on the scalar diffraction theory introduced in the Appendix in Section A.1.

A more detailed overview of phase retrieval algorithms is given in [SEC+15], for a performance comparison of various algorithms see [SBP14]. In the area of coherent optics, phase retrieval algorithms are used in many applications from crystallography, holography to ptychography and astronomy. The classic phase retrieval algorithm is the GS algorithm [GS72]. It is discussed in the following section, together with the HybridInput-Output (HIO) algorithm for phase retrieval by Fienup [Fie82].

It can be shown that the Gerchberg-Saxton algorithm is equivalent to a steepest gradient descent algorithm (error reduction approach) [Fie82], 
and that it converges in the weak sense, which means that with each following step the RMS (root mean squared) error is either reduced or remains the same.

The original GS algorithm showed slow convergence especially in case of weak constraints in the image plane. Derived from the GS algorithm Fienup [Fie78] explored a series of variations, which are designed to deliver a faster convergence compared to the Gerchberg-Saxton algorithm. In the general implementation, there is one known set of constraints in the image plane and a second known set of constraints in the Fourier plane of an optical system. The task is to find matching phase distribution in both planes, which permits reconstruction in the image plane from Fourier plane measurements [Fie78]. Fienup's modified GS algorithms need less knowledge about the image plane and are thus widely used in the astronomy, where only weak constraints in the image plane are possible [Fie06]. Such constraints are typically the approximate size and non-negativity of the image [Fie87]. The Gerchberg-Saxton and Fienup algorithms can be seen as projective algorithms [BCL02] [Mar07a]. An overview of the applications of the Gerchberg-Saxton (GS) algorithm and its variations is given in [Fie13].

In general, phase retrieval is a very ill posed problem [JEH15]. Several works deal with the uniqueness of the phase retrieval problem. Uniqueness can be proven for the 1D case, the $2 \mathrm{D}$ or higher case is more difficult [SEC+15]. In recent results of [SS15] conditions for the exact reconstruction of a class of parametric 2D functions can be found.

A recent result is that for unique phase retrieval in finite $M$ dimensional vector spaces $4 M-4$ measurement vectors are necessary. This is presented and proven for the two and three dimensional case in [BCM+13]. Notable recent developments in algorithms for efficient phase retrieval are the alternating minimization approach by [NJS15] and the application of a optimization technique called 'Wirtinger flow' [BSZ+15]. For the scope of this thesis only single observation methods are important, in other works this distinction is not made as clearly. Many recent works, such as [CLS15] treat the multiple observations with different phase conditions as the standard case, testing them on a single observation fails. The reason for this development lies in modern high-speed sensors making it easy to acquire multiple measurements [JEH15]. Many modern phase retrieval 
algorithm leverage the high acquisition rates of modern sensors and use multiple measurements, as the base of the algorithm. If more than one intensity measurement is available, the SBMIR (Single-Beam-MultipleIntensity-Reconstruction) algorithm [POZ05] [BW01] can be used for phase recovery. SBMIR requires multiples intensity measurement in planes at known distances from the aperture. It starts in the first plane from the aperture with an arbitrary phase, and iterates through all available planes ordered by their distance, by calculating the light propagation of the angular spectrum from one plane to the next and replacing the calculated intensity with the measured intensity, see also [FKJ+12]. After the last plane, it closes the loop by going back to the first plane. An extension with multi resolution techniques and relaxations parameters is suggested in [FKK+14].

Another current research direction is exploiting sparsity. While the modern sensors mentioned above allow a high speed acquisition, they also have low spatial resolution. Examples of such works are GESPAR [SBE14] [FWW+14] or oversampling in PHASELIFT [CSV13].

A recent development is quantum phase retrieval [LIP+16] based on [OP94] which applies phase retrieval to images obtained by illumination with quantum states.

In the following some of these phase retrieval methods will be explained in more detail. We start with the Gerchberg-Saxton algorithm, as it was the first phase retrieval algorithm, then the Fienup Hybrid-inputoutput algorithm and other selected projection based methods, because they are relevant to the WFC-algorithm and the analysis in Chapter 3.

\section{The Gerchberg-Saxton Algorithm}

The first phase retrieval algorithm was presented by Gerchberg and Saxton in [GS72] , the Gerchberg-Saxton (GS) algorithm. Because of its relevance to the following Wavefront Correction algorithm, it is explained in more detail.

The imaging system shown in Figure 2.2 is composed of an incident light, a lens with aperture plane $A$ and an image plane $O$. While an overview of the scalar diffraction is given in the Appendix A.1, this is the basic imaging system used to explain and discuss this algorithm and as 
such, it requires further explanation. In a coherent system, the aperture plane $A$ of a thin lens will be called Fourier plane of the thin lens, while the image is placed in the focus. It is called Fourier plane, because this coherent optical system is based on diffractive optics and hence the image planes light distribution can be derived from the aperture plane light distribution via the Fourier transform. The inverse holds also true, the inverse Fourier transform of the aperture plane yields the light distribution in the image plane. This convention of using the positive Fourier transform to convert from object space to Fourier-space and the inverse Fourier transform to transform back will be used in this thesis, it is the standard convention in phase retrieval algorithms [GS72].

The vector space of image plane images is called 'object-space' and the vector space of the aperture plane is called Fourier-space. The light distributions in either plane can be described by its spatial frequency phase and amplitude. Both can be expressed as a function of complex values. The complex field in the image plane $O$ is composed of amplitude $O_{a}$ and phase $\phi$ by

$$
O(x, y)=O_{a}(x, y) e^{j \phi x, y} .
$$

Given such a system, the Gerchberg-Saxton (GS) Algorithm is capable of retrieving the phase of the both planes, given intensity measurements in both planes only. The algorithm needs amplitude distributions in the image plane and in the Fourier-space aperture plane, which can be derived as square root from the measured intensity distributions. The amplitude distribution in the image plane is $O_{0}(n)$ and $A_{0}(k)$ in the Fourier plane. We use $n$ as the spatial parameter of the image plane and $k$ as the spatial parameter in Fourier space. For clarity and conformity with related works [GS72] we denote the algorithm for the one-dimensional case without loss of generality.

The amplitude distributions across the image plane $O$ and a Fourierspace plane $A$ are linked by the Fourier transform operator $\mathcal{F}$ :

$$
O(n)=\mathcal{F}(A)(n)=\int A(k) \exp (-j 2 \pi k n) \mathrm{d} k .
$$

The algorithm starts with an arbitrary phase distribution in $O$ and loops over object-space and Fourier-space, while enforcing the constraints 
in every iteration $i$, which is noted as a subindex, (see Figure 2.3):

$$
\begin{aligned}
A_{i}(k) & =\mathcal{F}\left(O_{i}\right)(k) \\
A_{i}^{\prime}(k) & =\left|A_{0}(k)\right| \frac{A_{i}(k)}{\left|A_{i}(k)\right|} \\
O_{i}^{\prime}(n) & =\mathcal{F}^{-1}\left(A_{i}^{\prime}\right)(n) \\
O_{i+1}(n) & =\left|O_{0}\right| \frac{O_{i}^{\prime}(n)}{\left|O_{i}^{\prime}(n)\right|} .
\end{aligned}
$$

A disadvantage of this basic algorithm is its slow convergence. This was greatly improved by Fienup in [Fie82]. As already mentioned, the algorithms have been designed for situations, where the constraint in the image plane is only the approximate image size, which is called support. The Hybrid-Input-Output (HIO) algorithm is widely used today.

This algorithm treats the application of Fourier domain constraints as a feedback system with the input $O_{i}$ in Equation 2.3.3 and output $O_{i}^{\prime}(n)$ in Equation 2.3.5. Furthermore, an additional support constraint is added, which restricts the size of the reconstruction to a prior defined set of points. The object-space update Equation 2.3.6 is changed to:

$$
O_{i+1}(n)= \begin{cases}O_{i}^{\prime}(n) & n \in V_{o} \\ O_{i}(n)-\beta O_{i}^{\prime}(n) & n \notin V_{o},\end{cases}
$$

where $V_{o}$ is a set of points, where the object-space constraints are valid and $\beta \in \mathrm{R}$ is a parameter influencing the convergence speed. A value commonly used is 0.7 , which was determined experimentally as optimal in most cases [Fie13], we follow this convention. For an evaluation of the influence of this parameter see [Fie82].

Fienup [Fie87] has shown, that the magnitude constraint in object space can be relaxed, as the knowledge of approximate object size is sufficient. Nevertheless, the algorithms of Fienup and similar still require the magnitude constraint in the aperture plane, which means having knowledge about it and are therefore not applicable for the restoration of blurred images, which is the focus of this thesis.

From an input image, that is violating a constraint, the enforcement of a constraint outputs a result, which is the first valid input closest to that 


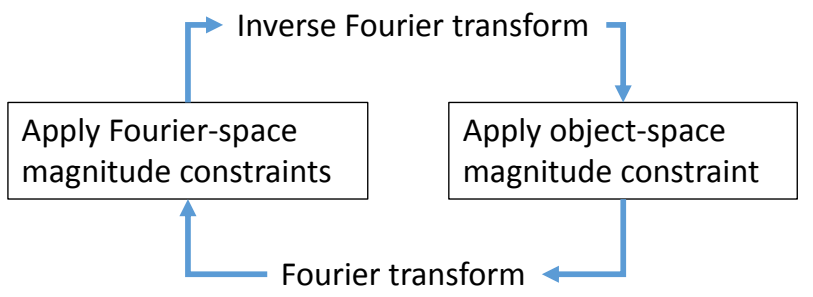

Figure 2.3. The Gerchberg-Saxton algorithm.

violating input. This application of a constraint can also be expressed in the framework of projections [YW82], where the application of a projection means the enforcement of a constraint. Let $V$ be the set of values for which a constraint holds, then we define a projection $P$ of $y$ onto $V$ as the set of values $x \in V$, which are closest to $y$ :

$$
P_{V}(y)=\{x \in V,\|x-y\|=\|y-V\|\} .
$$

For practical purposes if the set has more than one value, we choose one of these points. For conditions for single-valuedness of the Fienup constraints and a more detailed introduction see [BCL02]. The application of the constraint in Equation 2.3.6 is defined as $P_{O}$ and the subsequent application of Equations 2.3.3, 2.3.4 and 2.3.5 as $P_{A}$. The HIO algorithm now reads:

$$
O_{i+1}(n)= \begin{cases}P_{A}\left(O_{i}\right)(n) & n \in V_{o} \\ O_{i}(n)-\beta P_{A}\left(O_{i}\right)(n) & n \notin V_{o} .\end{cases}
$$

It was discovered later [LS83] [YW82] [BCL02] that the GerchbergSaxton algorithm can also be seen in the context algorithm of projections onto convex sets (POCS), in which every application of a constraint is a projection onto a set of valid points. Thus, the GS algorithm can be expressed as

$$
O_{i+1}(n)=P_{o}\left(P_{A}\left(O_{i}(n)\right)\right) \text {. }
$$

This makes the algorithm structure very clear, which is alternatingly projecting onto each set until convergence. This alternating application of projections in the Gerchberg-Saxton algorithm has previously been established as the von-Neumann algorithm [Neu51] and is also called the 
$\mathrm{MAP}($ multiple alternating projections) algorithm [ER11] in the field of general projection algorithms for solving the problem of finding intersecting points in convex sets.

In this section we established the connection from phase retrieval methods to POCS algorithms and as [BCL02] shows using general POCS algorithms for phase retrieval is also possible. An introduction into POCS algorithms is given in Section A.6. Most of the algorithms have been applied and evaluated with success to phase retrieval [Mar07a]. An exception are the Haugeauzou algorithms, which are the only algorithms which are strongly convergent on convex sets [BCL06]. It is important to note that while the convergence of these methods can be proven, this result is applicable only under the assumption that the underlying sets are convex. However, this is not the case for the phase retrieval problem, for a proof see [Mar07a].

\subsection{The Focused Shadowgraph}

In this section first we introduce the shadowgraph and give the reader a basic understanding of its optical elements and properties. Furthermore, we discuss related works applying a shadowgraphs or similar optical devices for research into marine plankton.

The focused shadowgraph is a imaging concept meant for the observation of small in-transparent or semitransparent objects [Set01]. A modern introduction into shadowgraph systems can be found in [Maz13].

A typical focused shadowgraph is composed of the following elements. Light of a point light source is converted with a condensation lens into parallel light. This light is used to illuminate the target of observation at which point the opaqueness of the target creates an image of light and shadow. With a second condensation lens this light is focused into the focusing lens. The focusing lens has the purpose of selecting a plane of focus. It projects the light and shadow image from the target onto the image sensor. The basic shadowgraph theory is based on geometrical optics. In this thesis the shadowgraph is converted to a coherent optical instrument with a standard LED as illumination.

An optical drawing of a focused shadowgraph principle is shown in 


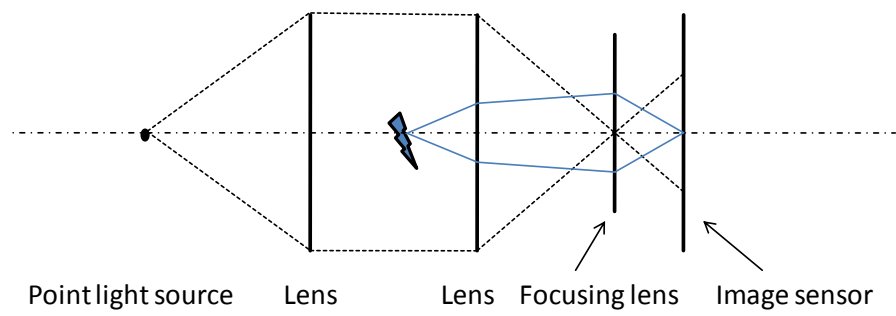

Figure 2.4. Optical drawing of the focused shadowgraph. Black dots show the illumination edge rays, blue the rays from the objects projection onto the camera sensor. Shown is an adaptation of a drawing from [Set01].

2.4. With the parallel light source, the observation objects are projected onto the image sensor and become visible as shadows.

The shadowgraph itself has numerous applications. It can be used for particle image velocimetry (PIV) [WYH08] in which the motion of particles inside a volume is observed and turbidity measurement in to acquire images of semi-transparent objects. An overview of modern shadowgraph imaging for the analysis of fluid dynamics in jets and drops is given in $[C C M+11]$. Shadowgraphs are also used for dust particle measurements in high sensible environment such as the ITER (International Thermonuclear Experimental Reactor) [GMC+15]. Although employed since the 17th century [Set01], research on the shadowgraph is ongoing, as recent works on smartphone based shadowgraph systems show [Set17].

Another field of application is marina plankton observation and measurement. This is the field in which, due to the cooperation between the Workgroup on Multimedia Information Processsing of Prof.Dr-Ing. Reinhard Koch at Kiel University and GEOMAR Helmholtz Center for Ocean Research Kiel, we are particularly interested in and on which we will focus in this section.

Plankton observation and measurement is of very high importance foremost for marine biology [HWL+00], because plankton has impact on the entire marine habitat. Moreover plankton is also an indicator of other phenomena such as climate change [HRR05] [Ric08] and marine 
acidification [OFA+05][HDÁ10]. Because of this global interrelationship, the results can influence and have impact on many other fields of research such as climate modeling.

A focused shadowgraph system for plankton observation 'In situ ichthyoplankton imaging system' ISIIS is presented in [CG08]. It uses a collimated pin hole led illumination inside a stainless steel tube housing and a camera in a second tube housing. Both tubes are axially arranged and plankton is observed by projecting light from the illumination tube towards the camera tube. The system achieves a resolution of $68 \mu$, which seems to be sufficient for most plankton objects.

The ISIIS and its novel version the ISIISv2 plankton imagers are a product of Bellamare, LLC of San Diego, California ${ }^{3}$. In addition to plankton shadowgraph systems, the company offers in-house analysis and image sorting services for the acquired images. An application of this system in a multimodal survey including plankton nets and ultrasound in the southern california bight can be seen in $[\mathrm{MCN}+12]$, where the results are used to produce high resolution plankton distribution maps of the area. These maps are of importance to marine biologists, as they help to understand how other environmental features correlate with plankton distribution.

Research towards low-cost autonomous plankton observation platforms like the 'GUARD1' system [MCM+15] [CMM+16], which sample the ocean in a swarm and therefore provide more data is ongoing. Another recent work on semi-automated underwater microscopy is [GNM+16], which uses automated image acquisition, processing and analysis device for the observation of fish larvae.

From the various sources dealing with plankton observation we follow, that the shadowgraph is the superior system for in situ observation [CGG+13] [GCG+15]. The shadowgraph is also used in laboratory environments to get more in depth knowledge on plankton behavior. In [YRW08] orthogonally overlapping shadowgraphs allow the reconstruction of copepod (a group common plankton species) [YLG+92] trajectories.

\footnotetext{
${ }^{3}$ http:/ / www.planktonimaging.com/
} 
In any expedition thousands of images are collected, which makes manual evaluation unpractical and calls for an automatic analysis. Moreover, for autonomous or semi-autonomous acquisition systems such an automatic analysis of the images is required. The automatic analysis of the plankton images still remains a challenge. In 2015 the machine learning contest organizer Kaggle issued the machine learning contest 'National Data Science Bowl' with data by the Hatfield Marine Science Center at Oregon State University. In this contest shadowgraph plankton images were to be classified by the plankton species. The competition featured $175000 \$$ in prize money and was well received with entries from 1049 teams [CSR+15] [XWC+15].

In [FPL+16] an imperfect classification of ISIIS shadowgraph plankton images is used to describe distribution patterns of different species on the Ligurian coast. A complete shadowgraph plankton observation system is presented in [BGB+15], in which a complete pipeline including background subtraction, noise removal, segmentation and image analysis for shadowgraph plankton images is presented. Because $[B G B+15]$ is published under a special free license, we are able to show original images from this publication and also show images from [BGB+15] enhanced with the novel algorithms of this thesis in Chapter 4.

As explained in Section 4.4.2 in this thesis a method for the extraction of three dimensional data from single shadowgraph plankton images is developed. Related work for three dimensional in-situ plankton observation is scarce. One such work is [LYI+13] which presents the Japanese plankton imaging system 'uROW PICCASSo', which uses dark field illumination with ring led allowing full color images of plankton and also mentions experiments with light field camera for three dimensional imaging, however no results are shared. 
Chapter 3

\section{The Wavefront Correction Algorithm}

\subsection{Description of the Algorithm}

This chapter begins with a motivation in optical terms, which leads to the formulation of the WFC-GS algorithm. Building on this other formulation of the Wavefront correction problem and more sophisticated algorithm and approaches from other fields are included, to find the optimal solution for the WFC problem. Furthermore, the theoretical convergence properties of the novel WFC algorithms under the different conditions are discussed.

In a coherent system, the light distribution in the focus plane of a thin lens is the Fourier transform of the light distribution of the aperture (Fourier plane). Thus assuming that the input image is captured in perfect focus and assuming there are no aberrations we can switch between this focal image plane and the Fourier plane by applying the Fourier transform and the inverse Fourier transform. As explained in the introduction of classic phase retrieval algorithms in Section 2.3, we follow the standard convention of using the positive Fourier transform to convert light distributions from image plane to aperture plane.

However, if the image is not captured in perfect focus or if any other aberrations occur, we need to consider the additional wavefront deformation introduced by defocus or other optical aberrations.

To this effect, the wavefront deformation has to be superposed to the original wave. Aberrations are described as wavefront deformations, which can be described as the optical path difference across the aperture. As will be shown, the defocus is represented by a superposition of a spherical phase term. If there are no aberrations, then the optical path difference 
and wavefront deformation is zero and the image in the image plane is undistorted.

In a real environment the optical system is never ideal, the image is blurred. The blur is caused by imperfections of the whole optical imaging system and can be expressed as wavefront deformation. The task of image restoration deals with methods for the correction of the wavefront deformation.

This thesis deals with the imaging configuration shown in Figure 3.1. It is an optical imaging system with object plane, aperture plane, focus plane and image plane. The optical system consists of a lens, which may in the ideal aberration-free case be represented by a thin lens. The lens forms a focused image of the object in the focus plane. In Figure 2.2 (the optical system for phase retrieval) the image plane is identical with the focus plane of the lens, while in Figure 3.1 the image plane is shifted from the sharp focus plane, which is equivalent to the superposition of a spherical wavefront deformation.

This spherical wavefront deformation can be visualized by points across the aperture plane of equal phase of the light wave field. It is shown on the left side of Figure 3.1 with the aperture plane in normed x-y coordinates and the optical path difference as a colored connected surface.

As highlighted previously, the rays are meant as a help for visualizing the imaging process and are inaccurate for a coherent system, in which the light propagation is ruled by diffraction. The imaging process is based on the scalar diffraction theory introduced in the Appendix in Section A.1.

The wavefront aberration is usually approximated by Zernike polynomials [Nol76] in modern optics. In this thesis the Zernike polynomials are used for the simulation of wavefront aberration, therefore they will be described in some detail. Zernike polynomials describe the deformation in radial coordinates, as distance from the center of a unit disc and angle. Furthermore, they form the basis of a vector space, which makes them ideally suited for the description of wavefront aberrations. This means any aberration can be described as a Zernike polynomial or a combination of Zernike polynomials. An introduction to Zernike polynomials is given in Section A.5 of the Appendix.

A wavefront deformation $W$ is described as the sum of Zernike poly- 
nomials $Z_{i}$, with parameters $p_{i}$ :

$$
W=\sum_{i} Z_{i} p_{i}
$$

In the following the function of a lens is discussed shortly. For coherent light incident on a convex thin lens with radius $r$, where the paraxial approximation is valid, the spherical phase delay is

$$
\phi_{d}(x, y)=\frac{k}{2 r}\left(x^{2}+y^{2}\right)
$$

or written in multiplicative form:

$$
\phi_{m}(x, y)=\exp \left(j \frac{k}{2 r}\left(x^{2}+y^{2}\right)\right),
$$

where $k=\frac{2 \pi}{\lambda}$ is the wave number and $x$ horizontal and $y$ vertical distance from the center of the lens perpendicular to the optical axis. For a derivation and more explanations, see appendix Section A.1.4.

The spherical wavefront deformation due to wavefront propagation can be described as

$$
\phi_{p}(x, y)=-\frac{\pi}{\lambda z}\left(x^{2}+y^{2}\right),
$$

where $\lambda$ denotes the wavelength and $z$ the distance of the image from the exit pupil. A derivation of this equation can be found in Section A.1.3). As can be seen, the addition of an appropriate spherical phase can be used to simulate or compensate the defocus.

The optical wave, which propagates from the aperture plane, has a diverging spherical wavefront as discussed in the Section A.1.3. The focusing lens changes the diverging wavefront to converging and creates a sharp image with planar phase.

If the image plane is moved from the focus, as shown in Figure 3.1, the phase of the image is defocused and no longer planar. Notice that in this image the term focus plane is assigned to the sharp image. This convention will be used often in the following chapters. The image plane is assigned to the measured and blurred image.

Changing the radius of the lens has the same effect as moving the image plane away from the focus plane. In both cases, the wavefront in the image plane is changed by an additional spherical component. In the focus plane the inherent divergence of the light is compensated by the focusing 


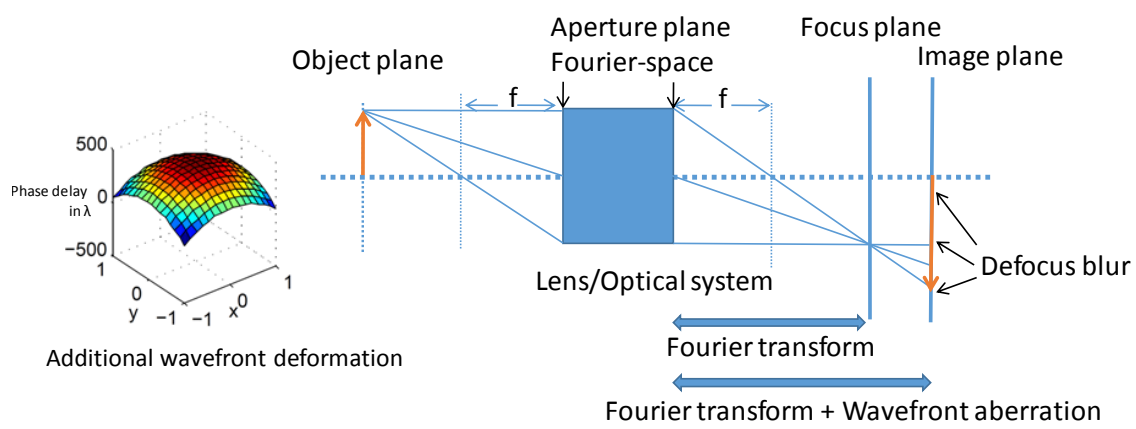

Figure 3.1. Wavefront correction in an out of focus optical system. Compared to Figure 2.2, the image plane is out of focus, which causes an additional spherical wavefront deformation. The wavefront deformation is visualized as a surface of points with equal phase across the $x-y$ coordinates of the aperture plane.

optical system and thus the image has planar phase. This is discussed with more rigor in the appendix in Section A.2.

Let us consider an image distorted by wavefront aberrations. If we had complete information about the light distribution in the Fourier plane/aperture plane of the imaging system in phase and amplitude, adding the compensating wavefront and applying the inverse Fourier transform is all we needed to recover a sharp image. This is the approach taken in related work on coherent image restoration, see Section 2.1, without having this phase information.

However, practical image restoration is not that simple, because in most applications, such as in a typical microscopic setting, we have no sensors to measure the Fourier space magnitude and phase. Therefore and because of the disturbed image in the imaging plane, phase retrieval algorithms are not applicable. The goal is to develop an image restoration algorithm, based solely on the knowledge of the intensity distribution of the distorted image and some measure of the wavefront deformation.

Therefore, we can use two constraints: The first constraint is the measured amplitude distribution, which is the square root of the intensity distribution, the second one is the requirement for a real and positive am- 
plitude distribution in the virtual ideal focus plane, where all aberrations are removed.

We enforce a zero angle phase vector by calculating the magnitude, effectively setting the amplitude to real and positive values. Note that in a restored image, positive or negative amplitude values would show the same intensity. The application of phase aberration function $\phi_{s}$ or its inverse $\phi_{s}^{-1}$ means a multiplicative phase shift on the corresponding complex amplitudes. It is defined analogous to the specific defocus phase shift $\phi_{m}$ in Equation 3.1.3.

\subsubsection{Physical Introduction and WFC-GS}

To reach an algorithmic description of the algorithm we start with the virtual ideal focus plane. It is introduced as virtual focus plane $F$, which can be reached from Fourier-space $A$ via the inverse Fourier transform $\mathcal{F}^{-1}$. The following equations define the Gerchberg-Saxton (GS) inspired version of the wavefront correction algorithm (WFC-GS) in iteration step $i$, illustrated by Figure 3.2:

$$
\begin{aligned}
A_{i}(k) & =\mathcal{F}\left(O_{i}\right) \phi_{s}^{-1}(k) \\
F_{i}(n) & =\mathcal{F}^{-1}\left(A_{i}\right)(n) \\
F_{i}^{\prime}(n) & =\left|F_{i}(n)\right| \\
A_{i}^{\prime}(k) & =\mathcal{F}\left(F_{i}^{\prime}\right)(k) \\
O_{i}(n) & =\mathcal{F}^{-1}\left(A_{i}^{\prime} \phi_{s}\right)(n) \\
O_{i+1}(n) & =\left|O_{0}(n)\right| \frac{O_{i}(n)}{\left|O_{i}(n)\right|} .
\end{aligned}
$$

The algorithm starts similar to the GS-algorithm with the measured $O_{0}$ as $O_{i}$. In the first step (Equation 3.1.5) the Fourier transform is applied. Then the inverse wavefront deformation is applied by multiplying the Fourier space distribution with inverted phase delay $\phi_{s}^{-1}$ of this wavefront. Transferred to the virtual focus plane (3.1.6), the real and positive constraint of the distribution is enforced (3.1.7). Then we transform back into objectspace $(3.1 .8,3.1 .9)$, enforce the object magnitude constraint (3.1.10) and 


\section{The Wavefront Correction Algorithm}

start iteration $i+1$.

The algorithm starts in object space with the measured magnitude distribution.

A sequence of $O_{i}$ gives the image plane amplitude distribution in iteration step $i$. In the last step of the algorithm the object magnitude is set to the initial magnitude with application of Equation 3.1.10. Thus $O_{i}$ is in fact the complex combination of the initial magnitude from the square root of the measured intensity $\left|O_{0}(n)\right|$ and phase information gained during the course of the algorithm. Therefore, we can conclude that wavefront correction algorithm also conducts phase retrieval as it is noted in Figure 1.1.

However, these values of the sequence of $O_{i}$ are not the practical result of the algorithm, as just explained they denote the object space distribution. The aim of wavefront correction is to restore the sharp image, which resides in the focus plane. Hence, after the convergence criterion is reached the final iterative $O_{i}$ of the object space must be transferred to focal space. This is achieved by applying the Fourier transform to reach Fourier space, then the wavefront deformation and finally an inverse Fourier transform into focal space. These steps are equivalent to applying Equation 3.1.5 and Equation 3.1.6. Convergence criteria are discussed in more detail in Section 3.6.

For the practical application of the algorithm, note that although the exact phase magnitude $k /(2 r)$ of Equation 3.1.3 depends on the wavelength, exact knowledge is not necessary, because it is only a linear factor that can be compensated with the radius. More practical is a measure of maximum phase difference dependent on the image size.

\subsection{Projection Algorithms}

In the previous section the WFC-GS algorithm was introduced within the domain and notation of optical imaging. In this section an alternative view is sought. Similar to phase retrieval for the wavefront correction (WFC)algorithm the fulfilling of constraints can be described in the framework of projections onto convex sets. The question of actual convexity of WFC is discussed later. 


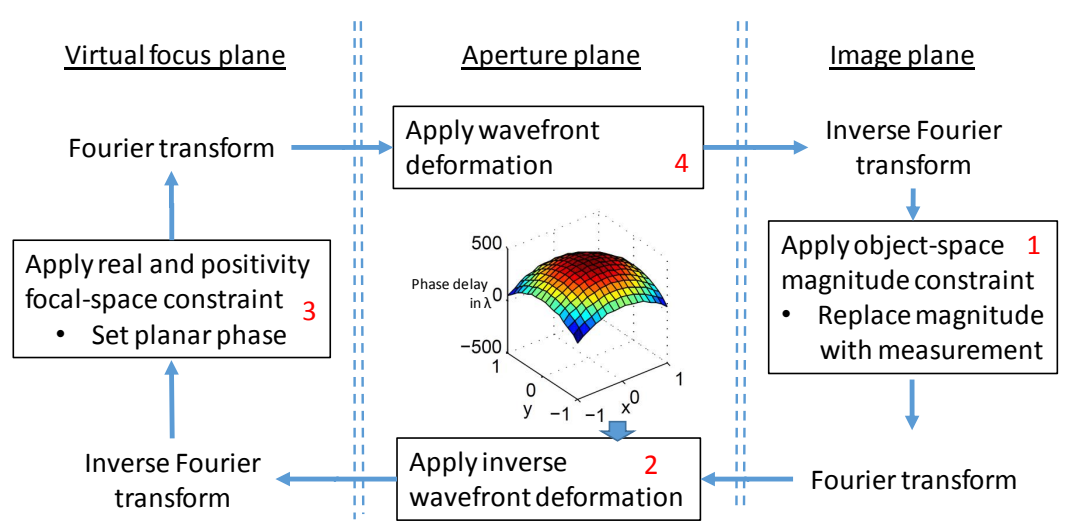

Figure 3.2. Overview of the WFC-GS algorithm.

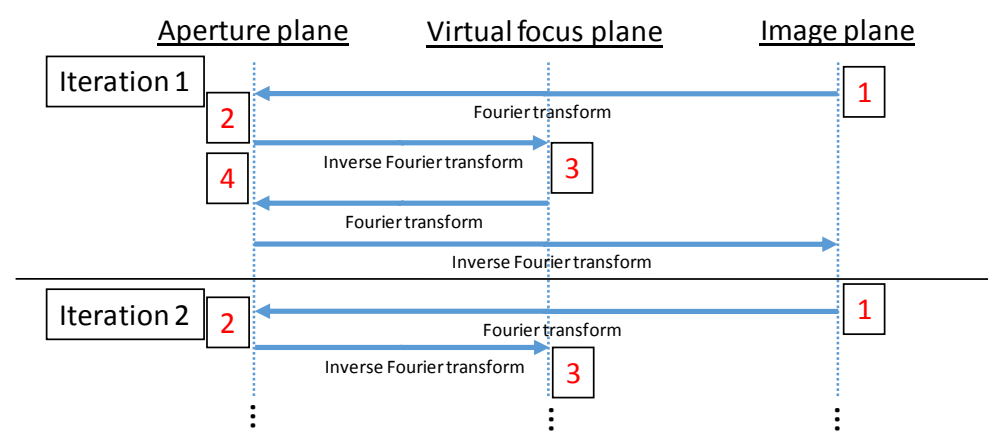

Figure 3.3. WFC-GS algorithm iterations. The planes are arranged as in Figure 3.1. The numbers resemble the same steps as in Figure 3.2. 
In the most general sense, the enforcement of a constraint projects an element from the sets of possible data points onto the set of valid data points, i.e. points which do not violate the constraint. In the general non-convex case, where multi-valuedness of the projectors is an issue, this can be mostly resolved by arbitrarily choosing a valid data point if the projection result is multivalued [BCL02].

With the projective formulation of wavefront correction in terms of the POCS (projections onto convex sets), the Gerchberg-Saxton algorithm can now be identified as the von-Neumann algorithm [Neu51].

The WFC-GS algorithm includes two constraints, the focal space constraint and the object space constraint. We denote the application of the object space constraint of Equation 3.1.10 as projection $P_{o}$ and the application of virtual focal space constraint including necessary transformations in Equations 3.1.5 to 3.1.9 as projection $P_{F}$.

The WFC-GS algorithm can now be written as

$$
O_{i+1}(n)=P_{o}\left(P_{F}\left(O_{i}(n)\right)\right) .
$$

This algorithm uses the idea of the Gerchberg-Saxton phase retrieval algorithm in order to iteratively optimize the image. The Fienup variations of the Gerchberg-Saxton algorithm are introduced in Section 2.3 and offer advantages over the original Gerchberg-Saxton algorithm such as a faster convergence. The most notable algorithm by Fienup is the Hybrid-InputOutput (HIO) algorithm.

Because we lack information about the object support, we cannot directly apply the standard HIO definition in Equation 2.3.9. Therefore, adapting wavefront correction to use the principle of the HIO algorithm requires some more changes than including focus-space constraints . Let $\mathbf{1}_{V}$ be the characteristic function of the set of points $V$ where the constraints (such as object support) are valid and $\mathbf{1}_{C V}$ of its complement. With [BCL02] we write the HIO-version of the wavefront correction algorithm (WFC$\mathrm{HIO})$ as:

$$
\begin{aligned}
O_{i+1}(n) & =\mathbf{1}_{V} P_{F}\left(O_{i}\right)(n)+\mathbf{1}_{C V}\left(O_{i}-\beta P_{F}\left(O_{i}\right)\right)(n) \\
& =\mathbf{1}_{V} P_{F}\left(O_{i}\right)(n)+\left(\mathbf{1}-\mathbf{1}_{V}\left(O_{i}-\beta P_{F}\left(O_{i}\right)\right)(n)\right. \\
& =\mathbf{1}_{V}\left((1+\beta) P_{F}\left(O_{i}\right)-O_{i}\right)+\left(I-P_{F}\right)\left(O_{i}\right)(n)
\end{aligned}
$$




$$
=\left(P_{o}\left((1+\beta) P_{F}-I\right)+I-P_{F}\right)\left(O_{i}\right)(n)
$$

and can apply it on images with wavefront aberration, without requiring object support information.

Both of these algorithms have the disadvantage of being only weakly convergent, which we showed for WFC-GS in Section 3.3 and [ZK16]. This projective formulation allows us to use more advanced POCS algorithms introduced in the Appendix A.6 to derive novel wavefront correction algorithms. To express the constraint applications as projections, we always identify one of the projections as the image plane constraint projection $P_{o}$ and the other as the focus plane constraint projection $P_{F}$.

We apply the averaged projection (AP) algorithm [ER11] to wavefront correction. This leads to the WFC-AP algorithm with update formula

$$
O_{i+1}(n)=\frac{1}{2}\left(P_{o}\left(O_{i}(n)\right)+P_{F}\left(O_{i}(n)\right)\right),
$$

where both constraints are applied and the results is averaged.

The RAAR (Relaxed averaged alternating reflections) algorithm by Luke [Luk05] is a POCS algorithm with improved convergence. The WFCRAAR algorithm is defined by this update formula:

$$
O_{i+1}(n)=\left(\frac{1}{2} \beta\left(R_{o} R_{f}+I\right)+(1+\beta) P_{F}\right) O_{i}(n),
$$

where $R_{o}$, and $R_{f}$ denote reflectors and $\beta$ a dampening parameter between 0 and 1 .

A recent contribution by Bauschke et al. is a strongly convergent POCS algorithm based on concepts by Haugazeau [Hau68], the HAAR algorithm. The significance of this algorithm lies in its strong convergence properties, which are discussed the Appendix.

As a Haugazeau based algorithm, the WFC-HAAR algorithm reads

$$
O_{i+1}(n)=Q\left(O_{0}(n), O_{i}(n),\left(1-\mu_{n}\right) O_{i}(n)+\mu_{n} T O_{i}(n)\right),
$$

with $\left(\mu_{n}\right)_{n \in \mathrm{N}}$ an arbitrary sequence of values in $\left.] 0,1\right]$ with $\inf _{n \in \mathrm{N}} \mu_{n}>0$, and $Q$ the Haugazeau helper function (Appendix, Equation A.6.11). 
3. The Wavefront Correction Algorithm

\subsection{Convergence}

The question of convergence is of both theoretical and practical importance as it defines how usable the algorithm can be. In this section the convergence of the methods introduced before is discussed.

In [Fie82] the weak convergence for the GS algorithm is shown using an error reduction argument, we expand on this to prove the same property for the GS-version of the wavefront correction algorithm (WFC-GS).

In iteration $i$ the squared error against the object-space image plane constraint over the entire image is

$$
E_{O, i}^{2}=\int_{n}\left(O_{i+1}(n)-O_{i}^{\prime}(n)\right)^{2} \mathrm{~d} n .
$$

Similar, for the virtual focus plane constraint the squared error is

$$
E_{F, i}^{2}=\int_{n}\left(F_{i}(n)-F_{i}^{\prime}(n)\right)^{2} \mathrm{~d} n .
$$

The squared error may also be seen as the energy of the error. With Parseval's theorem it follows that

$$
E_{F, i}^{2}=\int_{k}\left(A_{i}(k) \phi_{s}^{-1}-A_{i}^{\prime}(k) \phi_{s}^{-1}\right)^{2} \mathrm{~d} k
$$

Because of the energy conservation of optical light transport, we obtain

$$
E_{F, i}^{2}=\int_{k}\left(A_{i}(k)-A_{i}^{\prime}(k)\right)^{2} \mathrm{~d} k
$$

This allows a second application of Parseval's theorem:

$$
E_{F, i}^{2}=\int_{n}\left(O_{i}(n)-O_{i}^{\prime}(n)\right)^{2} \mathrm{~d} n .
$$

Because for any $n$

$$
\left(O_{i}(n)-O_{i}^{\prime}(n)\right)^{2} \leqslant\left(O_{i+1}(n)-O_{i}^{\prime}(n)\right)^{2},
$$

it follows with equations 3.3.1 and 3.3.5 that

$$
E_{O, i}^{2} \leqslant E_{F, i}^{2} \text {. }
$$

With the same arguments for the focus plane $F$, clearly

$$
\left(F_{i+1}(n)-F_{i+1}^{\prime}(n)\right)^{2} \leqslant\left(F_{i}(n)-F_{i}^{\prime}(n)\right)^{2} .
$$


From this it follows with equations 3.3.2 and 3.3.5 that

$$
E_{F, i+1}^{2} \leqslant E_{O, i}^{2} \text {. }
$$

Combined with equations 3.3.7 we see

$$
E_{F, i+1}^{2} \leqslant E_{O, i}^{2} \leqslant E_{F, i}^{2}
$$

and

$$
E_{F, i+1}^{2} \leqslant E_{F, i}^{2} \text {. }
$$

Therefore the error decreases or stagnates with every iteration. This result proves the weak convergence, but does not guarantee that the algorithm always converges to a global minimum.

This is the reason why the HAAR algorithm is also discussed in this thesis. Based on the works of Haugazeau [Hau68] the HAAR algorithm is a strongly convergent algorithm for projections onto convex sets [BCL06]. The strong convergence properties depend on the assumption that the underlying sets of the projection $P_{o}$, the object-space amplitude constraint and $P_{F}$ the focus-space constraint are both convex.

The $P_{F}$ projection, which sets the phase component to zero or in other words, the projection from the set of all complex images onto the set of images with only real and positive images shall be discussed first. For this, we need to show that the set of real and positive images $S$ is indeed convex. Applying the definition of convexity this means for all images $s, t \in S$ and $\tau \in \mathrm{R}$ that

$$
\tau s+(1-\tau) * t \in S \text {. }
$$

This means that any linear combination of $s, t$, graphically represented by a connection line, must be in $S$.

Since only finite discrete images of two dimensions are of interest, we set the image size to $n \times n$ with $n \in \mathrm{N}$. Furthermore, since addition and multiplication with a scalar are applied element by element this discussion can be focused on single elements or pixels in this case. Therefore, we need to show that convexity applies to single pixels.

Let a single pixel in a real positive image be $p \in \mathrm{R}^{+}$. Since the convexity of the real numbers is a given, the set of positive real numbers is also convex and thereby the set of real positive images is convex as well. Thus, the magnitude projection of the focus plane constraint is convex.

The HAAR algorithm is strongly convergent [BCL06], but relies on the 


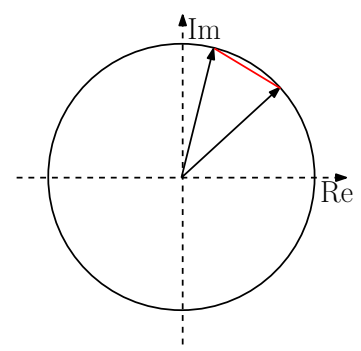

Figure 3.4. Non-convexity of the image plane constraint. The circle show the set of points with identical amplitude and the arrows highlight the phase of two examples. The red connecting line is their linear combination.

assumption that both projections are projections onto convex sets. For the wavefront correction problem, we have projections $P_{o}$ and $P_{F}$, the image plane constraint and the focus plane constraint.

For the image plane constraint, that we implemented by enforcing the measured amplitude and leaving the phase as it is, the situation is not so simple. We observe that the phase retrieval problem exhibits the same constraint and thus we can use the arguments made in [Mar07b] for phase retrieval.

If we visualize this projection onto a given amplitude in a plane of complex numbers, the projection set can be visualized by a circle. The instance of a real positive image is a point in this space. The radius is defined by that amplitude and the points on the circle are defined by different phase angles. As seen in Figure 3.4 the connecting line between two points of this sets is not inside the set, which means that this set is not convex. Therefore, image restoration with wavefront correction in projective formulation is not convex and the WFC-HAAR algorithm is not strongly convergent. Nevertheless, we can benefit from the very good convergence properties as will be seen in the results.

\subsection{The WFC-FISTA Algorithm}

Our literature review of incoherent deconvolution algorithm has shown that many algorithms gain their performance from appropriate priors. 
In the following section, we will derive a formulation of WFC suitable for proximal algorithms and show how to apply the FISTA optimization algorithm. For the application of proximal algorithms, we need to see the problem of wavefront correction from an optimization perspective. We regard the mean squared error between a blurred version of the optimized amplitude and the input amplitude.

Let $\mathbb{F}$ be the matrix that applies the Fourier transform by left multiplication and $O$ the image plane light distribution with dimensions $m \times n$. Thus, the complex aperture plane image $A$ is formed by

$$
A=\mathbb{F} O,
$$

using the same convention as in the other WFC algorithms. Now $\phi_{s a}$ corresponds to the wavefront deformation of the optical aberration, in contrast difference to the $\phi_{s}$ used for the projective WFC algorithms $\phi_{s a}$ is not written in multiplicative form(see Equations 3.1.2 3.1.3). It is embedded in the measured amplitude distribution $O_{0}$ and needs to be applied by using the Fourier transform, element-wise multiplication and inverse Fourier transform.

Furthermore, to revert to the image plane the inverse Fourier transform is applied. Hence, the transformation matrix $W$ from focal to image plane reads:

$$
W=\mathbb{F}^{-1}\left(\mathbb{F} \otimes \phi_{s a}\right) \text {. }
$$

This matrix $W$ maps a sharp image to its disturbed equivalent by left multiplication and we write $W(x)$ for the function that applies this linear transformation on an image $x \in \mathbb{C}^{m \times n}$. Nevertheless, this transformation does not include the image plane constraint yet. This step requires that the amplitude in the image plane is set to the measured amplitude $O_{0}$. To calculate the amplitude we require the absolute value of $W x$ and define function $W_{f}$ applied on image $x$ as:

$$
W_{f}(x)=|(W x)| \text {. }
$$

With this function $W_{f}$, the difference or error between the constraint and image $x$ sets an objective function with image plane constraint and can be measured by computing the $L_{2}$-norm element-wise:

$$
\left|W_{f}(x)-O_{0}\right|_{2} \text {. }
$$


Computing the $L_{2}$ norm means that the squared difference for every matrix element is calculated and then the square root of the sum is taken.

In this objective function, a regularization can be easily added. To minimize noise and ringing we choose the total variation prior, which enforces the sparsity of the image gradients. The intuition behind gradient sparsity is that images with large uniform patches separated by sparse strong edges should be favored over noisy images without structure. This idea is backed by natural image statistics as confirmed in [LFD+07].

This is accomplished by adding a term dependent on the gradient of $x$ weighted by $\gamma$. The gradient matrix of $x$ should be sparse, i.e. that few elements in the matrix have high values. While the idea of sparsity intuitively implies the $L_{0}$ norm, the $L_{1}$ norm is used instead, because it can be calculated much easier. The $L_{0}$ norm calculates the number of non-zero elements in the matrix, while the $L_{1}$ norm calculates the sum of all absolute values. The good properties of the $L_{1}$ norm as an approximation to $L_{0}$ are shown in [CP11]. Therefore, we use as regularization that the $L_{1}$ norm of the gradient of $x$ over the entire image is low. Throughout this thesis, unless noted otherwise, we use a gamma value of $5 \cdot 10^{-3}$, which is the same value as used in a FISTA code example by the authors of [FBA09].

All $L_{2}$ and $L_{1}$ norms in the following are applied element-wise. $O_{0}$ is the measured amplitude distribution in the disturbed image plane and focus plane image $x \in \mathbb{C}^{m \times n}$. Then the optimization target variable is the complex distribution in the focus plane $x$, which by focus plane constraint is forced to have a planar wavefront and therefore to be in $\mathbb{R}_{+}{ }^{m \times n}$ :

$$
\begin{aligned}
& \quad \arg \min _{x}\left\|\left(W_{f}(x)-O_{0}\right)\right\|_{2}^{2}+\gamma\|\nabla x\|_{1} \\
& \text { subject to: } x \in \mathbb{R}_{+}^{m \times n}
\end{aligned}
$$

Before the actual implementation, some more attention on prerequisites is required. Attempting to restore an image with $n \times n$ pixels results in an optimization target with a total variable count of $n^{2}$. The Jacobian matrix then has $n^{4}$ elements. Computing this number of elements of this matrix is both time and memory intensive. For an image with $1024 \times 1024$ pixel of double precision values, this means a Jacobian matrix would have the size of $1024^{4} \cdot 64$ bit. In contrast, the evaluation of the gradient evaluated in a certain direction, requires only the amount of storage as the image 


\subsection{The WFC-FISTA Algorithm}

itself, which is $n^{2}$.

A naive approach is to use a non-linear blackbox optimization algorithm to solve this problem. The Newton-Raphson algorithm is such a classic optimization algorithm, defined by its very fast quadratic convergence. The key element is that it requires the calculation of the Hessian matrix and its inverse in every iteration. With $n^{4}$ elements for computation and storage of this matrix the same argument as for a Jacobian matrix must be made, hence an alternative is necessary.

A way to solve this problem is the limited memory Broyden-FletcherGoldfarb-Shannon (L-BFGS) algorithm, a quasi-newton algorithm. It works similar to the gauss-newton method, but does not fully compute the Hessian matrix, instead it approximates it from past iterations. The specialty of the L-BFGS algorithm over other quasi-newton algorithms is that the Hessian matrix is not stored in full, but a low rank approximation with limited memory footprint is used instead.

Practical tests of applying this L-BFGS optimization algorithm on this objective function to restore images showed that this direct approach is very slow and does not converge to plausible results. This is unsurprising, because of the non-convexity of the problem.

Instead, we want to improve on this using the fact that the objective function is a compound. To reach a faster and better solution we want to apply the particularly fast fast iterative shrinkage algorithm (FISTA) as defined in Section A.7. Note the FISTA requires convexity of both parts. In the first step the objective function is split in two additive parts $f_{w f c}(x)+g_{w f c}(x)$. With

$$
f_{w f c}(x)=\left|\left(W_{f}(x)-O_{0}\right)\right|_{2}^{2}
$$

and

$$
g_{w f c}(x)=|\nabla x|_{1} .
$$

For the total variation term $g$, we apply the proximal map as in e.g. [BT09b].

Because $W_{f}$ can be expressed as a left multiplication with an invertible matrix $W$, a linear transform $p_{d}$, and an absolute value projection, it is clear that $f_{w f c}$ is convex. Thus with this different view of wavefront correction we gained convexity, which means that the requirements on $f_{w f_{c}}$ that FISTA demands are fulfilled. 
For the minimization of the quadratic term, FISTA requires the gradient of $f_{w f c}$. The derivative of the linear part of $W_{f}$ is easy to calculate and constant, but the absolute value function complicates it. When convergence is reached, the image should be real and positive. If the image is already real and positive, than the values remain unchanged by the absolute value function and it can be neglected. In the other case, we can use an approximation for the derivative by calculating the derivative without absolute value function influence and then project it onto the real values by applying the real part function Re. Hence in the next optimization step, only real values can occur.

Thus, the gradient of $f_{w f_{c}}(x)$ can be calculated very fast and easy, as if the absolute value function had no impact on it and $W_{f}$ were only a matrix multiplication with $W$ :

$$
\left.\nabla f_{w f_{c}}(x)=2\left(W^{T}(W x)-O_{0}\right)\right),
$$

then we add the real part functions to it:

$$
\nabla f_{w f_{c}}(x)=2 \operatorname{Re}\left(W^{T}\left(\operatorname{Re}(W(x))-O_{0}\right)\right) .
$$

Using this approach of approximating the gradient, we can apply FISTA very similarly as it is done for incoherent deconvolution in [BT09b]. We see that although the physical background and arguments differ, similar optimization problems must be solved.

As noted above, the allocation of matrix $W$ for an image of size $n \times n$ requires $n^{4}$ elements. Moreover, matrix multiplication is of higher computational complexity than fast Fourier transform. Thus, in the implementation of the algorithm, the steps to create it are applied separately on any given $x$ with the fast Fourier transform.

An overview of the algorithms used and developed in this thesis is shown in Table 1.2.

\subsection{Internal Patch Recurrence WFC}

For natural images the principle of local self-similarity can been observed using statistics [SI07]. This principle describes a fractal like quality of natural images, that for image elements of a high-resolution image, similar 


\subsection{Internal Patch Recurrence WFC}

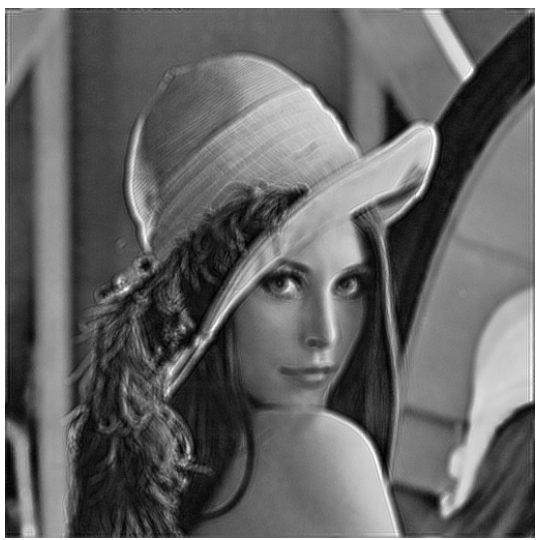

(a) Full scale

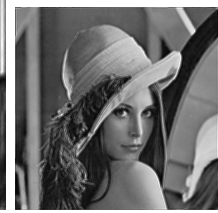

(b) 3x smaller

Figure 3.5. The same blurred image at different scales. The right image is sharper, because the width of the blur effects is scaled together with the image.

elements can be found in the low scales in the same image. Of course, images where this principle applies less can also be found, but for natural images the principle generally applies [SI07].

Moreover, images in smaller scale are generally sharper than high resolution images because the width of the blur is scaled with the image, see Figure 3.5, the same slightly blurred image is shown at two different scales. The right image is sharper, because the blur is not spread as wide as on the left image. Hence, the high resolution image is improved in sharpness by using matching elements from the small scale image.

Self-similarity has been successfully applied in recent works in computer vision on dehazing [BI16] and blind deconvolution [MI14]. In this section we want to derive an algorithm that applies this principle for wavefront correction, with the side goal of making this algorithm robust against noise.

Local self-similarity should act as a regularizer and enable a solution of the wavefront reconstruction problem that is not only plausible according to the scalar diffraction theory, but also gains in quality by exhibiting this property. 
WFC-AP algorithm is the most robust (see results for noisy input images in Section 4.2.2) projective algorithm discussed, thus we decided to use it as the conceptual foundation of this algorithm.

Inspired by [MI14] we enforce the regularization property of selfsimilarity as internal patch recurrence. This means that we apply the similarity property on small patches from a high resolution image and look in the low scale version of the image for sharper, low scale patches of the same content. The regularizer is then implemented as a replacement function of the blurred high res patches with low scale sharp variants. To find these patches, patches from the low scale image are sorted in a lookup database. For all image patches from high resolution image the most similar patch from the low scale database is found and then it is replaced by this patch.

To assemble the low scale patch database, a low scale image, that is sharper, than the original image must be produced. A special resizing function based on the sinc function is used, that as proven by [MI14], is theoretically best for recovering high frequencies in low scale images.

In the WFC-AP algorithm two projections $P_{o}$ and $P_{F}$ are applied on the object space image:

$$
O_{i+1}(n)=\frac{1}{2}\left(P_{o}\left(O_{i}(n)\right)+P_{F}\left(O_{i}(n)\right)\right),
$$

This internal patch recurrence regularizer only makes sense for images that are intended to be sharp. We apply it in the virtual focus plane of the projective algorithm. Thus, the first projection $P_{0}$, which enforces that the amplitude matches the measured amplitude is still necessary and remains unchanged. We want to adapt the second projection $P_{F}$, which enforces focus plane phase constraint, to use the internal patch regularizer, because the focus plane the image should be sharp and therefore have a high fractal self-similarity.

Therefore, with function SelfSim that replaces the input image with its more self similar counterpart, we define the projection on an object space image $O$, with variables as defined in Section 3.1.1 and 3.2:

$$
P_{f S S}(O)=\mathcal{F}^{-1} \phi_{s} \mathcal{F}\left(\operatorname{SelfSim}\left(\left|\mathcal{F}^{-1} \mathcal{F}(O) \phi_{s}^{-1}\right|\right)\right),
$$

or more directly this is the same $P_{F}$ as in Section 3.2 with an additional 
application of SelfSim after the focus plane constraint. Thus the WFC-SS algorithm is defined by:

$$
O_{i+1}(n)=\frac{1}{2}\left(P_{o}\left(O_{i}(n)\right)+P_{f S S}\left(O_{i}(n)\right)\right) .
$$

The application of internal patch recurrence requires some parameter tuning. The two most powerful parameters are the patch size and the scaling factor between high resolution and low scale image. Larger patches leads to a stronger regularization, but may remove details, while a large scaling factor gives sharper patches for replacement but may also remove details.

Because we do not want to fine-tune the parameters of the self-similarity prior for a specific dataset, but instead have an algorithm that works on any image, for both parameters we chose the same values, which other researchers [MI14] have also reported as successful. Note that they did not offer any explanation besides that these parameters work. Thus, we also set the size of the patches to 5 pixel and the scaling factor between high resolution and low scale image to 1.33 .

\subsection{Convergence Criteria}

In the previous section different WFC algorithms were introduced. The WFC algorithms are iterative algorithms that gradually restore the image. They can be stopped when the image is restored. To determine when this point is reached, a stopping criterion must be defined. For this decision about when to stop the algorithm, a convergence criterion can be used. General mathematical convergence occurs if only infinitesimal (up to computational accuracy) changes occur between iterations. This is not practical for actual image restoration with often imperfect and noisy input data, because sometimes many iterations are needed for the last bit of accuracy. Hence, it is difficult to design a convergence criterion which would reliably recognize the moment at which more iterations would lead to only slight improvement at the cost of large additional computational time. Therefore and also for the easy comparison between algorithms, it makes sense to stop after an arbitrary number of iterations. 
This has also the advantage that the execution time is predefined, because the number of iterations is constant. However, because the number of iteration must be predefined, it requires experimental experience about how many iterations are required to achieve a good-enough result, practically a safe upper bound will be taken. Certainly this can be optimized on an empirical basis, but it is still inefficient.

It does have the advantage of allowing a direct comparison between two different algorithms, because only the results must be compared. Thus, we use this criterion for the analysis of the different WFC algorithms in results Chapter 4. The algorithm is stopped, if the iteration index $i$ is greater or equal to the predefined number of intended iterations $n_{i t s} \in \mathrm{N}$ :

$$
i \geqslant n_{i t s}, n_{i t s} \in \mathrm{N} \text {. }
$$

For a more sophisticated convergence criterion, the actual image must be regarded. If algorithms are to be tested on images with simulated blur, the original reference images are still available. In this case, if such a reference image or other a prior information about the image is available, it can easily be used to determine a point at which the algorithm should be stopped. This point is reached, when a threshold similarity between the focal image of the current iteration and reference iteration is reached. Thus a comparison operation between restored image and reference image directly yields the criterion at which point to stop. This difference can be calculated in a number of different ways. The most direct choice is the sum of squared differences between the square of current iterations virtual focus plane intensity $F_{i n t}$ and the reference intensity $F_{r e f}$.

If this value is below a threshold $d \in \mathbb{R}$, the convergence criterion is triggered and we stop the iterations:

$$
\sum\left(F_{i n t}^{2}-F_{r e f}\right)^{2}<d
$$

The disadvantage of this basic method is that this difference is image brightness dependent. A better way is to check for a minimum PSNR (peak signal to noise ratio) value. PSNR is a standard measure of similarity between two images. For an introduction on how this value is calculated, see Appendix A.4. We stop the algorithm, if the PSNR value between the virtual focus plane intensity and the reference intensity is below a certain 
threshold $d$ :

$$
\operatorname{PSNR}\left(F_{i n t}^{2}, F_{r e f}\right)<d .
$$

In most application cases, such a reference image is not available. There is a given image and an aberration to be removed. In this case the only information available are the different intermediate images that were produced in previous iterations of the algorithm. As the WFC algorithms are very new, more empirical results need to be collected before a reliable convergence criterion can be designed.

\subsection{Finding Optimal Aberration Parameters}

The algorithms described in Chapter 3 require the wavefront deformation as an input. This means that for the restoration of a blurred image, the aberration parameters must be known. In general, wavefront deformation can be described with Zernike polynomials. In microscopy the lens itself is typically known and the the most relevant changing and unknown part of this aberrations is defocus, the same applies to the shadowgraph imagery (see Section 2.4).

This section shows an adaptation of the algorithm, where the deformation parameters are unknown. The goal is to estimate the spherical wavefront deformation which results in the sharp image. The search of the optimal aberration parameters can now be seen similar to an autofocus problem or as an optimization problem for maximum image sharpness. In Appendix A.1.4, we derive that the optical path length difference introduced by a spherical lens is equivalent to the wavefront deformation of defocus. Physically the radius of the wavefront in defocus depends on the distance to the sharp focus plane.

Algorithms solving this autofocus problem and giving an estimate of the defocus are known as 'autofocus algorithms' or focus criterions. From related work we see that autofocus algorithms are well studied field, because these algorithm have an important application as focus controllers in hand-held digital cameras. Autofocus algorithms try to find the configuration with the least blur based on a criterion of sharpness or respectively blur, which determines the sharpness of an image either absolutely or relatively to another images. [MXV14] classify in an extensive evaluation 
the different kinds of algorithms for autofocus in derivative based, image statistics based and histogram based. In [SDN04] an even larger overview over autofocus algorithms especially in microscopy is given. Particularly important to this work is the comparison of 18 different algorithms in 10 different microscopic settings each, with several configurations and optional preprocessing, such as low pass filtering by Sun et al. The conclusion of this experimental research with different image sets is that the overall best formula for measuring defocus is the normalized variation of pixel-values in the image. The definition of this criterion for image $I$ with height $h$, width $w$ and mean pixel value $\mu$ is as follows:

$$
F_{\text {variance }}=\frac{1}{h \cdot w \cdot \mu} \sum_{x=1}^{h} \sum_{y=1}^{w}(I(x, y)-\mu)^{2} .
$$

Note that these evaluations were all carried out on incoherent images and therefore the criterion needs to be tested for the coherent case and the WFC algorithm.

We have implemented the criterion in Equation 3.7.1 as recommend by [SDN04] and tested it on coherent shadowgraph images. The results with an evaluation and analysis are shown in Section 4.4.2. To summarize the results briefly at this point, this criterion does not work for shadowgraph images and a more specialized criterion is necessary. The reason is that in defocused coherent images, ringing occurs, together with high frequency interferences around sharp edges, which leads to false results.

For the application on coherent shadowgraph images, we propose a different criterion based on the local gradient and exponential pixel intensity. The reasoning behind this criterion is, that while sharply delimited plankton objects may show intensity gradient, they are still darker than the background, where the gradients should be low in this regions with high image intensity. For more details see Section 4.4.2. The currently used formula for focus estimation was developed empirically:

$$
F_{s}=\nabla I+\exp (I) .
$$

The criterion is then applied to every pixel and the sum is used as a measure for the entire image.

We refer to Section 4.4.2 for the results, which are better than the 
general criterion of [SDN04] and shows a strong local minimum for the correct parameters; however this minimum is not global. The robustness seems low and heuristics are necessary (see Figure 4.47). Therefore, we conclude in Section 4.4.2, that these hand-crafted criteria are not sufficient for our intended application on coherent shadowgraph images. We seek a criterion, which is insensitive against possible restoration artifacts and robust enough for the application on real images. This motivates our development of a specialized criterion with deep learning methods in Section 4.4.3.

\subsection{Large Image Processing}

Large images give researchers the ability to view a large field of view with a high resolution. For this reason in many applications such as in microscopy or astronomy, the processing of large images is becoming increasingly important. This is highlighted by the fact that today image sensors with over $20 \mathrm{MP}$ (megapixel) can be found even in consumer devices such as smartphones ${ }^{1}$ and are also increasingly common in industrial cameras ${ }^{2}$. For the shadowgraph described in Section 4.4 a 29 MP camera is employed.

In this section the properties of wavefront correction algorithms when employed on large images is discussed and strategies for lowering the runtime and memory requirements are introduced.

The key component of the WFC algorithms is the Fourier transform, which with a naive implementation has a computational complexity in $O\left(n^{2}\right)$ and with the Fast Fourier transform algorithm for inputs with $2^{n}$ elements [DV90] has a computational complexity in $O(n \log (n))$, with $n$ being the number of elements to be transformed or pixels of the image. Because this section is largely about runtime, we must also consider concrete possible implementation without losing generality.

If the width and height of an image is doubled, the number of pixels and thereby input array size for the Fourier transformations in the algorithm is quadrupled.

\footnotetext{
${ }^{1}$ Nokia Lumia 1020 with $41 \mathrm{MP}$ sensor

${ }^{2}$ Point Grey Blackfly S with 20 MP Sony IMX183 is announced for Q3 2017.
} 
3. The Wavefront Correction Algorithm

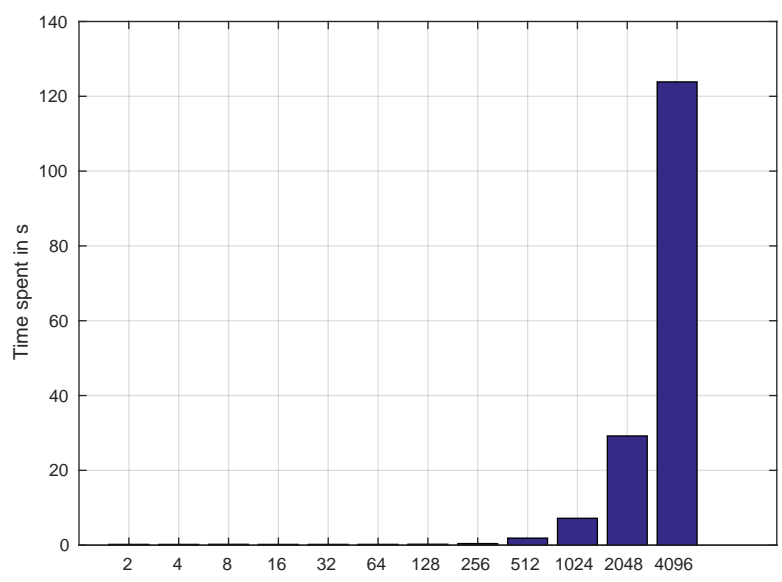

Figure 3.6. Runtime for per iteration WFC-GS for a square image with increasing width and height ( $\mathrm{x}$-axis).

As explained above the total runtime per iteration grows even faster with $O(n \log (n))$. Therefore, large image quickly become unpractical to handle as shown by Figure 3.6.

We propose a tile based approach, in which we divide the image into square tiles of the same width and height. Image padding is used for non-matching irregular image sizes.

A detrimental effect of this technique is that boundary ringing artifacts may be spread across the image. In the result Figure 4.21 the tiles used for large image processing are clearly visible. This motivates the next section about boundary ringing suppression.

\subsection{Boundary Ringing Suppression}

This section focuses on the suppression of ringing occurring in the borders of an image. A good example can be seen in Figure 3.7 with periodic structures of changing intensity extending from the image borders in 


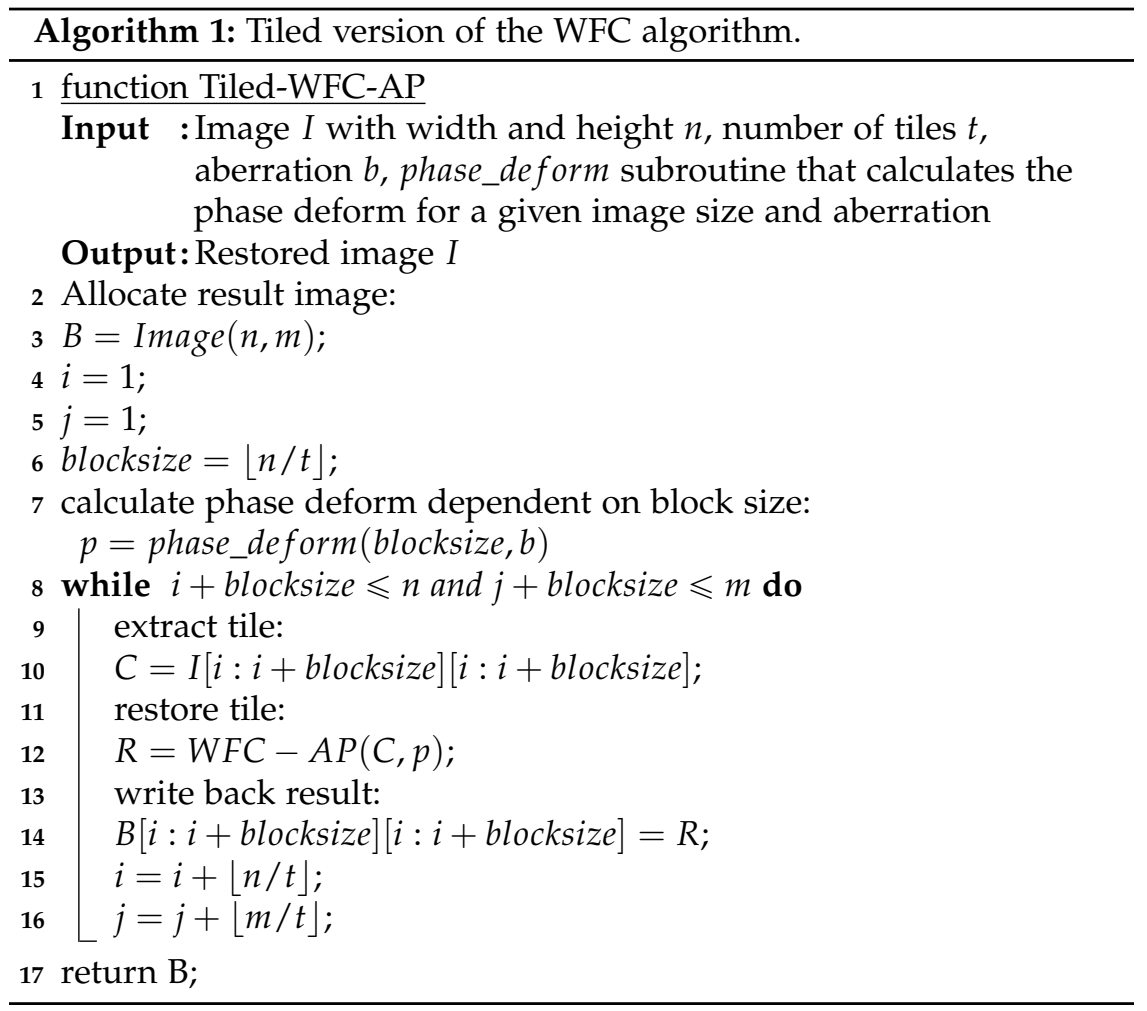

the restoration. The reason for this ringing can be found in the repeated application of the Fourier transform.

The Fourier transform in mathematics is defined as an integral over an infinitely long signal. If the Fourier transform is applied on a limited interval, ringing effects caused by discontinuities on the image border may occur. In the following different strategies to prevent or lower border ringing are presented and discussed. Note that a different type of ringing suppression occurs due to an imperfect imaging system and incomplete wavefront correction is discussed in the application on shadowgraph images in Section 4.4 .

In signal processing a typical way to reduce this problem is windowing. 


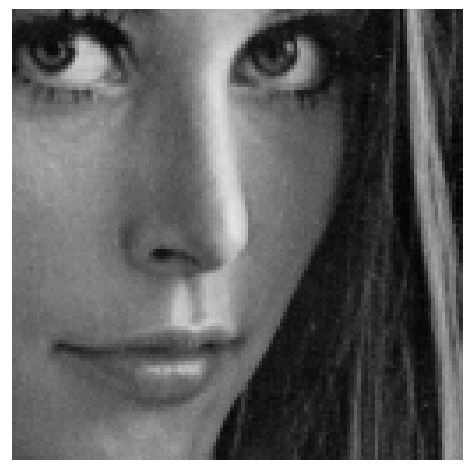

(a) Reference

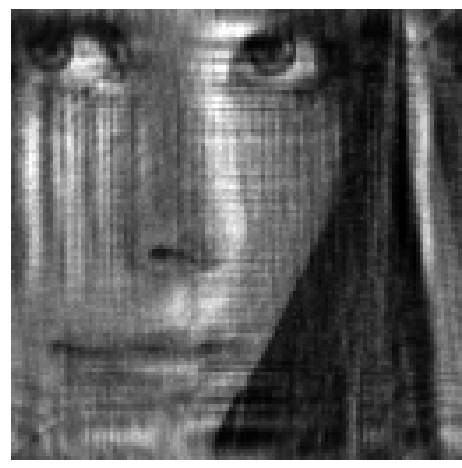

(b) Restoration

Figure 3.7. Example of image restoration with strong boundary ringing, produced by restoring a small defocused image with $256 \times 256$ pixels. The input image was created by cropping a larger image with simulated defocus. For more details, see Section 4.2.6.

Windowing works by lowering the amplitude of signals closer to the border and thereby reducing the influence of these parts of the signal which are responsible for the artifacts. Common functions used for modulating the signal amplitude are the Hanning and Hamming windows. This approach is clearly not usable for images as it drastically lowers image quality.

Similar in idea, but developed especially for images is edge tapering. Edge tapering is a technique which tries to lower the effects by smoothing the borders of the image. This causes a removal of highly frequent components in the border regions, which cause very visible artifacts as they introduce a strong break to the infinite image assumption.

Image padding in contrast does not change the image itself, but tries to achieve a higher repetitiveness of the image and thereby a higher compatibility of with the Fourier transform. The image is padded with values to achieve a smoother higher repetitiveness in the signal. The way this is done is important, as it directly decides the quality of the restoration. The process is illustrated for an image in Figure 3.8, the center image is the original image and the red border signifies the padded version, as padding size half of the image width/height is chosen. We use three different 


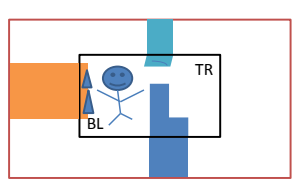

(a) Replicate boundary

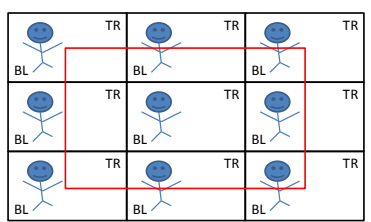

(b) Cyclic boundary

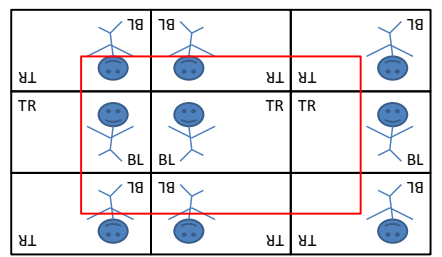

(c) Symmetric boundary

Figure 3.8. Illustration of replicate, cyclic and symmetric boundary condition with the original image in the middle and the new padded image within the red border.

padding techniques.

The first technique is called the replicate boundary condition and sets the value outside of the original image to the value of the closest pixel of this image. In the second technique, the original image is embedded into versions of itself, called the cyclic boundary condition. With this technique, a discontinuity occurs between one image and the next. This is rectified with the symmetric padding technique, which embeds the original image into flipped versions, so that no discontinuities occur, because the outer border of the image is always continued by a reflected version. $C_{0}$ continuity is achieved [VBD+05]. Antisymmetric padding goes a step further and enforces $C_{1}$ continuity by attaching inverted flipped versions [VBD+05].

For all these techniques, the new padded image replaces the original input image. This means that constraints of projective algorithms are enforced in the center original image and in the padded area. However the constraints, amplitude in the defocus plane and phase planarity in 
the focus plane, are only valid for an image and not necessarily for an image and some padding. Particularly the transition between center image and padding is not formed according to the image formation model, the coherent scalar diffraction theory.

Thereby, we propose an extension to the padding techniques discussed above, called 'partial enforcement of constraints'. With this technique, the WFC algorithms function as before, however in every step where constraints are enforced, they are restricted to the center image.

It is clear that the difference between repetitive border and infinite integral is image dependent and as such, an evaluation on typical examples is necessary. For the evaluation of the different strategies presented here we refer to the results section. Another important point to be made is that the size of the images also matter strongly. For small images, the theoretical difference and expected artifacts are much higher than for a large image. This effect and the boundary ringing suppression techniques introduced above are analyzed in Section 4.2.6 of the results chapter.

\subsection{Deconvolution Algorithms on Coherent Im- ages}

As outlined in the introduction, we want to follow up with a detailed explanation why incoherent deconvolution algorithms cannot be used to restore coherent images. Given a sharp reference image, a coherent defocused image is formed with the following steps. First, the amplitude is calculated from the intensity of the sharp image $I_{i n c}$ :

$$
O_{a}=\sqrt{I_{i n c}} \text {. }
$$

This amplitude defines the entire image, because the reference image is sharp, its phase is planar and the entire wave field is set with phase $\phi$ :

$$
O=O_{a} \cdot \exp (\phi),
$$

where without regarding offsets we can set $\phi=0$. For a defocused image a spherical wavefront deformation $\phi_{d}$ in the aperture plane is added, which is reached via Fourier transform. Because we are interested in the image, 
we transform directly back into the image plane:

$$
O_{\text {dis }}=\mathcal{F}^{-1}\left[\mathcal{F}\left(O_{a}\right) \cdot \exp \left(j \phi_{d}\right)\right] .
$$

A hypothetical image sensor measures only intensity data $O_{S C}$ :

$$
O_{S C}=\left|O_{\text {dis }}\right|^{2} \text {. }
$$

This is the input for any restoration algorithms.

A direct deconvolution algorithm requires a PSF. With aperture $A$, it is formed by [MRC+01]:

$$
P S F=\left|\mathcal{F} A \exp \left(j \phi_{d}\right)\right|^{2}
$$

If we disregards any practical issues with noise or zero values in the PSF, which are solved with more sophisticated algorithms introduced in Section 2.2, we can apply direct inversion. This is the base of any deconvolution algorithm. The restored image $I_{r}$ is then formed by

$$
I_{r}=O_{S C} \otimes P S F^{-1},
$$

For incoherent images, it is clear that this generally (disregarding issues of PSF inversion) works, because with the incoherent image formation model for a defocused incoherent image $O_{S I}$,

$$
O_{S I}=I_{i n c} \otimes P S F
$$

the restored image $I_{r}$ (disregarding complications of PSF inversion)

$$
\begin{aligned}
I_{r} & =O_{S I} \otimes P S F^{-1} \\
& =I_{i n c} \otimes P S F \otimes P S F^{-1} \\
& =I_{i n c}
\end{aligned}
$$

is the intended sharp reference image. This is equivalent a Fourier transform variant with element wise multiplication

$$
I_{r}=\mathcal{F}^{-1}\left[\mathcal{F}\left(O_{S I}\right) \cdot \mathcal{F}\left(P S F^{-1}\right)\right] .
$$

and is in fact very similar to [PRF90], where the expanded term

$$
I_{r}=\mathcal{F}^{-1}\left[\frac{\mathcal{F}\left(O_{S I}\right) \mathcal{F}(P S F)}{(\mathcal{F}(P S F))^{2}}\right],
$$




\section{The Wavefront Correction Algorithm}

is used to account for zero values in the PSF.

If we apply the same deconvolution approach to the coherent case:

$$
I_{r}=O_{S C} \otimes\left(P S F^{-1}\right) .
$$

Substituting equations $3.10 .4,3.10 .5$ and 3.10 .3

$$
I_{r}=\left|\mathcal{F}^{-1}\left(\mathcal{F}\left(O_{a}\right) \exp \left(j \phi_{d}\right)\right)\right|^{2} \otimes\left(\left|\mathcal{F}\left(\exp \left(j \phi_{d}\right)\right)\right|^{2}\right)^{-1},
$$

we see that we cannot simplify the equation as before, because of the absolute value and square functions. Applying the Fourier convolution theorem, we can write this as a fraction:

$$
I_{r}=\mathcal{F}^{-1}\left[\frac{\mathcal{F}\left(\mid \mathcal{F}^{-1}\left(\left.\mathcal{F}\left(O_{a}\right) \exp \left(j \phi_{d}\right)\right|^{2}\right)\right.}{\mathcal{F}\left(\mid \mathcal{F}\left(\left.\exp \left(j \phi_{d}\right)\right|^{2}\right)\right)}\right] .
$$

However, the critical points, the absolute value and square functions, remain. We could only solve this problem, if these functions did not exist in $O_{S C}$. We cannot evaluate the $I_{r}$ without knowing the phase. This is physically impossible, because image sensors generally only acquire intensity information, from which the phase cannot be recovered. Therefore, we only have the value of $O_{S C}$ without its phase. The phase information is lost by these absolute and square functions.

To be able to remove these and to directly apply this inversion, we would require the phase deformation of the amplitude(square root) of $O_{S C}$. This is the motivation for the WFC-algorithm, which recovers both amplitude and phase from the measured blurred coherent intensity image by using an additional focus plane constraint.

Ignoring all these theoretical considerations, standard incoherent deconvolution algorithms can of course be applied to any image, the results are shown in Section 4.2.7. 


\section{Chapter 4}

\section{Applications and Results}

The first section of this chapter focuses on results on simulated images and is independent of the field of application. The second section demonstrates how to apply the WFC-algorithm on large images. Next, the third section focuses on the first field of application of the WFC-algorithm, the restoration of microscopic images and a verification of the simulation by comparison with microscopic imagery.

Finally, the fourth section shows the results of the application for a novel underwater optical sensor based on the shadowgraph and includes further advanced applications such as all-in-focus images from spatially variant aberrations and $3 \mathrm{D}$ visualization of depth from defocus.

\subsection{Implementation}

All algorithms were implemented in Matworks Matlab 2015b [Mat15] and use functions of the image processing and the parallel computing toolbox. The projection algorithms can be run multi-threaded on a CPU and on the GPU using the Nvidia CUDA ${ }^{1}$ framework. The WFC-FISTA algorithm was implemented for CPU based on code by [FBA09]. The artificial neural network for focus estimation were implemented with the Caffe framework ${ }^{2}$ and trained with the Nvidia DIGITS ${ }^{3}$ user interface. Using the Matlab interface for Caffe the model is applied on test images. For experiments with L-BFGS optimization an implementation by Mark Schmidt, University of British Columbia ${ }^{4}$ is used. Run times reported in

\footnotetext{
$1_{\text {https: //developer.nvidia.com/cuda-downloads }}$

2 http://caffe.berkeleyvision.org/

${ }^{3}$ https://github. com/NVIDIA/DIGITS

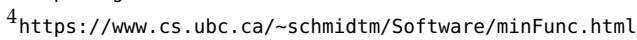




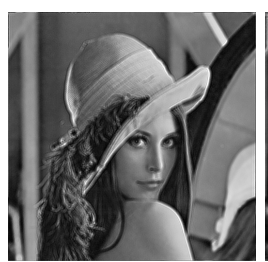

(a) $5 \lambda$

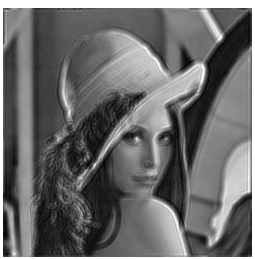

(b) $15 \lambda$

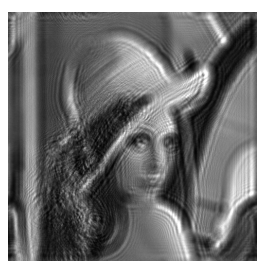

(c) $45 \lambda$

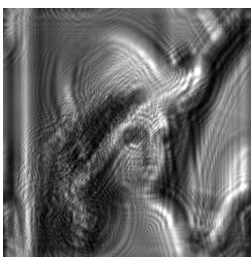

(d) $100 \lambda$

Figure 4.1. Defocus of increasing strength applied on an image, measured in maximum wavefront modulation in wavelengths.

this thesis are measured on a computer with a $4 \mathrm{GHz}$ Intel i7 processor $^{5}$ and a Nvidia GTX970 graphics card ${ }^{6}$.

\subsection{Results on Simulated Images}

In the previous chapters, different algorithms for solving the wavefront correction algorithm were presented.

To evaluate the performance of WFC it is applied to reference images with simulated aberrations. These references with simulated defocus are created based on scalar diffraction theory with simulated wavefront deformations in the aperture plane. After an introduction into coherent aberrations and some results of the WFC-GS algorithm to show the principle of wavefront correction, an analysis of the different algorithms follows.

In Figure 4.1 an image with different strength of defocus is shown. Strongly visible are the interference and ringing on edges, which is typical for coherent aberrations. With stronger defocus the image becomes unrecognizable to the eye.

Defocus can be seen as a spherical deformation of the wavefront. A more general description of wavefront deformations are the Zernike polynomials introduced in Section A.5, for defocus this leads to an equivalent

\footnotetext{
${ }^{5}$ detailed specification at:

https ://ark. intel . com/de/products/80807/Intel - Core- i7 - 4790K-Processor - 8M- Cache - up - to - 4_40- GHz

${ }^{6}$ detailed specification at: http://www.geforce.com/hardware/desktop-gpus/geforce-gtx-970/ specifications
} 
spherical polynomial term. Although defocus is the most common wavefront deformation, it is not the only kind of deformation; in fact the shape can be arbitrary. The Zernike polynomials have unique properties which are important in optics. The first terms have a direct relation to the classic Seidel aberrations, for example the 4. Zernike term (Noll index) represents defocus, the 5. and 6. astigmatism and the 7. and 8. coma. Such a spherical wavefront deformation is shown in Figure 4.2a, in which the wavefront is depicted as multiples of the wavelength perpendicular to the propagation direction. The corresponding image is shown in Figure 4.3a.

We start with a sharp image. Images are typically intensity images. Hence, the square root is calculated to gain the amplitude distribution. We consider this image as free of aberration and in the following steps shall compute a simulated image with an arbitrary wavefront distortion. Because it is a sharp image in a focus plane, the wavefront in this plane is planar and thus no phase information is required and we set it to zero. Constant offsets in the phase have no effect, physically because they do not influence the shape of the wavefront and mathematically, because the offsets they cause in the Fourier transform is irrelevant.

In the next step, this sharp amplitude distribution is Fourier transformed. The result is another amplitude and phase distribution. Because a lens in a focused optical system has the same effect, this can be seen as the corresponding aperture plane distribution.

To create the defocus effect, a sphere with certain radius, which defines the wavefront, is added to the phase of the Fourier transformed image. The radius determines the severity of the defocus, larger radii lead to a smaller defocus. The phase values from the Fourier transform are limited from 0 to $2 \pi$, other phase values are wrapped to this range.

This is illustrated in Figure 3.1 where the image plane is moved from the focus plane. To incorporate this shift, an additional wavefront deformation is added in the aperture plane of an optical system. We assume that the defocus is known and compensate it with adequate spherical aberration. This additional phase shift has the same effect as changing the shape of the lens. In the next step, with the inverse Fourier transform a defocused image amplitude is computed. To simulate a real light sensor, such as a camera, the intensity image is calculated by squaring the amplitude values. 

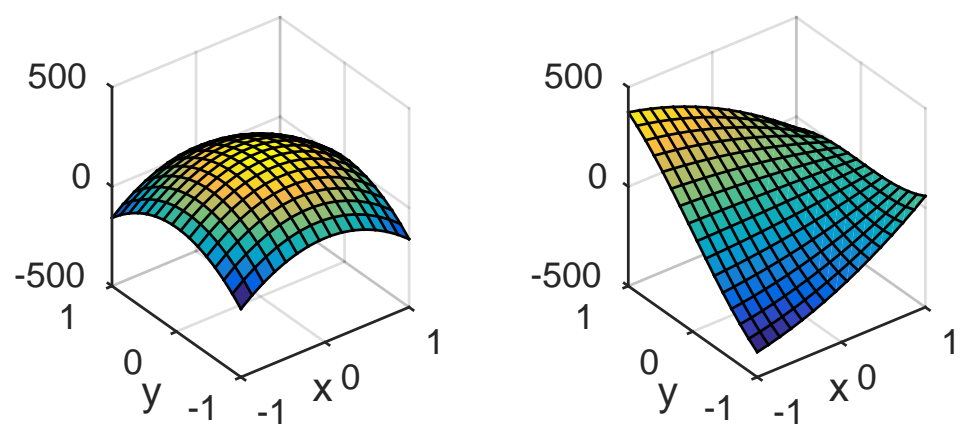

(a) Spherical wavefront deformation (b) Arbitrary wavefront deformation

Figure 4.2. Visualization of wavefront deformation in multiples of wavelength over the $x-y$ aperture plane.

The same computation of distorted images can be performed for any wavefront distortion, not only for defocus. As outlined above, it is advantageous to describe the wavefront with Zernike polynomials, as it is common in optics.

Such a wavefront deformation consisting of a combination of defocus, astigmatism and coma is depicted in Figure $4.2 \mathrm{~b}$. An image disturbed by this deformation shows the typical ringing and interference effects, but they are no longer circularly symmetric as in an image disturbed by a purely spherical wavefront (Figure 4.3a).

Wavefront deformation is usually expressed in units of wavelength across the aperture. Nevertheless, the strength of an aberration depends linearly on the optical path length difference induced by the wavefront deformation and on the size of the aperture. Hence, we arbitrarily set the aperture size to the image size, which is in most cases, unless specified otherwise $512 \times 512$. With this conventions the wavefront shown in Figure $4.2 \mathrm{~b}$ is defined by the sum of the Zernike polynomials with ANSI standard Noll index notation (see Appendix A.5 for definitions):

$$
W_{\text {arb }}=-117 Z_{4}+-100 Z_{5}+-75 Z_{7}
$$

whereas the strong spherical wavefront deformation in Figure 4.2a is 


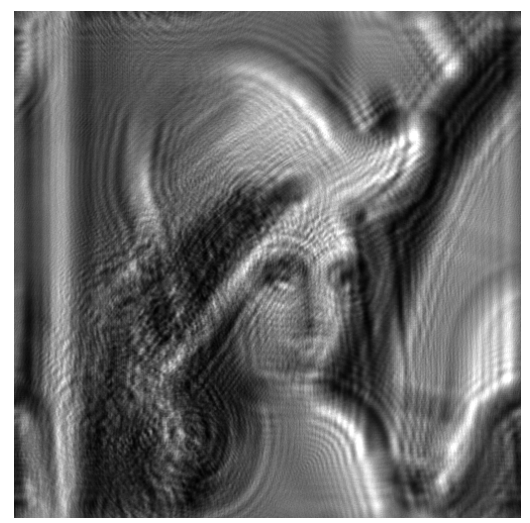

(a) Image with wavefront deformation in Figure 4.2a

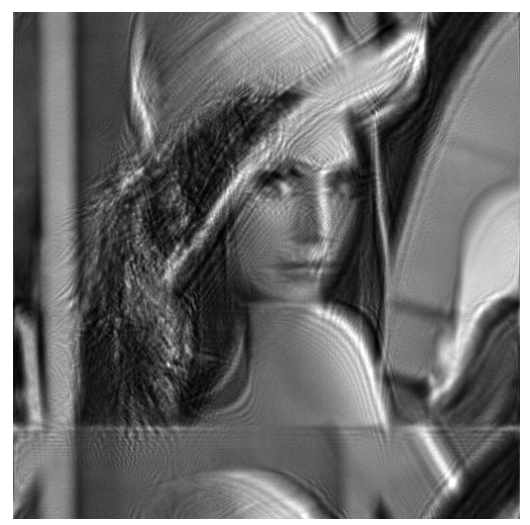

(b) Image with wavefront deformation in Figure $4.2 \mathrm{~b}$

Figure 4.3. Image with different wavefront deformations. The visible wraparound in image (b) is due to the very strong asymmetric deformation and the repetitiveness of the Fourier transform.

defined by:

$$
W_{s}=-117 Z_{4}
$$

For defocus in this thesis a more direct description using the maximum optical path length difference is used. Because it is linear dependent on the assumed wavelength, we set a simulation wavelength of $\lambda=2 \pi$. This means that the optical path length difference of $W_{S}$ is:

$$
\begin{aligned}
& \max \left(W_{S}\right)-\min \left(W_{S}\right) \\
& =\max \left(-117 Z_{4}\right)-\min \left(-117 Z_{4}\right) \\
& =\left(-117 \sqrt{3}\left(2 \cdot 0^{2}-1\right)\right)-\left(-117 \sqrt{3}\left(2 \cdot 1^{2}-1\right)\right) \\
& =2 \cdot 117 \sqrt{3} \\
& =65 \lambda
\end{aligned}
$$

for an aperture of size $\frac{512}{2 \pi} \lambda=81 \lambda$. For images not of size $512 \times 512$, values are adjusted so that the strength of defocus effect is equivalent, so that we retain comparability and applicability in several scales. This is important 
4. Applications and Results
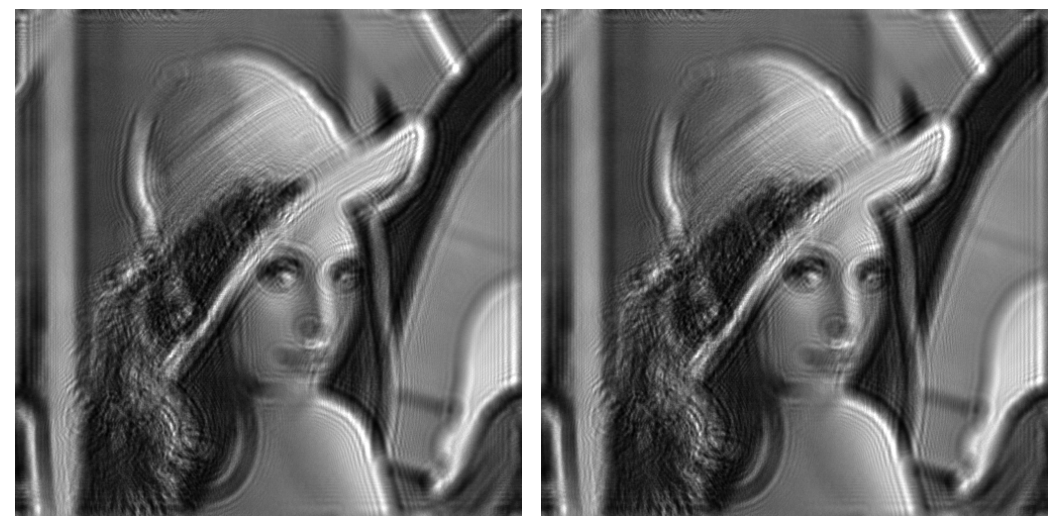

(a) Defocus with maximum wavefront deformation of $25 \lambda$

(b) Defocus with maximum wave-

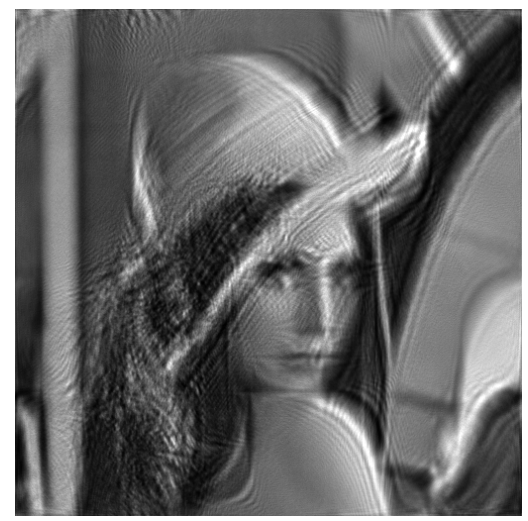
front deformation of $-25 \lambda$

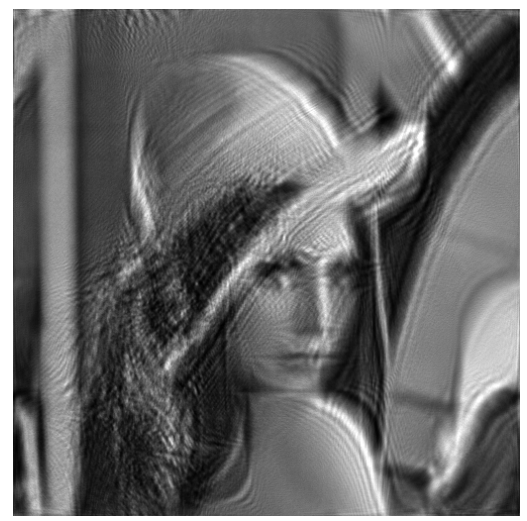

(c) Strong astigmatic wavefront defor- (d) Inverted strong astigmatic wavemation front deformation

Figure 4.4. Symmetry of defocus. 


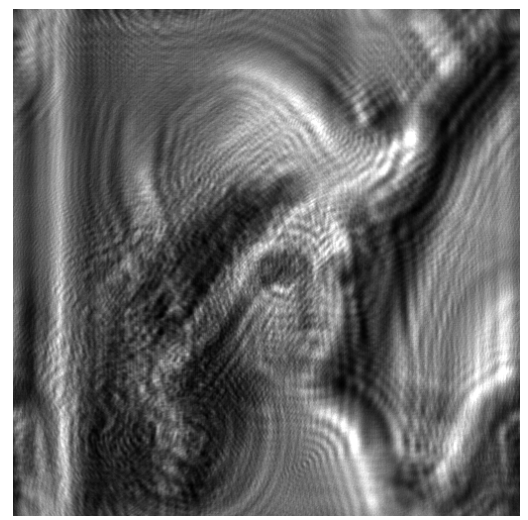

(a) Input image

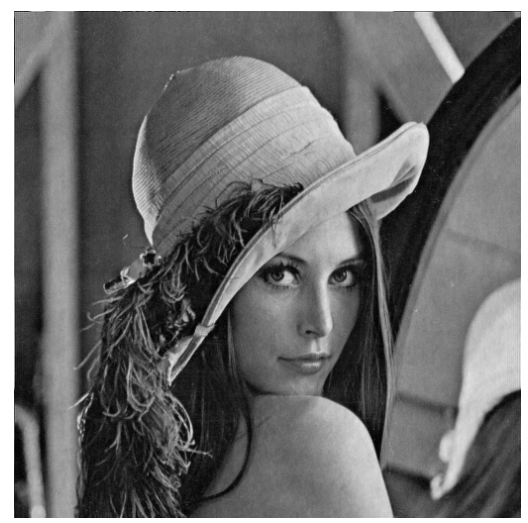

(b) Restoration with WFC

Figure 4.5. The Wavefront Correction Algorithm restores images disturbed by strong defocus.

for the correct compensation at differences scales of the WFC-SS algorithm.

Another point to make is that the effect of optical aberrations is symmetric. In Figure 4.4 two images with identical but inverted spherical wavefront deformation are shown. As can be seen, they are identical. The consequence is of major importance for the wavefront correction, because this means that the sign of the deformation is inconsequential. Therefore, for the compensation of for example defocus effects it is not necessary to know whether the image plane was in before or behind the focus plane at the time of acquisition. However, this also means that one cannot recover this information from the image.

In Figure 4.5 a first result of the WFC-GS algorithm is shown. It demonstrated that the algorithm recovers the sharp image from an image disturbed by simulated wavefront aberrations and is hence working as theoretically predicted. In the next step the algorithm is tested on the image sequence shown in Figure 4.1. The result is depicted in Figure 4.6. It shows a successful restoration even for details which are not recognizable for the eye.

To complete these tests, the WFC-GS algorithm is used on the images in Figure 4.3, which are generated with an arbitrary wavefront deformation 


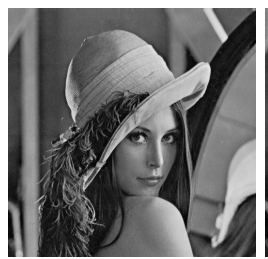

(a) $5 \lambda$

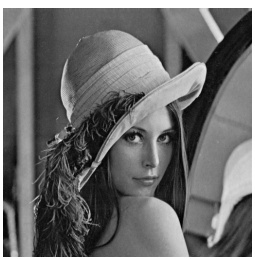

(b) $15 \lambda$

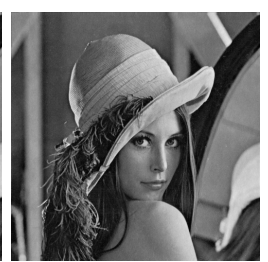

(c) $45 \lambda$

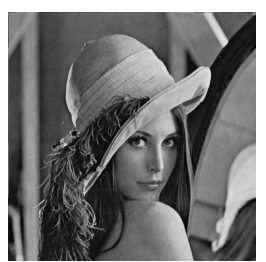

(d) $100 \lambda$

Figure 4.6. Correction of input images with increasing defocus in Figure 4.1.

shown in Figure 4.2. As the results show, the restoration is impeccable. Even the wrap-around in the lower areas of the image is corrected, because the same effect of the Fourier transform that causes the wrap-around in the simulated input images with strong asymmetric wavefront deformation, also applies during the restoration. Figure 4.7 demonstrates that the restoration is successful for different kinds of images. Both low and highly textured areas can be recovered well.

In conclusion the WFC algorithm is able to restore a sharp image even if the wavefront aberration is strong and asymmetric.

\subsubsection{Comparison with Related Work}

In Section 2.1 we introduced related prior works on coherent image restoration and discussed the shortcomings of their theoretical approach. In this section the discussed methods and the WFC-algorithm are compared by their direct results. An image with simulated aberrations (in this case a defocus) is prepared as a test subject, see Figure $4.8 \mathrm{~b}$. The state of the art methods [MKS+09],[KDL13] and WFC-HAAR are applied on it with the same correcting wavefront aberration.

The results are shown in Figure $4.8 \mathrm{~d}$ and Figure $4.8 \mathrm{c}$.

Clearly visible in Figure 4.8 is the large difference between the related work method [KDL13] [MKS+09] and wavefront correction. We will also compare the algorithms by their peak signal to noise ratio (PSNR) calculated from the ratio between the difference between original image and restoration from defocused image as noise and the original image as signal, as further explained in the Appendix A.4. The results of the PSNR value 


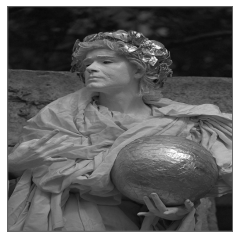

(a) Original ${ }^{1}$

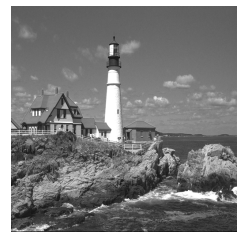

(d) Original ${ }^{1}$

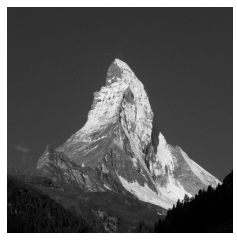

(g) Original ${ }^{2}$

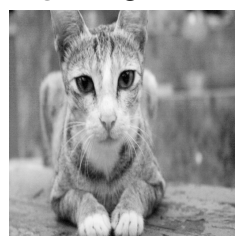

(j) Original ${ }^{3}$

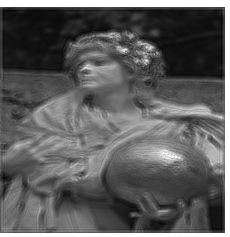

(b) Defocused

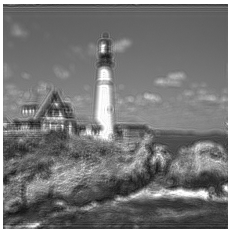

(e) Defocused

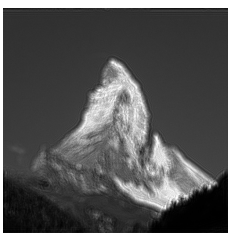

(h) Defocused

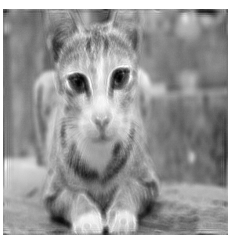

(k) Defocused

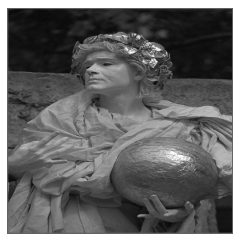

(c) Restored

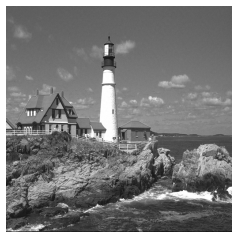

(f) Restored

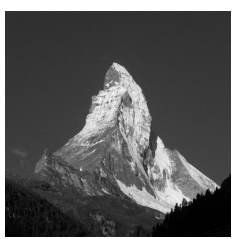

(i) Restored

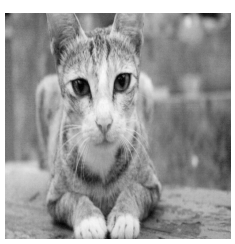

(1) Restored

Figure 4.7. Different images with simulated aberrations and restoration with 600 iterations of WFC-HAAR.

${ }^{a}$ Kodak PhotoCD test image dataset, 1991; Released for unrestricted use: http://rok.us/ graphics/kodak/

${ }^{b}$ Author: Wikimedia I Simplon2010; Public domain: https://commons.wikimedia.org/wiki/File\% 3Matterhorn_august_2009.JPG

${ }^{c}$ Author: Wikimedia IDll; Public domain: https://commons.wikimedia.org/wiki/File٪3ACat_ public_domain_dedication_image_0011.jpg 
4. Applications and Results

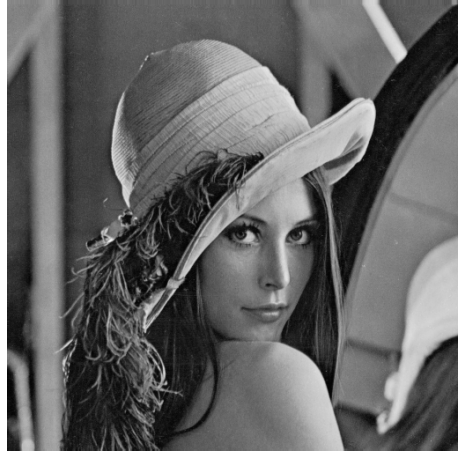

(a) Original image

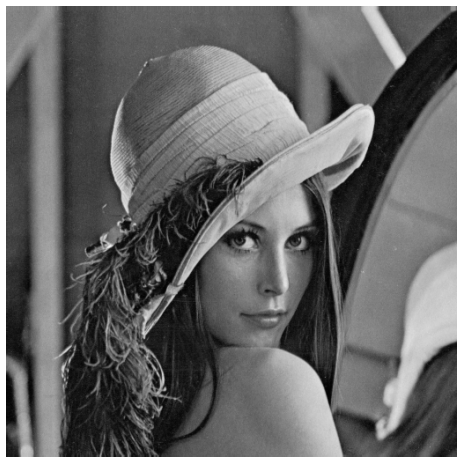

(c) Restored image with WFC- (d) HAAR PSNR 47.3dB

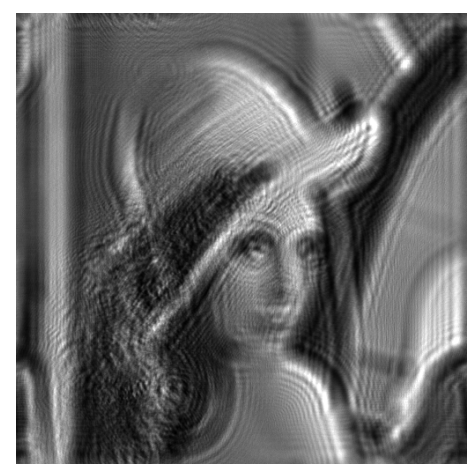

(b) Defocused image

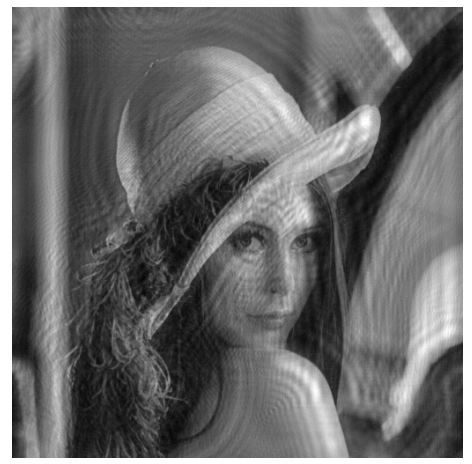

(d) Restored image with inverse filter method by [MKS+09] [KDL13] PSNR 23.9dB

Figure 4.8. Image restoration with wavefront correction and with related works inverse filter method. 
with $23.9 \mathrm{~dB}$ related work to $49.6 \mathrm{~dB}$ with WFC-HAAR confirm the strong visible differences and superiority of the novel method. Thus, [KDL13] and $[\mathrm{MKS}+09]$ are not included in further evaluations.

\subsubsection{Comparison and Analysis of WFC Algorithms}

Different projection based algorithms as well as the WFC-FISTA algorithm were presented in the main part of this thesis. In this section we will compare them with a detailed analysis. An overview of the different algorithms is given in Table 1.2.

In general, the wavefront correction algorithm works by removing the blur from an defocused image and thus restores the image to its full fidelity, see Figure 4.8. Speed and robustness are the two main criteria characterizing the performance of a good restoration algorithm. We will compare the algorithms by their peak signal to noise ratio (PSNR) calculated from the ratio between the difference between original image and restoration from defocused image as noise and the original image as signal.

Because of its strong self-similarity prior, the WFC-SS algorithm it is discussed separately in the section on the effect of noisy and imperfect input, Section 4.2.4.

The restoration quality with a given number of iterations is shown in Graph 4.9 as the PSNR to the ideal restoration. All tested wavefront correction algorithms achieve good results, the WFC-HAAR algorithm shows the best convergence, confirming the theoretical advantages. The WFC-RAAR and WFC-HIO algorithm have slower convergence and the WFC-AP algorithm is the slowest of the presented projection algorithms. The WFC-FISTA algorithm convergences very fast, but does not improve in PSNR from then on. This behavior is caused by the regularization, which inhibits this algorithm from reaching an exact restoration. However, this can become advantageous as the results on noisy data below show.

In the next step, we visually compare the results of algorithms with a fixed very low number of iterations. This very low number of iterations should make it easy to spot differences. The results for the entire image after 7 iterations are shown in Figure 4.10. Although the PSNR values differ much, the visual difference in restoration quality is not as prominent as expected. Clear edges such as the boundaries of the hat are sharply 
4. Applications and Results

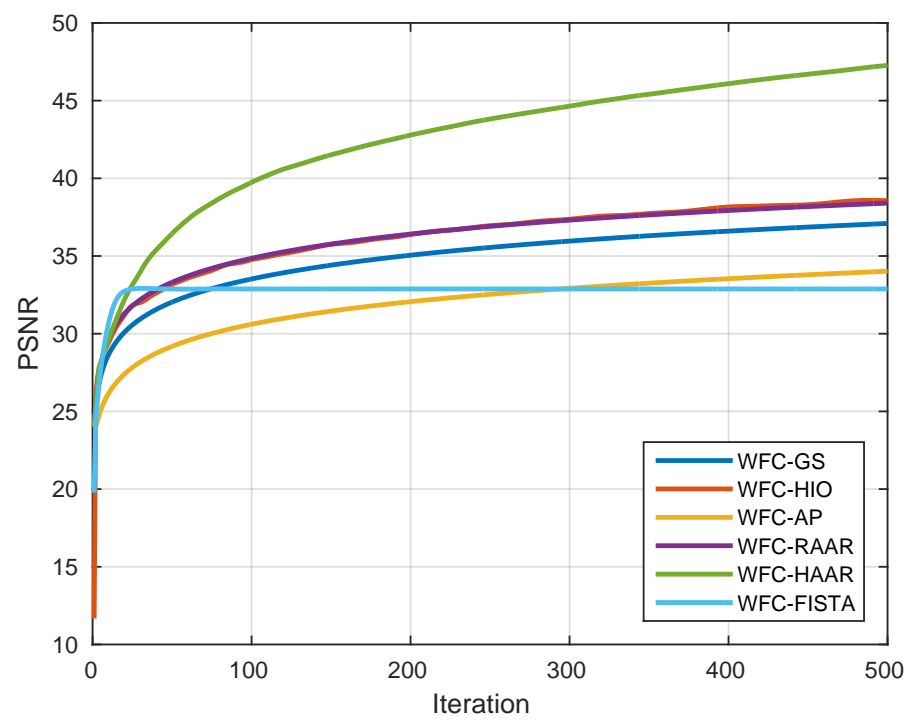

Figure 4.9. Comparison of the convergence of different WFC algorithms in $d B$ of PSNR.

restored with all algorithm. Most method differ mostly in the visibility of large scale ring-like intensity variation around sharp edges. One observation is that the frequency of these variation is higher for the restorations of measurably higher quality (compare WFC-AP and WFC-HAAR results), which seems counter intuitive.

This becomes clearer if we look at results from of the WFC-HAAR algorithm with different iteration counts in side-by-side comparison. The results are shown in Figure 4.11 and show the same effect of higher frequency artifacts in image restored with more iterations, until they vanish with very high iteration counts. It also demonstrates that visually pleasing restoration can be reached with few iterations and that it may not always be necessary to apply the algorithms until full convergence.

Overall, we spot the biggest difference in the restorations of the WFCHAAR and WFC-AP in clarity of the feather decoration of the hat. As next, we want to focus on small structures. A similar experiment to the previous 


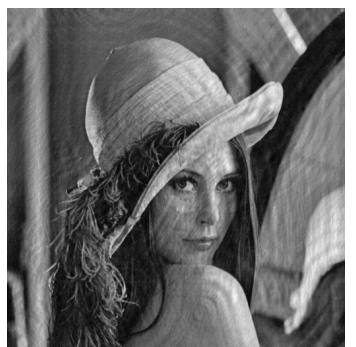

(a) PSNR $28.6 \mathrm{~dB}$ with WFC-GS

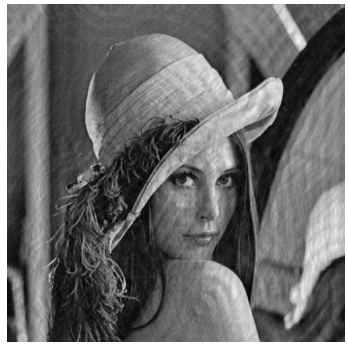

(d) PSNR 26.7dB with WFC-RAAR

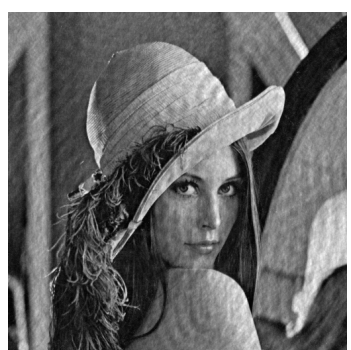

(b) PSNR 29.4dB with WFC-HIO

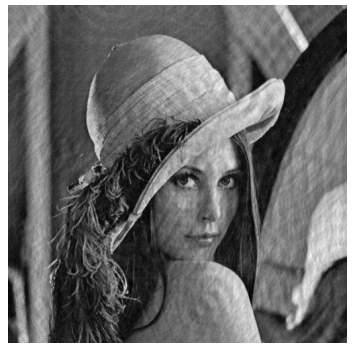

(e) PSNR 29.6dB with WFC-HAAR

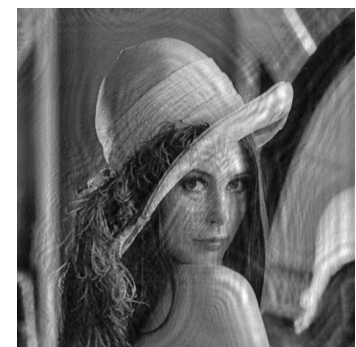

(c) PSNR 26.1 dB with WFC-AP

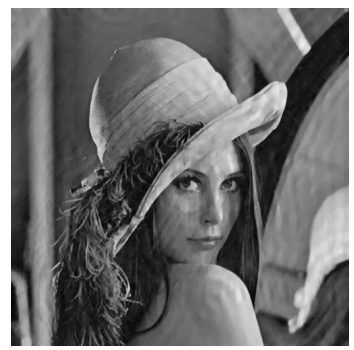

(f) PSNR $30.6 \mathrm{~dB}$ with WFC-FISTA

Figure 4.10. Comparison at iteration 7 of restoration quality between different WFC algorithms.

is shown in Figure 4.12. A defocused image is restored with a fixed low number of iterations. A crop of the restored image is magnified to highlight the differences in restoration for these small structures. We choose the eye as the detail for our observations using the same image as in the previous experiment. In the results, we see that the overall difference in restoration quality in small scale details is low. The restored images of all algorithms except for the WFC-FISTA look very similar. A difference can be seen in the amount of noisiness, as the result of WFC-AP is slightly more noisy than for example the result of WFC-RAAR, and a minor difference in the clarity of the eye lids. The WFC-FISTA result is much less noisy and still preserving fine details. The regularization property of the $L_{1}$ prior can be 
4. Applications and Results

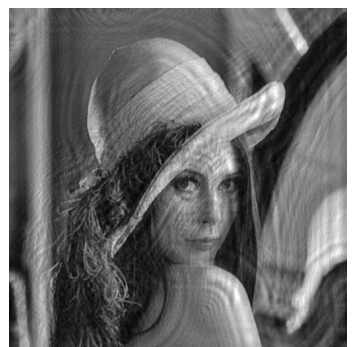

(a) PSNR 26dB with 3 it- (b) erations of WFC-HAAR

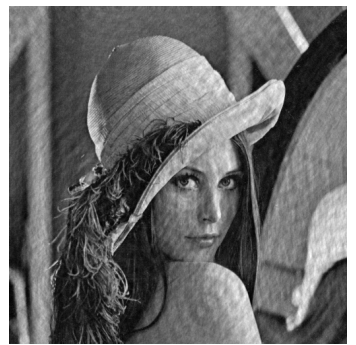

(d) PSNR $33 d B$ with (e) 25 iterations of WFCHAAR

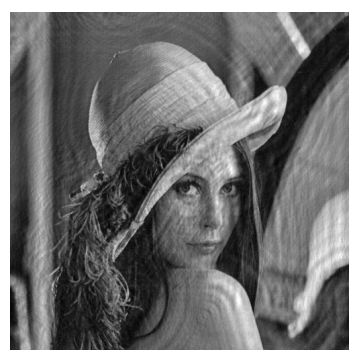

(b) PSNR 28dB with 5 it- (c) erations of WFC-HAAR

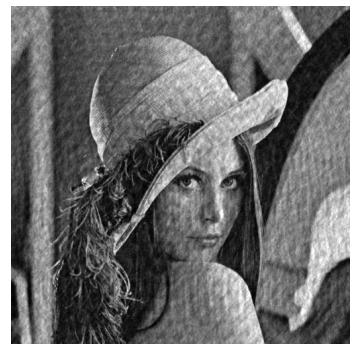

(e) PSNR $40 d B$ with (f) PSNR $47 d B$ with 100 iterations of WFC- 500 iterations of WFCHAAR

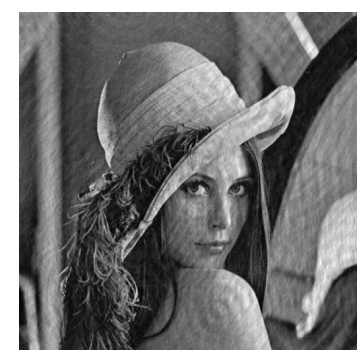

(c) PSNR $31 d B$ with 15 iterations of WFCHAAR

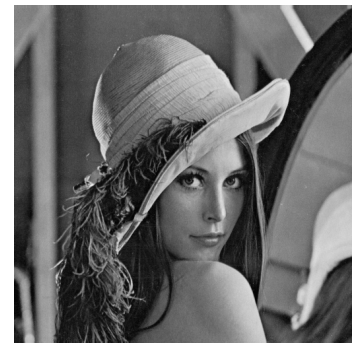

HAAR

Figure 4.11. Restorations with limited iterations. Results after 3, 5, 15, 25, 100 and 500 iterations are shown. For the input image see Figure 4.8b.

clearly seen. Partial over-smoothing can only be seen in direct comparison with the reference image.

In summary, the previous result is still confirmed, the WFC-FISTA algorithm shows a slightly better restoration and the WFC-AP algorithm a slightly worse restoration.

Runtime In the previous experiment we compared the convergence speed of the different method by PSNR at a fixed iteration count. We observed a large difference in the number of iterations necessary to reach a given quality. In the following, we focus on another aspect, that is runtime. 


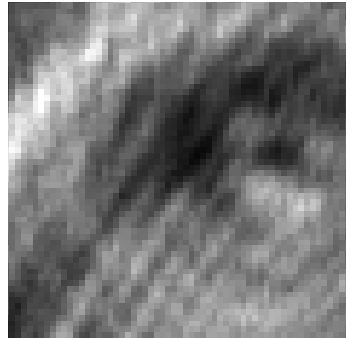

(a) Input image

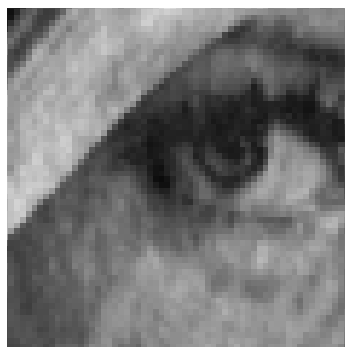

(d) WFC-AP

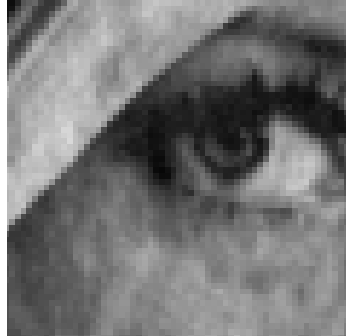

(b) WFC-GS

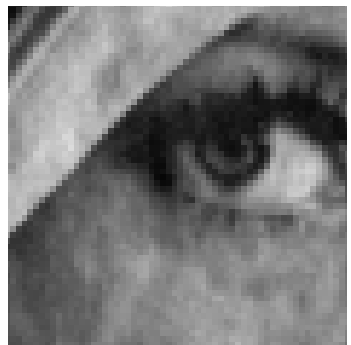

(e) WFC-RAAR

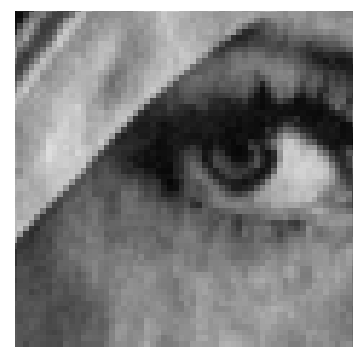

(c) WFC-HIO

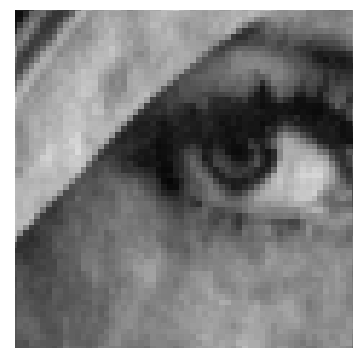

(f) WFC-HAAR

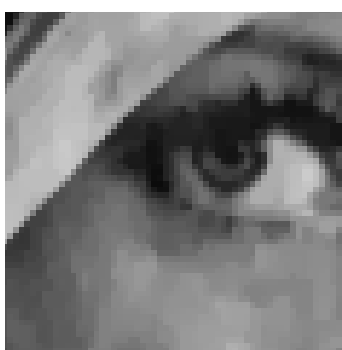

(g) WFC-FISTA

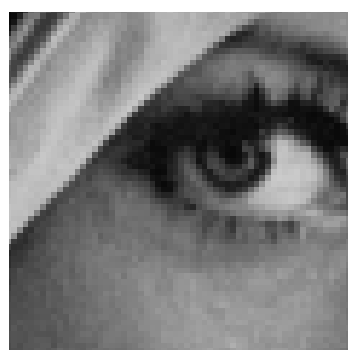

(h) Reference

Figure 4.12. Enlarged image detail for a visual comparison of the restoration with different algorithms. To highlight differences all images are restorations with only 10 iterations. 
The runtime is of course dependent on the number of iterations we use, but also on the steps they consist of and on the implementation we use and the hardware we use to run the experiments. As such, any result numbers are prone to a certain amount of subjectivity, which we aim to mitigate by documenting accurately, how the results were achieved.

For a detailed description of our implementation we refer to Section 4.1.

Of course, runtime depends strongly on the implementation and on the hardware. We developed a flexible multipurpose implementation, which allows us to conduct all the experiment in this section, however it is not overly optimized. The hardware platform for the runtime measurements is the same as specified in Section 4.1. We use a GPU based Matlab implementation of the projective WFC algorithms and a CPU based implementation for WFC-FISTA and measure the runtime on the same image with 500 iterations. The test image has $512 x 512$ pixels and has already been used in the previous Section for quality assessments. The results of the runtime measurements are shown in Figure 4.13. We can see that except for WFC-HAAR, the difference in runtime between the projective algorithms (WFC-GS, WFC-HIO, WFC-AP, WFC-RAAR) is low. WFC-HAAR is an exception, because with its Hageauzau operator it requires more intricate computations. The WFC-FISTA algorithm requires much more time for an iteration, 200ms per iteration, based on the CPU implementation. The algorithm with the longest runtime is the WFC-SS algorithm, because it needs to create a database of low-scale patches and perform lookups for every high-resolution image patch, it requires approx. 10s per iteration.

\subsubsection{Initialization}

In this section consequences of different initialization strategies are evaluated. The topic of initialization is important because of several factors. As wavefront correction is an optimization problem, the initialization should influence the convergence speed, because if we start close to the minimum only a low number of iteration steps is necessary to reach a certain quality of restoration. The second point is that wavefront restoration is a nonconvex problem, meaning that local minima can exists and a combination of a certain algorithm with the wrong initialization might become stuck in local minima. The third point that we have already observed in the 


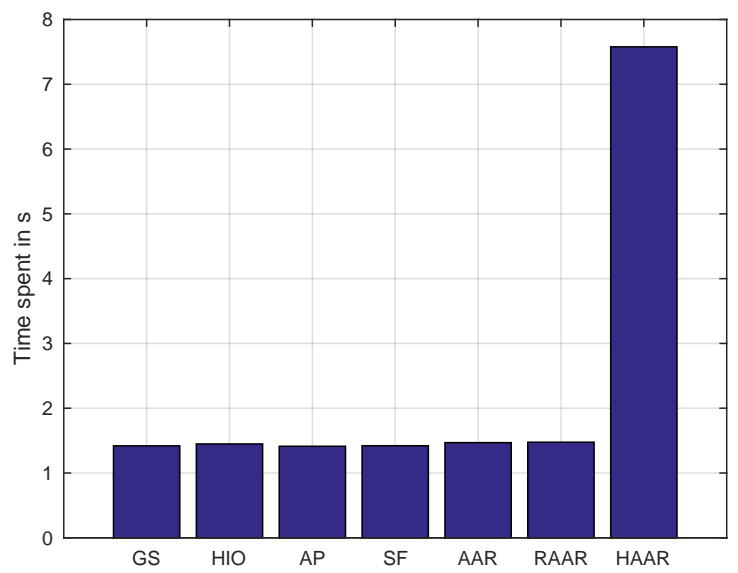

Figure 4.13. Speed comparison of the different WFC-algorithms in seconds per 500 iterations. The WFC prefix is omitted in the labels to save space. The WFC-SS algorithm is not included, because it does not fit the chart.

restoration of noisy images is that the projective algorithms do not converge to the true solution and the results get worse with more iterations (see WFC-AP in Figure Comparison of algorithm in restoration of noisy images).

Therefore, the consequences of choosing the initialization values must be analyzed. We evaluate the following choices of initialization:

1. ZI: Zero initialization sets the target image to zero

2. RI: Random initialization sets the target image to random complex values with a magnitude between 0 and 1 and phase between 0 and $2 \pi$.

3. MI: Initialization with the disturbed input image as amplitude and zero phase.

Figure 4.14 show a comparison of the restoration results of different initialization strategies and graphs with PSNR values per iteration are 
4. Applications and Results

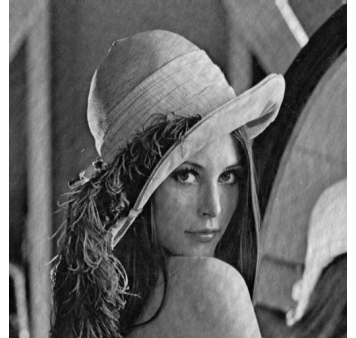

(a) WFC-GS with ZI

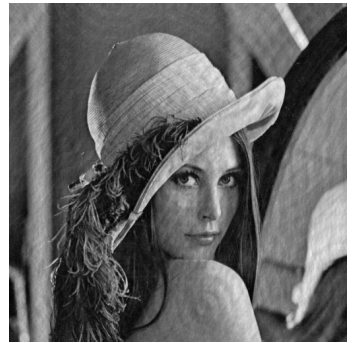

(d) WFC-AP with ZI

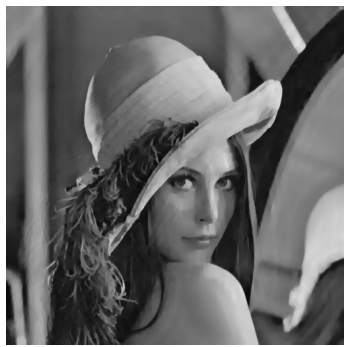

(g) WFC-FISTA with ZI

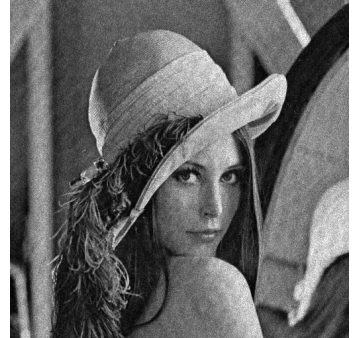

(b) WFC-GS with RI

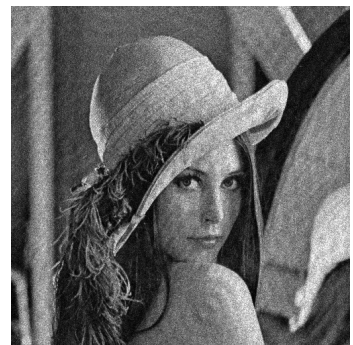

(e) WFC-AP with RI

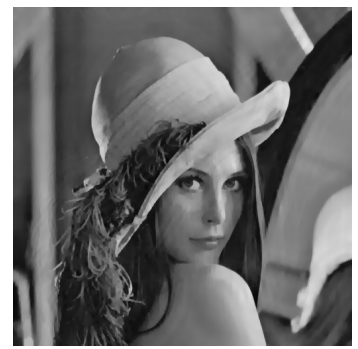

(h) WFC-FISTA with RI

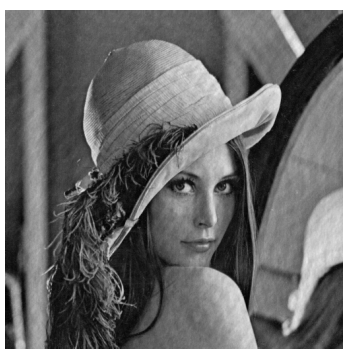

(c) WFC-GS with MI

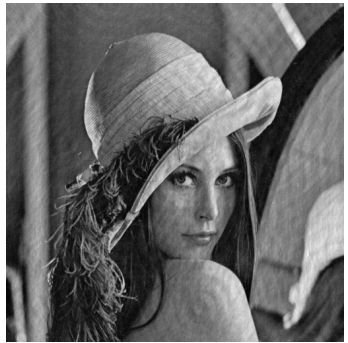

(f) WFC-AP with MI

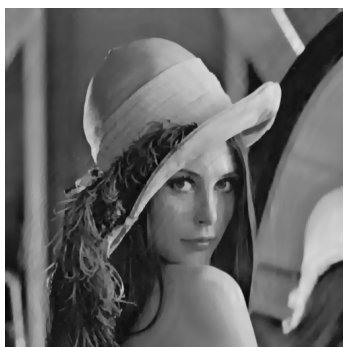

(i) WFC-FISTA with MI

Figure 4.14. Visual comparison initialization strategies. MI stands measured initialization, RI is random initialization and ZI stands for zero initialization, details see text. For input images, see Figure $4.8 \mathrm{~b}$. 
shown in Figure 4.15. We use the WFC-GS, WFC-AP and WFC-FISTA algorithms for this evaluation, to make sure be able to see if possible effects are algorithm dependent. These algoriths form a good cross section of the presented WFC algorithms, with the WFC-GS being the most intuitive method, the WFC-AP algorithm a projective approach, and WFC-FISTA a proximal algorithm.

In Figure 4.14 the results of different WFC algorithms with different initializations in iteration 200 are shown. We can clearly see that any of the wavefront correction methods WFC-GS, WFC-AP and WFC-FISTA shows good results with any initialization. The only difference visible is that the results with random initialization for WFC-AP and WFC-GS are noisier.

To analyze this phenomenon in more depth we need to calculate qualitative results with PSNR values. We measure the PSNR in comparison to the sharp reference image in every iteration and plot the result in Figure 4.15.

The previous results are confirmed. Most important is that all algorithms converge with all initialization strategies. This is a very good result and not unsurprising, because we have previously proven the nonconvexity of the wavefront correction problem. This means that even though the WFC problem itself and all projection algorithm except WFCHAAR are non-convex, non became stuck in local minima. The second result is that initializing with the disturbed input image magnitude (MI) is the best solution for the tested algorithms with the zero phase and magnitude initialization strategy showing almost identical results. Hence, the initialization with the magnitude of the defocused or otherwise disturbed image and zero phase is the initialization choice used for any other restorations in this thesis.

\subsubsection{Imperfect Input}

To simulate the effects of real data we conduct two tests: First, restoring an image with a spherical wavefront that deviates from the true deformation by $2 \%$ in radius. Second, we add Gaussian noise of different strength to the image, after restoration the result is still compared to the original noise free image.

The results for both tests (Figure 4.16 and 4.18 ) are very similar. Espe- 
4. Applications and Results

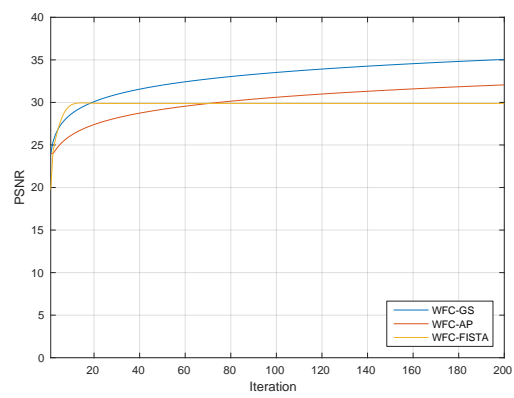

(a) MI: Initialization with disturbed image

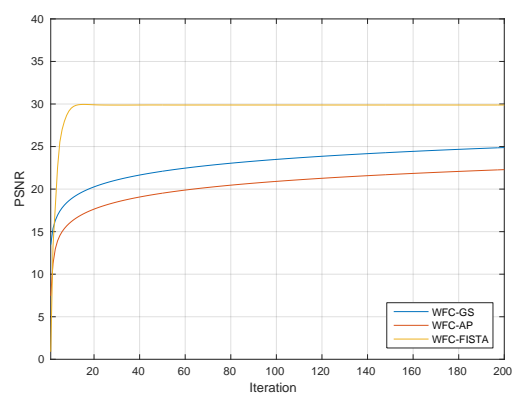

(b) RI: Random initialization

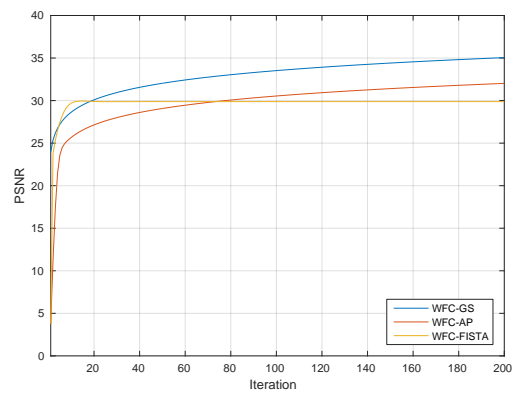

(c) ZI: Zero initialization

Figure 4.15. Comparison of different initialization strategies by their convergence. Show is the PSNR ratio per iteration against the undisturbed image. 
4.2. Results on Simulated Images

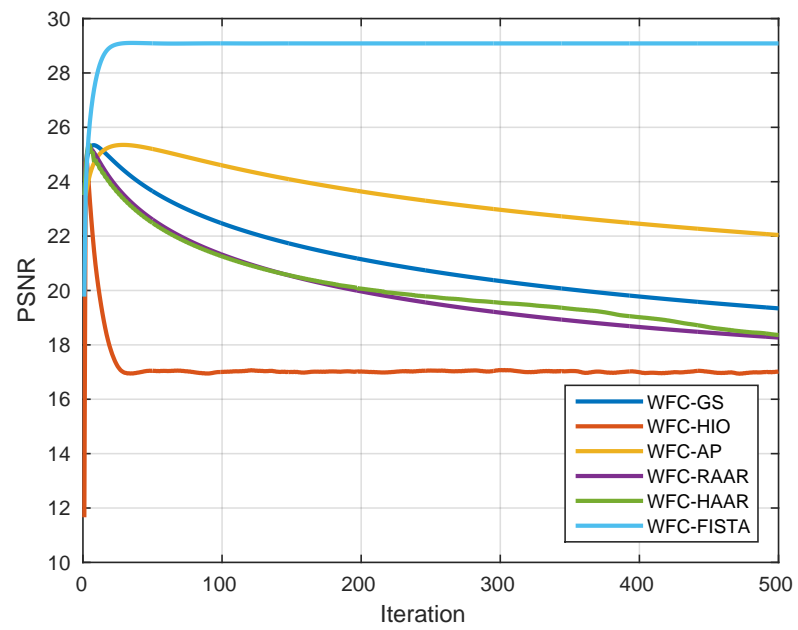

Figure 4.16. Restoration quality comparison for a given number of iterations by PSNR for different algorithms with a slightly wrong corrective wavefront with $2 \%$ radius.

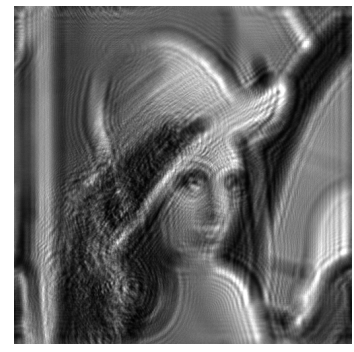

(a) No noise

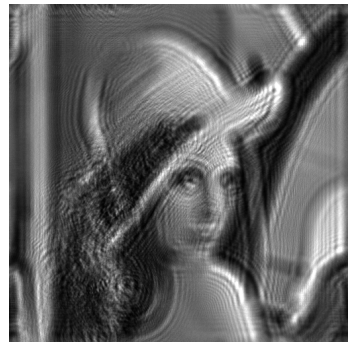

(b) $50 \mathrm{~dB}$ PSNR

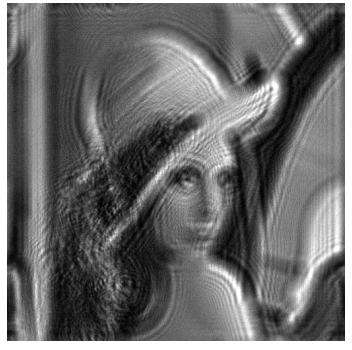

(c) $25 d B$ PSNR

Figure 4.17. Defocused image with Gaussian noise. 
4. Applications and Results

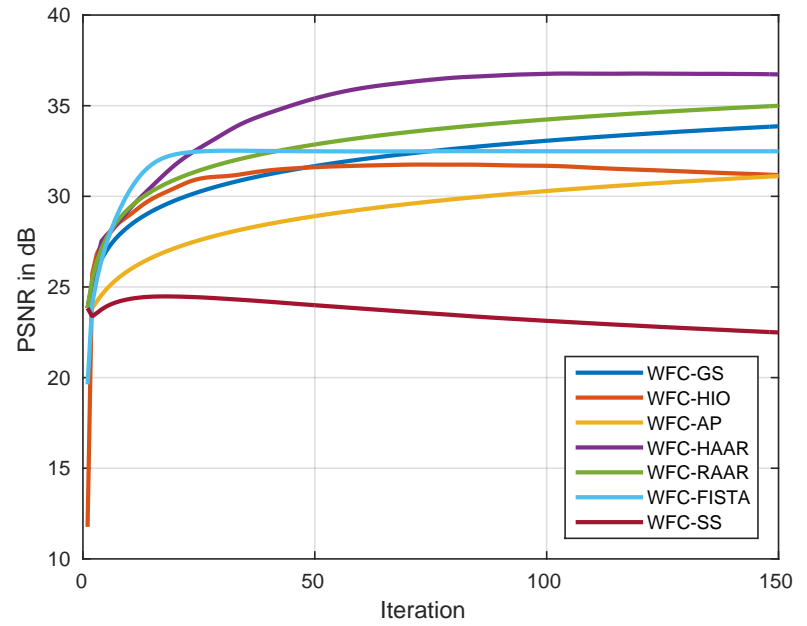

(a) Weak noise, 50dB PSNR

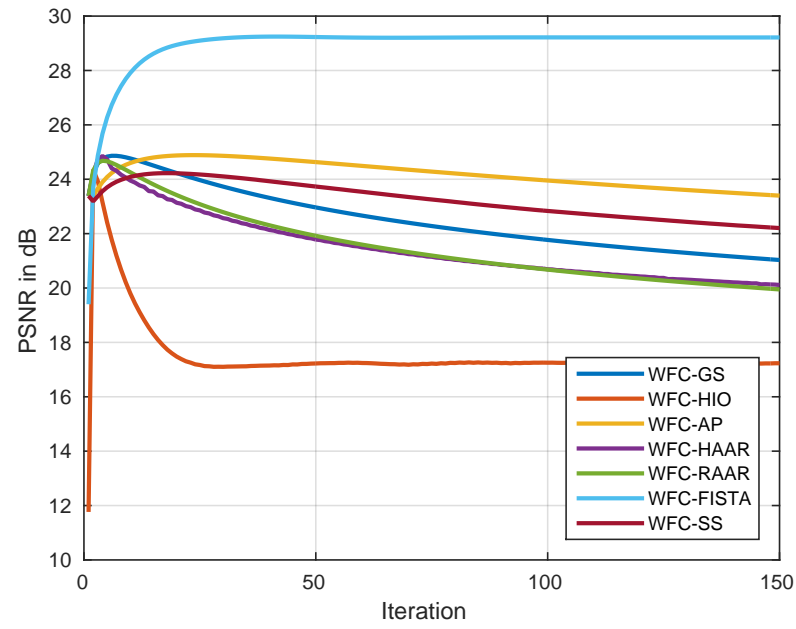

(b) Stronger noise, $25 d B$ PSNR

Figure 4.18. Comparison of algorithms in restoration of noisy images. 
cially, the graph with $25 d B$ noise shows strong similarity with the wrong wavefront graph. This confirms that with our approach the actual robustness of the algorithms can be measured.

The diagrams show a large difference in how well the algorithm are able to restore imperfect data. For low noise levels, the projective algorithms can be used with good results. We will discuss the results of WFC-SS algorithm separately. On the $50 \mathrm{~dB}$ noisy input data, we see a clear advantage in robustness for the WFC-RAAR algorithm over the WFC-HIO algorithms that was not present in noise free data 4.9.

In fact, the highest noise sensitivity has the WFC-HIO algorithm and the slowest algorithm (WFC-AP) is the most robust of the projective algorithms. The fastest convergence has the WFC-HAAR algorithm, however it is more complex to implement.

For noisy input data or in case the optical aberrations are not precisely known, the WFC-FISTA is superior over the projective algorithms due to the noise canceling property of the total variation regularizer. With a $25 d B$ noise input image, the restoration result of the WFC-FISTA reaches a PSNR of $29.2 d B$. This means that the result image is a nearly accurate representation and less noisy than the input data.

Self-Similarity Wavefront Correction The WFC-SS algorithm has to be discussed separately. We see that for weak noise (see Figure $4.17 \mathrm{~b}$ the results are not good, as it shows the worst results of any algorithm tested. Because the algorithm has a strong similarity to the WFC-AP algorithm, we interpret this result as an overly strong regularization. Nevertheless on the graph with stronger noise $4.17 \mathrm{c}$, the result are comparable with the weaker other methods, such as the WFC-AP and WFC-GS algorithm. With stronger noise the WFC-GS algorithm shows worse results.

We want to analyze the behavior of this algorithm in more detail on images with even stronger noise and apply the most noise resistant methods from the previous experiment, the WFC-AP and WFC-FISTA algorithm, together with the WFC-SS algorithm on an image with noise level $10 d B$ PSNR. The results are shown in Figure 4.19. We see a very noisy input image and the restoration with the respective algorithm. The result from WFC-AP seems to have increased the noise in the image, while it seems like a restored image has been layered in the background. The 
4. Applications and Results

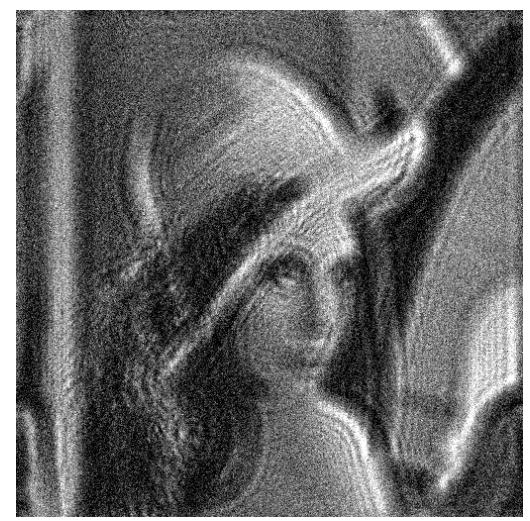

(a) Input with very strong noise of 10dB PSNR

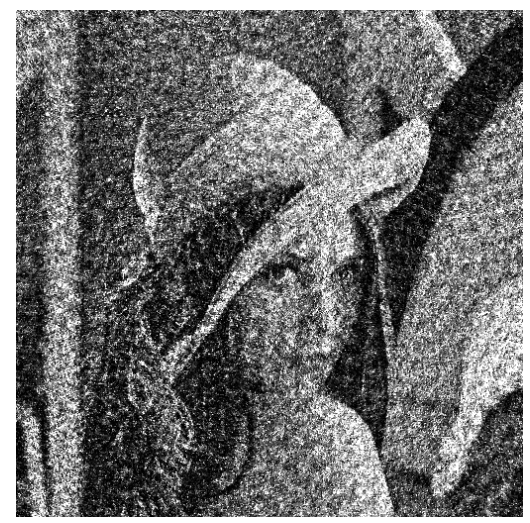

(c) Restored with WFC-FISTA

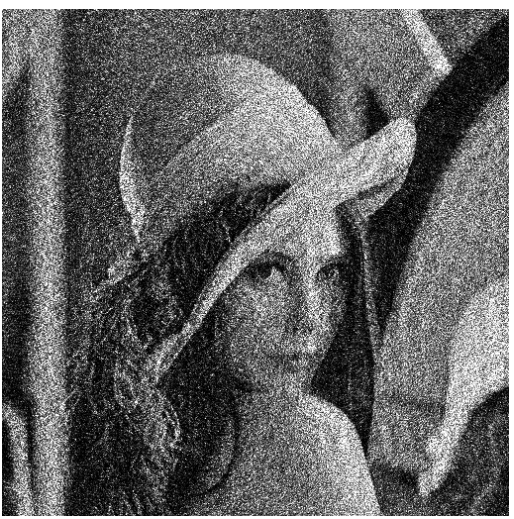

(b) Restored with WFC-AP

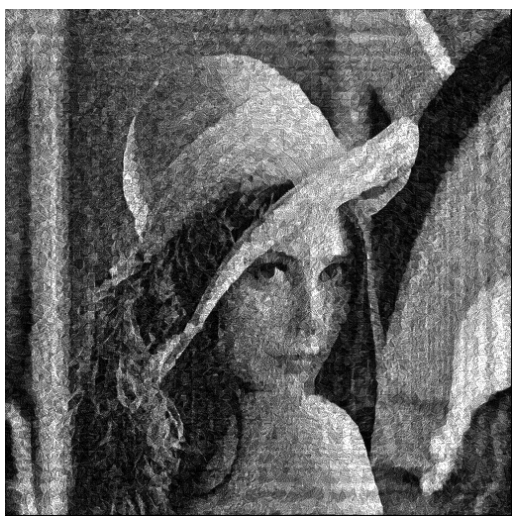

(d) Restored with WFC-SS

Figure 4.19. Comparison of restoration with very strong noise. Wavefront correction with self-similarity prior can cope with this level of noise. 


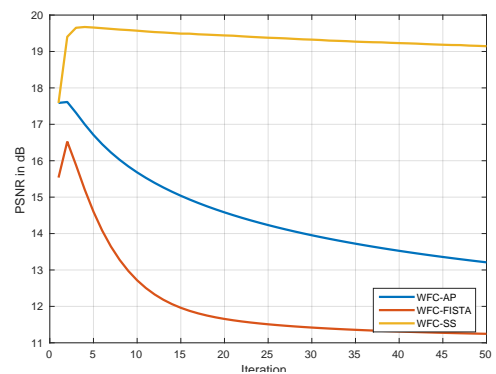

(a) Noisy input with $10 d B$ PSNR

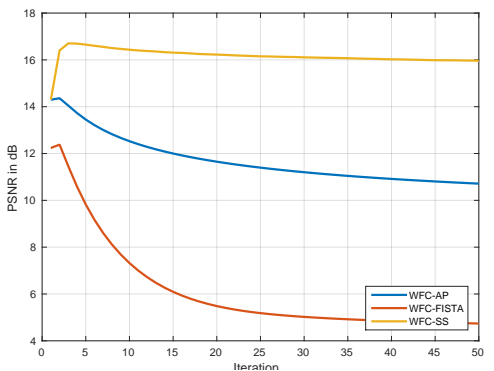

(b) Noisy input with $5 d B$ PSNR

Figure 4.20. Comparing the results of the best algorithm for weaker noise WFC-AP and WFC-FISTA with WFC-SS for strong noise.

WFC-FISTA algorithm produces granular noisy artifacts, which make the results visually even worse than the WFC-AP algorithm result. A usable result is produced only with the WFC-SS, but results get worse with more iterations.

While we accept that the PSNR is not the ultimate measure of visual quality in cases of strong noise, a qualitative measurement is necessary. We set up a similar experiment to the previous restoration quality graphs, but only with these methods and much stronger noise. The input images have only a PSNR of 10 and 5. The graphs are plotted in Figure 4.20. The curves of the WFC-AP and WFC-FISTA confirm the results of our visual comparison, that these algorithms are not suitable for such noisy inputs. Thus, the WFC-SS algorithm is the only WFC algorithm that can handle noise at this level.

Another observation is that in any of the shown graphs the PSNR values of the restoration with the WFC-SS algorithm becomes worse with increasing iterations, less than 5 iterations seem to be ideal. Self-similarity introduces a strong prior that is strongly denoising and is particularly suited for recovering sharp edges. However, a repeated application of the self-similarity prior seems to remove the structure or texture available in the prior iterations and has as shown detrimental effects. Moreover, selfsimilarity prior also increases the boundary ringing artifacts and cannot distinguish them from the image signal, because these are large structures 
looking like edges. This phenomenon can be seen in Figure 4.19 in the outer part of the WFC-SS restoration.

\subsubsection{Results of Large Image Processing}

In this section we present the results for large image processing technique discussed in Section 3.8.

To be able to process very large images we switch to the CPU implementation of the algorithm in this section. For the fast Fourier transform we rely on Matlab version 2015b [Mat15], which itself uses the Fastest Fourier Transform in the West (FFTW) library [FJ98] [FJ12] This library uses a collection of Fourier transform algorithms and selects the fastest for a given input configuration. This allows us to assume that the fftw implementation is optimized for arbitrary input size, which is supported by publicly available benchmarks [FJ04].

For our implementation we use 64 tiles arranges as 8 per row and 8 per column. The result is shown in Figure 4.21, where the original image, the input image with simulated defocus, a direct restoration and the restoration with tiling is compared. The tiling restoration also performs well and produces a sharp restoration, however ringing artifacts at the boundaries of every tile are visible.

The runtime for processing an image with 6000 pixels width and 6000 pixel height took 30.8 second without tiling and 15.5 second with the tiling WFC algorithm. Hence. using the tiling WFC the runtime of the algorithms for large images can be significantly reduced. Another advantage is the drastically lowered memory demand, which is important for large images. For example an image of size of size $8000 \times 8000$, cannot be processed on a machine with $16 G B$ memory, because although the image fits into memory itself, the memory demands of the entire algorithm lead to a crash of the Matlab kernel. For GPU based processing this is even more relevant as graphics cards with over $4 G B$ of memory are currently (2017) costly. This means our tiling based strategy can be even faster, because the image can be processed on a fast GPU, which usually has smaller memory.

\footnotetext{
${ }^{7}$ Rembrandt Harmenszoon van Rijn ('Rembrandt'), The Company of Frans Banning Cocq and Willem van Ruytenburgh, known as the 'Night Watch' [Public domain], via Wikimedia Commons from Wikimedia Commons https://commons . wikimedia.org/wiki/File\%3ARembrandt_van_
} 


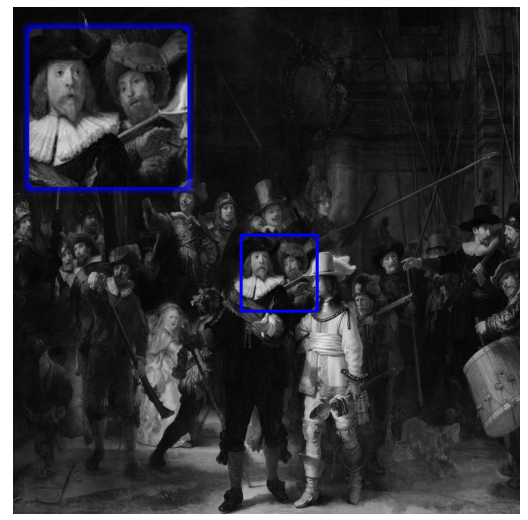

(a) Original image ${ }^{7}$

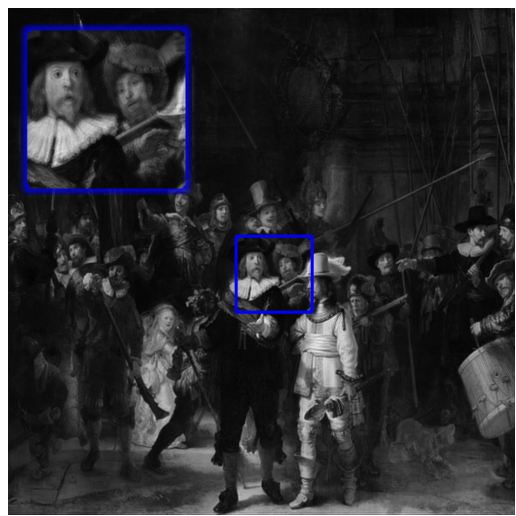

(c) Direct restoration, $30.8 \mathrm{~s}$

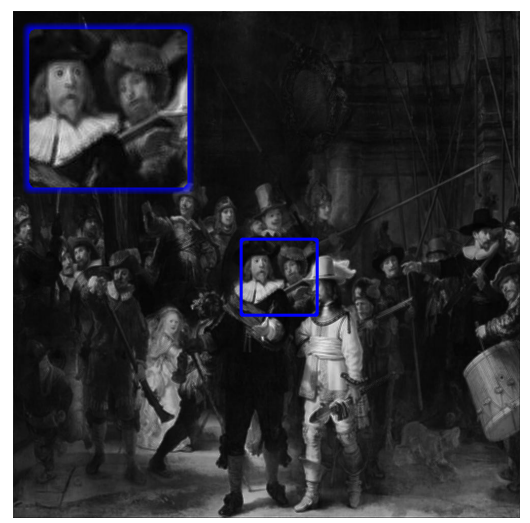

(b) Input image

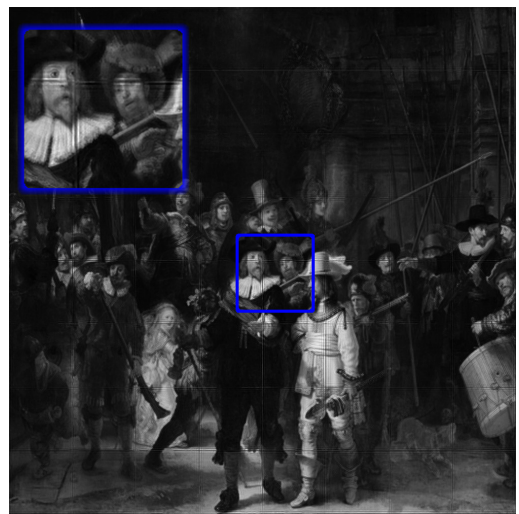

(d) Tiling restoration, $15.5 \mathrm{~s}$

Figure 4.21. Comparison of result with tiling and non-tiling CPU WFC-AP algorithm restorations. The sharpness of the restoration can be seen best on the eye pupils of the left person in the magnification. The tiling approach exhibits boundary artifacts on the tile borders. 


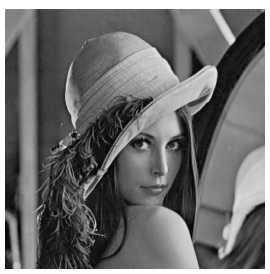

(a) Ground truth image

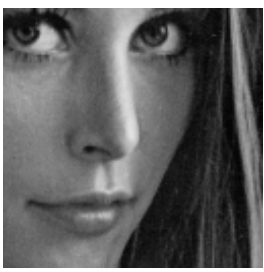

(b) Inner crop of (c) Image with sim-(d) the ground truth ulated blur and vis- the blurred image image
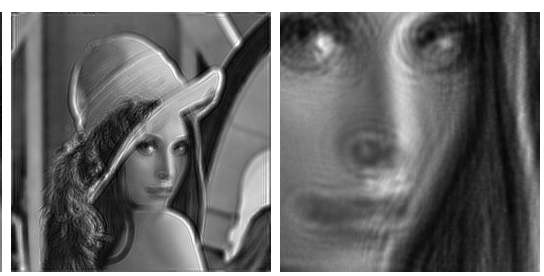

(d) Inner crop of ible boundary ring-without boundary ing ringing

Figure 4.22. Ground truth and input for boundary ringing evaluation.

\subsubsection{Comparison of Boundary Ringing Suppression Tech- niques}

Boundary ringing can strongly influence the results of the WFC algorithm. Thus, we discuss different approaches for the suppression of boundary ringing in Section 3.9.

In this section we want to evaluate the different approaches and find out which one works best for wavefront correction. To be able to compare the results against a common ground truth this experiment requires an image with simulated aberration. Very important for this evaluation is that the input image does not contain any boundary ringing. If we apply simulated defocus on an image with the technique discussed previously, clearly boundary ringing occurs and a restoration can only remove it if the image is not cropped or scaled in any way, as it relies on the same boundary effects to compensate them. Hence, we use only the inner-most crop of the image with simulated defocus to remove the ringing artifacts (Figure 4.22c and Figure 4.22d). For the ground truth image we apply the same image crop (Figure 4.22a and Figure 4.22b). A positive side effect of this procedure is that the image size is reduced and thus potential boundary ringing in the restoration becomes more strongly visible. $\mathrm{s}$ For this evaluation, we compare the results of 50 iterations of WFC-AP

Rijn-De_Nachtwacht-1642.jpg 


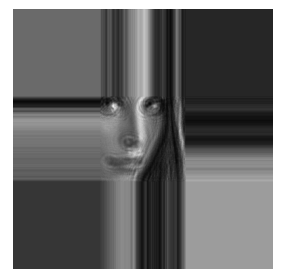

(a) Replicate

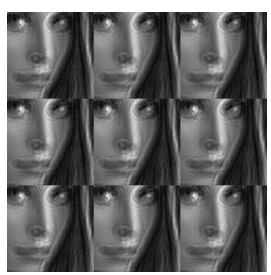

(b) Circular

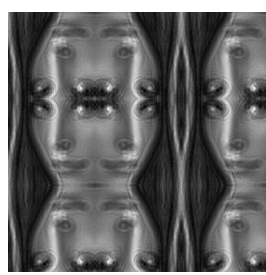

(c) Symmetric

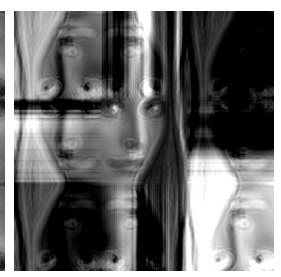

(d) Antisymmetric

Figure 4.23. Input images for different padding strategies after half image width padding is applied.

on an test image with $128 \times 128$ with different techniques for boundary suppression, which are motivated and explained in Section 3.9. As a baseline, the first test is directly applying the algorithm on the test image. Secondly, edge tapering is tested with a Gaussian kernel with width and height of 15 pixel and $\sigma=5$. Then we apply edge padding with the replicate circular symmetric and antisymmetric techniques. For padding, there is an option. We can either pad the image with an image width of pixels, motivated by the thought that this enforces a stronger periodicity in the image, or use smaller padding with a half image width and see whether this is sufficient.

The second option we test is whether it is better to apply the WFC algorithm with its application of constraint on the entire image or whether it is better to apply the constraints only on the core image without padding. For this test we modified our WFC implementation in such a way that the entire image is transformed in each step, but constraints are only applied on the inner image. This technique has similarities to the support constraints of the Fienup phase retrieval algorithms, see [Fie82] and Section 2.3. In this context, we call this technique partial application of constraints.

The quantitative results for all these ringing suppression techniques are listed in Table 4.1. For a qualitative comparison and as a visual guide to the PSNR numbers, the result images of selected techniques are shown in Figure 4.24. Our first observation from these results is that there are large differences between the different methods. We see the importance of 


\section{Applications and Results}

Table 4.1. Restoration quality measurement by peak signal to noise ratio (PSNR) in $\mathrm{dB}$ of different boundary ringing suppression techniques. The algorithm used is WFC-AP with 50 iterations.

\begin{tabular}{cccc}
\hline Method & Constraints & Padding Size & PSNR \\
\hline None & Full & & 22.185 \\
\hline Edge tapering & Full & & 24.5587 \\
\hline Replicate & Full & Full width & 28.4445 \\
Symmetric & Full & Full width & 27.0632 \\
Antisymmetric & Full & Full width & 24.6593 \\
Circular & Full & Full width & 22.3028 \\
\hline Replicate & Full & Half width & 28.0084 \\
Symmetric & Full & Half width & 27.0727 \\
Antisymmetric & Full & Half width & 24.1152 \\
Circular & Full & Half width & 22.2877 \\
\hline Replicate & Partial & Full width & 29.1109 \\
Symmetric & Partial & Full width & 28.8509 \\
Antisymmetric & Partial & Full width & 28.8494 \\
Circular & Partial & Full width & 27.5655 \\
\hline Replicate & Partial & Half width & 29.0637 \\
Symmetric & Partial & Half width & 28.8045 \\
Antisymmetric & Partial & Half width & 28.8018 \\
Circular & Partial & Half width & 27.5346 \\
\hline
\end{tabular}

an evaluation encompassing the different padding strategies and sizes. It is unexpected that the less sophisticated approach of replication padding yields better result than any other method. Our next result is that full width padding always results in less ringing than half width, which is to 

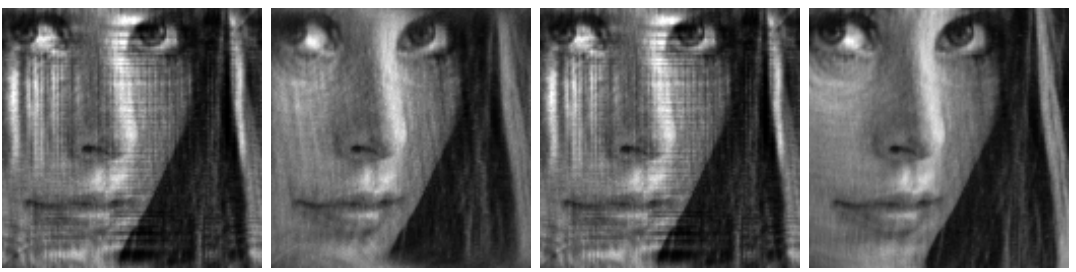

(a) No ring-(b) Edge tapering, (c) Circular with (d) Replicate with ing supression, PSNR: $24.5587 \mathrm{~dB}$. full constraint partial constraint PSNR: $22.185 \mathrm{~dB}$. enforcement and and full padding, full padding, PSNR: $29.1109 \mathrm{~dB}$. PSNR: $22.3028 \mathrm{~dB}$.

Figure 4.24. Visible difference of different boundary ringing suppression techniques. A large difference between all result images can be seen in the visibility of streaks on the left side of the image.

be expected. Between the padding methods a clear ranking, consistent over padding size and constraint application, can be made. The best results are achieved with the replication method, the second best with symmetric, then comes the antisymmetric, then the circular method.

The edge tapering method which relies on modifying the input image is still better than the worst padding method. Its strong point is that the image size is not enlarged and therefore the restoration is not slowed. Nevertheless we believe that the overall the difference in quality to the replicate method with partial constraint and full padding is too high. Another point to be made is the difference in scores with full and partial enforcement of the constraint. Although it can be tricky to implement, as boundaries must be observed in every step of the algorithm, the good results show that it is very useful.

\subsubsection{Application of Incoherent Deconvolution on Coher- ent Images}

In previous sections the novel coherent image restoration algorithm is applied on coherent images. The difference between coherent and incoherent imaging has been explained in more detail in the introduction. 
The main motivation of this thesis is that standard deconvolution algorithms cannot show useful results on coherent images, this led to the development of the presented novel class of coherent restoration algorithms. The reason is the difference in the image formation model, linear in amplitude or linear in intensity. In the following we shall verify, that the incoherent algorithms indeed do not restore coherent images. For this experiment defocus is simulated on an image. Thus, we have perfect conditions without noise. As examples of incoherent algorithms the classic, but slightly outdated Richardson-Lucy algorithm and a more modern algorithm, the 'fast image deconvolution using hyper-laplacian priors' algorithm by Krishnan and Fergus [KF09] are applied on this coherent image, shown in Figure 4.25. This necessary PSF for these non-blind deconvolution algorithms is obtained by applying the Fourier transform on the wavefront deformation.

The results of the Richardson-Lucy algorithm and a modern deconvolution algorithm by Krishnan and Fergus [KF09] are shown in Figure $4.25 \mathrm{c}$. They are visually not pleasing and show a completely failed restoration. Hence, they confirm that on images with such strong aberrations incoherent deconvolution algorithms are not applicable. This is because of the theoretical ill-fit of these methods. They cannot consider coherent phenomena such as interference.

These clear results confirm that standard deconvolution algorithms fail in application on coherent images. This confirmation validates the theoretical motivation in the introduction chapter and is thereby fundamental for the motivation of the novel algorithms.

\subsection{Microscopic Images}

\subsubsection{Experimental Setting}

To goal of this thesis is to not only develop novel algorithms, but also the test, evaluate and analyze them on simulated and even more important real images. Hence a realistic setup is necessary. After earlier experiments on an optical bench, we decided to use a KERN optics professional inverted microscope with custom LED illumination to be as close as possible to the 


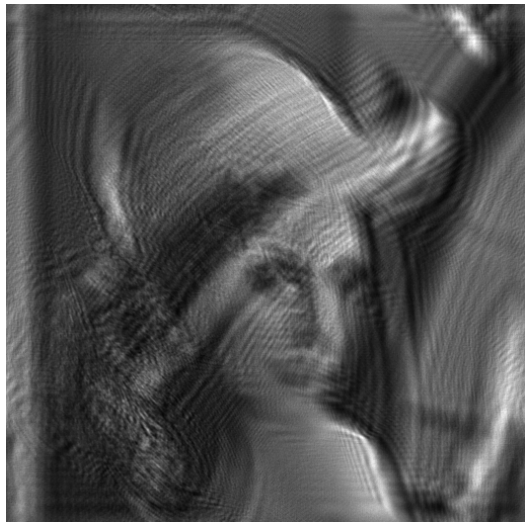

(a) Input

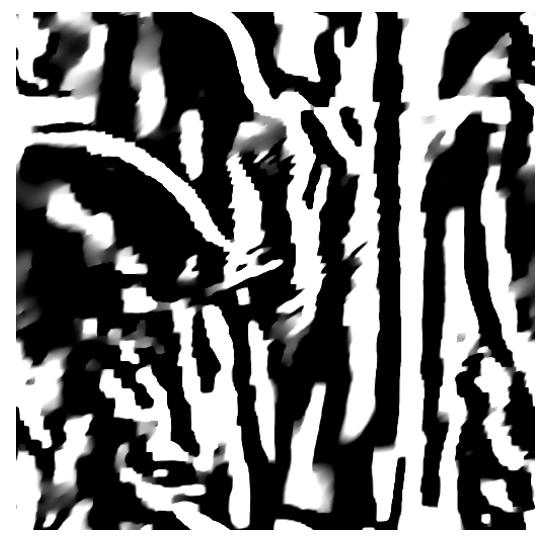

(c) Algorithm of Krishnan and Fergus [KF09]

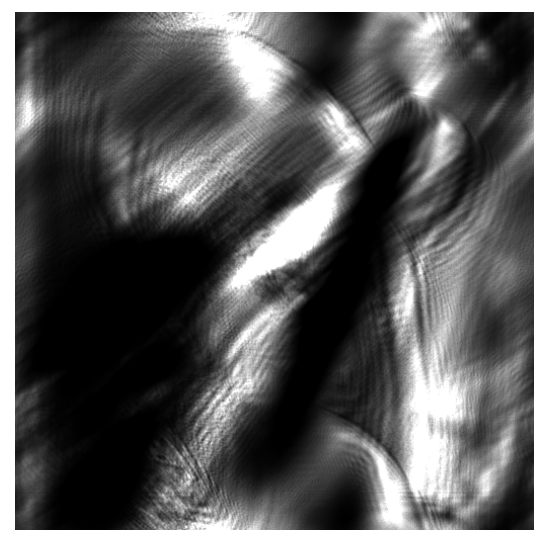

(b) Richardson-Lucy algorithm [Ric72] [Luc74], implementation see [Mat15]

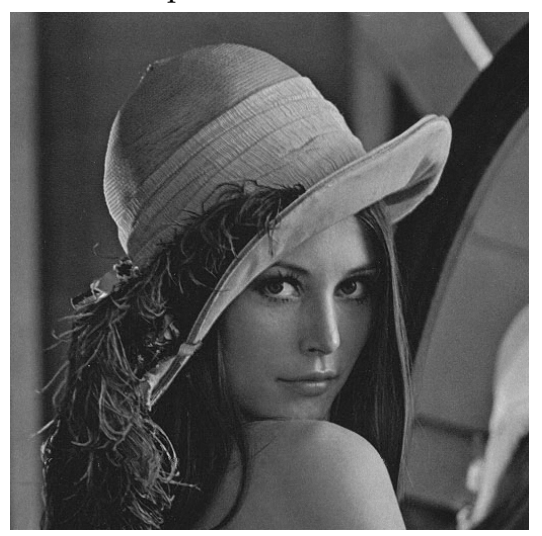

(d) Restoration with WFC-GS

Figure 4.25. Restoration results on Image 4.25a with incoherent deconvolution algorithms, Richardson-Lucy deconvolution [Ric72] [Luc74] and fast image deconvolution using hyper-laplacian priors [KF09]. Image $4.25 \mathrm{~d}$ show the result with the novel WFC algorithm. 


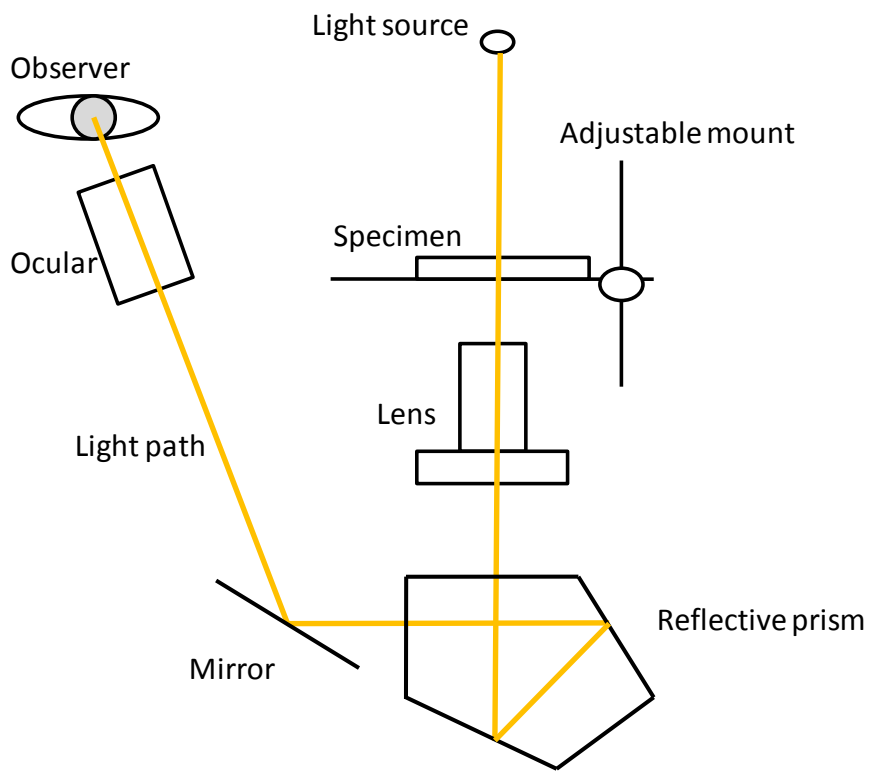

Figure 4.26. Schematic drawing of the optical path in microscopic application. ${ }^{1}$

real application in coherent microscopy. We believe that such a setup is crucial to verifying the correct working of the algorithm.

The inverted microscope has a slightly different light-path to noninverted microscopes, which allows us to easily apply our illumination. A schematic drawing of the light path is shown in Figure 4.26. The original light source of the microscope was replaced with an LED, which is shown in Figure 4.27a.

The microscopic image can be seen by the naked eye via an ocular, but for capturing, a digital camera is necessary. A photograph of the illumination mount into the microscope and with the attached Point Grey industrial camera for image acquisition is shown in Figure 4.27.

The imaging model behind WFC is physically based and as such

\footnotetext{
${ }^{1}$ drawing inspired by http://encyclopedia2. thefreedictionary.com/operating+microscope
} 


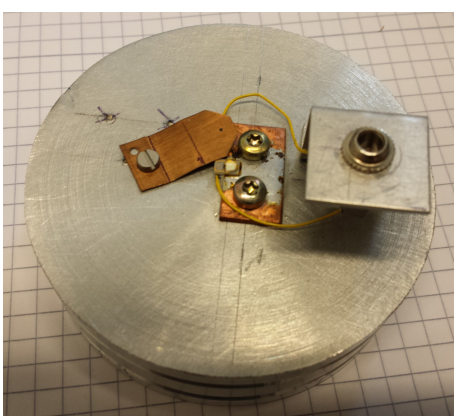

(a) Illumination unit, with LED and a small aperture moved aside for better visibility of the

LED.

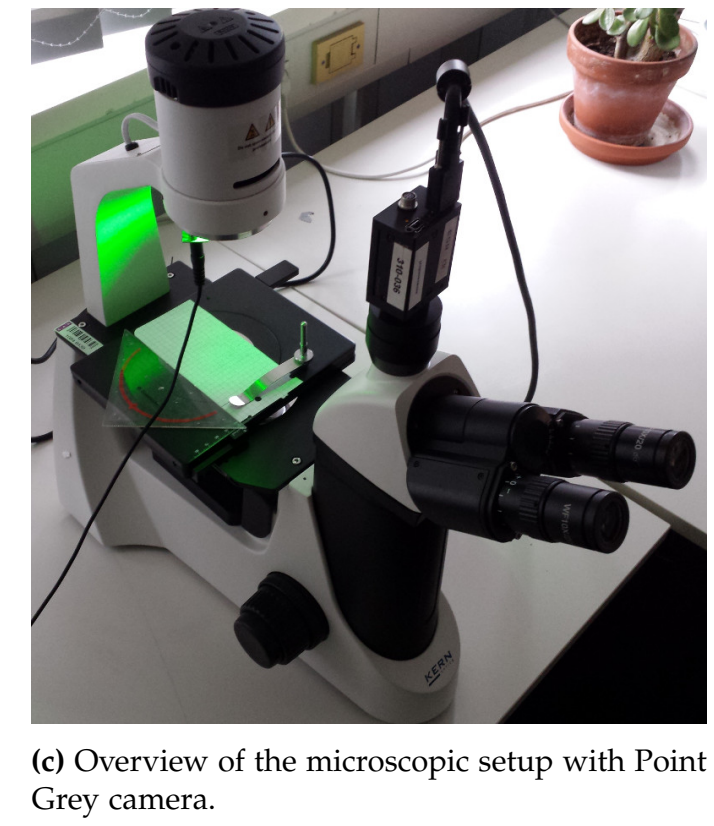

(c) Overview of the microscopic setup with Point Grey camera.

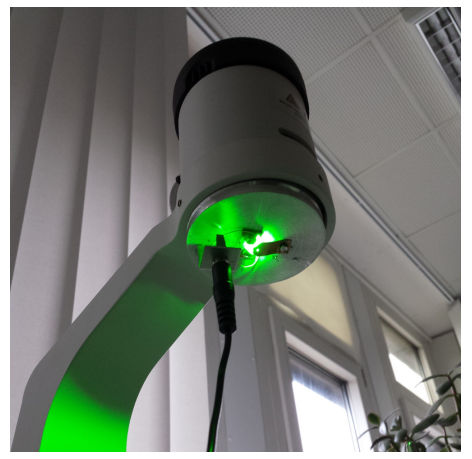

(b) Illumination mounted on the microscope.

Figure 4.27. Custom illumination and microscopic setting, a paper tube to reduce light scatter and environmental light is recommended. 
assumes zero offset and linearity with amplitude. In general consumer digital cameras apply a gamma curve with $\gamma=2.2$, industrial cameras can be configured for full linearity. We have experimented with two different camera-setups for the microscope. A Pentax Q10 consumer digital camera with c-mount adapter and the Point Grey Grasshopper 2 industrial camera. The images captured with a Pentax Q10 require offset removal and special RAW file conversion to obtain a linear image. The offset level in some cases must be extracted directly from the RAW file property nodes or it must be measured by taking a dark shot. Thus using this camera is a bit tedious. In contrast the images of the Point Grey Grasshopper 2 camera are linear and do not require any black level compensation. To lower image noise, setting a high sensor integration time (low shutter speed) is recommended. The images captured record the light intensity distribution across the image sensor. Because the algorithms work on amplitude, the square root of the measured values is calculated as a first step.

\subsubsection{Results}

As a first step, our computational model is verified. Figure 4.28 verifies the validity of the simulated results by comparing a measured aberrated test target with the result of simulated aberrations. This means that the mathematical model we use to model aberrations and blur is valid and our simulated results are relevant.

In Figure 4.29 a strongly defocused measured image of a USAF test target is restored with the novel algorithms. Because it is difficult to calculate PSNR values on simulated images, we use qualitative comparison of visual quality for natural image. From Image 4.29c obtained with the basic WFC-GS algorithm a slight improvement with less noise in the restoration can be seen in the result of WFC-AP in Figure $4.29 \mathrm{~d}$, the result of WFC-HAAR seems to be similarly noisy. In contrast, a large improvement can be seen with the WFC-FISTA algorithm. This confirms the results on simulated images. The regularization removes the restoration artifacts while the image content is preserved.

With the next experiments, we want to explore the limitation and capabilities of the algorithm on real images acquired with a microscope and led illumination. Small objects such as the small blood vessels streaks 


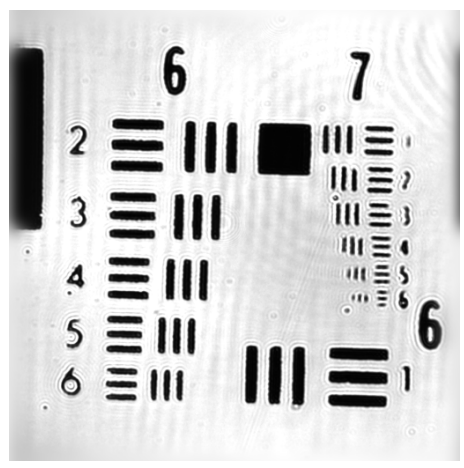

(a) Measured image at focus

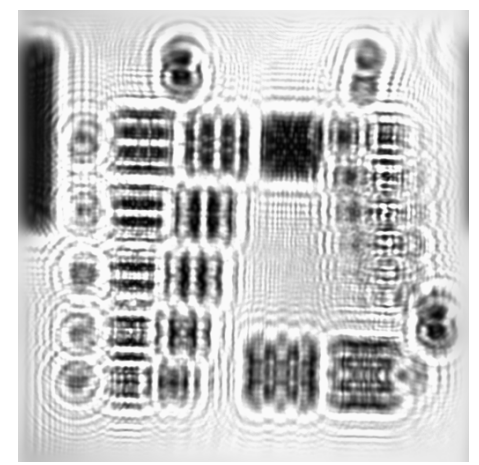

(c) Measured intensity distribution at $1.95 \mathrm{~mm}$ from sharp focus image (a)

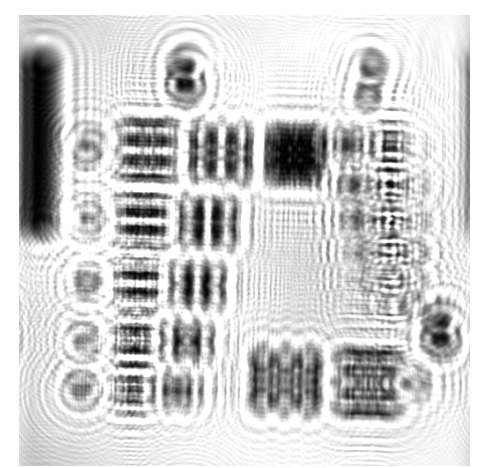

(b) Synthetic defocus on (a), same strength of defocus used as for the restoration of image (c)

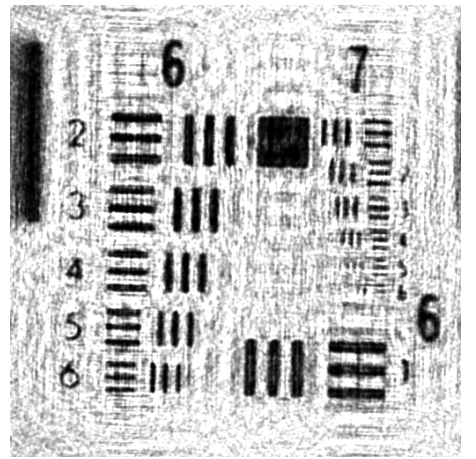

(d) Restored image from (c) with 75 WFC-GS iterations

Figure 4.28. Comparison of the sharp original, the measured defocused image, the synthetic defocus image and the restored image. 
4. Applications and Results

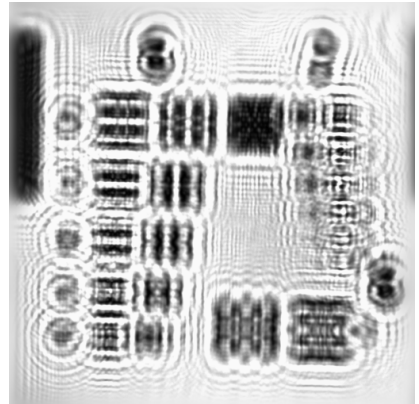

(a) Input

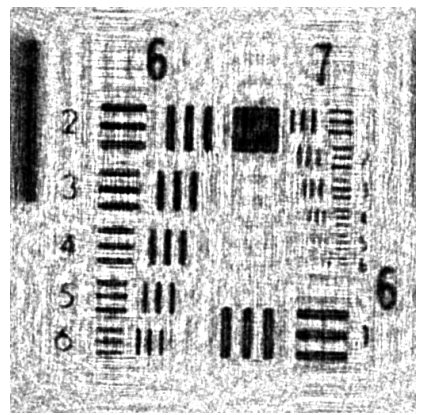

(c) WFC-GS

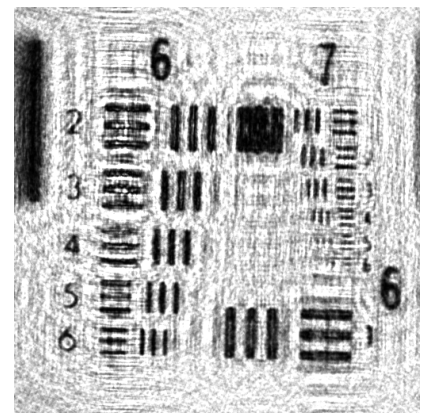

(e) WFC-HAAR

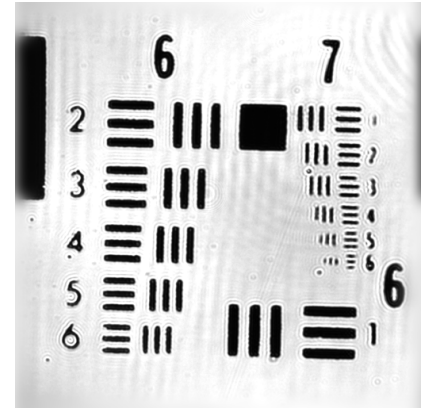

(b) Reference image with different imaging setting

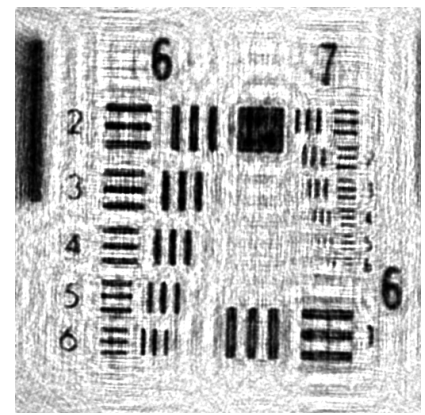

(d) WFC-AP

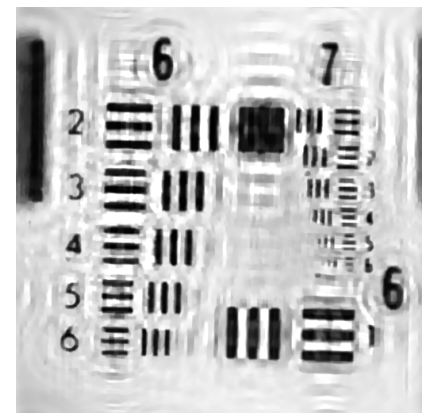

(f) WFC-FISTA

Figure 4.29. Comparison of different novel algorithms on a microscopic image of a test target. 
in Image 4.30 are not visible anymore in a strongly defocused image. Only the ringing artifacts hint to their existence. They can still be restored, because the information about the objects structure is preserved in these ringing artifacts, see Figure 4.30. The restoration also shows the advantages of the novel WFC-FISTA algorithm over the prior WFC-GS and the more stable projective algorithm WFC-AP.

Cell counting is an important task in medical imaging. In Figure 4.31 a pigeon blood smear is shown. It is very hard to make an accurate cell count without guessing on the input image, while with the restoration even the shape of the blood cells can be measured accurately.

An example of the limits of the WFC-FISTA algorithm is the restoration of a human brain tissue in Figure 4.32. The image shows a seemingly noisy input image and we can see how the restoration image quality is limited by it. Even with longer exposure times, we were unable to remove this unsteady background, which is not formed by image sensor noise. We reason that this is due to the light interference in a three dimensional non-homogeneous medium. The black dots on the sample are cores of transparent blood cells. This is not an ideal imaging situation for wavefront correction. Another point is that even though the cores of the surrounding cells and the structure of the neuron are clearly sharper in the right image, some parts of the neuron remain unsharp. From the three dimensional structure of the neuron only one layer can be focused using the WFC algorithm. A completely sharp image would require a spatially variant defocus estimate.

\subsection{Focused Shadowgraph}

In the DFG (german research foundation) funded project "All-in-focus: Combining lightfields and shadowgraphs for advanced underwater imaging" in cooperation with GEOMAR Helmholtz Center for Ocean Research Kiel a novel kind of sensor to gather information about the abundance and specifics of organic matter in the water column is developed. Special focus is laid on small sized matter in low abundance and its identification in a large observed volume. The marine-biological motivation behind this project is to explore the global transport of maritime biomass and plankton. 
4. Applications and Results

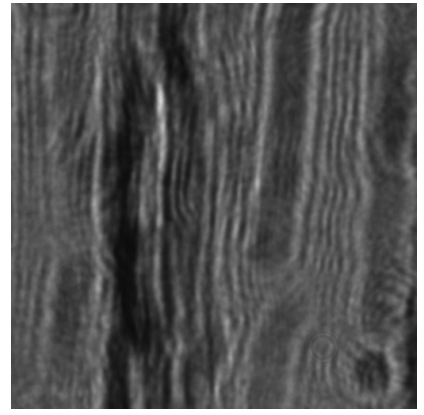

(a) Input

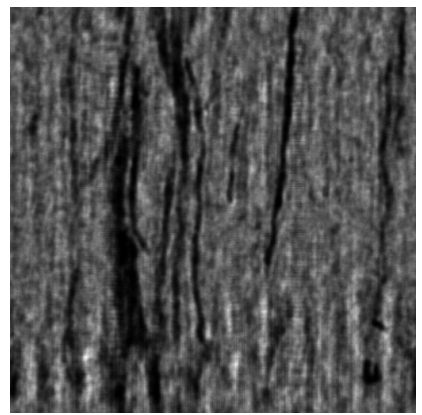

(c) WFC-GS

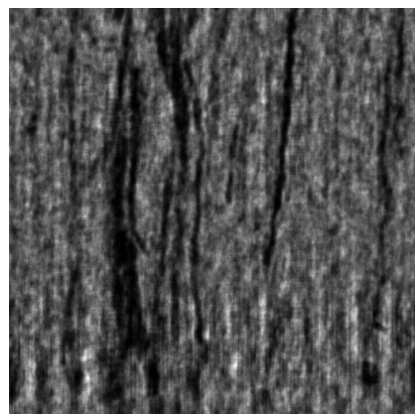

(e) WFC-HAAR

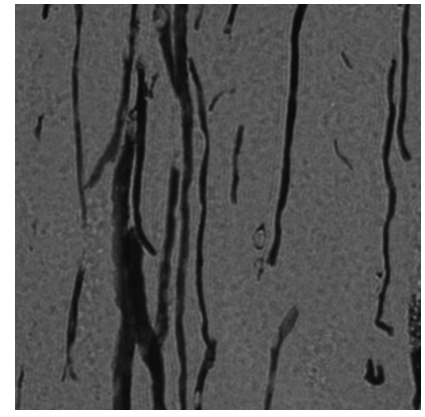

(b) Reference

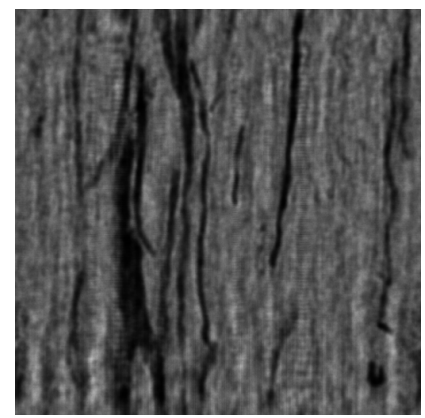

(d) WFC-AP

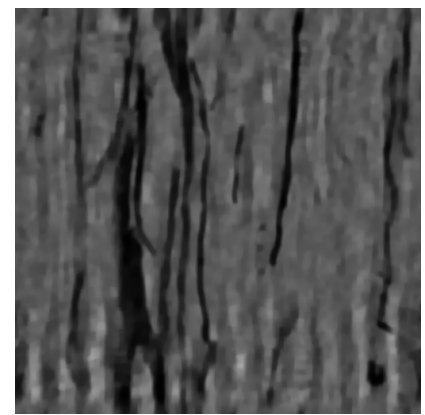

(f) WFC-FISTA

Figure 4.30. Image restoration of a image of a human kidney slice. The prominent dark streaks are blood vessels inside the kidney. The restoration makes them visible. 


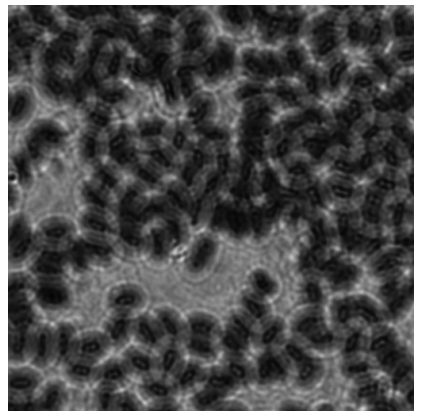

(a) Input

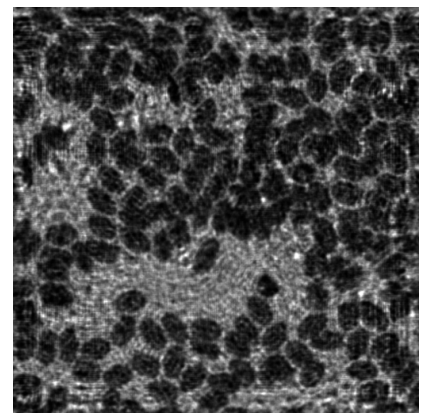

(c) WFC-GS

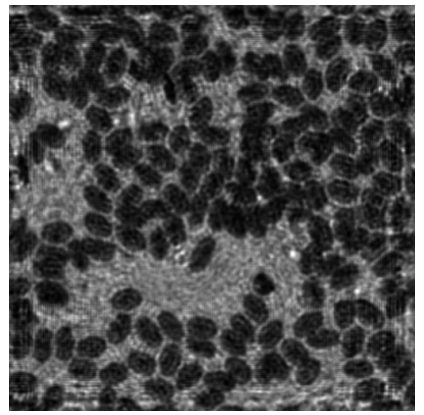

(e) WFC-AP

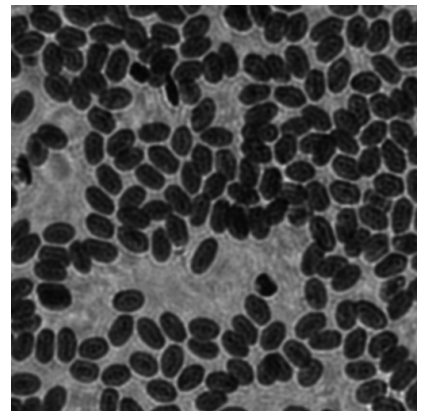

(b) Reference

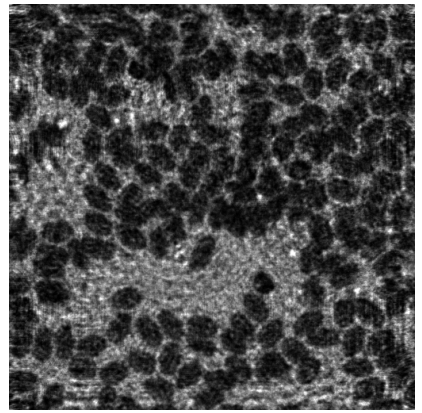

(d) WFC-HAAR

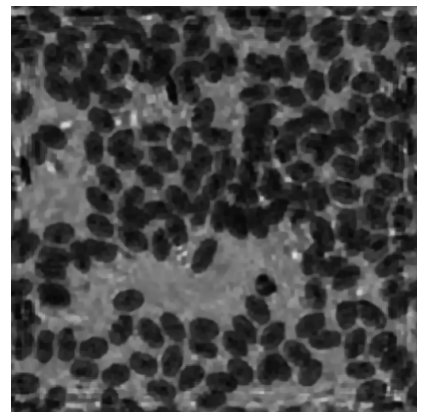

(f) WFC-FISTA

Figure 4.31. Restoration of a pigeon blood sample. The dark ellipses are individual blood cells. 
4. Applications and Results

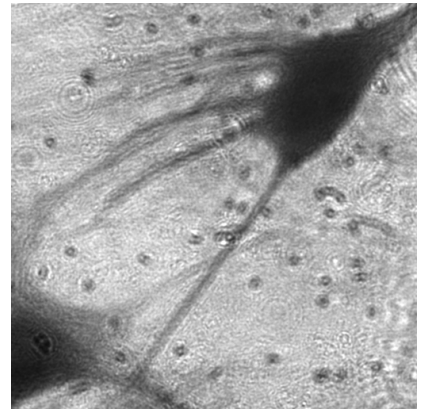

(a) Input

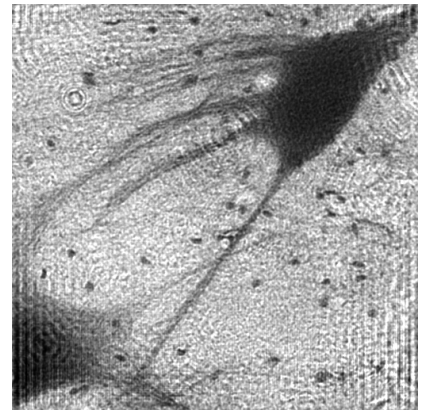

(c) WFC-GS

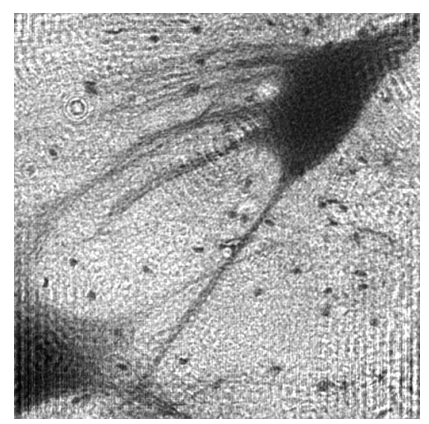

(e) WFC-HAAR

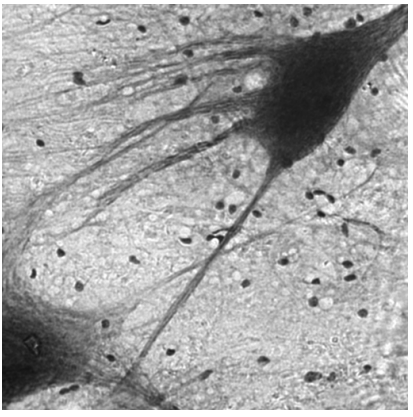

(b) Reference

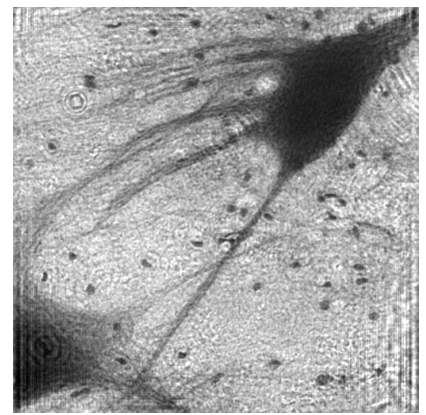

(d) WFC-AP

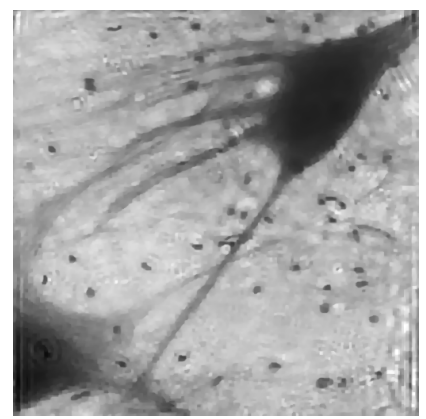

(f) WFC-FISTA

Figure 4.32. Restoration of a human neuron sample. The dark structure is a neuron. Dark dots are cell cores. Only slight defocus, imperfect restoration due to noisy background and 3D structure of the neuron. 
Plankton plays a vital part in the marine eco system. However, it is difficult to study, because the plankton size and distribution varies strongly in the water column.

The shadowgraph is an optical system for the visualization of optical density and transparency variations in transparent media.

The main idea behind the shadowgraph is that a target object is placed between camera and light source and the camera views the projected shadows of the target object. This method has advantages over traditional imaging especially in case of transparent or semi-transparent objects. The so-called "focused" shadowgraph is a well-established extension of the shadowgraph [Set01] and is used in this project, because of its improved capability to show small objects. Moreover, the optical arrangement of the focused shadowgraph with parallel illumination allows the surveillance of a large volume.

A more thorough introduction into the shadowgraph and related work about it is provided in Section 2.4.

This shadowgraph is especially built for plankton observation. An overview drawing of our current configuration is shown in Figure 4.33. A light source of small spatial extend is expanded into a beam parallel light. The shadows of this light with an observation target, in our case, plankton, are then projected into a camera and captured. A photograph of the laboratory version of the shadowgraph is shown in Figure 4.34 with highlighted illustrative light path from the illumination to the camera. Converting this laboratory built into an integrated submersible sensor is planned by GEOMAR scientists in the near future.

The illumination of the shadowgraph is a challenge, because a very bright and uniform illumination is necessary, so that the shadowgraph can be towed through the water column at a high speed (goal: up to $1 \mathrm{~m}$ per second) and at the same time a small light source is necessary to achieve the parallel light at the observation target, as well as spatial coherence. The spatial coherence is needed for the WFC- algorithms, because they are based on the coherent imaging model see Section 1.1.

Several designs of the illumination setup were tested, the first design by the company Raytrix ${ }^{8}$ is shown in Figure 4.34 in application and in

\footnotetext{
${ }^{8}$ www.raytrix.de
} 
4. Applications and Results

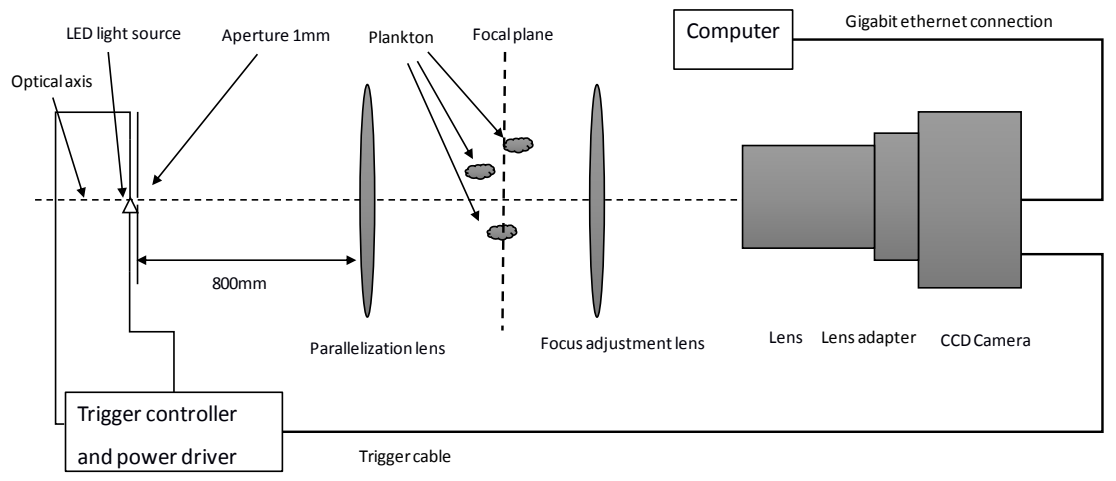

Figure 4.33. Drawing of the plankton shadowgraph system. Optical rays as in Figure 2.4 are omitted for clarity.

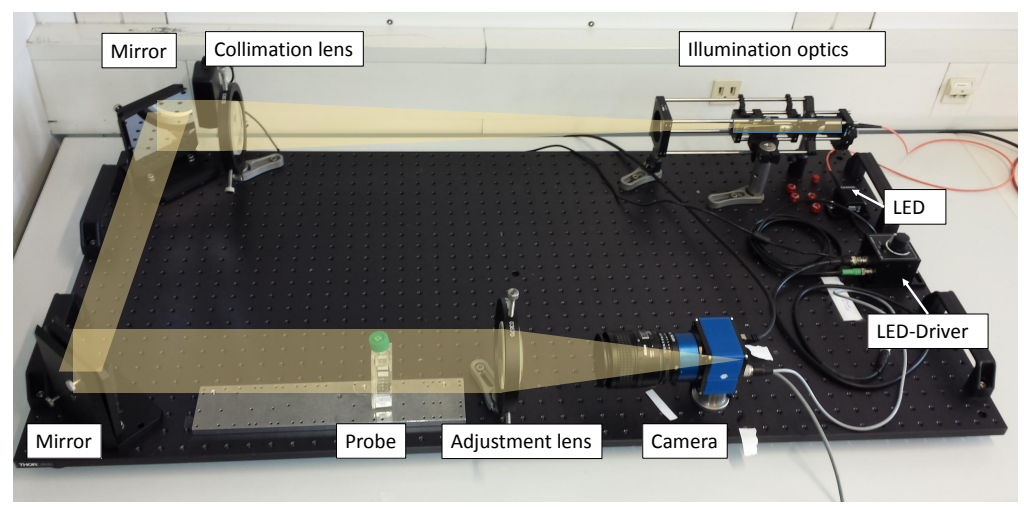

Figure 4.34. The shadowgraph laboratory setting with cuvette target. The light path is illustrated in yellow 


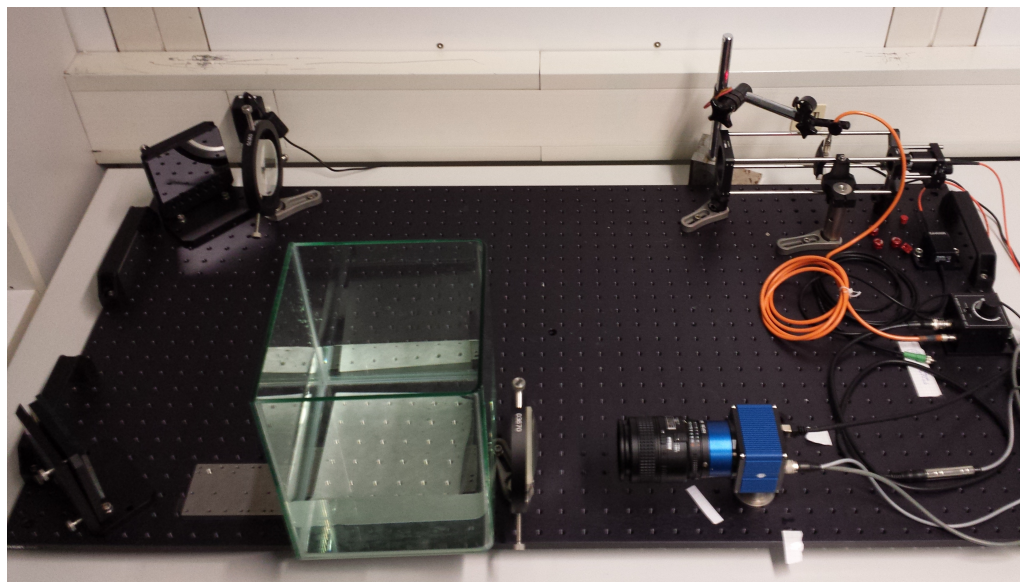

Figure 4.35. Shadowgraph with water tank .

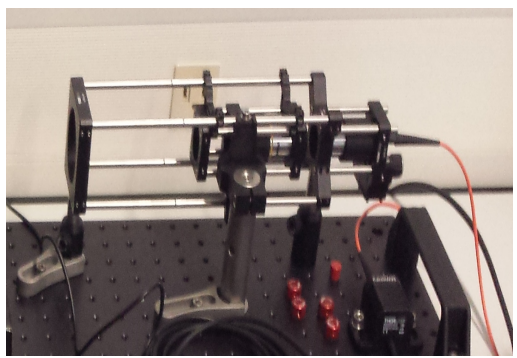

(a) Raytrix design

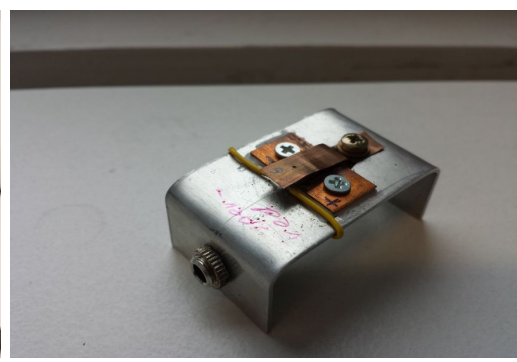

(b) Own design

Figure 4.36. Comparison of illumination sources. 
Figure 4.36a in detail. It uses a white light LED coupled into a multimode glass fiber with $1 \mathrm{~mm}$ diameter. Using two microscopy lenses the $1 \mathrm{~mm}$ exit of the fiber is projected onto a $1 \mathrm{~mm}$ aperture. With this design, the light behind the condensation lens is sufficiently parallel at the observation target.

We wanted to improve on this design to increase the light power. The goal is to reach a high light density inside a small aperture, which means smaller than $1 \mathrm{~mm}$. Coupling the LED to a fiber is limited by aperture angle of the fiber, resulting in typically less than 10\% efficiency [YK75]. Furthermore, on the exit of the fiber internal reflection occurs, further lowering the efficiency. The microscopic lenses also absorb some light, in this case it is difficult to give exact numbers, as this is highly wavelength dependent.

We have achieved a substantial efficiency improvement by avoiding all unnecessary components, using a simple design with a high efficiency LED on which a small limiting aperture is mounted. This is very similar to the setup, which we have already used on the microscope. A picture of the light source, with power supply via audio jack and two yellow cables to connect to the LED is shown in Figure 4.36b. Theoretically, the illumination uniformity of this simple illumination is worse than that of a fiber with its numerous internal reflections. However, in our application, we could not observe any changes in light intensity across the aperture in the captured shadowgraph images. Therefore, we have used the simplified light source for all our experiments.

A shadowgraph allows capturing the contours of opaque and semitransparent object in gaseous or liquid media by their shadows. A good example of a plankton observation with our laboratory shadowgraph is shown in Figure 4.37(a). The plankton can be seen very clearly with high contrast against the backlight. Marine biology research needs good resolution of coarse elements and of fine details such as antennas of copepods (the biological name of the visible plankton).

The focused shadowgraph by default has a very limited depth of field of approx. $3 \mathrm{~cm}$. This means that objects which are more than $1-2 \mathrm{~cm}$ shifted from the ideal focus plane show effects of defocus, such as ringing. This is physically defined by the design of the shadowgraph and the optical components used. The images of Figure 4.37 were taken inside the 


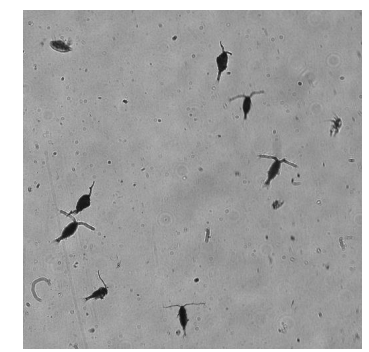

Fig. 2

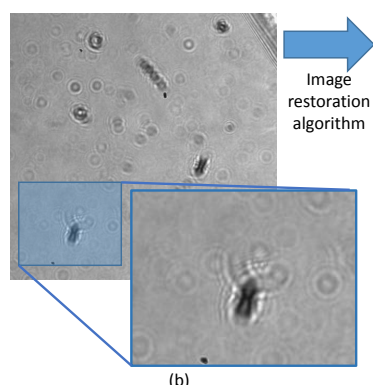

(b)

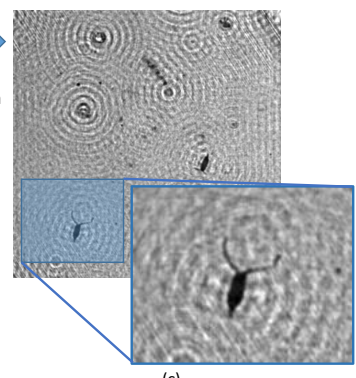

(c)

Figure 4.37. Comparison between sharp image of the shadowgraph (a), defocused image (b) and restoration with WCF-GS algorithm (c).

very limited space of a cuvette, hence all plankton is approximately at the same distance from the camera.

Figure 4.37(b) shows an example of an image where defocus effects severely downgrade the quality of the image. The antennas of the copepods cannot be seen clearly anymore.

Therefore, an image restoration algorithm is necessary to make fine details visible again and restore image fidelity. As a first test, we apply the WFC-GS algorithm. The restoration in image 4.37(c) shows very good results allowing an increased effective depth of field. Nevertheless some restoration artifacts are visible. They are the subject of the next section.

The images above where taken with the experimental setting in Figure 4.34. This setting is not yet a realistic shadowgraph setting. It uses a small cuvette as the plankton container and the measurement volume is very small. Due to the small abundance of plankton in higher depths, the shadowgraph is meant as an measurement device for larger volumes of water, therefore a more realistic experimental setting is needed and will be discussed in Section 4.4.2.

Non-laboratory, real expedition footage is provided by [Bi15]. Results with these images of a shadowgraph in oceanic application in the Chesapeake Bay are shown in Section 4.5. 


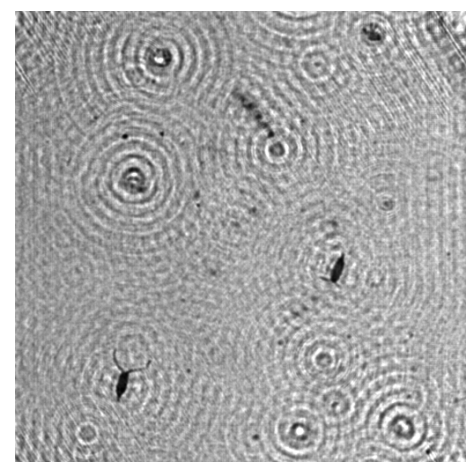

(a) Restoration with 75 iterations of WCA-GS

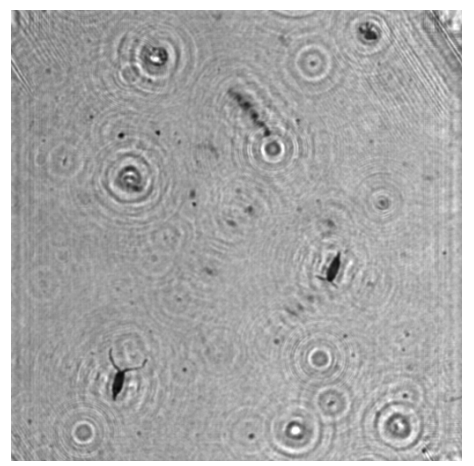

(b) Restoration with 50 iterations of WCA-AP

Figure 4.38. Comparison of WFC-GS and WFC-AP restoration.

\subsubsection{Image Filtering}

In the previous sections we have already established that restoration quality depends on the chosen WFC algorithm, and that the difference in restoration quality consists largely of ringing artifacts and noise. In fact, we saw that the ringing artifacts often diminish with an increasing number of iterations and that they may show repetitive structures. In some cases these artifacts cannot be avoided. The structure of these restoration artifacts and ways to remove them by empirical post-processing are the focus of this section.

We start using images acquired with the shadowgraph as illustrative examples in this section. Already established in previous sections and clearly visible in these shadowgraph images is the difference between the WFC-GS and the WFC-AP algorithm as seen in Figure 4.38. To make the structure of the ringing artifacts more visible we perform the DCT (discrete cosine transform) on the restored image and visualize the spectrum. The DCT as a variation of the Fourier transform is chosen because of the clearer visualization of the spectrum, because of the better axis orientation.

Figure 4.39 shows the logarithmic absolute value of the DCT from the restoration in Figure 4.38a. Row wise the amplitudes of horizontal spatial 


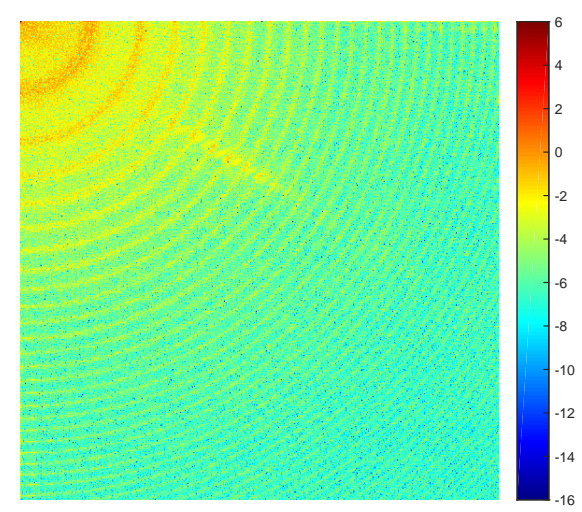

Figure 4.39. Logarithmic absolute of the DCT-spectrum from the restoration in Figure 4.38a. Horizontal spatial frequencies in $\mathrm{x}$-coordinates, vertical frequencies in $y$-coordinates. The origin is in the top left corner.

frequencies from low to high are shown and column wise the amplitudes of the vertical spatial frequencies, the constant part is in the top left corner. Clearly visible is a strong the repetitive ring structure that is formed by the restoration artifacts.

In the next step we want to design a filter that suppresses these frequencies. The first observation about these rings is that they are circular and therefore the spatial spectrum has circular symmetry. This can also be derived directly from the circular interference rings in Figure 4.38a. The rings in Figure 4.39 are not equidistant, but the distance is increasing with the frequency. Hence, we need a filter that matches this characteristic.

This description matches that of the linear chirp function. The timeseries of a chirp function with lineary increasing frequency, where the frequency $f_{i}$ at timestep $t$ is defined by the starting frequency $f_{0}$ and end frequency $f_{1}$, is given by:

$$
f_{i}(t)=f_{0}+\frac{f_{1}-f_{0}}{f_{1}} t .
$$

We define the chirp $c h i_{r}$ as the timeseries of 512 steps beginning with 
4. Applications and Results

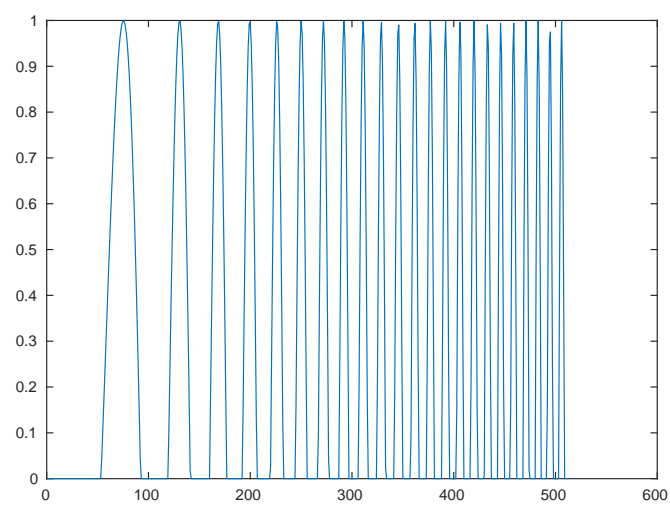

Figure 4.40. Plot of the chirp function used as a frequency filter with absolute $c_{\text {filter }}$ values on the y-axis across the 512 datapoints.

frequencies of 0 and ending with empirically defined frequency 0.09

The filter itself ' $c_{\text {filter }}$ ' is set to

$$
c_{\text {filter }}=-1 \cdot \max \left(c h i_{r}\right) .
$$

A plot of this 1D-filter is shown in Figure 4.40.

The filter shall be applied directly in the Fourier domain by eliminating the linear increasing frequency components of the ringing artifacts. We need to consider that the DCT spectrum is two-dimensional. Because the horizontal and vertical frequencies of the artifacts match, we apply the 1D filter as a field $c f$ for horizontal and vertical coordinates $x, y$ of the spectrum:

$$
c f(x, y)=c_{f i l t e r}\left(\sqrt{x^{2}+y^{2}}\right) .
$$

Now the filter (Figure 4.41a) can be multiplied point wise on the DCT of the image.

A visualization of the filter and of the filtered result formed by multiplication of filter and input image in the Fourier domain, is shown in Figure 4.41. Afterwards the inverse DCT is applied to form a filtered result image, see Figure 4.42 . 


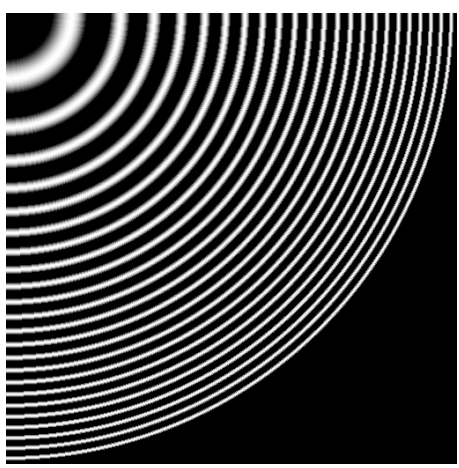

(a) 2D chirp function, white colored pixels show values close to one, black colored ones show values close to zero.

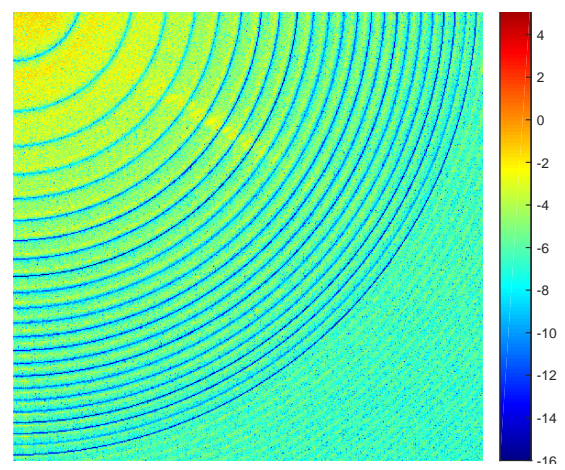

(b) Filtered result

Figure 4.41. DCT of 2D-chirp function and multiplication of chirp and restoration. Horizontal spatial frequencies in $\mathrm{x}$-coordinates, vertical frequencies in $\mathrm{y}$ coordinates. The origin is in the top left corner.

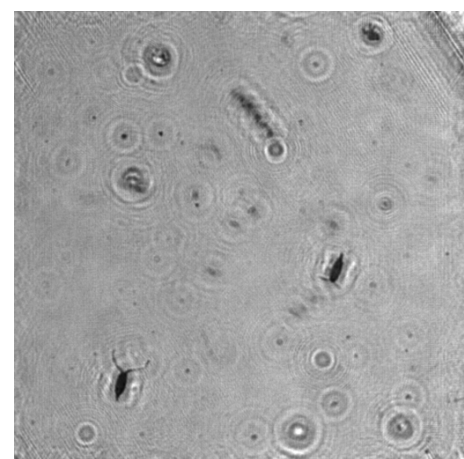

(a) Filtered version of WFC-GS re- (b) Filtered version of WFC-AP result sult(Image 4.38a)

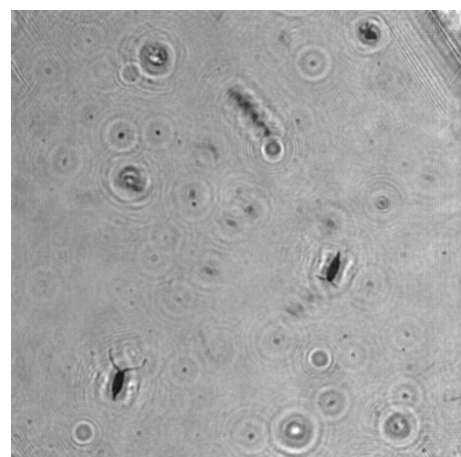

(Image 4.38b)

Figure 4.42. Filtering result of WFC-GS and WFC-AP. 
Large improvements in image fidelity with filtering can be seen especially for the stronger restoration artifacts of WCA-GS. For the less strong artifacts from the WFC-AP the effect is not as strong. In fact, the images in Figure 4.42 look very similar. This means with our chirp filter post-processing even an inferior algorithm can be chosen and we can still achieve very good results.

For other images with a similar setting, the same filters can be applied, fine-tuning is necessary only if the imaging conditions are changed drastically.

\subsubsection{WFC-Autofocus for Spatially Variant Blur}

In the previous section the plankton was placed inside a small cuvette and therefore only a small depth of field was required. Now towards a more realistic setting the cuvette is replaced with an aquarium, in which the plankton can swim freely. A picture of the aquarium is shown in Figure 4.35. By acquiring an image with the camera of the shadowgraph, it can be seen that some plankton is in focus, but most plankton is out of focus and not with the same amount of defocus. The blur is spatially variant.

For more convenient testing and simulation of this effect, an artifical compound image is composed. A cropped image of a single sharp plankton is stitched together with 15 copies with increasing defocus by row and column. For illustration this image is depicted in Figure 4.43. Now the goal is restore each sub-image of this compound image, even though the defocus varies.

The WFC algorithm is very fast, hence instead of an optimization loop and to avoid any problems with local minima of any sharpness criterion, the restoration is attempted with different radii in small steps over a sensible range. This results in a virtual focal stack, quite similar to a focal stack created with traditional photography. Now a sharpness criterion is applied on all images in the stack and a robust maximum is determined.

To this effect, we begin with creating a focal stack of restorations, starting with no deformation, increasing spherical wavefront deformation is compensated with the WFC-algorithm. A more graphic description of this process is that the virtual focus plane is shifted with every image 


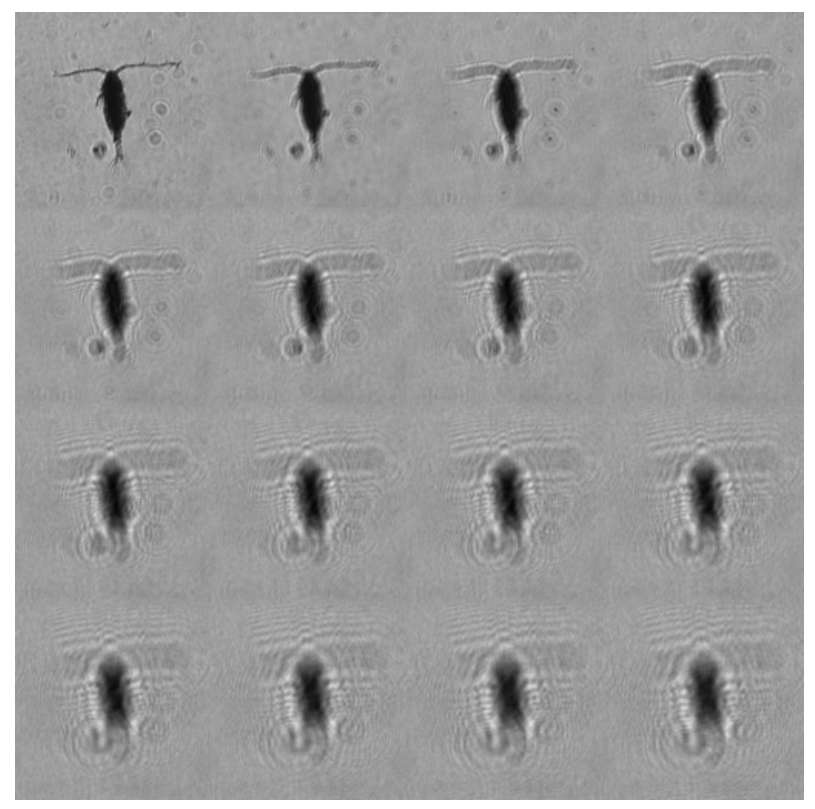

Figure 4.43. Artificial test image with spatially variant defocus. The top left partial image is sharp, the defocus is increasing by row and column.

by a small amount. As shown in the beginning of Chapter 4, defocus is a symmetric effect and images in the focal stack before and behind the focus plane are identical. Thereby, we only need to scan in one direction, meaning that the sign of the additional wavefront is irrelevant. For a given image the restorations in small steps of defocus are saved in memory for further processing.

These images form the restoration stack for the illustrative test image, that is depicted in Figure 4.43) are shown in Figure 4.44. The original test image is shown in the top left corner. In this image the top left plankton is sharp and all others are increasingly defocused. The following images are from the focal stack with increasing defocus compensation.

In the second image this results in the focus plane being shifted to the top right plankton, which is now depicted sharply. In image three the third plankton in the second row, in 4.44d it is the second in the third row, 
in $4.44 \mathrm{e}$ the first in the fourth row and in the last, $4.44 \mathrm{f}$ the fourth in the fourth row. Not all images from the focal stack are shown, but it becomes clear that for any defocused plankton, there is a focus plane, onto which we can refocus.

Now the goal is to detect the sharp image of the plankton for any given configuration of focus. To do this the following procedure is presented.

From the first image, which is the given input image acquired with a camera, or simulated for illustration purposes in this case, the plankton individuals are segmented using Otsus threshold [Ots79] and the opening morphological operator. The bounding box around each object is calculated and very small objects are dismissed, so that every bounding box contains a plankton individual. For every bounding box, the goal is to find in which layer of the restoration stack lies the sharpest image. Thus, the sharpness criterion is applied on the bounding box on every layer and the entry with the highest score selected.

Different algorithms for sharpness detection were introduced in Section 3.7. As a first test, we apply the normed variation criterion of [SDN04]. This criterion works well on incoherent images and selects all the sharpest instances of the restored image from the simulated blur. In the next step, we will apply the criterion on a real shadowgraph image with defocus (Figure 4.45) and see that the criterion is inappropriate to these images.

For the shadowgraph due to very challenging imaging conditions, the criterion must be robust to noisy input and imperfect restorations. The properties of sharp plankton images to be restored are clear. The plankton is uniform dark object with a clear boundary and high gradient to the bright background. As explained in the general introduction of autofocus in Section 3.7, this prior knowledge allows the definition of the following criterion

$$
\text { value }=\sum_{i=1}^{n} \sum_{j=1}^{m}|\nabla I(i, j)|^{2} \exp (I(i, j))
$$

where $I(i, j)$ denotes a pixel value in image $I$ with size $n \times m$. Our reasoning behind this score is as follows. The gradient is a measure of contrast, for better discrimination it is applied to the squared image. Because the direction of structure does not matter, we only consider the absolute value of this gradient and weight brighter regions higher. This is scaled with the exponential function of the image. Afterwards the sum over the individual 


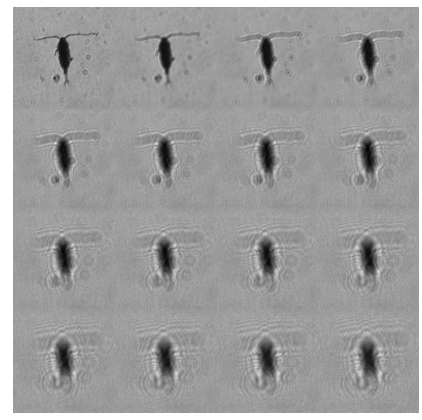

(a) Original image

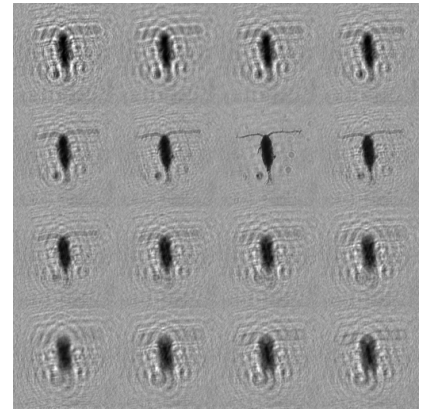

(c)

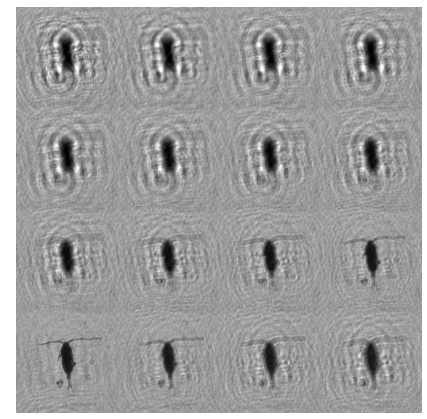

(e)

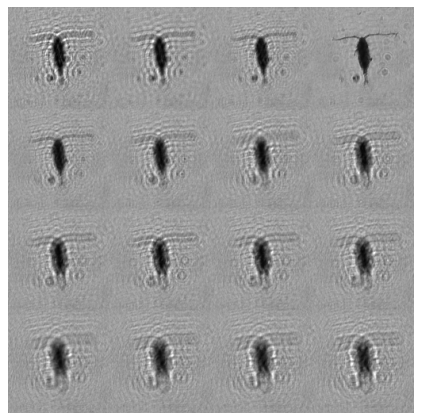

(b)

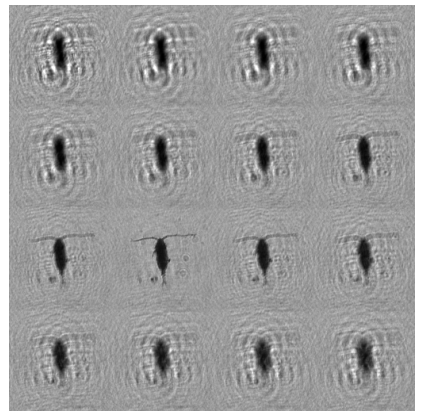

(d)

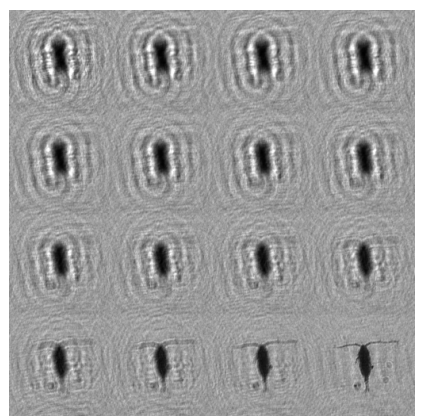

(f)

Figure 4.44. Restoration stack of simulated image with spatially variant blur only 6 are shown as demonstration. 
4. Applications and Results

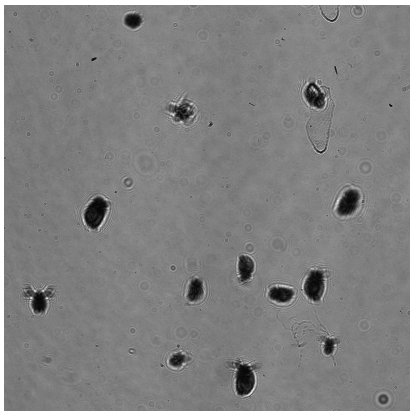

(a)

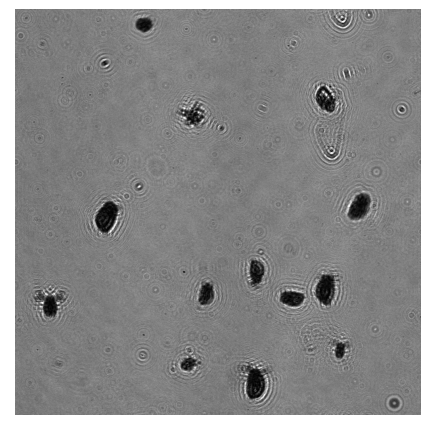

(c)

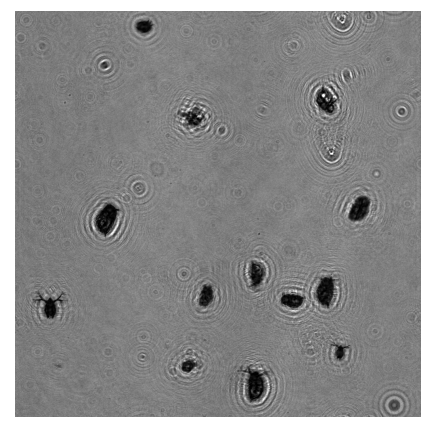

(e)

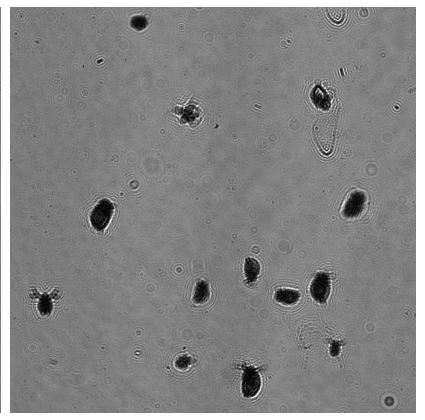

(b)

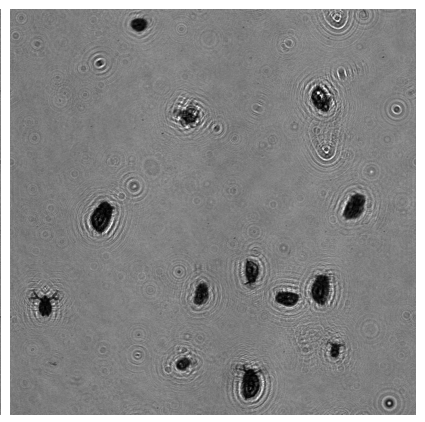

(d)

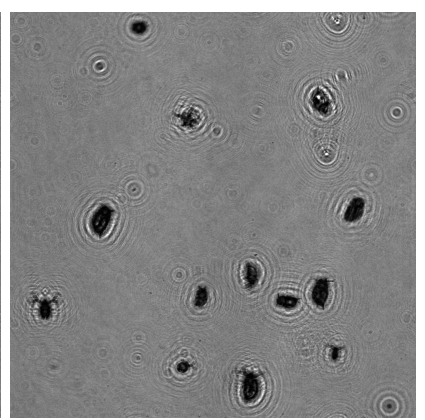

(f)

Figure 4.45. Restoration stack with increasing defocus correction on shadowgraph image with spatially variant blur, only 6 images of 60 are shown. 

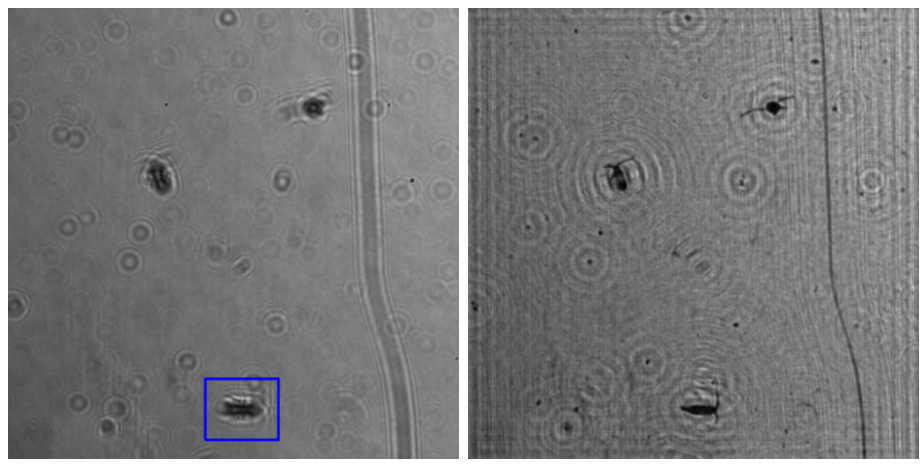

(a) Original image with crop (b) Restored image with correcused for sharpness measurement tion value of 25, WFC-AP with indicated in blue 100 iterations

Figure 4.46. Shadowgraph image for sharpness criterion evaluation.

values for each pixel is calculated to reach a measure for the entire image. Note that the image is made up of gray scale floating point values between 0 and 1 . The exact definition of the criterion was empirically explored.

To test both criterions we use a restoration stack with 40 different configurations from the shadowgraph image in Figure 4.46 and use the blue highlighted crop in the resulting restorations as the input for the criterions. Visually we determined that the image is sharpest at a correction value of 24.8. The image was chosen because the sharpness can be easily seen at the black scratch on the cuvette on the right hand side of the image. We used the WFC-AP algorithm with 100 iterations and slight ringing can be seen in the restoration. This is a realistic situation for testing the algorithms.

The value of the criterions is calculated for every restoration in the virtual focal stack. A graph of perceived image sharpness with the novel criterion in Equation 4.4.4 and variance criterion [SDN04] (Equation 3.7.1) is shown in Figure 4.47. The strength of the spherical wavefront correction on the $\mathrm{x}$-axis is noted as maximum path difference of $\lambda$.

The graph of the criterion values with criterion [SDN04] does not show a minimum at the correct value. It is not able to find the correct correction 
4. Applications and Results

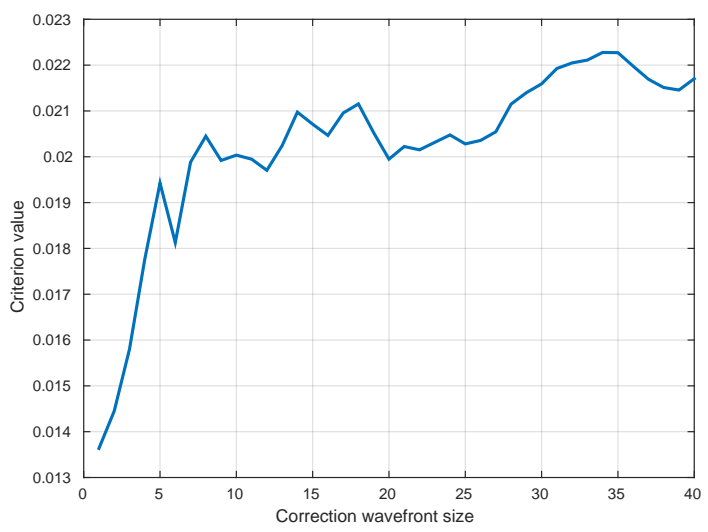

(a) Normed variance [SDN04] criterion

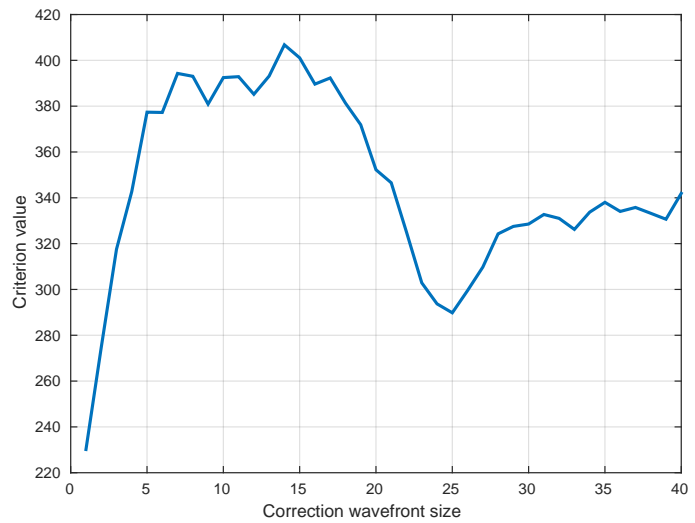

(b) Specialized shadowgraph criterion, defined in Equation 4.4.4

Figure 4.47. Image sharpness as measured per criterion for restorations of the selected image patch (Figure 4.46) with different wavefront aberration estimations. 
wavefront setting for plankton shadowgraph images and is therefore not suitable.

In contrast, our shadowgraph sharpness criterion shows better results. The local minimum at value 25 matches with the empirical observation that the sharpest image is achieved with this value, see 4.47. Nevertheless, it fails to globally find the sharpest instances from a restoration stack, because the criterion values for low correction wavefront values are even lower. This means that the criterion is not robust. The criterion could be improved by adding several heuristic image specific rules, however instead of performing parameter tuning for every setting, we want a criterion, which detects sharp shadowgraph images without further input. Therefore, further attention is placed on this topic in the next section.

\subsubsection{Image Sharpness Classification with Deep Learning}

An automatic determination of defocus requires the information about when an image is sharp and when it is not. Evaluating a gradient based term or variance is good enough for incoherent images, however such an approach fails for real coherent images, where it is unclear if large gradients are caused by noise or if sharp contours are caused by ringing and not by the object. Clearly specifying how such a sharp image is defined, if noise, ringing and non-ideal imaging conditions are considered is difficult. Nevertheless, the task itself does not seem difficult for a human, because for an human observer it can be easily seen, if an image is sharp.

Hence, our solution to this problem is to employ deep learning methods. Deep learning is a machine learning technique that uses deep networks of artificial neurons to imitate the possibilities of the human vision. In fact, the structure of artificial neural networks is inspired by the human cortex [IK01].

The given problem can be seen as a regression problem of determining a sharpness score for an image. With the exception of special test images, it is hard even for a human observer to give an objective sharpness score to a natural image. Because the feasibility of solving a problem using deep learning depends heavily on the availability of training data, we develop a different approach and consider the task a classification problem.

Our approach is to create a deep learning classifier that is able to 


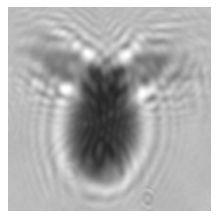

(a)

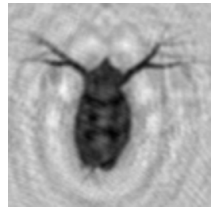

(f)

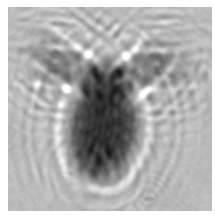

(b)

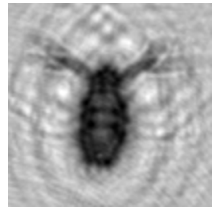

(g)

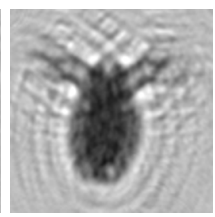

(c)

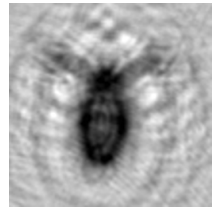

(h)

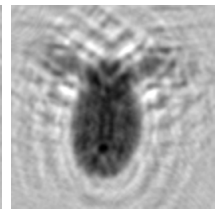

(d)

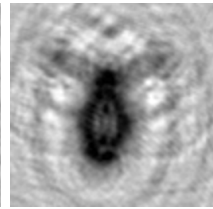

(i)

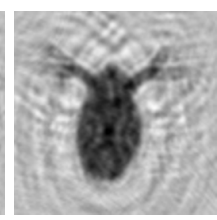

(e)

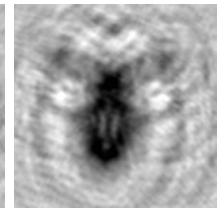

(j)

Figure 4.48. Training images generation with a region of interest throughout a focal stack of restoration with increasing defocus aberration correction. Image 4.48a is from the original image, while image $4.48 \mathrm{f}$ is the sharpest image and is sorted to the sharp image training examples. All other images are unsharp and are used as training examples for the unsharp class.

discriminate sharp images from blurred image. It should work in such a way that for any image a classifier can give a probability for whether the given image belongs to the class of sharp images or to the class of unsharp images. The reason behind this approach is that a classifier is much easier to train than a regressor, as it can learn from examples of sharp images and unsharp images, which must be of sufficient count and diversity.

Creating this training set of images is the main challenge. In order to create the training examples, restorations with 60 increasing steps of simulated defocus are saved in a focal stack, similar to auto-focus on simulated images. Then for all plankton particles in an image, a bounding box is found. This bounding box defines a region of interest, which is isolated, scaled to the same size and saved for every restoration in the focal stack. This means we now have 60 images for each object and from one to three must be categorized as sharp, while the rest is unsharp. An example is shown in Figure 4.48. This selection of "sharp" in the end defines the discriminative power of the classifier, or with other words, how sharp it separates the two classes. 

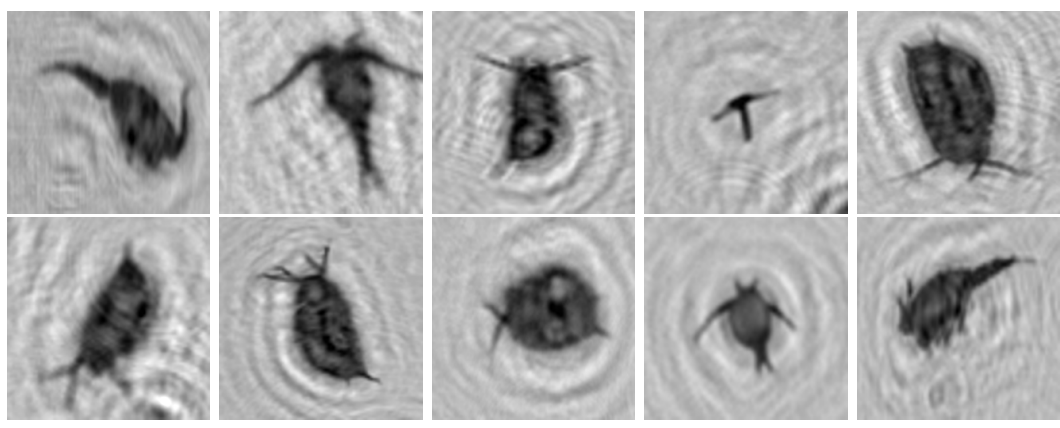

(a) Sharp images
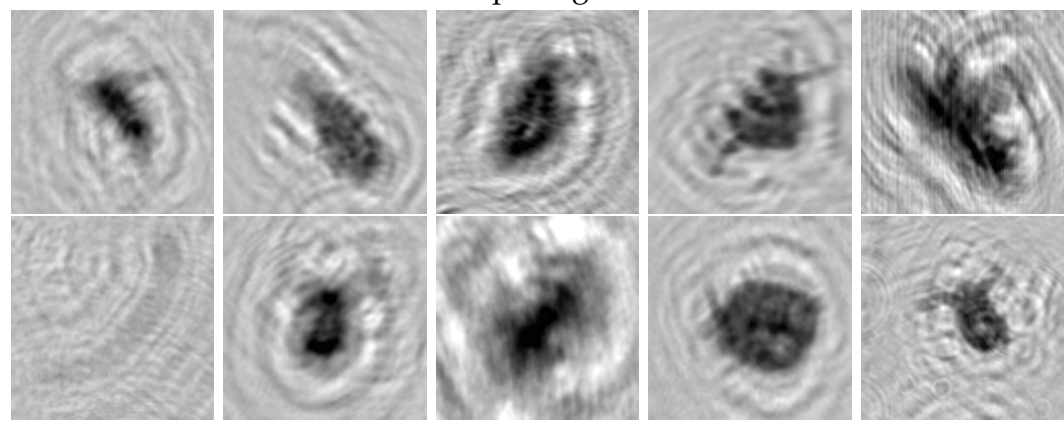

(b) Unsharp images

Figure 4.49. Sharp and unsharp examples from the training set

With this technique 463 sharp images and a much larger number of unsharp images were obtained. To increase training data further data augmentation was used and all sharp images were rotated or flipped, effectively multiplying the image count by four. While the data augmentation is already a step in the right direction, the training data is still heavily unbalanced. Thus, for further use all sharp images and only randomly selected unsharp images of equal count are used, such as in Figure 4.49. From these, all extracted examples originating from two training images are isolated as validation data, to be able to observe the learning process later on.

For the neural network itself, we require a network architecture, which 
allows a good sharp/unsharp classification. Furthermore, we want to evaluate, if increasing network complexity has benefits for this task. We want to see, if the network is the limiting factor and whether designing and tuning a network tailored to our task is a meaningful or useless undertaking.

We start with the network architecture Caffenet, a variation of the very popular Alexnet [KSH12] is chosen with randomized crops of $100 \times 100$ pixel size from the training images as input. The Alexnet won the 2012 Imagenet challenge [RDS+15], in which the task was to assign 1000 object category labels to 150000 natural images. Thus, we believe that this architecture should be powerful enough handle our problem. The differences between Alexnet and Caffenet are negligible to the performance ${ }^{9}$. Caffenet has a very straight forward design of convolutional and pooling layers, as seen in Figure 4.54.

During training the learning rate is adjusted so that in the beginning large progress is made and nearing the optimum the changes are decreasing. To this effect the learning rate changes with a stepwise learning rate decay strategy, which means that the learning rate is lowered every few epochs as shown in Graph 4.50.

For training we use stochastic gradient descent with a batch size of 32. The validation loss is the value of the loss function for validation data and the training loss is the value of the loss function on training data. The training loss is also the value, which is optimized by using training data only, while the neural networks power of generalization is tested by comparing it with the validation loss. The network training graph in Figure 4.51 shows a very good training results with a validation accuracy of over $95 \%$. Because the sharp or unsharp differentiation for training and validation data is done subjectively by the author and includes corner cases, this number signifies the agreement between the neural network and the human author on image sharpness. This value is very high. Some over-fitting can be seen in the final iterations, visible by the fact that the validation loss is stable, while the training loss reaches almost zero. This is overcome by applying the early stopping strategy and using the model in iteration 50 as the final result. For all timing measurements we employ

\footnotetext{
${ }^{9}$ For an explanation of the differences see https://github.com/BVLC/caffe/issues/4202.
} 


\subsection{Focused Shadowgraph}

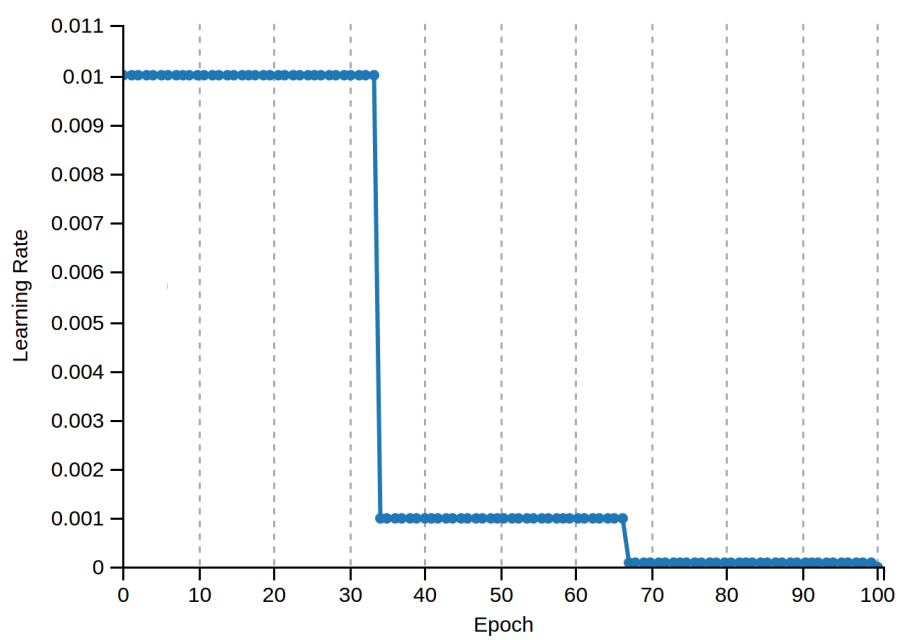

Figure 4.50. Learning rate decay in three steps from 0.01 to 0.0001 .

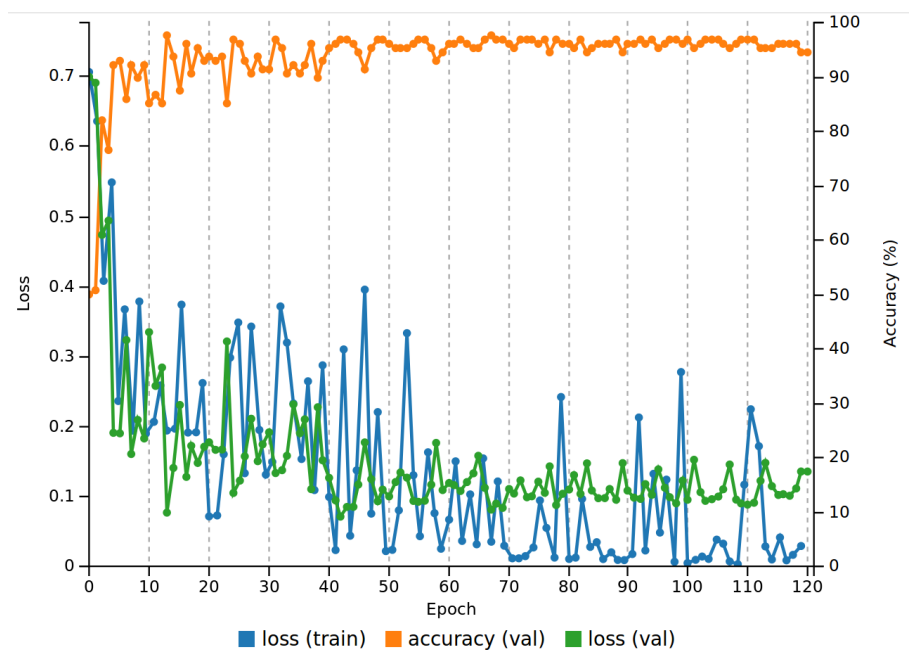

Figure 4.51. Network training graph on Caffenet 


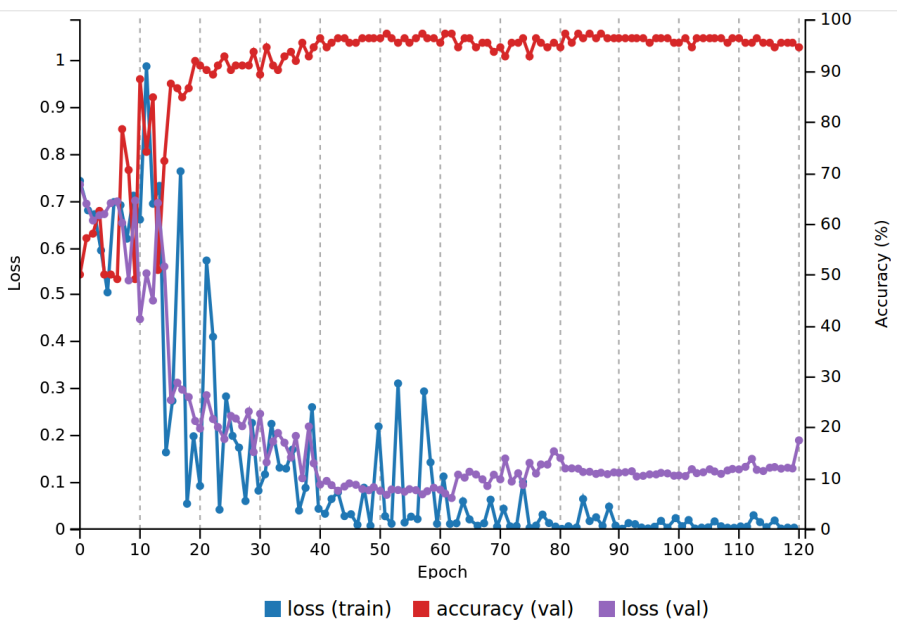

Figure 4.52. Network training graph with Googlenet.

a Nvidia GTX970 with the caffe neural network library [JSD+14]. The training time for Caffenet is 9 min 12 second.

As the next architecture we evaluate the topscoring Imagenet challenge [RDS+15] architecture of 2014 the 'Googlenet' [SLJ+15]. It consists of a 22 layers, however the general architecture is not as flexible. The number of free parameters is decreased by the so-called inception modules, which is the concatenation of convolution kernels with different size, for details see [SLJ+15]. Figure 4.54 shows the differences between Caffenet and Googlenet in neural network complexity.

The training graph for the Googlenet architectur is shown in Figure 4.52. Training time for the epochs shown in the Graph with Googlenet is $48 \mathrm{~min}$ $34 \mathrm{sec}$ on $256 \times 256$ input images. This is significantly slower than Alexnet, but still acceptable, because the network only needs to be trained once. The results with the Googlenet architecture are very similar to those with Caffenet, with almost the same classification accuracy. The Training loss reaches zero in later epochs, while the validation loss starts to increase after iteration 50. Although the validation accuracy remains high, this indicates strong over fitting in the later epochs, which means that the loss 


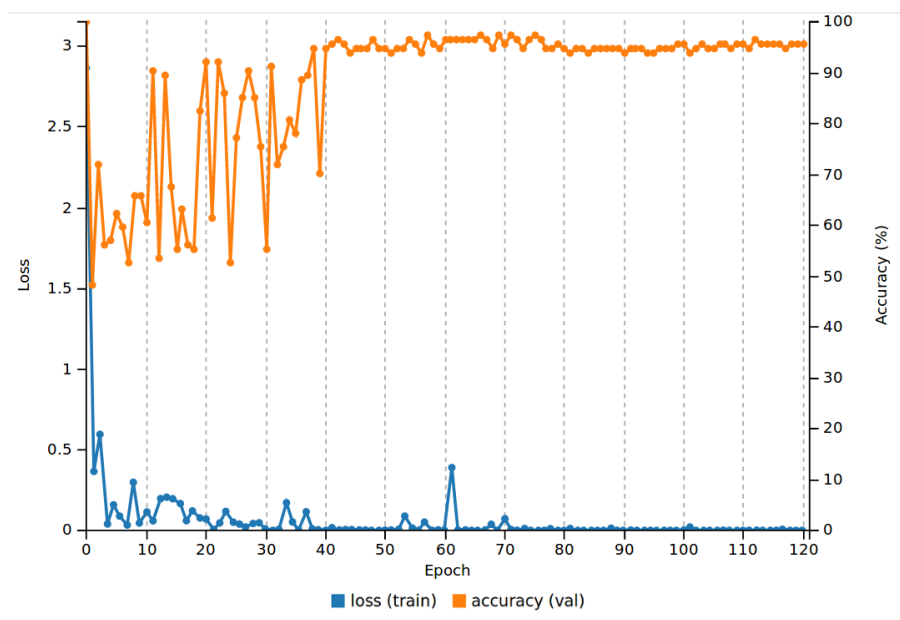

Figure 4.53. Network training graph with Resnet 32.

values might not be correct for validation data. We apply the same early stopping strategy and propose using a model from iteration 40.

As a validation we also tested the 2015 Imagenet classification top scoring architecture the 'ResNet' [HZR+16] in a 32 layers implementation. The training graph in Figure 4.53 confirms that we are not limited by the network architecture.

Because the accuracy of all tested architectures is the same and Caffenet is slightly faster in our implementation, this is the network architecture we choose for the following evaluations.

\subsubsection{All-in-Focus Images and 3D View}

In this section the process of generating an All-in Focus image is described. An All-in-focus image is an image in which all parts of an image are depicted sharply. The image is generated computationally, so even if it is physically impossible to acquire such an image with a camera image, because different parts of the scene are located at different depth, all parts are appear sharply.

In the first step a blank image is prepared, which serves as the frame 


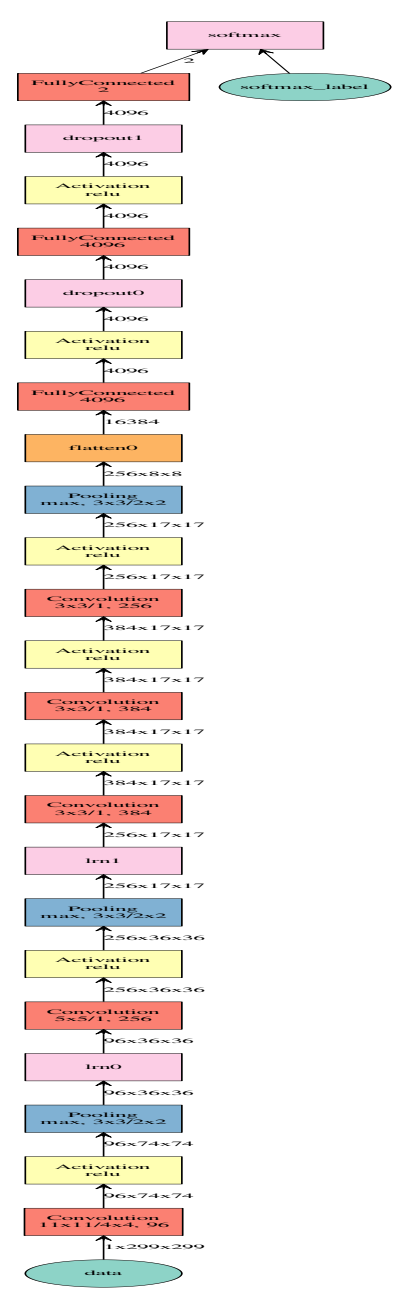

(a) Caffenet 2012 [KSH12]

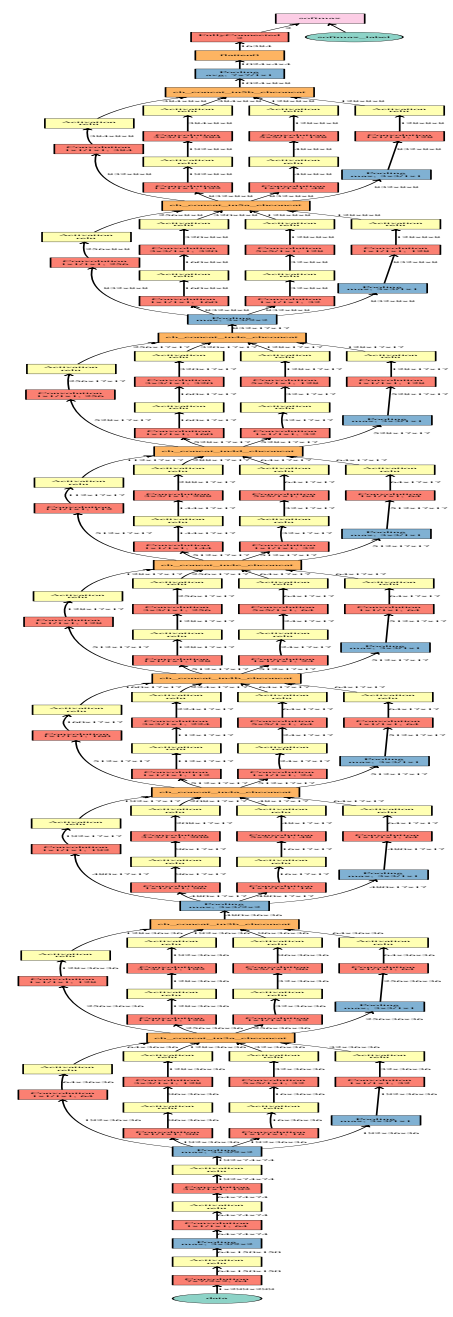

(b) Googlenet 2014 [SLJ+15]

Figure 4.54. Comparison of CNN architectures, visualized with automated layouting, scaled to fit. In cyan: Input data layer, in red: Convolution or fully connected layers, in yellow: Activation layers, in blue: pooling layers, in orange: Flattening/concatenation layers, in pink: Normalization, dropout and loss layers. 


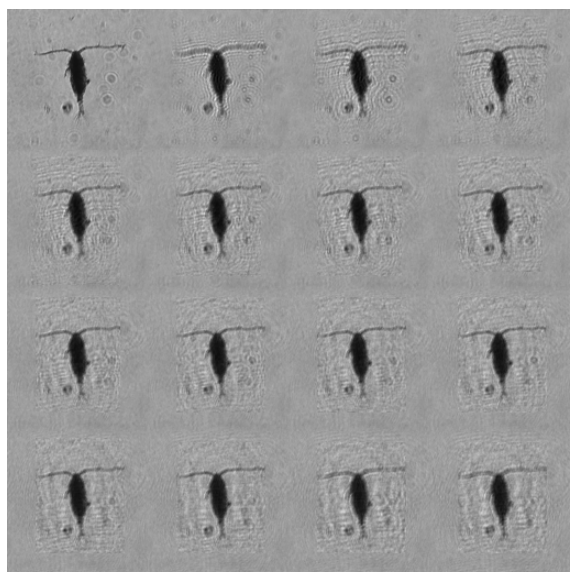

Figure 4.55. Simulated all-in-focus image.

for sharp image parts that will be collected in the next steps.

As first, a virtual focal stack for different depths for the entire image is generated, by applying the WFC-algorithm with increasing spherical defocus compensation configurations. Then the bounding boxes around plankton are found with Otsu's method as described in the previous section. For all bounding boxes in the focal restoration stack the image contents of the sharp layer according to the neural network are then selected and copied into the prepared frame. If more than one layer is classified sharp, the one with the highest as sharp classification rate is chosen. This process is repeated for all bounding boxes.

For all pixels in the prepared frame which remain blank, we know that they should be background pixels, thus pixels from the original image are inserted. This all-in-focus image from the collage of sharpest bounding boxes is the first result of this method. Applied to the simulated focal stack in Figure 4.44, the result is the all-in-focus image Figure 4.55. We can clearly see that for all plankton in the simulated image the sharp restoration is selected. The simulated image was created by multiplying a small tile and shows the same object 16 times, this also becomes visible in the restoration.

For a shadowgraph image, the all-in-focus image with the best in-focus 
images selected using the trained deep learning sharpness classifier is shown in Figure 4.56. For the focal stack the WFC-FISTA algorithm was used. Figure 4.56 also shows the raw input image. The difference between both images shows best the power and usefulness of all-in-focus images. The plankton can now be measured, because they are now clearly delimited by sharp edges and can be recognized as individuals by their shape and antennas. Due to the imperfect imaging conditions, some ringing artifacts are visible around the restorations. The effective depths of field of the entire shadowgraph system is extended from $2-3 \mathrm{~cm}$ to $20 \mathrm{~cm}$, in this case limited by the size of the aquarium.

The defocus radius of the sharpest layer also has a physical meaning. This parameter value obtained by the auto-focus criterion determines the distance from the focus plane and thus encodes the depth of the plankton, allowing a three-dimensional plankton rendering. Because defocus effects before and behind the focus plane are symmetric, there is an ambiguity. By setting the reference focus plane a few millimeters before the tank, this ambiguity is solved and we can assume that all objects are located behind the reference focus plane. The image in Figure 4.56 is such an image (this acquisition strategy is the reason why no plankton objects appear sharp in the raw image). This allows assigning the defocus value of the sharpest instance in the restoration stack to the bounding boxes. These defocus values are then converted to metric units of depth by observing the necessary wavefront deformation on a test target at the front of (small values) and behind the tank (large values). Finally, a 3D rendering of the bounding boxes with its sharpest image at the correct distance can be made as shown in Figure 4.57. This 3D view of the plankton volume is generated from a single shadowgraph image and made possible by the WFC-algorithm.

In Figure 4.58 the all-in-focus images and 3D visualization of another shadowgraph image is shown. We see that almost all plankton is restored sharply. A failure case can be seen in the left most bounding box in the 3D visualization, the neural network was unable to correctly focus the object, however it is hard to decide whether this is plankton at all. For other dirt and plankton particles the restoration works flawlessly, for example the particle of the third bounding box in the 3D view from the right, can only be clearly identified as dirt in the restoration image. 
4.4. Focused Shadowgraph

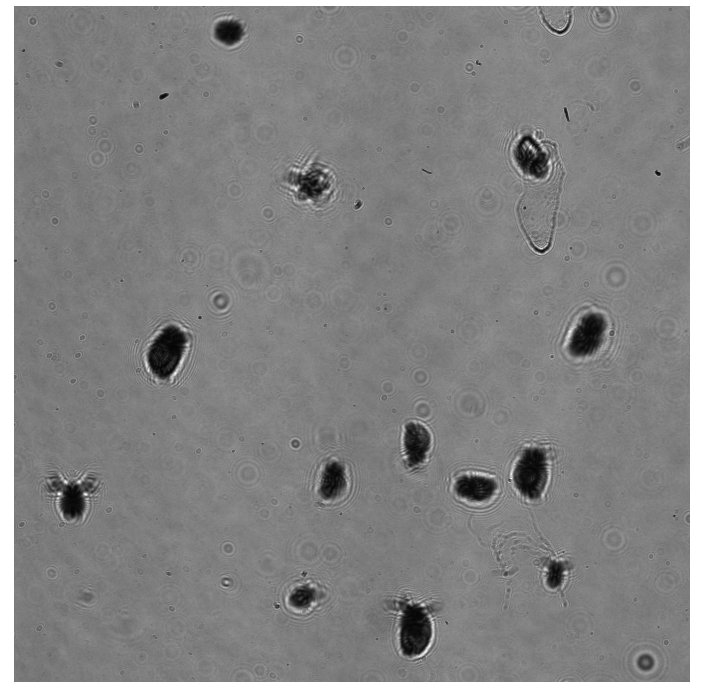

(a) Input image

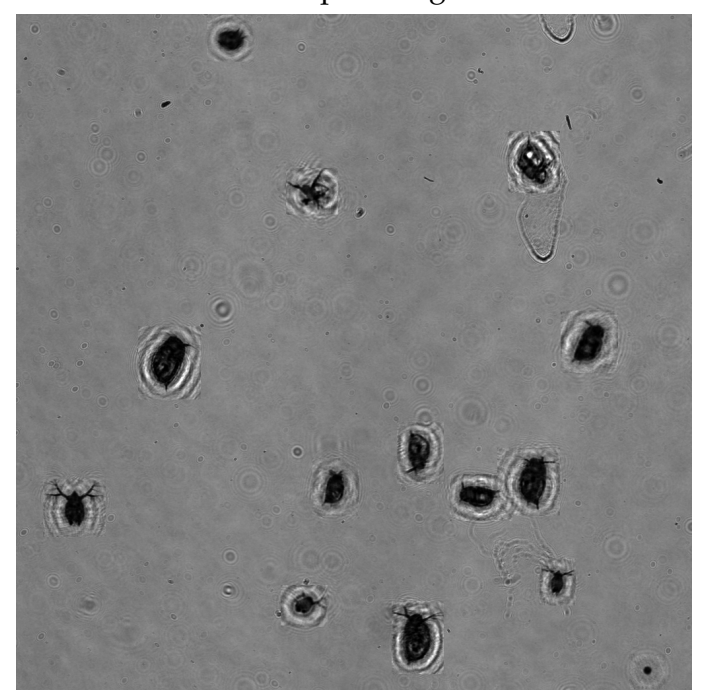

(b) All-in-focus image

Figure 4.56. Comparison of shadowgraph and all-in-focus image. 


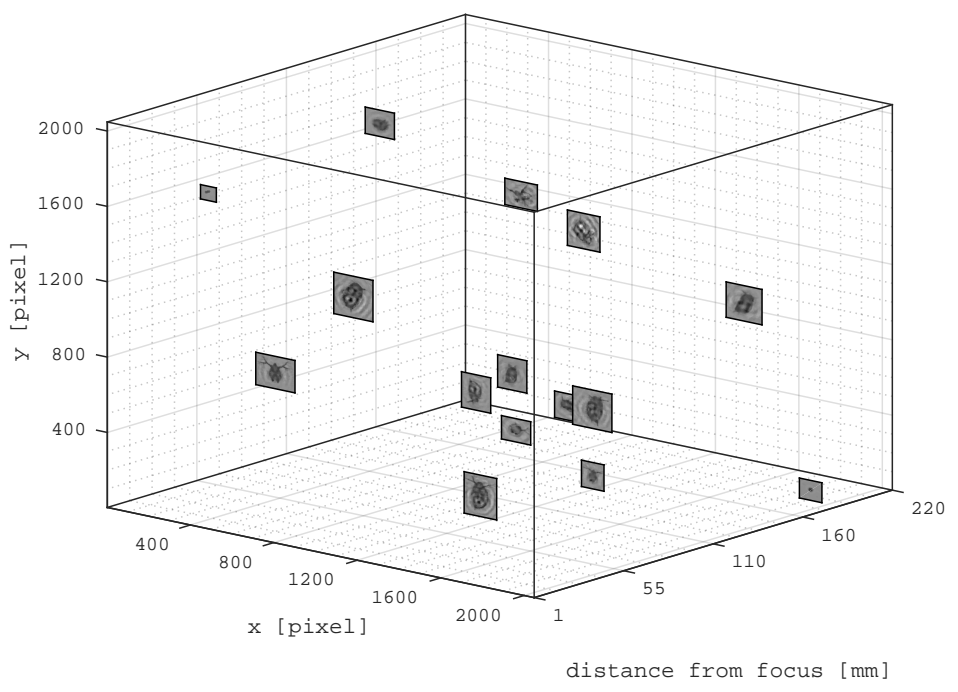

Figure 4.57. 3D visualization of depth from defocus.

\subsection{Shadowgraph in Marine Application}

In the previous section we developed our own shadowgraph system and designed algorithms for its evaluation. We tested our algorithms on simulated images and images acquired with that laboratory shadowgraph. In this section we want to extend this work on images acquired in real oceanic conditions. These images were acquired in Chesapeake Bay in the Atlantic of the coast of Virginia by Bi et al. in October 2011. The system used is the ZOOplankton VISualization (ZOOVIS) shadowgraph system [TMB05] for plankton observation. A picture of the system is available in [BCY+13].

The images are reported as mostly underexposed and of low contrast with strong noise. The authors use them to test state of the art denoising and segmentation algorithms and to develop novel methods in this regard. The data was made publicly available at [Bi15].

The task we want to solve here is different; we see defocused plankton 


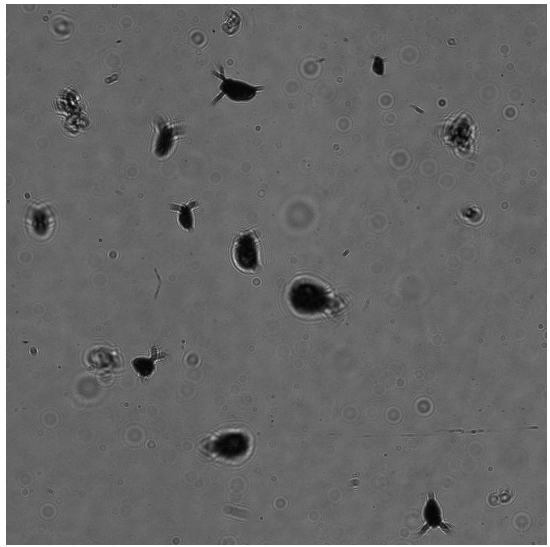

(a) Raw image

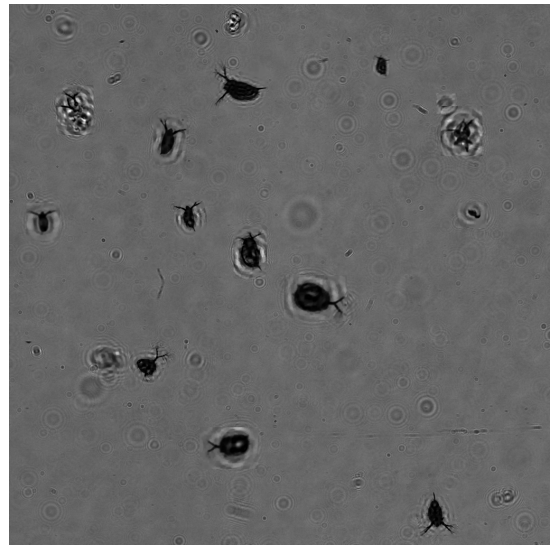

(b) All-in-focus shadowgraph image

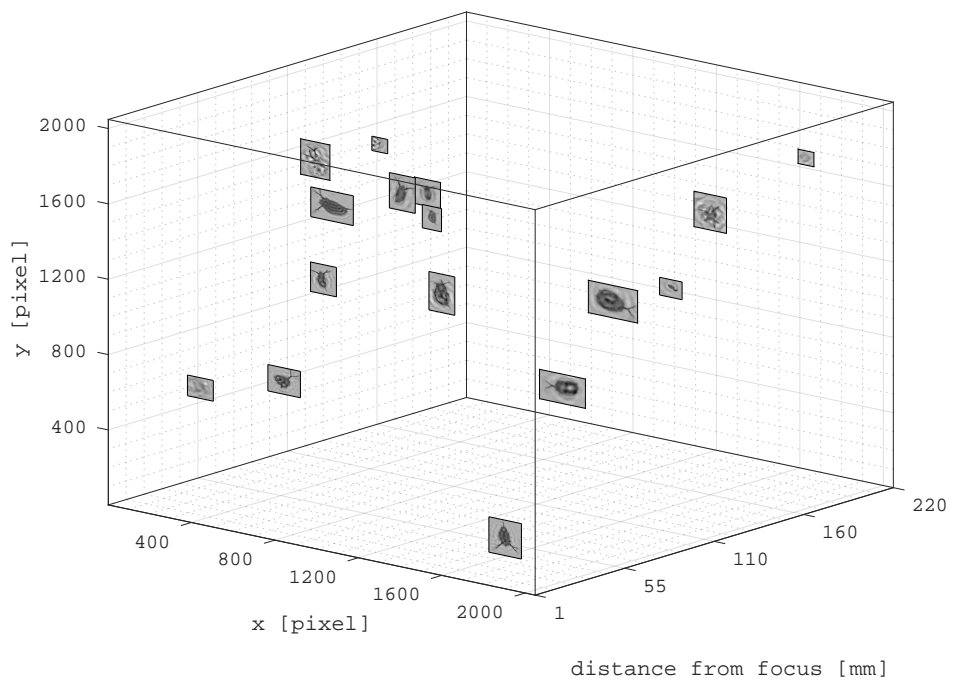

(c) $3 \mathrm{D}$ visualization of depth from defocus

Figure 4.58. All-in-focus and 3D visualization of shadowgraph images. 
4. Applications and Results

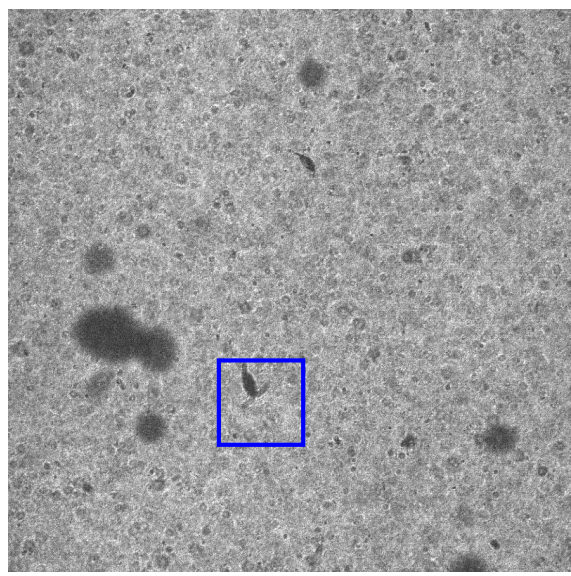

(a)

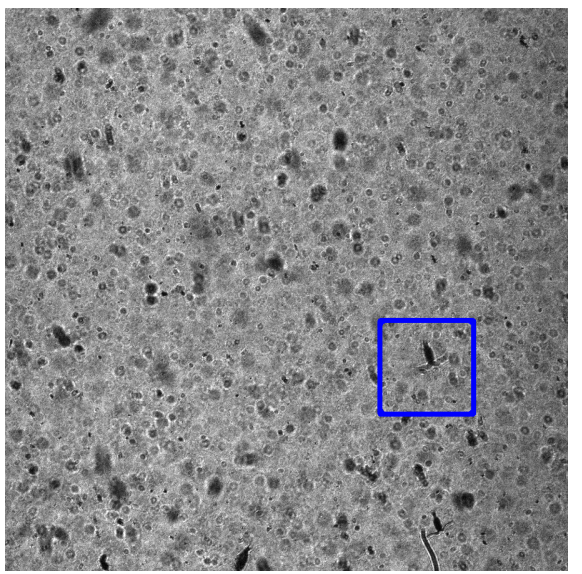

(c)

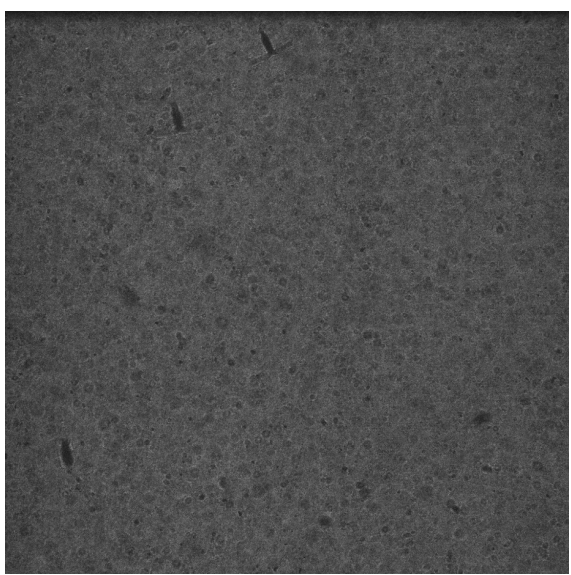

(b)

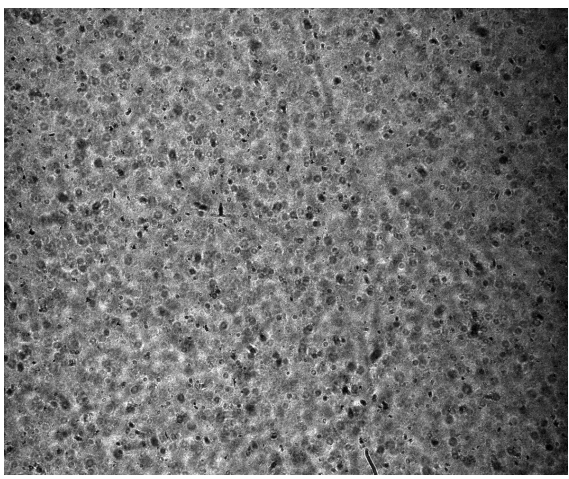

(d)

Figure 4.59. Example images captured with a shadowgraph in Chesapeake Bay from [Bi15]. The blue rectangles highlight the crops used in Figure 4.60. 
and want to test, whether the WFC-restoration algorithms can be applied in this realistic conditions. An example image is shown in Figure 4.59. The images are very noisy and underexposed. In some images such as $4.59 \mathrm{a}$ a pattern can be seen in the background noise, which is probably formed by compression.

We applied the restoration to the provided images with the most noise resistant projective algorithm, the WFC-AP algorithm and the WFC-FISTA algorithm. With the restoration by the WFC-FISTA algorithm some noise is suppressed and the result is not as noisy as the input image. Nevertheless, there is still a lot of turbidity caused by non-plankton objects. The authors of the dataset [BGB+15] used it to test state of the art segmentation algorithms and develop a novel algorithm this is outside the scope of this work. Therefore, the crop segments shown for better visibility of the restoration results where conducted manually.

Results of the restoration and a comparison between the two applied algorithms are shown in Figure 4.60. The images show that even though the in the input images have a very high level of noise, the structure of the plankton can be restored clearly. The difference between the WFCAP and WFC-FISTA algorithm also becomes apparent, as the WFC-FISTA algorithm with its $L_{1}$ regularization contains the noise very well. Inside the cropped segments, even some non-plankton objects are restored, visible as small black dots.

In Figure 4.61 which is a crop of image in Figure 4.59b two objects can be seen. However, cropped objects shown on the left are out of focus and therefore strongly blurred. They are also at different focal distances. On next images the result of the reconstruction is shown, the details are clearly visible. With different correction wavefronts, in the middle image the right plankton is focused, in the right image the left plankton. As can be seen, the images in the Chesapeake Bay were acquired with coherent illumination. 
4. Applications and Results

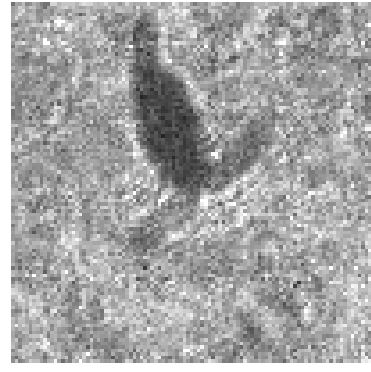

(a) Crop of image 4.59 a

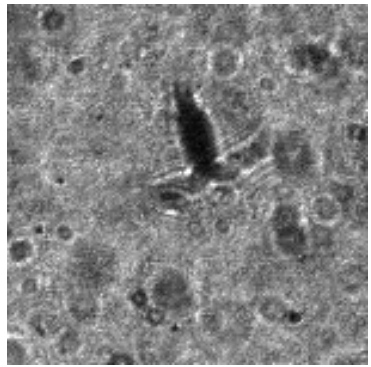

(d) Cropped segment of image $4.59 \mathrm{c}$

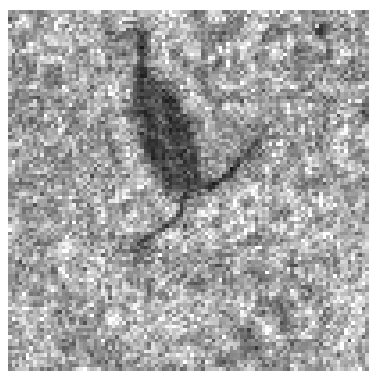

(b) WFC-AP

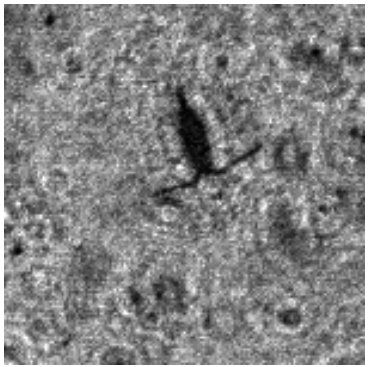

(e) WFC-AP

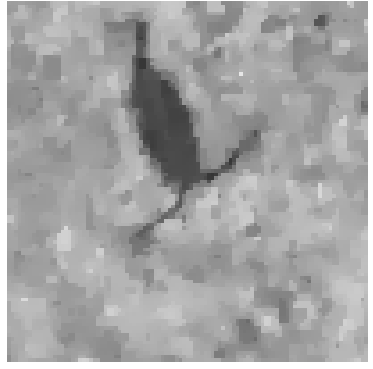

(c) WFC-FISTA

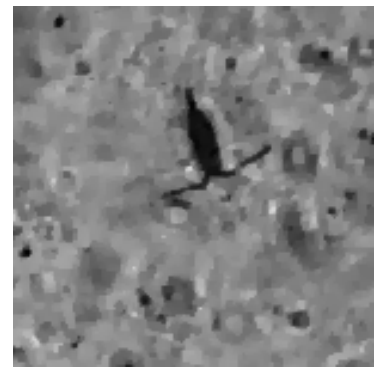

(f) WFC-FISTA

Figure 4.60. Cropped segments of images $4.59 \mathrm{a}$ and $4.59 \mathrm{c}$ with restoration.

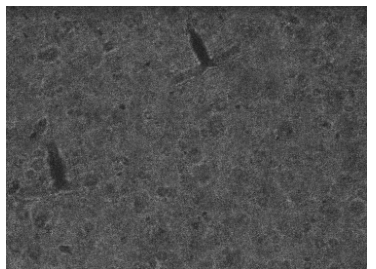

(a) Crop of input image (b) $4.59 \mathrm{~b}$
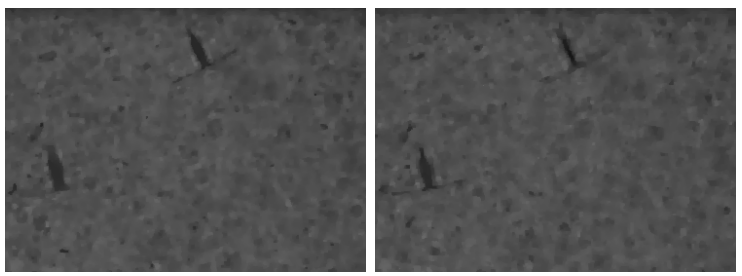

(b) Crop of result image (c) Crop of result image with right plankton sharp with left plankton sharp

Figure 4.61. Dark and noisy input image, refocusing on two plankton objects. 


\section{Chapter 5}

\section{Conclusion}

In this thesis novel algorithm of wavefront correction for coherent image restoration are presented, which are fast, robust and superior to related work (see Figure 4.8, [MKS+09] [KDL13]). The results on both simulated and measured images are very good. The algorithms introduced in this thesis can be summarized in three classes, projective, proximal and projective self-similarity algorithms.

The presented projective algorithms show stable results even in the presence of noise, although theoretically only weak convergence properties can be guaranteed due to the non-convexity of the problem. For the projective algorithms, experiments on simulated images have shown that the WFC-HAAR is the fastest algorithm reaching the best result with the lowest number of iterations. The excellent results of the WFC-HAAR algorithm on simulated images were not confirmed on noisy or real images. The WFC-AP algorithm is very easy to implement and is at the same time most stable against noise and faulty aberration estimates, however it is also the algorithm which requires the most iterations. After extensive evaluation on real images, we would recommend this algorithm over the other projective algorithms, because the good restoration quality outweighs the slight decrease in convergence speed compared to other projective algorithms.

Due to the structure of the WFC-AP algorithm, defined by Fourier transformations and parallel application of constraints, a GPU implementation of this algorithm is possible. Our implementation reaches a very fast runtime of $3.6 \mathrm{~ms}$ per iteration on an image of size $512 \times 512$.

The proximal WFC-FISTA algorithm with total variation regularization delivers superior results on image with imperfections, such as noise and on real images of biological samples. Evaluation and especially comparison 


\section{Conclusion}

with non-regularization methods is difficult because the regularization also has a denoising effect on the sharp image, which negatively impacts the PSNR scores. Visual comparison on real images shows that the results of the WFC-FISTA are superior to the projective algorithms, showing less noise and clearer restoration of edges. The WFC-FISTA algorithm is slower and therefore less suited for real time applications. Our CPU implementation on a $4 \mathrm{GHz}$ processor requires $200 \mathrm{~ms}$ per iteration. If fast real time application is required we recommend the WFC-AP algorithm, else the WFC-FISTA algorithm is clearly the best choice.

For very noisy input images the best results were achieved with the selfsimilarity based WFC-SS algorithm. It should only be used with caution, because on images with less noise, the prior may disturb the image by removing details.

Two fields of application are established in this thesis.

In optical microscopy the algorithm was applied on many different targets with very good results, showing that even very large aberrations can be compensated. The presented good restoration results on real images show that coherent image restoration with wavefront correction can largely extend the depth of field of microscopes. In microscopy typically optics of very high quality and complexity are used. It is the typical assertion that high quality images require aberration free optics. This shows that the algorithm has the potential to bring about large changes in coherent microscopy. The complexity of optical components is in large parts compelled by the need for a high resolution and sharp images, while a microscope with wavefront correction allows using much simpler optics with uncompensated errors and can still deliver good images.

We believe that the success of coherent restoration lies in the nature of coherent imaging, where in contrast to incoherent smeared-blur defects, the coherent ringing-blur defects retain the structural information of the undisturbed object. This makes imperfect optics with larger numerical aperture and size to viable components for coherent microscopy, making it in some applications superior to incoherent imaging, where blur cannot be compensated to such an extend.

The second field of application in this thesis is the shadowgraph. Shadowgraph imaging has numerous applications and we have shown on images of marine plankton how the shadowgraph can profit by the 


\subsection{Future Work}

application of the WFC-algorithm. It effectively increases the depth of field and improves the image quality in areas which are not in total focus. Experiments with post processing show that the artifacts, which restorations with the faster projective WFC-algorithms exhibit, can be very effectively lowered using a chirp filter in the Fourier domain.

We were able to develop a deep learning application based on an artificial neural network to measure the defocus of individual plankton objects and to refocus blurred plankton at different distances from the camera. With this information we gain 3D information of the measurement volume from a single image with a single camera. This can be used for a $3 \mathrm{D}$ visualization of the acquisition volume.

Another result, which we believe is even more important are all-infocus images from volumes with varying strength of blur. This allows the observation of plankton objects in a large volume as if they were planar in a petri dish. Note that the methods were developed for sparse plankton density probes. For high density probes with many individuals per volume such a view does not seem useful, because not all plankton individuals are visible from a single viewpoint.

Moreover, we were able to greatly enhance the image quality of oceanic shadowgraph images by $[\mathrm{BCY}+13]$. The effort taken in the more theoretical parts of this thesis led to the development of very robust restoration algorithms. The Shadowgraph arrangement has a strongly limited depth of field which is caused by physical limits. With the WFC algorithms we have shown that it can be greatly extended. This confirms the relevance of our research.

In conclusion, the novel methods introduced in this thesis have proven to be very useful in practical application.

\subsection{Future Work}

In this section we discuss different avenues of future work as well as possible extensions, applications, as well as links to other fields of research.

Deep learning for post processing After the restoration algorithm has been applied, further post-processing is possible. In this thesis post process- 


\section{Conclusion}

ing using chirp filters is used successfully. Recent works in deep learning, as in [GCB+17] on automatic image enhancement on mobile images, show that post processing with a deep learning approach allow a strong increase in perceived image quality. We believe this could be a way to increase the quality of the restoration, however it may be hard to obtain adequate training data for microscopic or plankton images.

Neural network for defocus estimation In this thesis a neural network for classifying restorations into sharp and unsharp is introduced. A more direct approach is training a regression network, which directly infers the correct wavefront deformation necessary for the compensation. Training a regression network is much harder than training a classification network, because the task it solves is more sophisticated. Furthermore, the very efficient technique demonstrated to generate many training samples for a classification network cannot be used in training a regression network. For a regression network many more objects would have to be labeled by hand.

Faster networks The Alexnet and Googlenet network architectures both show good results. Both networks were designed for image classification with many classes based on visible objects, which means they can recognize many high level features. The task at hand requires only the classification into two classes based on a low level feature, i.e. sharpness or absence of defocus. While simplifying the network might not offer new scientific insights, it should allow speeding up the inference process considerably without loosing relevant classification accuracy.

Multiple wavelengths The current approach discussed in this thesis uses a single wavelength only. While using white light will not work, because it lacks coherence, a collection of images acquired with different wavelengths can be used. Besides potentially allowing restorations of even higher quality or better defocus estimation, the most important advantage of such a multispectral setup is in biological applications. Plankton may be better visible at different colors and additional ultraviolet light may be helpful. 
Note that capturing the same scene multiple times with different wavelengths for more information and a better restoration has already been tested for incoherent light [SFC09].

Live application A microscope typically has a ocular or a camera with a live monitor attached, so that live images can be obtained. We have seen that the WFC algorithms are fast enough to be adapted to such a system, if a slightly lowered quality can be accepted. This is certainly the case for a live preview. Hence, it can be applied on live images. For example, it could be used for real time image restoration in microscopes and either extend the usable depth of field or allow the usage of microscopic lenses with aberrations, which are compensated on the fly. With automatic defocus measurement as we developed for the shadowgraph even fixed focus optics become an option. Note that the full range of the defocus estimation is not required for every frame

As mentioned above, the system described in Chapter 4 reaches $3.6 \mathrm{~ms}$ per iteration of the WFC-AP algorithm on an $512 \times 512$ image. From the results in last chapter we see that an image of reasonable quality, which means that we can recognize details, can be expected with a fixed iterations count of approx. 5. Thus, an live-image could be displayed with a delay $20 \mathrm{~ms}$, which is fast enough for live application on a microscope and could certainly be further improved with some effort in hardware and software.

Adaptive optics Adaptive optics such as deformable mirrors are commonly used in microscopy and astronomy to compensate for optical aberrations, for example in [VMS+11]. A schematic of these systems is shown in Figure 5.1a.

Using a beam splitter, the same wavefront that is observed with a telescope is observed with a wavefront sensor. This sensor measures the deformation of the wavefront. The measurement can now be used to compensate the aberrations with a deformable mirror. The compensated wavefront is aberration free and a sharp image is captured. The system can also be run in a loop, where the mirror is iteratively deformed in such a way that the sensor measures no deformations.

By applying the wavefront correction algorithm, we believe that we can 


\section{Conclusion}

compensate wavefront deformations without a mirror and create sharp images with a much simplified setup, as shown in Figure 5.1b. Our tests with noisy wavefront demonstrate that we should be able to handle the imperfect measurements of wavefront sensors with measurement errors such as $\lambda / 16^{1}$.

Marine micro plastic Marine plastic pollution is an environmental hazard to many species of marine life. The micros plastic particles are either directly part of human debris or they are produced by larger plastic parts being ripped apart by waves [RSW+13]. Especially problematic is the strong stability of plastic particles against other environmental effects and therefore their long lifetime [Roc15]. Many plastics can resist sun and waves for years [RSW+13].

Also especially dangerous, for some parts of marine fauna, are very small particles, which can become part of the seafood chain and cause a large impact on affected species [RHK+13]. Moreover, marine micro plastic can reach even remote and sensitive areas such as the arctic [ZM10].

We believe that the shadowgraph can be used for measuring the concentration of micro plastic particles in the ocean column. The algorithm developed in this thesis should be especially important to expand the possible measurement volume by extending the depth of focus analogous to our experiment on marine plankton. We hope to be able to collaborate with marine scientists to support their research in this regard.

Utilizing the cloud The current implementation uses multithreading on the CPU or a single GPU. Harnessing multiple GPUs should result in a linear speedup and allow even faster results on larger images. Future work could include a cloud service implementation for researchers, which harnesses ad-hoc instances of GPUs from cloud servers. GPU instances are already supported by the Amazon cloud ${ }^{2}$, Microsoft Azure ${ }^{3}$ and Google

\footnotetext{
${ }^{1}$ An example of a wavefront sensor with this accuracy is the Thorlabs WFS1507AR Hartmann-Shack wavefront sensor: https://www. thorlabs. com/thorproduct.cfm?partnumber= WFS150- 7AR

$2_{\text {https : //aws. amazon.com/de/ec2/instance- types/ }}$

${ }^{3}$ https://azure.microsoft.com/en-us/pricing/details/virtual-machines/series/
} 


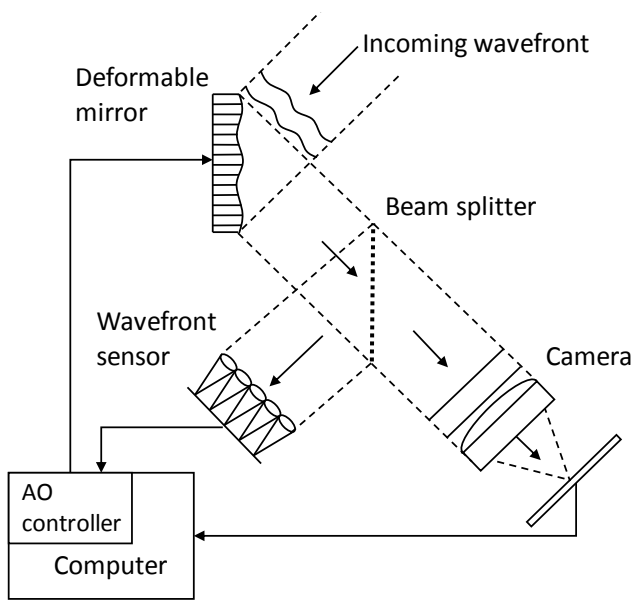

(a) Setup with deformable mirror

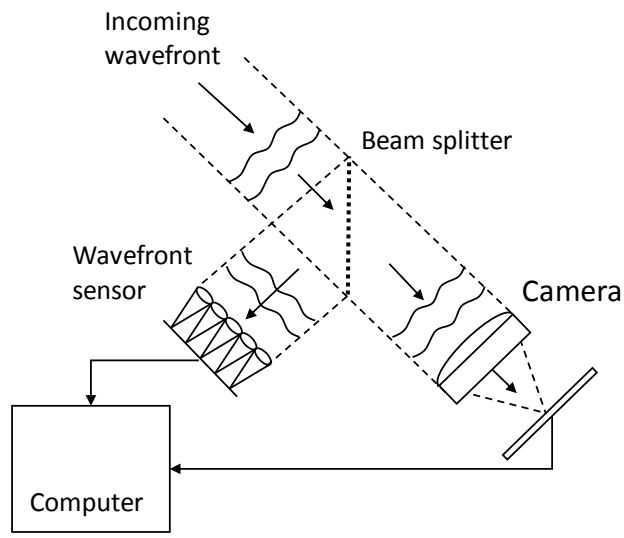

(b) Deformable mirror replaced by novel WFC algorithm

Figure 5.1. Schematic of adaptive optics setup, such as a telescope. An Incoming wavefront is reflected on a deformable mirror, split on a beam splitter, then observed by wavefront sensor and camera. The system is controlled by an acquisition computer and an adaptive optics (AO) controller, compare [Mer88]. 


\section{Conclusion}

Cloud Platform ${ }^{4}$. With cloud computing, hundreds of GPUs can be made available for researchers within seconds (at a price).

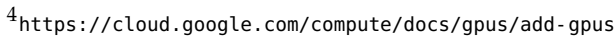


Appendix A

\section{Mathematical and Physical Background}

\section{A.1 Scalar Diffraction Theory}

The propagation of light is an essential part of every imaging system. To explain the propagation of light, this section shall give a condensed introduction to scalar diffraction theory. It is based on [Goo05], [BW80] [RP89] and [Ers06], which give a more detailed introduction into scalar and non-scalar diffraction theory.

The scalar diffraction theory was established as a practical simplification of the rigorous theory of the electromagnetic field.

In this thesis the WFC algorithms are introduced, which use the physical property of phase planarity in a focused image. This introduction concentrates on an complete but condensed explanation with the scalar diffraction theory for this physical condition in the WFC algorithms.

In principle light propagation is governed by three different effects. Refraction is the bending of light on interfaces of optical media with different optical index, which can be described with Snell's law:

$$
n_{1} \sin (\alpha)=n_{2} \sin (\beta),
$$

where $n_{1}$ is the refraction index of medium from which a light ray is incident with angle $\alpha$ from the interface normal at medium $n_{2}$, where the ray is bend to angle $\beta$ from the interface normal.

Reflection causes the direction of an incident ray to be changed at an interface, in such a way that the angle of incidence equals the angle of reflection [BW80].

Both refraction and reflection can be explained by considering light as 
a ray. Together they allow the description of many optical systems with only geometrical formulas. Optics, which can be described solely by these phenomena are called "geometrical optics".

Diffraction is defined as the change of light path, which is not explicable by refraction or reflection [BW80]. It is the part of light propagation which incorporates its wave-like properties.

\section{A.1.1 Huygens-Fresnel Principle}

Thus, to understand diffraction we need to consider light as a wave. Before discussing the specifics of light as an electromagnetic wave, we consider the Huygens-Fresnel principle as a foundation of wave mechanics.

The Huygens-Fresnel principle states that every wave front can be disassembled into a set of secondary waves. The interference of these so called elementary waves in term describes the initial wave front.

\section{A.1.2 Helmholtz Equation}

The electromagnetic properties of light and the interaction between magnetic $H$ and electrical field $E$ with time $t$ are described in the Maxwell equations, without free charge:

$$
\begin{aligned}
\nabla \times E & =-\mu \frac{\partial H}{\partial t} \\
\nabla \times H & =\epsilon \frac{\partial E}{\partial t} \\
\nabla \cdot \epsilon E & =0 \\
\nabla \cdot \mu H & =0,
\end{aligned}
$$

where $\epsilon$ is the electric permittivity and $\mu$ the magnetic permeability of the medium. The permittivity and magnetic permeability of free space is defined as $\epsilon_{0}$ and $\mu_{0}$.

These equations describe the propagation of light waves and with it the spatial and temporal distribution of light and its diffraction patterns accurately. However, an analytical solution of these equations is for practical problems hard, if not impossible [EBY99]. Therefore, we aim for an approximate solution. 
With $B=\mu_{0} H$ the magnetic flux density, from the Maxwell equations it follows that:

$$
\begin{aligned}
\nabla \times \nabla \times E & =-\frac{\partial}{\partial t} \nabla \times B \\
& =-\mu_{0} \epsilon_{0} \frac{\partial^{2} E}{\partial t^{2}}
\end{aligned}
$$

which can be simplified to:

$$
\begin{aligned}
-\mu_{0} \epsilon_{0} \frac{\partial^{2} E}{\partial t^{2}} & =\nabla \times \nabla \times E \\
& =\nabla(\nabla \cdot E)-\nabla^{2} E \\
& =-\nabla^{2} E .
\end{aligned}
$$

This equation, with refractive index $n=\frac{\epsilon}{\epsilon_{0}}$, and speed of light $c=\frac{1}{\sqrt{\mu_{0} \epsilon_{0}}}$ is known as the Helmholtz wave equation of the electrical field:

$$
\nabla^{2} E-\frac{n^{2} \partial^{2} E}{c^{2} \partial t^{2}}=0
$$

This differential equation describes the electric field propagation of a light waves. The wave equation of the magnetic field can be derived analogous.

The electric and magnetic field of light do not need to be considered separately, if the diffraction area and the area of observation are significantly larger than the lights wavelength [RP89]. This approximation is known as the scalar diffraction theory. It simplifies the calculation of diffraction fields, by neglecting the vectorial components of the magnetic and electrical field. A complete vectorial view on diffraction fields can be found in [Soi13].

Combining the electric and magnetic field in a scalar description $u(P, t)$, the scalar Helmholtz equation reads as, for a light wave at position $P$ and time $t$ :

$$
\nabla^{2} u(P, t)-\frac{n^{2} \partial^{2} u(P, t)}{c^{2} \partial t^{2}}=0
$$

A light wave can be described as a harmonic wave with trigonometric functions. For this wave equation, there is a harmonic solution [Ers06] 
with amplitude $a$ and phase $\phi$ at position $P$, which is the basic description of a light wave:

$$
u(P, t)=a(P) \cos (2 \pi v t+\phi(P))=a(P) \cos (k P+\omega t),
$$

where $v$ is the optical frequency,

$$
\omega=2 \pi v
$$

is the angular frequency and

$$
k=\frac{2 \pi}{\lambda}
$$

denotes the wave number.

With phasor $\Phi$ that for a given light wave describes amplitude and phase at spatial position $P$ :

$$
\Phi(P)=a(P) \cdot e^{-j \phi(P)},
$$

$u(P, t)$ can be written with real part Re as:

$$
u(P, t)=\operatorname{Re}\left(\Phi(P) e^{-2 j \pi v}\right) .
$$

Note that the Re function is often omitted and implicitly assumed [Ers06].

Since phasor $\Phi$ is independent of time the scalar Helmholtz equation A.1.12 can be simplified to the Helmholtz equation

$$
\left(\nabla^{2}+k^{2}\right) \Phi=0
$$

From this general description of scalar light waves, the propagation of light distribution is derived using its angular spectrum in the following section.

\section{A.1.3 Propagation of the Angular Spectrum}

The wavefront of a wave is defined as two-dimensional surface formed by those spatial locations, at which the wave has the same phase. With the Huyghens-Fresnel principle, any wavefront can be seen as a composition of elementary waves, which in this three dimensional case, are planar waves. Respecting the phase this can be applied to arbitrary wavefront. Given a monochromatic disturbance a three-dimensional electromagnetic wavefield 
is formed. This composition of elementary waves can be generated using the Fourier transformation, which disassembles the wave-field into individual waves. Using this property, we want to calculate the propagation of a wavefront incident on a measurement plane.

The scalar effects of a wavefield are measured across a plane on which this wave is incident. We define the position of this plane $z=0$, with complex valued measurement of phase and amplitude $U$. On this plane we define a two-dimensional coordinate system and write $U$ as a function of three-dimensional coordinates $(x, y, z)$ and define $A$ by its inverse Fourier transform as the Angular Spectrum:

$$
U(x, y, 0)=\iint A\left(f_{x}, f_{y}, 0\right) e^{j 2 \pi\left(f_{x} x+f_{y} y\right)} \mathrm{d} f_{x} \mathrm{~d} f_{y},
$$

where $f_{x}, f_{y}$ denote the Fourier components or spatial frequencies.

From the complex values of the Fourier transform, the physical wave interpretation can be reached with the observation that the wavefield is composed of a multitude of planar waves described by these complex values.

The wave vector $w$ is defined as:

$$
w=\frac{2 \pi}{\lambda}\left(\begin{array}{l}
\alpha \\
\beta \\
\gamma
\end{array}\right),
$$

where $\alpha, \beta$ and $\gamma$ denote the angles of the propagation direction of a particular planar wavefront.

The angles of $w$ are set by the Fourier components of the angular spectrum with:

$$
\begin{aligned}
& \alpha=f_{x} \lambda \\
& \beta=f_{y} \lambda,
\end{aligned}
$$

and

$$
\begin{aligned}
\gamma & =\sqrt{1-\left(\lambda f_{x}\right)^{2}-\left(\lambda f_{y}\right)^{2}} \\
& =\sqrt{1-\alpha^{2}-\beta^{2}} .
\end{aligned}
$$

A time independent planar wave fulfilling the above conditions is given by:

$$
p(x, y, z)=e^{(j w \cdot r)},
$$


A. Mathematical and Physical Background

with position vector $r \in \mathrm{R}^{3}$. It can be simplified to

$$
\begin{aligned}
p(x, y, z) & =e^{j \frac{2 \pi}{\lambda}(\alpha x+\beta y+\gamma z)} \\
& =e^{j \frac{j \pi}{\lambda}(\alpha x+\beta y)}+e^{j \frac{2 \pi}{\lambda}(\gamma z),}
\end{aligned}
$$

With this notation and the inverse Fourier transform Equation A.1.19 can now be written as

$$
A\left(\frac{\alpha}{\lambda}, \frac{\beta}{\lambda}, 0\right)=\iint U(x, y, 0) e^{-j 2 \pi\left(\frac{\alpha}{\lambda} x+\frac{\beta}{\lambda} y\right)} \mathrm{d} x \mathrm{~d} y,
$$

yielding the angular spectrum at $z=0$.

To calculate the wave-field distribution $U^{\prime}$ with angular spectrum $A^{\prime}$ at a distance $z \neq 0$ from the original wave disturbance $U$ we describe the angular spectrum in direction of the $z$-Axis at $z \neq 0$ as:

$$
A\left(\frac{\alpha}{\lambda}, \frac{\beta}{\lambda}, z\right)=\iint U(x, y, z) e^{-j 2 \pi\left(\frac{\alpha}{\lambda} x+\frac{\beta}{\lambda} y\right)} \mathrm{d} x \mathrm{~d} y .
$$

This equation can also be expressed as:

$$
U(x, y, z)=\iint A\left(\frac{\alpha}{\lambda}, \frac{\beta}{\lambda}, z\right) e^{-j 2 \pi\left(\frac{\alpha}{\lambda} x+\frac{\beta}{\lambda} y\right)} \mathrm{d} \frac{\alpha}{\lambda} \mathrm{d} \frac{\beta}{\lambda} .
$$

The propagation of a wavefront and its angular spectrum can be seen as an interference phenomenon of this individual super-positioned planar waves.

To isolate the propagation term, we regard the Helmholtz equation

$$
\left(\nabla^{2}+k^{2}\right) U=0 .
$$

It must hold for the complex amplitude field $U$ and, because of the linearity of the Fourier Transform, for the angular spectrum $A$ :

$$
\nabla A^{2}+k^{2} A^{2}=0 .
$$

Because we describe the propagation in z-direction with the scalar value of the wave vector in $z$-direction

$$
k_{z}=\frac{2 \pi}{\lambda} \sqrt{1-\alpha^{2}-\beta^{2}},
$$




\section{A.1. Scalar Diffraction Theory}

it follows that:

$$
\frac{\mathrm{d}^{2}}{\mathrm{~d} z^{2}} A\left(\frac{\alpha}{\lambda}, \frac{\beta}{\lambda}, z\right)+k_{z}^{2} \gamma A\left(\frac{\alpha}{\lambda}, \frac{\beta}{\lambda}, z\right)=0 .
$$

A solution to this differential equation can, analogous to the solution of the Helmholtz equation described in Section A.1.2, be given by:

$$
A\left(\frac{\alpha}{\lambda}, \frac{\beta}{\lambda}, z\right)=A\left(\frac{\alpha}{\lambda}, \frac{\beta}{\lambda}, 0\right) e^{j k_{z} z}
$$

The term

$$
\alpha^{2}+\beta^{2}
$$

in $k_{z}$ may attain different values, which have different physical meaning. Three different cases have to be considered:

1. If

$$
\alpha^{2}+\beta^{2}=1
$$

then $k_{z}$ is zero, no propagation in direction of the z-axis occurs and thus we do not need to consider this case further.

2. If

$$
\alpha^{2}+\beta^{2}<1
$$

then $k_{z}$ has an imaginary component. The wave is now called evanescent as it has influence only in the near field. The study of evanescent optical waves is an established independent field of research, see [Fel76], however they can be neglected in the context of optical waves [Goo05]. Therefore, we will not consider this case any further.

3. If

$$
\alpha^{2}+\beta^{2}>1,
$$

the propagation of the wavefront can be seen as a phase shift. This is the default case.

Thus, we can write:

$U(x, y, z)= \begin{cases}\iint A\left(\frac{\alpha}{\lambda}, \frac{\beta}{\lambda}, 0\right) e^{j k_{z} z} e^{j 2 \pi\left(\frac{\alpha}{\lambda} x+\frac{\beta}{\lambda} y\right)} \mathrm{d} \frac{\alpha}{\lambda} \mathrm{d} \frac{\beta}{\lambda} & \text { where } \alpha^{2}+\beta^{2}>1 \\ 0 & \text { else. }\end{cases}$ 
A. Mathematical and Physical Background

With Equation A.1.30 we see that

$$
A\left(\frac{\alpha}{\lambda}, \frac{\beta}{\lambda}, z\right)= \begin{cases}A\left(\frac{\alpha}{\lambda}, \frac{\beta}{\lambda}, 0\right) e^{j k_{z} z} & \text { where } \alpha^{2}+\beta^{2}>1 \\ 0 & \text { else. }\end{cases}
$$

Thereby, the transfer function for the propagation of the angular spectrum in $z$ direction is

$$
H= \begin{cases}e^{j k_{z} z} & \text { where } \alpha^{2}+\beta^{2}>1 \\ 0 & \text { else. }\end{cases}
$$

Substituting variables $k_{z}, \alpha, \beta$ into their definitions above:

$$
H= \begin{cases}e^{j 2 \pi \frac{z}{\lambda} \sqrt{1-\left(\lambda f_{x}\right)^{2}-\left(\lambda f_{y}\right)^{2}}} & \text { where } \sqrt{f_{x}^{2}+f_{y}^{2}}<\frac{1}{\lambda} \\ 0 & \text { else. }\end{cases}
$$

We now have a transfer function for wave propagation in free space, which acts as a linear filter.

We will now simplify this transfer function with the Fresnel approximation. The target of the approximation is the quadratic term of $k_{z}$ :

$$
\begin{aligned}
k_{z} & =\frac{2 \pi}{\lambda} \sqrt{1-\alpha^{2}-\beta^{2}} \\
& =\frac{2 \pi}{\lambda} \sqrt{1-\left(\lambda f_{x}\right)^{2}-\left(\lambda f_{y}\right)^{2}} .
\end{aligned}
$$

With binomial expansion a quadratic term with $\sqrt{1+x}$ can be expanded as (see [Goo05])

$$
\sqrt{1+x}=1+\frac{1}{2} x-\frac{1}{8} x^{2} \ldots
$$

Using only the first terms this leads to the approximation of $k_{z}$ :

$$
\begin{aligned}
k_{z}^{\prime} & \approx \frac{2 \pi}{\lambda}\left(1-\frac{1}{2} \alpha^{2}-\frac{1}{2} \beta^{2}\right) \\
& =\frac{2 \pi}{\lambda}\left(1-\frac{1}{2}\left(\lambda f_{x}\right)^{2}-\frac{1}{2}\left(\lambda f_{y}\right)^{2}\right) \\
& =\frac{2 \pi}{\lambda}-\pi \lambda\left(f_{x}^{2}+f_{y}^{2}\right) \\
& =k-\pi \lambda\left(f_{x}^{2}+f_{y}^{2}\right) .
\end{aligned}
$$


We substitute the approximated $k_{z}^{\prime}$ into Equation A.1.42 and neglect the evanescent waves:

$$
\begin{aligned}
H_{f} & =e^{j k_{z}^{\prime} z} \\
& =e^{j\left(k-\pi \lambda\left(f_{x}^{2}+f_{y}^{2}\right) z\right)} \\
& =e^{j\left(k-\pi \lambda\left(f_{x}^{2}+f_{y}^{2}\right) z\right)} \\
& =e^{j k z} e^{-j \pi \lambda z\left(f_{x}^{2}+f_{y}^{2}\right)},
\end{aligned}
$$

which is the transfer function of the Fresnel approximation. Disregarding constant phase delay this is:

$$
H_{f}=e^{-j \pi \lambda z\left(f_{x}^{2}+f_{y}^{2}\right)},
$$

This approximation assumes that the angles $\alpha$ and $\beta$ are small and is valid only in the near field [RP89]. This is the same approximation made in paraxial geometric optics [Goo05]. The inverse Fourier transform of $H_{f}$ is called the Fresnel diffraction impulse response:

$$
h_{f}=\frac{e^{j k z}}{j \lambda z} e^{j \frac{\pi}{\lambda z}\left(x^{2}+y^{2}\right)} .
$$

or without constant phase delay:

$$
h_{f}=\frac{1}{j \lambda z} e^{j \frac{\pi}{\lambda z}\left(x^{2}+y^{2}\right)} .
$$

\section{A.1.4 Influence of a Lens}

In the previous section the propagation of a wavefront in free space with Fresnel approximation was derived. In this section this topic is revisited, however now the focus lies on the influence of a lens.

The difference in optical index between two media, is equivalent to the difference in light speed between them. Due to the difference in optical index between glass and air, a phase shift in the wavefront and therefore its angular spectrum is introduced. This phase shift is dependent on the optical path length difference which is induced by both lens thickness and refractive index. A typical lens can be seen as a composition of one or two spherical surfaces and a planar part in between [Goo05], as drawn in 
A. Mathematical and Physical Background

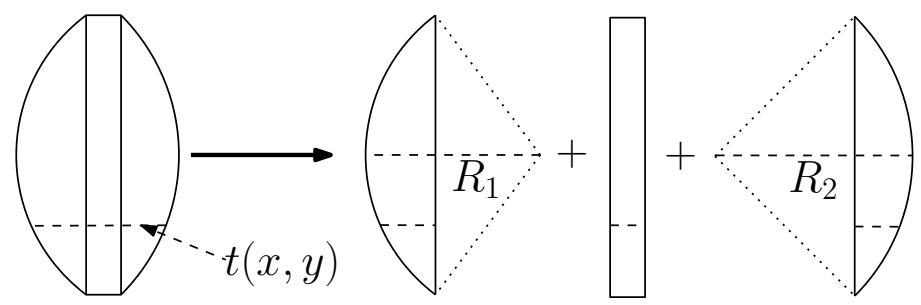

Figure A.1. A spherical thick lens can be seen as a composition of three parts, adaptation from [Ers06].

Figure A.1.

Such a lens has a focal length defined by [BW80]:

$$
f=\frac{1}{(n-1)\left(\frac{1}{R_{1}}-\frac{1}{R_{2}}\right)},
$$

where $n$ denotes the optical index of the glass and $R_{1}, R_{2}$ the radii of the spherical surfaces.

The thickness function $t(x, y)$ at location $(x, y)$ is derived in [Goo05] and [Ers06] and can be seen as the addition of the thickness function of the three components, where $t_{0}$ is the maximum thickness of the lens:

$$
t(x, y)=t_{0}-R_{1}\left(1-\sqrt{1-\frac{x^{2}+y^{2}}{R_{1}^{2}}}\right)+R_{2}\left(1-\sqrt{1-\frac{x^{2}+z^{2}}{R_{2}^{2}}}\right) .
$$

Note that the radii may also be negative, resulting in a concave surface.

With the paraxial approximation, that is assuming that the light is incident in small angles from the optical axis, this equation is simplified into:

$$
t(x, y)=t_{0}-\frac{x^{2}+y^{2}}{2}\left(\frac{1}{R_{1}}-\frac{1}{R_{2}}\right) .
$$

With its higher optical index, the thickness of the lens induces a phase transformation, which with Equation A.1.58 is

$$
t(x, y)=e^{j k t_{0}} e^{-j \frac{k}{2 f}\left(x^{2}+y^{2}\right)}
$$




\section{A.2. Wavefront Focusing}

or without constant phase delays:

$$
t(x, y)=e^{-j \frac{k}{2 f}\left(x^{2}+y^{2}\right)} .
$$

\section{A.2 Wavefront Focusing}

Because of its high relevance to the algorithms introduced in this thesis, we will now consider the focusing or defocusing effect of a lens on an incoming wavefront. The results of this calculations are used in Section 3.1 to motivate the novel WFC-algorithms.

An undisturbed wavefront $U$ for example from an microscopic LED illumination shines through an object, which means that it is modulated with the transmittance function of an object $t_{0}$. After a distance the light occurs on a lens, with incident wavefront distribution $U_{\text {in }}$ and wavefront distribution $U_{l}$ behind the lens. With the lens this light is then focused on a focal plane with wavefront distribution $U_{f}$. Figure A.2 gives an overview of the setting.

Thus, we have a wavefront $U_{0}$ modulated by the object:

$$
U_{o}(x, y)=U(x, y) t_{o}(x, y) .
$$

With the propagation of the angular spectrum, we know that:

$$
\mathcal{F}\left(U_{l}(x, y)\right)=\mathcal{F}\left(U_{o}(x, y)\right) H_{f} .
$$

Furthermore, we have the influence of the lens on the incoming wavefront to consider. Let the incoming wavefront distribution at location $(x, y)$ of the lens be $U_{i n}$. Behind the lens the lens distribution $U_{l}$ with lens phase shift in Equation A.1.62 is:

$$
U_{l}(x, y)=U_{i n}(x, y) e^{-j \frac{k}{2 f}\left(x^{2}+y^{2}\right)} .
$$

As the next step, we are interested in the distribution at the focal plane, hence we set $z$-coordinate of the wavefront propagation to the focal length $f$, which means directly at the focal plane. With the filter kernel of the Fresnel wavefront propagation $h_{f}$ A.1.57, the target distribution $U_{z=f}(s, t)$ 
A. Mathematical and Physical Background

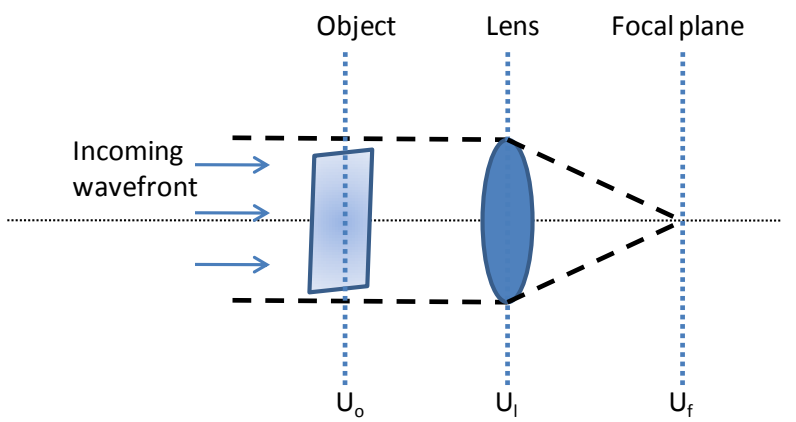

Figure A.2. An incoming wavefront is incident on a semitransparent object. The propagated wavefront is focused with a lens into the focal plane, adapted from [Goo05].

at location $(s, t)$ is

$$
\begin{aligned}
U_{z=f}(s, t) & =U_{l}(x, y) \otimes h_{f} \\
& =U_{l}(x, y) \otimes \frac{e^{j k z}}{j \lambda z} e^{j \frac{\pi}{\lambda z}\left(x^{2}+y^{2}\right)} .
\end{aligned}
$$

This convolution can be expanded, see Fourier transform properties in [Boa03]:

$$
U_{z=f}(s, t)=\frac{e^{j k z}}{j \lambda z} \iint U_{l}(x, y) e^{j \frac{\pi}{\lambda z}(s-x)^{2}+(t-y)^{2}} \mathrm{~d} x \mathrm{~d} y
$$

and constant factors can be moved:

$$
U_{z=f}(s, t)=\frac{e^{j k z}}{j \lambda z} e^{j \frac{\pi}{\lambda z}\left(s^{2}+t^{2}\right)} \iint U_{l}\left(f_{x}, f_{y}\right) e^{j \frac{\pi}{\lambda z}\left(x^{2}+y^{2}\right)} e^{-j \frac{-j 2 \pi}{\lambda z}(x s+y t)} \mathrm{d} x \mathrm{~d} y,
$$


and dropped:

$$
U_{z=f}(s, t)=\frac{1}{j \lambda z} e^{j \frac{\pi}{\lambda z}\left(s^{2}+t^{2}\right)} \iint U_{l}(x, y) e^{j \frac{\pi}{\lambda z}\left(x^{2}+y^{2}\right)} e^{-j \frac{-j 2 \pi}{\lambda z}(x s+y t)} \mathrm{d} x \mathrm{~d} y .
$$

The integral part of this equation is equivalent to the Fourier transform of $U_{l}$ multiplied with the propagation term in Equation A.1.55, which is the Fourier transform of the propagated wave without lens influence. The coordinates of the focal plane $(s, t)$ are defined by the Fourier components [Goo05] of the Fourier transform of $U_{l}$ with:

$$
\begin{aligned}
& x=\frac{s}{\lambda f} \\
& y=\frac{t}{\lambda f} .
\end{aligned}
$$

Thereby with $H_{f}$ from Equation A.1.55:

$$
U_{z=f}(s, t)=\frac{1}{j \lambda z} e^{j \frac{\pi}{\lambda z}\left(s^{2}+t^{2}\right)} \mathcal{F}\left(U_{o}\right)\left(\frac{s}{\lambda f}, \frac{t}{\lambda f}\right) H_{f}\left(\frac{s}{\lambda f}, \frac{t}{\lambda f}\right) .
$$

Now we insert the incoming wavefront of the lens $U_{l}$ as the propagated wavefront from the object at distance $d$ with Equation A.2.2:

$$
U_{z=f}(s, t)=\frac{1}{j \lambda z} e^{j \frac{\pi}{\lambda z}\left(s^{2}+t^{2}\right)} \mathcal{F}\left(U_{o}\right)\left(\frac{s}{\lambda f}, \frac{t}{\lambda f}\right) H_{f}\left(\frac{s}{\lambda f}, \frac{t}{\lambda f}\right) .
$$

With the definition of $H_{f}$ :

$$
U_{z=f}(s, t)=\frac{1}{j \lambda z} e^{j \frac{\pi}{\lambda z}\left(s^{2}+t^{2}\right)} e^{-j \pi \lambda d\left(\left(\frac{s}{\lambda f}\right)^{2}+\left(\frac{t}{\lambda f}\right)^{2}\right)} \mathcal{F}\left(U_{0}\right)\left(\frac{s}{\lambda f}, \frac{t}{\lambda f}\right),
$$

and sorting of the prefactors, as well as substituting $z$ with $f$

$$
\begin{aligned}
U_{z=f}(s, t) & =\frac{e^{j \frac{\pi}{\lambda} \frac{1}{f}\left(s^{2}+t^{2}\right)-j \frac{\pi}{\lambda} \frac{d}{f^{2}}\left(s^{2}+t^{2}\right)}}{\lambda j f} \mathcal{F}\left(U_{o}\right)\left(\frac{s}{\lambda f}, \frac{t}{\lambda f}\right) \\
& =\frac{e^{j \frac{\pi}{\lambda} \frac{1}{f}\left(s^{2}+t^{2}\right)-j \frac{\pi}{\lambda} \frac{d}{f^{2}}\left(s^{2}+t^{2}\right)}}{\lambda j z} \mathcal{F}\left(U_{o}\right)\left(\frac{s}{\lambda f}, \frac{t}{\lambda f}\right),
\end{aligned}
$$


A. Mathematical and Physical Background

we can simplify to:

$$
U_{z=f}(s, t)=\frac{e^{j \frac{\pi}{\lambda f}\left(1-\frac{d}{f}\right)\left(s^{2}+t^{2}\right)}}{\lambda j f} \mathcal{F}\left(U_{o}\right)\left(\frac{s}{\lambda f}, \frac{t}{\lambda f}\right) .
$$

In the focus of a lens $d=f$, the phase term is zero, which means that the phase shift of the lens and the wavefront propagation negate each other. The transform between aperture and focus in a lens is the Fourier transform and there are no additional phase terms, meaning that if the incoming wavefront is planar, so is the focal plane wavefront. This is the physical motivation for the planar phase condition of the WFC-algorithms.

In contrast, if the system is not in focus, then the spherical aberration factor remains. This defocus factor and other optical errors can be described elegantly using Zernike polynomials, which are introduced in Section A.5. 


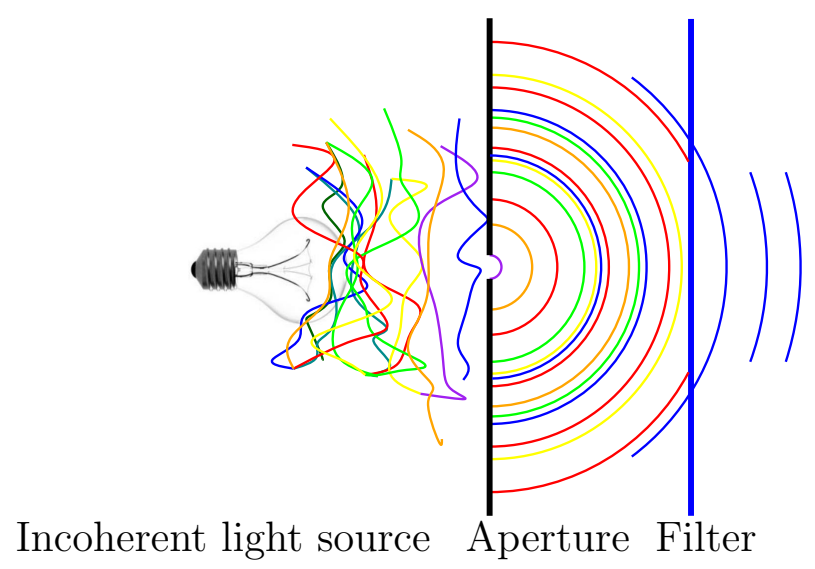

Figure A.3. Spatially and temporally coherent light from an incoherent light source $^{1}$.

\section{A.3 Coherence}

The topic of optical coherence is very important to this thesis, which is the restoration of coherent images. In the introduction chapter, the differences between coherent and incoherent image formation are presented, in which knowledge about coherence is assumed. In this section we want to give a detailed overview of the phenomenon of coherence and provide the reader with a good understanding of it.

In general, two aspects of coherence can be distinguished: Spatial coherence and temporal coherence. We will first give a visual example and then discuss both aspects in more detail in the following paragraphs .

In Figure A.3 a very incoherent light source, a light bulb is placed in front of a small aperture. This aperture restrict the light waves of the light source, acting as a spatial filter. The light behind this aperture is spatially coherent. Next, this light occurs on a bandwidth color filter, which lets only light with a certain frequency in this instance blue light pass. Behind this frequency filter, the light is both spatially and temporally coherent. In short, spatial coherence is about the extend of the light source and

\footnotetext{
${ }^{1}$ Drawing inspired by William Beaty: http://amasci.com/miscon/coherenc.html
} 


\section{A. Mathematical and Physical Background}

temporal coherence is about the bandwidth of the light waves, i.e. how monochromatic their light is.

Temporal coherence Temporal coherence describes how 'temporally', which means over time at a given place, coherence or (using a different word) correlation between the waves occurs in a wavefield. The scalar diffraction theory yields that an optical wave can be expressed as (see Equation A.1.17):

$$
u(P, t)=\operatorname{Re}\left(U(P) e^{-2 j \pi v t}\right) .
$$

Clearly, in a wavefield consisting of a single pure wave of one frequency, for a given $P$ there exists a very strong relationship between two times $t$, because the wave is periodic with $2 \pi$. Two waves, which have the same frequency $v$, have the same angular velocity $2 \pi v$ and hence there is a temporal coherence between these waves and they can interfere with each other.

Temporal coherence is the result of a narrow spectrum of light and can therefore be measure for the breadth of the spectrum of light. A small breadth means a high temporal coherence, a broad spectrum means a low frequency coherence. High temporal coherence means that light is monochromatic. The color of light is typically described by the wavelength. Because frequency and wavelength of light are connected by the following relation,

$$
\lambda=\frac{c}{v^{\prime}},
$$

with speed of light in vacuum $c=3 \cdot 10^{8} \frac{\mathrm{m}}{\mathrm{s}}$, the term wavelength coherence can also be used.

Temporal coherence is measured by the coherence length, which describes the distance which the light travels, while the waves can interfere with each other. The coherence length dependent on the breath of the frequency spectrum $\delta_{f}$ is defined as [Pas08]:

$$
L_{c o h}=\frac{c}{\pi \delta_{f}}
$$

the coherence time can be easily derived from the coherence length with:

$$
t_{c o h}=\frac{L_{c o h}}{c} .
$$


Spatial coherence Spatial coherence is similarly defined to temporal coherence. The difference is that now the phase between two spatially distinct points on a plane perpendicular to the propagation direction is examined at the same time. If there is a correlation between the phase at these points, then these waves can interfere and spatial coherence is fulfilled.

Typically, this is accomplished using a small light source or a limited aperture. If compared to the distance, the light source is very small, the light waves will propagate almost in parallel, which means that at two points orthogonal to the direction of propagation of a wave the difference in phase is small, up to a constant factor [Ers06]. Important is the angular source size of the illumination.

In Figure A. 4 two points $x_{1}$ and $x_{2}$ are located perpendicular to the direction or propagation of a light wave with a distance of $d_{x}$ between them and a distance of $d_{x} / 2$ from the optical axis and center point $x_{m}$. They are illuminated through an aperture of size $d_{s c}$.

Clearly, the optical path between the upper point of the aperture and $x_{1}$ is shorter than the optical path from the lower point of the aperture. The opposite is true for $x_{2}$. Dependent on the extend of this effect, This difference in optical length may result in the waves being in phase at $x_{1}$ are out of phase in $x_{2}$. This is a result of the angular source size. [Bre17] defines $d_{\max -s c}$ as the maximum size for an aperture of spatially coherent light for points at distance $L$ from the aperture and $d_{x}$ from the axis:

$$
d_{\max -s c}=0.16 \lambda \frac{L}{d_{x}}
$$

Another formula is given in [RP89], spatial coherence demands that all optical path length differences in an optical system $\delta_{L}$ are much smaller than the fraction between speed of light and breath of the frequency spectrum:

$$
\delta_{L} \ll \frac{c}{\delta_{f}} .
$$

With spatial coherence between two points, the coherence area is defined as the area in which the phase of a propagating wave is in sync. The axial coherence length is defined above by the temporal coherence and the coherence area is defined by spatial coherence. Combined this results in a 
A. Mathematical and Physical Background

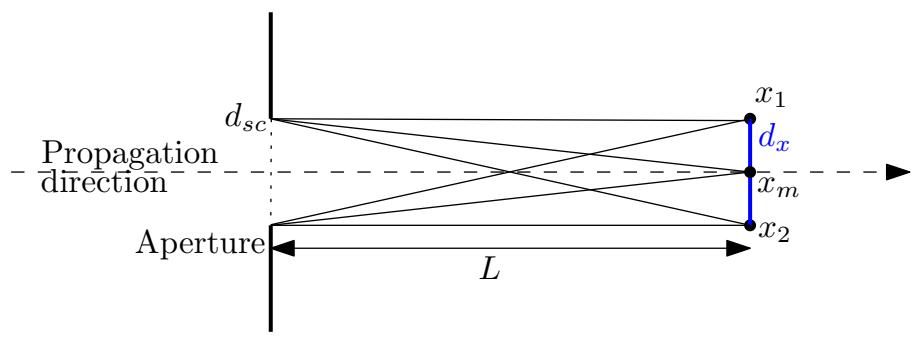

Figure A.4. Illustration for spatial coherence calculations. Adapted from: [RP89][Bre17].

coherence volume [RP89].

Based on coherence, light sources can be categorized. Examples of light sources with high temporal coherence are monochromatic LEDs, lasers and monochromatic florescent lamps. High spatial coherence can be found in lasers, small LEDs and, because of their large distance to the observer, stars. 


\section{A.4 Peak Signal to Noise Ratio}

Throughout this thesis the peak signal to noise ratio (PSNR) is used as a measure of restoration quality, i.e. how similar the restored image signal $g l s s: i r$ and the reference image signal $I_{i n c}$ are. The most used measure of restoration quality in image processing is the peak signal noise ratio (PSNR) [HG08]. It has the strong advantage that in contrast to other popular measures such as the structural similarity measurement index (SSMI) [WBS+04], that it is independent of the human perception model and describes the difference between two image with an objective, technical measure [HZ10]. It is defined as

$$
P S N R=10 \log _{10}\left(\frac{\max ^{2}}{M S E}\right) d B,
$$

where $\max$ is the maximum value the image intensity can attain and MSE denotes the mean squared error. In floating point images used in throughout this thesis this value is 1.0. For restored and reference images $I_{r}$ and $I_{i n c}$ of size $h \times w$, the MSE is calculated by:

$$
M S E=\sum_{j=1}^{h} \sum_{i=1}^{w}|R(i, j)-S(i, j)|^{2} .
$$




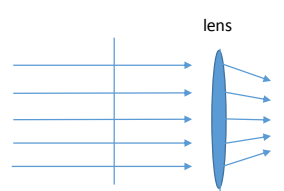

(a) Planar and spherical wavefront

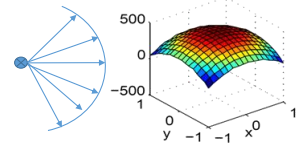

(b) Spherical wavefront

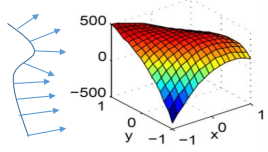

(c) Arbirary wavefront

Figure A.5. Visualization of wavefront shapes.

\section{A.5 Zernike Polynomials}

A wavefront is defined as the set of points of equal phase. Such a wavefront can occur in various shapes. An illustration is given in Figure A.5. Parallel light has a planar wavefront. Superimposing the phase shift of a lens a spherical wavefront is gained. A spherical wavefront can also be visualized either by arrows showing the direction of propagation perpendicular to the points of equal phase or in the three dimensional case by drawing the phase shift of such a set of points across the $x-y$ plane of propagation. The same can be applied to arbitrary wavefront, as Figure A.5 shows. An concise way to describe wavefront deformations are the Zernike polynomials shown in Table A.1.

Zernike polynomials form an orthogonal basis [Nol76] on a unit circle as in Figure A.6 and can thus describe any wavefront deformation. We will use the normalization of the Zernike polynomials as used in [Nol76], which we will use for the scope of this thesis. Note that some prior publications such as [BW80] follow a different definition of the Zernike polynomials than given, caused by a different choice of normalization.

In polar coordinates with angle $\theta$, radial distance $p$, variables $m, n \in$ $\mathrm{N}, m \neq 0, n \geqslant m$ the even Zernike polynomial is defined as [Nol76]:

$$
Z_{n}^{m}(p, \theta)=\sqrt{n+1} R_{n}^{m}(p) \sqrt{2} \cos (m \theta),
$$

while the odd Zernike polynomial is defined as:

$$
Z_{n}^{-m}(p, \theta)=\sqrt{n+1} R_{n}^{m}(p) \sqrt{2} \sin (m \theta) .
$$

This definition of th Zernike polynomials is built on the radial polynomial 
A.5. Zernike Polynomials

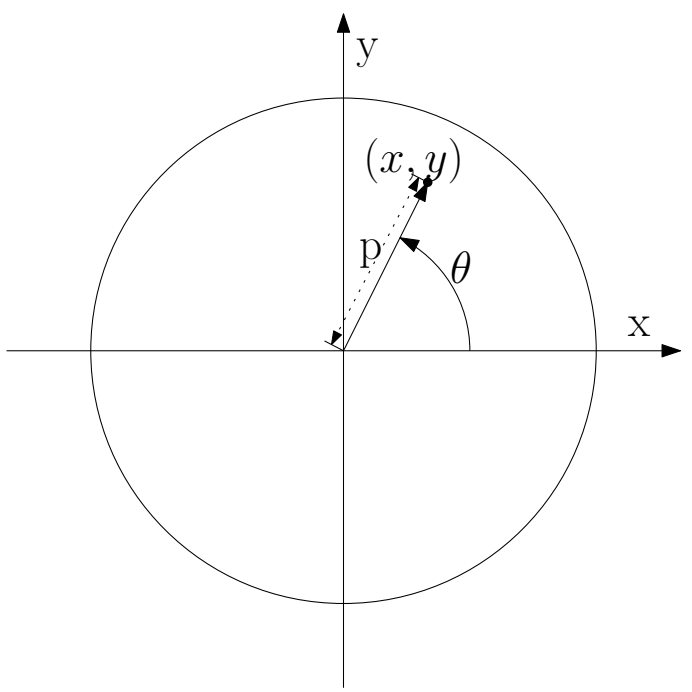

Figure A.6. Definition of $\mathrm{p}$ and $\theta$ for the Zernike polynomial (adapted from [GW06]).

$R$. The variables $n$ and $m$ may be seen as radial degree and azimuthal frequency [Nol76]. If $n-m$ is even, the radial polynomial $R$ is defined by [BW80]:

$$
R_{n}^{m}(p)=\sum_{s=0}^{\frac{n-m}{2}} \frac{(-1)^{s}-(n-s) !}{s !\left(\frac{n+m}{2}-s\right) !\left(\frac{n-m}{2}-s\right) !} p^{n-2 s},
$$

otherwise by

$$
R_{n}^{m}(p)=0 .
$$

Typically, the Zernike polynomials are visualized as if they were deformations of circular wavefronts. In Figure A.7 we show such a color coded visualization of the Zernike polynomials, where high values are shown in blue and low values in yellow. The polynomials are ordered in a pyramid by their radial degree $n$. The defocus aberration, see Table A.7, is shown in the third row, center column.

Besides using double indices for radial degree and azimuthal frequency there exists another ordering of Zernike polynomials following [Nol76]. 


\section{A. Mathematical and Physical Background}

This so called Noll index allows a strict ascending ordering of Zernike polynomials, denoted $Z$ with a single index. This mapping between Noll index, mode and the classic Seidel aberrations, such as astigmatism and coma is shown in Table A.1.

Table A.1. Zernike polynomials with mode index by [Nol76] showing the relationship with Seidel wavefront aberrations.

\begin{tabular}{llll}
\hline Noll index & Mode & Polynomial & Seidel aberration \\
\hline$Z_{1}$ & $Z_{0}^{0}$ & 1 & \\
$Z_{2}$ & $Z_{1}^{1}$ & $2 p \sin \theta$ & Tilt - horizontal \\
$Z_{3}$ & $Z_{1}^{-1}$ & $2 p \cos \theta$ & Tilt - vertical \\
$Z_{4}$ & $Z_{2}^{0}$ & $\sqrt{3}\left(2 p^{2}-1\right)$ & Defocus \\
$Z_{5}$ & $Z_{2}^{-2}$ & $\sqrt{6} p^{2} \sin 2 \theta$ & Astigmatism - horizontal \\
$Z_{6}$ & $Z_{2}^{2}$ & $\sqrt{6} p^{2} \cos 2 \theta$ & Astigmatism - vertical \\
$Z_{7}$ & $Z_{3}^{-1}$ & $\sqrt{8}\left(3 p^{3}-2 p\right) \sin \theta$ & Coma (3rd order) \\
$Z_{8}$ & $Z_{3}^{1}$ & $\sqrt{8}\left(3 p^{3}-2 p \cos \theta\right.$ & Coma (3rd order) \\
\hline
\end{tabular}


A.5. Zernike Polynomials
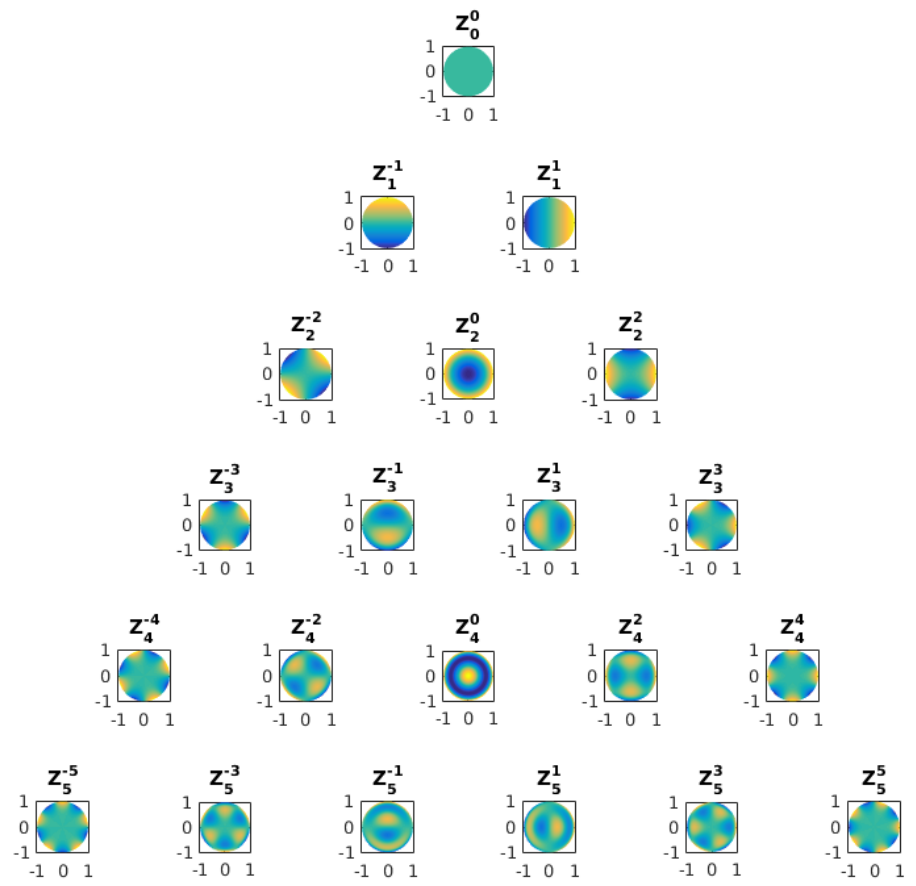

Figure A.7. Color-coded visualization of the Zernike polynomials in a pyramid with high values in blue and low values in yellow. Created with Matlab code by [TSS11] and [STS+13]. 


\section{A.6 Projections onto Convex Sets}

Projections onto convex sets (POCS) is a common approach to solving feasibility problems. Given a Hilbert space $H$ with norm $\|\ldots\|$ and inner dot product, let $a$ and $b$ be two points in $S \subset H$, then the set $S$ is convex if

$$
a \cdot t+b \cdot(1-t) \in S, t \in[0 . .1]
$$

and therefore any convex combination of $a$ and $b$ is also in $S$. In other words, a set is convex, if any linear combination of two points from the set is inside the set.

For $t \in H$ and $S \subset H$, let the result of a projection $P_{S}$ onto set $S$ be defined as

$$
p=\operatorname{argmin}_{s \in S}\|s-t\|=P_{S}(t) .
$$

In the following this introduction is limited to finite dimensional Hilbert spaces. Given two convex sets $A \subset H$ and $B \subset H$ and their nonempty intersection $C=A \cap B$. We define a sequence of iterations starting with initial value $y_{1}$ set to an arbitrary value $x$. This sequence is indexed with $n \in \mathbb{N}$, so that the $\mathrm{n}$-th element is denoted by $y_{n}$. The von-Neumann algorithm reaches a new iterative by alternating application of projections onto these sets:

$$
y_{n+1}=P_{A} P_{B}\left(y_{n}\right) \text {. }
$$

This algorithm is therefore also called $\mathrm{MAP}$ (multiple alternating projections), see [ER11].

The averaged projections algorithm projects the current iterate with both projectors and uses the average for the next iteration:

$$
y_{i+1}(n)=\frac{1}{2}\left(P_{B}\left(y_{i}(n)\right)+P_{A}\left(y_{i}(n)\right)\right) .
$$

The reflector operator

$$
R=2 P-I
$$

consists of one application of the projection, but executes the motion of the projector twice, where $P$ is a given projector and $I$ is the identity operator [Mar07a].

In [Hau68] Haugazeau presents a strongly convergent algorithm for finding the intersection of two convex sets. Based on his works Bauschke et. al. define the Haugazeau-like average alternating reflections algorithm 
(HAAR) [BCL06].

Let $H$ be a real Hilbert space and let $(x, y, z) \in H^{3}$ be a tuple, with the following property

$$
\begin{array}{r}
w \in H \mid(x-y) \cdot(x-y) \leqslant 0 \\
\cap w \in H \mid(w-z) \cdot(y-z) \leqslant 0 \neq \varnothing .
\end{array}
$$

Then define the following intermediate variables

$$
\begin{aligned}
& \pi=(x-y) \cdot(y-z) \\
& \mu=\|x-y\|^{2} \\
& \nu=\|y-z\|^{2} \\
& \rho=\mu \nu-\pi^{2} .
\end{aligned}
$$

Also let

$$
\begin{aligned}
& Q(x, y, z) \\
& \quad= \begin{cases}z, & p=0 \text { and } \pi \geqslant 0 \\
x+\left(1+\frac{\pi}{v}\right)(z-y), & \rho>0 \text { and } \pi v \leqslant \rho \\
y+\left(\frac{v}{\rho}(\pi(x-y)+\mu(z-y)),\right. & \rho>0 \text { and } \pi v<\rho .\end{cases}
\end{aligned}
$$

be an helper operator [Hau68].

In [Hau68] Haugazeau defines the sequence $\left(y_{n}\right)_{n \in \mathbb{N}}$ as

$$
y_{n+1}=Q\left(x, Q\left(x, y_{n}, P_{B} y_{n}\right), P_{A} Q\left(x, y_{n}, P_{B} y_{n}\right)\right)
$$

and proves it convergences strongly to a point $P_{C} x$ in the intersection $C$. In [BCL06] Bauschke et al. improve on this recursive algorithm and establish the HAAR algorithm. With operator $T$ defined as

$$
T=\frac{1}{2} R_{B} R_{A}+\frac{1}{2}
$$

the iteration formula is

$$
y_{n+1}=Q\left(x, y_{n},\left(1-\mu_{n}\right) y_{n}+\mu_{n} T y_{n}\right),
$$

where $\left(\mu_{n}\right)_{n \in \mathbb{N}}$ is an arbitrary sequence of values in $\left.] 0,1\right]$ with $\inf _{n \in \mathbb{N}} \mu_{n}>$ 0 . 
A. Mathematical and Physical Background

\section{A.7 FISTA}

ISTA (iterative shrinkage thresholding algorithm) is an optimization algorithm typically employed for solving linear inverse problem [LM79] [BT09a] . Such a linear inverse problem can be described by

$$
A x=b,
$$

where matrix $A \in \mathrm{R}^{m \times n}$ describes a linear operator, $b \in R^{m}$ is a known signal and $x \in R^{n}$ is the target undisturbed signal, which is to be reconstructed from $b$.

If for example $A$ describes a blur operator, then this problem is a deblurring or deconvolution problem [BT09a].

The standard approach is to use a least squares approach, which results in the following minimization problem:

$$
\operatorname{argmin}_{x}\|A x-b\|^{2} \text {. }
$$

Because this kind of approach is in many cases, such as in deconvolution[LWD+09], ill-conditioned, regularization is added to guide to optimization to more likely solutions. A popular choice for regularization in image restoration is the $l_{1}$ regularization, due to its ability to closely match the natural distribution of gradients in an image [LFD+07], thus we minimize the following term:

$$
\operatorname{argmin}_{x}|| A x-b||^{2}+\lambda|x|_{1},
$$

where $\|_{1}$ denotes the $l_{1}$ norm and $\lambda \in \mathrm{R}$ a weighting factor to control the strength of the regularization.

One can recognize that this inverse linear problem fits the following structure

$$
\operatorname{argmin}_{x} f(x)+g(x),
$$

where function $f: \mathrm{R}^{n} \rightarrow \mathrm{R}$ is a convex function and $C^{1,1}$ smooth and function $g$ defined as $g: \mathrm{R}^{n} \rightarrow \mathrm{R}$ continuous and convex. Furthermore this problem must have a non-empty solution set. The quadratic data term fits the description of $f$, while the $l_{1}$ regularization perfectly fits the description of $g$.

Following [BT09a] ISTA relies on the quadratic approximation of (A.7.4) 
at point $x^{\prime}$ for a $x \in \mathrm{x}^{2}$ :

$$
Q_{L}\left(x, x^{\prime}\right)=f\left(x^{\prime}\right)+\left(x-x^{\prime}\right) \nabla f\left(x^{\prime}\right)+\frac{L}{2}\left\|x-x^{\prime}\right\|^{2}+g\left(x^{\prime}\right),
$$

where $L$ is a Lipschitz constant of the gradient of $f$. The approximation can be minimized with proximal:

$$
p_{L}\left(x^{\prime}\right)=\operatorname{argmin}\left(Q_{L}\left(x, x^{\prime}\right)\right),
$$

which can be simplified to

$$
p_{L}\left(x^{\prime}\right)=\operatorname{argmin}\left(g(x)+\frac{L}{2}\left\|x-\left(x^{\prime}-\frac{1}{L} \nabla f\left(x^{\prime}\right)\right)\right\|^{2} .\right.
$$

The main iteration of the ISTA algorithm. Hence with $p_{L}$ given a Lipschitz constant of the gradient of $f$ and the last value $x_{i} \in \mathrm{R}^{2}$, the next value $x_{i+1}$ is calculated by:

$$
x_{i+1}=p_{L}\left(x_{i}\right) .
$$

The start value $x_{0}$ is initialized with an arbitrary value. We have now defined the IST algorithm.

ISTA can derived from different fields of mathematics [Bay16] . As outlined in [Fig09], it can be seen as a form of expectation maximization [NF01], as majorization minimization [BFO06] forwards backford splitting [LM79] [CW05] or as seperable approximation [WNF09].

Due to its simple structure, it is especially suited for dense linear inverse problems [BT09a]. However ISTA becomes slow if $A$ is ill-conditioned and the regularizer is small [FBA09] and according to[BT09a] the convergence of ISTA can be arbitrarily bad. Hence a number of improvements have been proposed for ISTA. Examples are TwISTA (two step ISTA) [BFO06] SpaRSA (sparse reconstruction by separable approximation) [WNF09], SALSA (split augmented Lagrangian shrinkage algorithm) [ABF10] and FISTA (fast iterative shrinkage thresholding algorithm) [BT09a].

In the following FISTA is introduced in more detail. This is a very short summary of the fast iterative shrinkage thresholding algorithm (FISTA), for more details see [BT09a], [Bub15]. Identical to the ISTA algorithm we first define the underlaying problem. Let $F: \mathbb{R}^{n} \rightarrow \mathbb{R}$ be a function with 
A. Mathematical and Physical Background

$F(x)=f(x)+g(x)$ and the optimization problem:

$$
\operatorname{argmin}_{x}(F(x)) \text {, }
$$

where $f: \mathbb{R}^{n} \rightarrow \mathbb{R}$ is a differentiable and convex function and $g: \mathbb{R}^{n} \rightarrow \mathbb{R}$ is non-smooth and convex.

We follow the definition of FISTA in [BT09b] and define $p_{L}$ as the proximal map of function $g$ :

$$
\begin{aligned}
p_{L}(y) & =\operatorname{prox}_{1 / L}(g)\left(y-\frac{1}{L} \nabla f(y)\right) \\
& =\operatorname{argmin}_{x}\left(\frac{L}{2}\left\|x-\left(y-\frac{1}{L} \nabla f(y)\right)\right\|^{2}+g(x)\right) .
\end{aligned}
$$

Given the upper bound Lipschitz constant $L(f)$ of $\nabla f$, initial value $y_{1}$ and $x_{0}$ and $t_{1}=1$, the FIST algorithm iteration is then defined as

$$
\begin{aligned}
x_{i} & =\operatorname{prox}_{1 / L}(g)\left(y-\frac{1}{L} \nabla f(y)\right) \\
t_{i+1} & =\frac{1+\sqrt{1+4 t_{i}^{2}}}{2} \\
y_{i+1} & =x_{i}+\frac{t_{i}-1}{t_{i+1}}\left(x_{i}-x_{i-1}\right) .
\end{aligned}
$$

This improved algorithm has a much better convergence then the original IST algorithm, which according to [BT09b] has a worst-case complexity of $O(1 / k)$, while the fast IST algorithm has a complexity of $O\left(1 / k^{2}\right)$. 


\section{A.8 Convolutional Neural Networks}

An artificial neural network is a structure of connected artificial neurons, that propagates signal from input to output depending on their configuration. In this section, we focus on convolutional neural networks as used for classification problems.

The name artificial neuronal network is chosen, because this structure partially mimics the behavior of real neurons [HW81], [LBB+98].

\section{A.8.1 Neuron}

The foundation of an neural network is the neuron. The artificial neuron is a mathematical model that models the behavior or reaction to an input configuration. It gives mapping between inputs value to an output value and can therefore be seen as a function. The neuron consists of a number $n \in \mathbb{N}$ of inputs indexed with $i$, which have values $x_{i} \in \mathbb{R}$. For each input there is a constant weight assigned $w_{i}$ that is multiplied with the respective input. The sum of the inputs multiplied by their weights is calculated and used for a function $\phi: \mathbb{R} \rightarrow \mathbb{R}$ that maps it to the output:

$$
y=\phi \sum_{i=0}^{n} x_{i} w_{i}
$$

One input, usually $x_{0}$, can be permanently set to 1 , so that the assigned weight acts as a bias $b$. The bias may be omitted, but systematic analysis of convolution neural network has shown that this decreases performance [MSM16].

Thus we can write:

$$
y=\phi\left(\sum_{i=1}^{n}\left(x_{i} w_{i}\right)+b\right) .
$$

Function $\phi$ is called the activation or transfer function, because it controls how the neuron reacts to an input level. There are numerous activation functions used in neural network. The inner composition of a neuron is highlighted in Figure A.9. It should be fast to calculate, differentiable, have a smooth derivate and include a non-linearity [NH10]. The most simple is a linear function, followed by the step function. The very commonly 
A. Mathematical and Physical Background

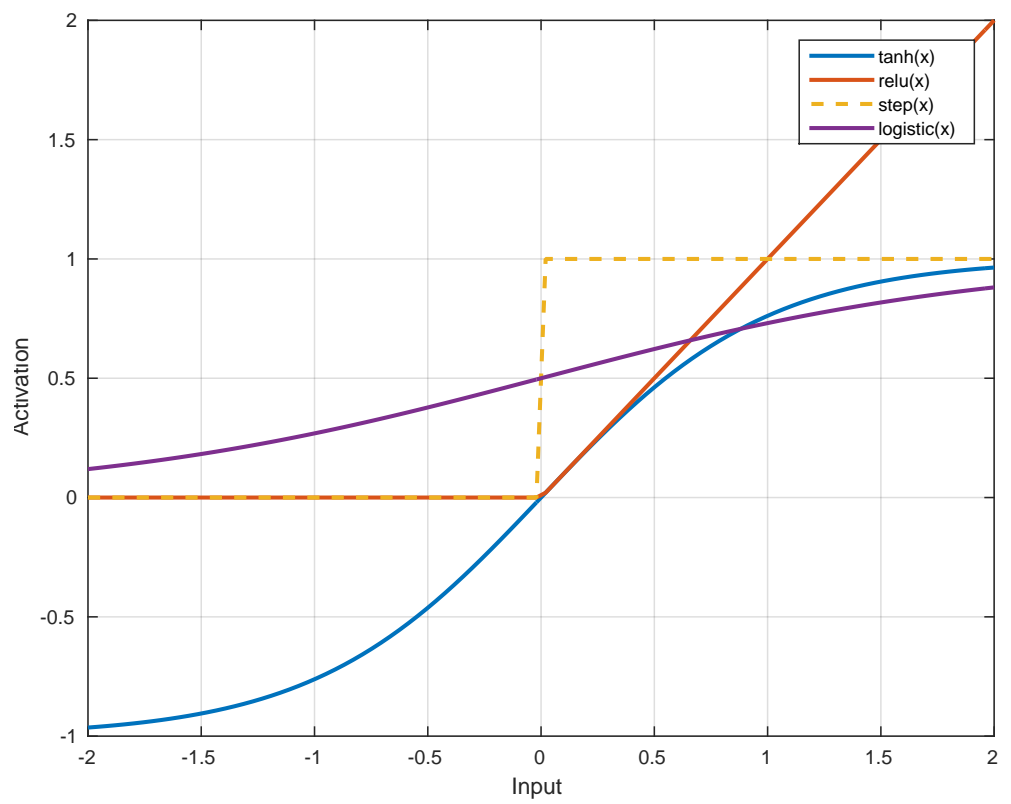

Figure A.8. Behavior of different activation functions.

used is also the tanh function. An alternative is the logistic function. The rectified linear unit ReLu activation function was invented in 2010 [NH10] is currently the most commonly used. [XWC+15]. With ReLu activation function, the value of a neuron is given by:

$$
y=\left\{\begin{array}{ll}
0 & \text { if } \sum_{i=1}^{n} x_{i} w_{i}+b<0 \\
\sum_{i=1}^{n} x_{i} w_{i}+b & \text { if } \sum_{i=1}^{n} x_{i} w_{i}+b \geqslant 0
\end{array} .\right.
$$

[MSM16] notes an increase in imagenet classification accuracy from tanh activation function to ReLu of approx. $3 \%$. 


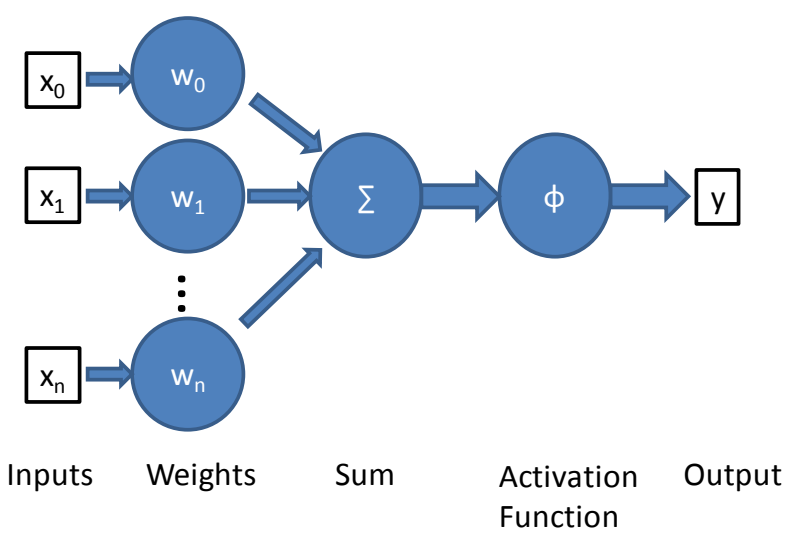

Figure A.9. Drawing of a neuron.

\section{A.8.2 MLP}

For neuronal networks the neurons introduced in the previous paragraph are arranged in layers. In three layer network as shown in Figure A.10, the output of the input layer neurons is the input for hidden layer neurons, whose output it the input of the output layer. The number of neurons in each layer is arbitrary and there may be several hidden layers in which the input of each layer is the set by output of the preceding layer. For a layer of neuron, organized as a vector, we now define a vector of inputs $x_{v}$, a vector of outputs $y_{v}$ and a matrix of weights $W$ and a vector of biases $b_{v}$. Such a configuration is commonly called the multilayer perceptron (MLP) [Ros62] and is a function mapping the configuration of the input vector $x_{v}$, i.e. the values set on the input layer, to an output vector $y_{v} \in \mathbb{R}^{m}$, i.e. the values of the output neurons:

$$
f_{m l p}: \mathbb{R}^{n} \rightarrow \mathbb{R}^{m}
$$

with

$$
y_{v}=f_{m l p}\left(x_{v}\right) .
$$

To generate the output of the neuronal network given an input configuration, the values of the neurons are propagated through the hidden 
A. Mathematical and Physical Background

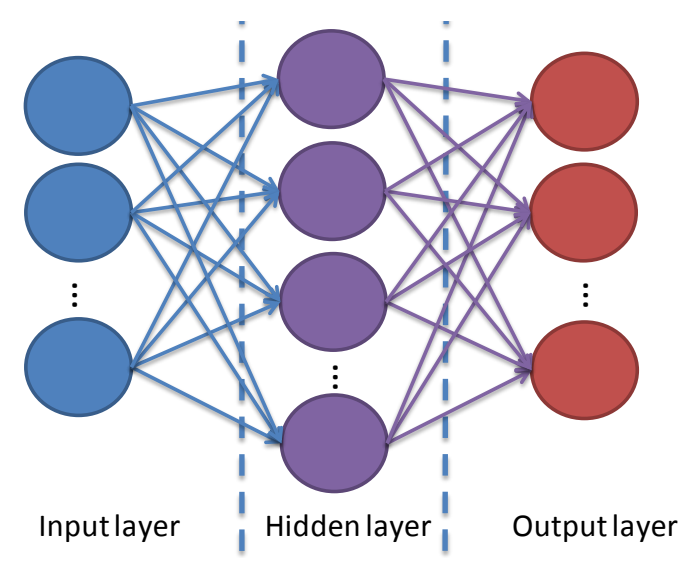

Figure A.10. Fully connected layers of neurons.

layers to the output layers. This process is called feed forward propagation or inference process of the neuronal network. The non-linearity of the activation function is important in this case. It can be shown that this is the requirement for the universal approximator called property of the MLP being able to express any non-pathological function.

\section{A.8.3 Convolutional Layers}

Convolutional layers are a variation of the multilevel perceptron in which the neurons of a layer share their weights $W$. Furthermore the number of weights is spatially restricted, so that neuron $i$ only has weights for input $\lfloor i-k / 2\rfloor$ and $\lceil i+k / 2\rceil$. The effect is that the layer is sensitive to spatial locality in the input data.

The application of a convolutional layer can now be seen as a convolution of the weight vector across the input:

$$
y_{v}=\phi\left(W \otimes x_{v}+b_{v}\right) .
$$

Because of this weight sharing the number of weights is significantly reduced, which is good for training the network but may restrict the network too strongly. Hence the concept of feature maps is introduced, 


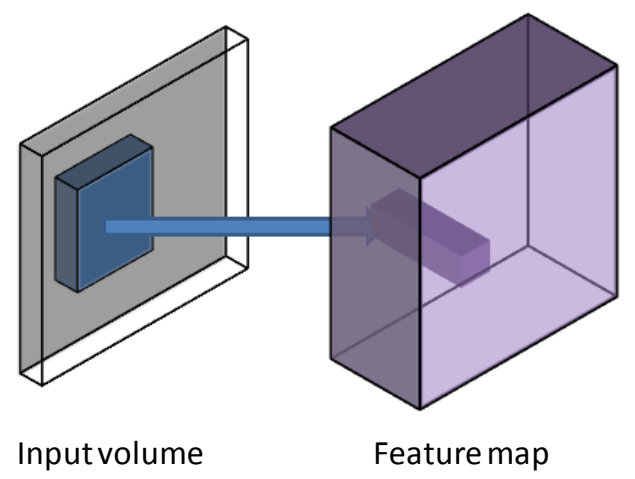

Figure A.11. Convolution resulting in 3D feature maps.

which are a collection of different weight configurations for one layer that give a give a collection of responses to one input.

If 2-dimensional data is used, the weights for each neuron also form a matrix, which then acts just like a convolution filter and the result is also 2-dimensional, hence the name convolutional layer. For the response to different weight configuration, the feature maps, now another dimension is needed, which is why they are typically shown as a $3 D$ volume. The concept of the feature map is illustrated in Figure A.11.

To further enforce the spatial locality of a signal and to further shrink the network, the response of a layer can be aggravated in a small section of the data. This aggregation process can be done with multiple strategies, such as replacing the section with the value maximum of a region or the mean value. The result is that less neurons can be used in the next layer without losing the important responses from the preceding layers.

A small convolutional network with two convolutional network with a pooling layer in between, followed by two fully connected layers is shown in Figure A.12. 


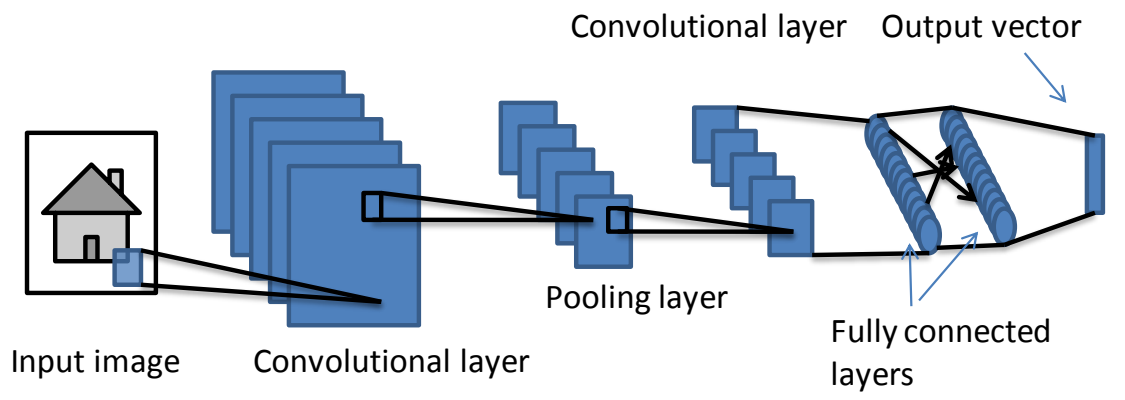

Figure A.12. A convolutional neuron network with two convolutional layers with a pooling layer in between, followed by two fully connected layers.

\section{A.8.4 Training}

Training is the process of optimizing the weights in a neural network so that the result of the neural network is optimal. Firstly we need to distinguish supervised and unsupervised training methods. Supervised training requires a training set with a known mapping of input to output, while with unsupervised training the network learns purely from observing the this input set [DKS+95]. Convolutional neural network are typically trained in a supervised way using a set of data called training data, which is composed of mappings between input and desired output.

Furthermore, a test dataset is defined which is distinct from training data, to be able to detect a loss of generality, called overfitting, which occurs, if the neural network has been tuned too much to the training data and would not respond positively to intended input data which is only similar to the training data. The performance of the neural network on this test dataset is typically used as a benchmark for the performance of the neural network overall.

Sometime another dataset is collected, the validation dataset. Typically while training a neural network, the network designer may want to change network parameters, like the networks depth, to boost the performance on the test dataset. The validation dataset can guard against overzealous tuning of such high-level parameters and is commonly used in compe- 
titions such as Imagenet [RDS+15], where it is only published after the competition ends. Tests against this dataset should only be done once, which is why the number of submissions is typically restricted.

To be able to optimize the weights of the network, we need to able to measure its performance. This is done with a loss function. This function employed to measure the difference between the current response of the neural network and the desired output. The choice depends on the problem to be solve. For a regression problem, where the output value of a neuron is measured, this is typically a least mean squares approach and for classification task, where the activation of a neuron is important, this can be a logistic loss function.

Optimization Algorithms Configuring the weights of the network so that its loss function produces low values is non-linear non-convex optimization problem. The main principle is that the weights are optimized stepwise from a given starting point. This starting point can be arbitrarily chosen e.g. to random values around zero. Gradient based algorithms are commonly used for this optimization task. Examples for these algorithms are stochastic gradient descent (SGD) [LBB+98] and Adam [KB14]. The step size of the optimization is controlled by the learning rate, which is a critical parameter for these type of optimization problems. Its importance comes from the high non-convexity of the optimization problem, which leads to many local minima that need to be avoided and the possibility of small or vanishing gradients, which leads to a slow optimization.

Backpropagation For the training of the network using optimization we need a strategy on how to adjust the weight of individual neurons. For this, a strategy called backpropagation $[R H W+88]$ is used. Backpropagation means that the value at the loss function is back propagated into the network to find out the contribution of a single neuron from the response of the entire network.

We assume that we have already computed the current activations with a forward propagation of a given input with the current best weights. Starting backwards, the chain rule of derivation is used recursively to calculate partial derivatives of different inputs, for details see [LBO+12] 


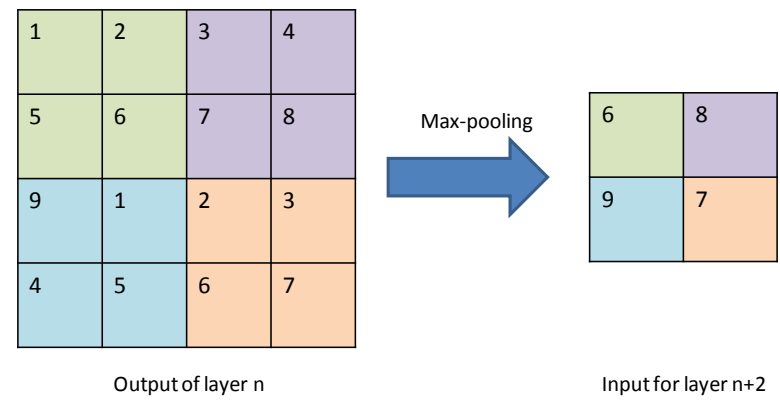

Figure A.13. Maximum pooling with a $2 \times 2$ block.

and algorithm 6.4 in [GBC16]. These partial derivatives mark the influence of the weights and therefore allow appropriate adjustment by the optimization algorithm.

Pooling A different layer often used as an intermediate layer between two convolutional layers are pooling layers. Pooling layers work as filters to concentrate the response of a layer in fewer neurons. The output of a previous layer may have arbitrary dimensions.

Now the pooling layer has a filter size of e.g. $2 \times 2$. This means for every block of $2 \times 2$ the maximum is calculated and used instead of this block. Note that no overlapping or folding occurs. This means that the size of the data is drastically reduced, which can reduce training time, memory footprint of the network and reduce overfitting.

For an example see Figure A.13, where such a filter is applied to a layer with $4 \times 4$ output elements. Different operators can be used to aggregate, the most common is the maximum function, leading to max-pooling. Maxpooling typically results in good performance, as seen in a comparison of pooling operations in [SMB10]. An alternative can be mean-pooling, which calculates the average of its block size.

This concludes the short introduction into convolutional networks. We can now construct arbitrary convolutional and fully connected networks and train them to solve different tasks, such as classification. 


\section{Bibliography}

[ABF10] Manya V. Afonso, José M. Bioucas-Dias, and Mário AT Figueiredo.

"Fast image recovery using variable splitting and constrained optimization". In: IEEE Transactions on Image Processing 19.9 (2010), pp. 2345-2356.

[Bay16] Ilker Bayram. "On the Convergence of the Iterative Shrinkage/Thresholding Algorithm With a Weakly Convex Penalty". In: IEEE Transactions on Signal Processing 64.6 (2016), pp. 15971608. DOI: $10.1109 /$ TSP. 2015.2502551.

[BCL02] Heinz H. Bauschke, Patrick L. Combettes, and D. Russell Luke. "Phase retrieval, error reduction algorithm, and Fienup variants: a view from convex optimization". In: JOSA A 19.7 (2002), pp. 1334-1345.

[BCL06] Heinz H. Bauschke, Patrick L. Combettes, and D. Russell Luke. "A strongly convergent reflection method for finding the projection onto the intersection of two closed convex sets in a Hilbert space". In: Journal of Approximation Theory 141.1 (2006), pp. 63-69. DOI: 10.1016/j. jat.2006.01.003.

[BCM+13] Afonso S. Bandeira, Jameson Cahill, Dustin G. Mixon, and Aaron A. Nelson. "Fundamental Limits of Phase Retrieval". In: Proc. 10th Intern. Conf. on Sampling Theory and Applications (SampTA). 2013.

[BCY+13] H. Bi, S. Cook, H. Yu, M. C. Benfield, and E. D. Houde. "Deployment of an imaging system to investigate fine-scale spatial distribution of early life stages of the ctenophore Mnemiopsis leidyi in Chesapeake Bay". In: Journal of Plankton Research 35.2 (2013), pp. 270-280. DOI: 10.1093/plankt/fbse94.

[BFO06] J.M. Bioucas-Dias, M.A.T. Figueiredo, and J.P. Oliveira. “Total Variation-Based Image Deconvolution: a Majorization- 
Bibliography

Minimization Approach". In: vol. 2. IEEE, 2006, pp. II-861-II864. ISBN: 978-1-4244-0469-8. DOI: 10.1109/ICASSP. 2006.1660479.

[BGB+15] Hongsheng Bi, Zhenhua Guo, Mark C. Benfield, Chunlei Fan, Michael Ford, Suzan Shahrestani, and Jeffery M. Sieracki. "A Semi-Automated Image Analysis Procedure for In Situ Plankton Imaging Systems". In: PLOS ONE 10.5 (2015). Ed. by Chih-hao Hsieh, e0127121. DOI: 10.1371/journal. pone. 0127121.

[Bi15] Hongsheng Bi. Replication Data for zooplankton image analysis DOI: PONE-d-14-43228. 2015. DOI: 10.7910/DVN/CLS5FW.

[BI16] Yuval Bahat and Michal Irani. "Blind dehazing using internal patch recurrence". In: 2016 IEEE International Conference on Computational Photography (ICCP). IEEE, 2016, pp. 1-9. DoI: 10.1109/ICСРнот.2016.7492870.

[BK15] Heinz H. Bauschke and Valentin R. Koch. "Projection methods: Swiss army knives for solving feasibility and best approximation problems with halfspaces". In: Contemporary Mathematics 636 (2015), pp. 1-40.

[Boa03] Boualem Boashash, ed. Time frequency signal analysis and processing: a comprehensive reference. 1st ed. Amsterdam ; Boston: Elsevier, 2003. ISBN: 978-0-08-044335-5.

[Bra00] Ronald N. Bracewell. The Fourier transform and its applications. 3rd ed. McGraw-Hill series in electrical and computer engineering. Boston: McGraw Hill, 2000.

[Bre17] Marianne Breinig. A Web-Enhanced Physics Course: Physics 421, Modern Optics, Module 5: Interference. The University of Tennessee. 2017. URL: http://electron6. phys. utk. edu/optics421/.

[BSZ+15] Liheng Bian, Jinli Suo, Guoan Zheng, Kaikai Guo, Feng Chen, and Qionghai Dai. "Fourier ptychographic reconstruction using Wirtinger flow optimization". In: Optics Express 23.4 (2015), p. 4856. DOI: 10.1364/0E.23.004856.

[BT09a] A. Beck and M. Teboulle. "Fast Gradient-Based Algorithms for Constrained Total Variation Image Denoising and Deblurring Problems". In: IEEE Transactions on Image Processing 18.11 (2009), pp. 2419-2434. DOI: 10.1109/TIP. 2009.2028250. 
[BT09b] Amir Beck and Marc Teboulle. "A Fast Iterative ShrinkageThresholding Algorithm for Linear Inverse Problems". In: SIAM Journal on Imaging Sciences 2.1 (2009), pp. 183-202. DOI: 10.1137/080716542.

[Bub15] Sébastien Bubeck. "Convex Optimization: Algorithms and Complexity". In: Foundations and Trends $®$ in Machine Learning 8.3-4 (2015), pp. 231-357. DOI: 10.1561/2200000050.

[BW01] Sven Bühling and Frank Wyrowski. "Solving tolerancing and three-dimensional beam shaping problems by multifunctional wave optical design". In: Optical Engineering 40.8 (2001), p. 1590 . DOI: $10.1117 / 1.1387278$.

[BW80] Max Born and Emil Wolf. Principles of optics: electromagnetic theory of propagation, interference and diffraction of light. 6th ed. Oxford ; New York: Pergamon Press, 1980. ISBN: 978-0-08026481-3.

[CCM+11] R Castrejón-García, JR Castrejón-Pita, GD Martin, and IM Hutchings. "The shadowgraph imaging technique and its modern application to fluid jets and drops". In: Revista mexicana de física 57.3 (2011), pp. 266-275.

[CCS+03] Raymond H. Chan, Tony F. Chan, Lixin Shen, and Zuowei Shen. "Wavelet algorithms for high-resolution image reconstruction". In: SIAM Journal on Scientific Computing 24.4 (2003), pp. 1408-1432.

[CEP+05] Tony Chan, Selim Esedoglu, Frederick Park, and A. Yip. "Recent developments in total variation image restoration". In: Mathematical Models of Computer Vision 17 (2005).

[CG08] Robert K. Cowen and Cedric M. Guigand. "In situ ichthyoplankton imaging system (ISIIS): system design and preliminary results". In: Limnol. Oceanogr. Methods 6.2 (2008), pp. 126-132. 
Bibliography

[CGG+13] Robert K Cowen, Adam T Greer, Cedric M Guigand, Jonathan A Hare, David E Richardson, and Harvey J Walsh. "Evaluation of the In Situ Ichthyoplankton Imaging System (ISIIS): comparison with the traditional (bongo net) sampler". In: Fishery Bulletin 111.1 (2013), pp. 1-12.

[CKC+06] Tristan Colomb, Jonas Kühn, Florian Charriere, Christian Depeursinge, Pierre Marquet, and Nicolas Aspert. "Total aberrations compensation in digital holographic microscopy with a reference conjugated hologram". In: Optics express 14.10 (2006), pp. 4300-4306.

[CLS15] Emmanuel J. Candes, Xiaodong Li, and Mahdi Soltanolkotabi. "Phase Retrieval via Wirtinger Flow: Theory and Algorithms". In: IEEE Transactions on Information Theory 61.4 (2015), pp. 19852007. DOI: 10.1109/TTT.2015.2399924.

[CMM+16] Lorenzo Corgnati, Simone Marini, Luca Mazzei, Ennio Ottaviani, Stefano Aliani, Alessandra Conversi, and Annalisa Griffa. "Looking inside the Ocean: Toward an Autonomous Imaging System for Monitoring Gelatinous Zooplankton". In: Sensors 16.12 (2016), p. 2124. DOI: 10.3390/s16122124.

[CP11] Antonin Chambolle and Thomas Pock. "A First-Order PrimalDual Algorithm for Convex Problems with Applications to Imaging". In: Journal of Mathematical Imaging and Vision 40.1 (2011), pp. 120-145. DOI: 10.1007/s10851-010-0251-1.

[CSR+15] Robert K Cowen, S Sponaugle, K Robinson, and J Luo. “Planktonset 1.0: Plankton imagery data collected from fg walton smith in straits of florida from 2014-06-03 to 2014-06-06 and used in the 2015 national data science bowl (ncei accession 0127422)". In: Oregon State University and Hatfield Marine Science Center (2015).

[CSV13] Emmanuel J. Candes, Thomas Strohmer, and Vladislav Voroninski. "Phaselift: Exact and stable signal recovery from magnitude measurements via convex programming". In: Communications on Pure and Applied Mathematics 66.8 (2013), pp. 12411274. 
[CW05] Patrick L Combettes and Valérie R Wajs. "Signal recovery by proximal forward-backward splitting". In: Multiscale Modeling E Simulation 4.4 (2005), pp. 1168-1200.

[CW98] Tony F. Chan and Chiu-Kwong Wong. "Total variation blind deconvolution". In: Image Processing, IEEE Transactions on 7.3 (1998), pp. 370-375.

[DKS+95] James Dougherty, Ron Kohavi, Mehran Sahami, et al. “Supervised and unsupervised discretization of continuous features". In: Machine learning: proceedings of the twelfth international conference. Vol. 12. 1995, pp. 194-202.

[DV90] P. Duhamel and M. Vetterli. "Fast fourier transforms: a tutorial review and a state of the art". In: Signal Processing 19.4 (1990), pp. 259-299. DOI: 10.1016/0165-1684(90)90158-u.

[EBY99] Gwynne A. Evans, Jonathan M. Blackledge, and Peter D. Yardley. Analytic Methods for Partial Differential Equations. Springer Undergraduate Mathematics Series. London: Springer London, 1999. DOI: 10.1007/978-1-4471-0379- 0 .

[ER11] René Escalante and Marcos Raydan. Alternating projection methods. Fundamentals of algorithms FA08. Philadelphia: Society for Industrial and Applied Mathematics, 2011. ISBN: 978-1-61197-193-4.

[Ers06] Okan K Ersoy. Diffraction, fourier optics and imaging. Vol. 30. John Wiley \& Sons, 2006.

[FBA09] Mario AT Figueiredo, Jose M. Bioucas-Dias, and Manya V. Afonso. "Fast frame-based image deconvolution using variable splitting and constrained optimization". In: 2009 IEEE/SP 15th Workshop on Statistical Signal Processing. IEEE, 2009, pp. 109-112.

[Fel76] Leopold B Felsen. “Evanescent waves”. In: JOSA 66.8 (1976), pp. 751-760.

[Fie06] Jim Fienup. "Phase Retrieval for Imaging and WaveFront Sensing". In: (2006). 
Bibliography

[Fie13] James R. Fienup. "Phase retrieval algorithms: a personal tour [invited]". In: Applied Optics 52.1 (2013), pp. 45-56.

[Fie78] James R. Fienup. "Reconstruction of an object from the modulus of its Fourier transform". In: Optics letters 3.1 (1978), pp. 27-29.

[Fie82] J. R. Fienup. "Phase retrieval algorithms: a comparison". In: Applied Optics 21.15 (1982), pp. 2758-2769. DoI: 10.1364/A0.21. 002758.

[Fie87] James R. Fienup. "Reconstruction of a complex-valued object from the modulus of its Fourier transform using a support constraint". In: JOSA A 4.1 (1987), pp. 118-123.

[Fig09] Mário A. T. Figueiredo. Iterative Shrinkage/Thresholding Algorithms: Some History and Recent Development. New York University, 2009. URL: apdio.pt/documents/10180/15233/Figueiredo_ IST2011. pdf.

[FJ04] Matteo Frigo and Steven G Johnson. "The FFTW web page". In: URL: http://www. fftw. org (2004).

[FJ12] Matteo Frigo and Steven G Johnson. "FFTW: Fastest Fourier transform in the west". In: Astrophysics Source Code Library (2012).

[FJ98] Matteo Frigo and Steven G Johnson. "FFTW: An adaptive software architecture for the FFT". In: Acoustics, Speech and Signal Processing, 1998. Proceedings of the 1998 IEEE International Conference on. Vol. 3. IEEE, 1998, pp. 1381-1384.

[FKJ+12] Konstantinos Falaggis, Tomasz Kozacki, Michal Jozwik, and Malgorzata Kujawinska. "Accurate and quantitative phase retrieval methods for a series of defocused images with application to in-line Gabor microscopy". In: SPIE Optical Engineering + Applications. International Society for Optics and Photonics, 2012, pp. 84930X-84930X. 
[FKK+14] Konstantinos Falaggis, Tomasz Kozacki, Malgorzata Kujawińska, Michał Józwik, and Arkadiusz Kuś. "High Precision Single Beam Phase Retrieval Techniques". In: Fringe 2013. Ed. by Wolfgang Osten. Berlin, Heidelberg: Springer Berlin Heidelberg, 2014, pp. 129-135. ISBN: 978-3-642-36359-7. DOI: $10.1007 / 978-3-642-36359-7 \_16$.

[FMC+03] Thierry Fusco, Laurent M. Mugnier, Jean-Marc Conan, Franck Marchis, G. Chauvin, Gerard Rousset, Anne-Marie Lagrange, David Mouillet, and Francois J. Roddier. "Deconvolution of astronomical images obtained from ground-based telescopes with adaptive optics". In: Proceedings of SPIE. Vol. 4839. 2003, pp. 1065-1075.

[FPL+16] Robin Faillettaz, Marc Picheral, Jessica Y. Luo, Cédric Guigand, Robert K. Cowen, and Jean-Olivier Irisson. "Imperfect automatic image classification successfully describes plankton distribution patterns". In: Methods in Oceanography 15-16 (2016), pp. 60-77. DOI: 10.1016/j.mio.2016.04.003.

[FSH+06] Rob Fergus, Barun Singh, Aaron Hertzmann, Sam T. Roweis, and William T. Freeman. "Removing camera shake from a single photograph". In: ACM Transactions on Graphics (TOG) 25.3 (2006), pp. 787-794.

[FWB+95] DA Fish, JG Walker, AM Brinicombe, and ER Pike. "Blind deconvolution by means of the Richardson-Lucy algorithm". In: JOSA A 12.1 (1995), pp. 58-65.

[FWW+14] Rong Fan, Qun Wan, Fei Wen, Hui Chen, and Yipeng Liu. "Iterative projection approach for phase retrieval of semisparse wave field". In: EURASIP Journal on Advances in Signal Processing 2014.1 (2014), pp. 1-13.

[GBC16] Ian Goodfellow, Yoshua Bengio, and Aaron Courville. Deep Learning. MIT Press, 2016.

[GCB+17] Michaël Gharbi, Jiawen Chen, Jonathan T. Barron, Samuel W. Hasinoff, and Frédo Durand. "Deep bilateral learning for real-time image enhancement". In: ACM Transactions on Graphics 36.4 (2017), pp. 1-12. DOI: 10.1145/3072959. 3073592. 
Bibliography

[GCG+15] Adam T Greer, Robert K Cowen, Cedric M Guigand, and Jonathan A Hare. "Fine-scale planktonic habitat partitioning at a shelf-slope front revealed by a high-resolution imaging system". In: Journal of Marine Systems 142 (2015), pp. 111-125.

[Get12] Pascal Getreuer. "Total Variation Deconvolution using Split Bregman". In: Image Processing On Line 2 (2012), pp. 158-174. DOI: 10.5201/ipol.2012.g-tvdc.

[GHH+13] James Gregson, Felix Heide, Matthias B. Hullin, Mushfiqur Rouf, and Wolfgang Heidrich. "Stochastic Deconvolution". In: IEEE, 2013, pp. 1043-1050. ISBN: 978-0-7695-4989-7. DOI: 10.1109/CVPR.2013.139.

[GJV+13] Michel Gross, Fadwa Joud, Frederic Verpillat, Max Lesaffre, and Nicolas Verrier. "Two-step distortion-free reconstruction scheme for holographic microscopy". In: OSA, 2013, DW1A.7. ISBN: 978-1-55752-964-0. DOI: 10.1364/DH.2013.DW1A.7.

[GL12] Bahadir Kursat Gunturk and Xin Li. Image Restoration: Fundamentals and Advances. CRC Press, 2012.

[GMC+15] P. Gaudio, A. Malizia, M. Camplani, F. Barbato, L. Antonelli, M. Gelfusa, M. Del Vecchio, L. Salgado, C. Bellecci, and M. Richetta. "Shadowgraph Technique Applied to STARDUST Facility for Dust Tracking: First Results". In: Physics Procedia 62 (2015), pp. 97-101. DOI: 10.1016/j. phpro.2015.02.017.

[GNM+16] J. D. Goodwin, E. W. North, I. D. Mitchell, C. M. Thompson, and H. R. McFadden. "Improving a semi-automated classification technique for bivalve larvae: Automated image acquisition and measures of quality control: Automated Image Acquisition of Bivalve Larvae". In: Limnology and Oceanography: Methods 14.11 (2016), pp. 683-697. DOI: 10.1002/lom3.10123.

[Goo05] Joseph W Goodman. Introduction to Fourier Optics. Roberts and Company Publishers, 2005.

[GS72] R. W. Gerchberg and W. O. Saxton. "A Practical Algorithm for the Determination of Phase from Image and Diffraction Plane Pictures". In: Optik 35.2 (1972), pp. 237-246. 
[GW06] Eric P. Goodwin and James C. Wyant. Field guide to interferometric optical testing. SPIE field guides v. FG10. Bellingham, Wash: SPIE Press, 2006. ISBN: 978-0-8194-6510-8.

[Hau68] Y. Haugazeau. Sur les inéquations variationnelles et la minimisation de fonctionnelles convexes. Thèse. Paris: Université de Paris, 1968.

[HDÁ10] Iris E Hendriks, Carlos M Duarte, and Marta Álvarez. “Vulnerability of marine biodiversity to ocean acidification: a meta-analysis". In: Estuarine, Coastal and Shelf Science 86.2 (2010), pp. 157-164.

[HG08] Quan Huynh-Thu and Mohammed Ghanbari. "Scope of validity of PSNR in image/video quality assessment". In: Electronics letters 44.13 (2008), pp. 800-801.

[HRH+13] Felix Heide, Mushfiqur Rouf, Matthias B. Hullin, Bjorn Labitzke, Wolfgang Heidrich, and Andreas Kolb. "High-quality computational imaging through simple lenses". In: $A C M$ Transactions on Graphics (TOG) 32.5 (2013), p. 149.

[HRR05] G Hays, A Richardson, and C Robinson. "Climate change and marine plankton". In: Trends in Ecology \& Evolution 20.6 (2005), pp. 337-344. DOI: 10.1016/j.tree.2005.03.004.

[HW81] DH Hubel and TN Wiesel. "Receptive Field and Functional Architecture fo Monkey Striate Cortex". In: From the Department of Physiology, Harvard Medical School: Molecular Processes in Vision 3 (1981), p. 382.

[HWL+00] Roger Harris, Peter Wiebe, Jurgen Lenz, Hein-Rune Skjoldal, and Mark Huntley. ICES zooplankton methodology manual. Academic press, 2000.

[HZ10] Alain Hore and Djemel Ziou. "Image quality metrics: PSNR vs. SSIM". In: Pattern recognition (icpr), 2010 20th international conference on. IEEE, 2010, pp. 2366-2369.

[HZR+16] Kaiming He, Xiangyu Zhang, Shaoqing Ren, and Jian Sun. "Deep Residual Learning for Image Recognition". In: IEEE, 2016, pp. 770-778. ISBN: 978-1-4673-8851-1. DOI: 10.1109/CVPR. 2016. 90. 
Bibliography

[IK01] Laurent Itti and Christof Koch. "Computational modelling of visual attention". In: Nature reviews. Neuroscience 2.3 (2001), p. 194.

[Jäh02] Bernd Jähne. Digitale Bildverarbeitung. Berlin, Heidelberg: Springer Berlin Heidelberg, 2002. ISBN: 978-3-662-06732-1. DOI: 10.1007/978-3-662-06731-4.

[Jan12] Peter A. Jansson, ed. Deconvolution of images and spectra. 2nd ed. Dover ed. Mineola, N.Y: Dover Publications, 2012.

[JEH15] Kishore Jaganathan, Yonina C. Eldar, and Babak Hassibi. "Phase retrieval: An overview of recent developments". In: arXiv preprint arXiv:1510.07713 (2015).

[JSD+14] Yangqing Jia, Evan Shelhamer, Jeff Donahue, Sergey Karayev, Jonathan Long, Ross Girshick, Sergio Guadarrama, and Trevor Darrell. "Caffe: Convolutional Architecture for Fast Feature Embedding". In: arXiv:1408.5093 [cs] (2014).

[Kam00] David W. Kammler. A first course in Fourier analysis. Upper Saddle River, NJ: Prentice Hall, 2000. ISBN: 978-0-13-578782-3.

[KB14] Diederik Kingma and Jimmy Ba. "Adam: a method for stochastic optimization". In: arXiv preprint arXiv:1412.6980 (2014).

[KDL13] Abhishek Kumar, Wolfgang Drexler, and Rainer A. Leitgeb. "Subaperture correlation based digital adaptive optics for full field optical coherence tomography". In: Optics Express 21.9 (2013), p. 10850. DOI: 10.1364/0E.21.010850.

[KF09] Dilip Krishnan and Rob Fergus. "Fast Image Deconvolution using Hyper-Laplacian Priors". In: Advances in Neural Information Processing Systems 22. Ed. by Y. Bengio, D. Schuurmans, J. D. Lafferty, C. K. I. Williams, and A. Culotta. Curran Associates, Inc., 2009, pp. 1033-1041.

[KS10] Joseph Katz and Jian Sheng. "Applications of Holography in Fluid Mechanics and Particle Dynamics". In: Annual Review of Fluid Mechanics 42.1 (2010), pp. 531-555. DoI: 10.1146/annurevfluid-121108- 145508. 
[KSH12] Alex Krizhevsky, Ilya Sutskever, and Geoffrey E Hinton. "Imagenet classification with deep convolutional neural networks". In: Advances in neural information processing systems. 2012, pp. 1097-1105.

[KŠM13] Jan Kotera, Filip Šroubek, and Peyman Milanfar. “Blind deconvolution using alternating maximum a posteriori estimation with heavy-tailed priors". In: Computer Analysis of Images and Patterns. Springer, 2013, pp. 59-66.

[LBB+98] Y. Lecun, L. Bottou, Y. Bengio, and P. Haffner. "Gradientbased learning applied to document recognition". In: Proceedings of the IEEE 86.11 (1998), pp. 2278-2324.

[LBO+12] Yann A. LeCun, Léon Bottou, Genevieve B. Orr, and KlausRobert Müller. "Efficient BackProp". In: Neural Networks: Tricks of the Trade: Second Edition. Ed. by Grégoire Montavon, Geneviève B. Orr, and Klaus-Robert Müller. Berlin, Heidelberg: Springer Berlin Heidelberg, 2012, pp. 9-48. DOI: 10.1007/ 978- 3-642-35289-8_3.

[LF15] Tatiana Latychevskaia and Hans-Werner Fink. "Practical algorithms for simulation and reconstruction of digital in-line holograms". In: Applied Optics 54.9 (2015), p. 2424. DoI: 10.1364/ A0.54.002424.

[LFD+07] Anat Levin, Rob Fergus, Fredo Durand, and William T. Freeman. "Deconvolution using natural image priors". In: Massachusetts Institute of Technology, Computer Science and Artificial Intelligence Laboratory (2007).

[LIP+16] Liat Liberman, Yonatan Israel, Eilon Poem, and Yaron Silberberg. "Quantum enhanced phase retrieval". In: Optica 3.2 (2016), p. 193. DOI: 10.1364/0PTICA.3.000193.

[LM79] Pierre-Louis Lions and Bertrand Mercier. "Splitting algorithms for the sum of two nonlinear operators". In: SIAM Journal on Numerical Analysis 16.6 (1979), pp. 964-979. 
Bibliography

[LMH+15] Dongming Li, Gai Mengye, Chen Haochuan, Zhu Guang, and Lijuan Zhang. "Research on wavefront correction algorithm of Adaptive Optics system". In: Computer Science and Network Technology (ICCSNT), 2015 4th International Conference on. Vol. 1. IEEE, 2015, pp. 23-27.

[LS83] Aharon Levi and Henry Stark. "Signal restoration from phase by projections onto convex sets". In: Journal of the Optical Society of America 73.6 (1983), p. 810. DOI: 10.1364/J0SA.73.000810.

[LSY+17] Dongming Li, Changming Sun, Jinhua Yang, Huan Liu, Jiaqi Peng, and Lijuan Zhang. "Robust Multi-Frame Adaptive Optics Image Restoration Algorithm Using Maximum Likelihood Estimation with Poisson Statistics". In: Sensors 17.4 (2017), p. 785. DOI: 10.3390/s17040785.

[Luc74] L. B. Lucy. "An iterative technique for the rectification of observed distributions". In: The Astronomical Journal 79 (1974), p. 745. DOI: 10.1086/111605.

[Luk05] D Russell Luke. "Relaxed averaged alternating reflections for diffraction imaging". In: Inverse Problems 21.1 (2005), pp. 3750. DOI: 10.1088/0266-5611/21/1/004.

[LWD+09] Anat Levin, Yair Weiss, Fredo Durand, and William T. Freeman. "Understanding and evaluating blind deconvolution algorithms". In: Computer Vision and Pattern Recognition, 2009. CVPR 2009. IEEE Conference on. IEEE, 2009, pp. 1964-1971.

[LWD+11] A. Levin, Y. Weiss, F. Durand, and W. T. Freeman. "Understanding Blind Deconvolution Algorithms". In: IEEE Transactions on Pattern Analysis and Machine Intelligence 33.12 (2011), pp. 2354-2367. DOI: 10.1109/TPAMI.2011.148.

[LYI+13] Dhugal Lindsay, Hiroshi Yoshida, Shojiro Ishibashi, Mitsuko Umetsu, Atsushi Yamaguchi, Hiroyuki Yamamoto, Jun Nishikawa, James Davis Reimer, Hiromi Watanabe, Katsunori Fujikura, et al. "The uROV PICASSO, the Visual Plankton Recorder, and other attempts to image plankton". In: Underwater Technology Symposium (UT), 2013 IEEE International. IEEE, 2013, pp. 1-3. 
[LYZ10] Zijia Li, Zhengfeng Yang, and Lihong Zhi. "Blind image deconvolution via fast approximate GCD". In: Proceedings of the 2010 International Symposium on Symbolic and Algebraic Computation. ACM, 2010, pp. 155-162.

[Mar07a] S. Marchesini. "A unified evaluation of iterative projection algorithms for phase retrieval". In: Review of Scientific Instruments 78.1 (2007), p. 011301. DOI: 10.1063/1.2403783.

[Mar07b] S. Marchesini. "Invited Article: a unified evaluation of iterative projection algorithms for phase retrieval". In: Review of Scientific Instruments 78.1 (2007), p. 011301. DoI: 10.1063/1.2403783.

[Mat15] Matlab. Version 8.5 (r2015a). Natick, Massachusetts: The MathWorks Inc., 2015.

[Maz13] Amrita Mazumdar. "Principles and techniques of schlieren imaging systems". In: Columbia University Computer Science Technical Reports (2013).

[MCM+15] Simone Marini, Lorenzo Corgnati, Luca Mazzei, Ennio Ottaviano, Bruno Isoppo, Stefano Aliani, Alessandra Conversi, and Annalisa Griffa. "GUARD1: An autonomous system for gelatinous zooplankton image-based recognition". In: OCEANS 2015-Genova. IEEE, 2015, pp. 1-7.

[MCN+12] Sam McClatchie, Robert Cowen, Karen Nieto, Adam Greer, Jessica Y. Luo, Cedric Guigand, David Demer, David Griffith, and Daniel Rudnick. "Resolution of fine biological structure including small narcomedusae across a front in the Southern California Bight". In: Journal of Geophysical Research: Oceans 117.C4 (2012), n/a-n/a. DOI: 10.1029/2011Jc007565.

[Mer88] F Merkle. "Aktive und adaptive Optik in der Astronomie: Neue Technologien für zukünftige Grossteleskope". In: Physikalische Blätter 44.12 (1988), pp. 439-446.

[MFC04] Laurent M. Mugnier, Thierry Fusco, and Jean-Marc Conan. "MISTRAL: a myopic edge-preserving image restoration method, with application to astronomical adaptive-opticscorrected long-exposure images". In: JOSA A 21.10 (2004), pp. 1841-1854. 
Bibliography

[MI14] Tomer Michaeli and Michal Irani. “Blind Deblurring Using Internal Patch Recurrence". In: Computer Vision-ECCV 2014. Springer, 2014, pp. 783-798.

[MKS+09] Joseph C. Marron, Richard L. Kendrick, Nathan Seldomridge, Taylor D. Grow, and Thomas A. Höft. "Atmospheric turbulence correction using digital holographic detection: experimental results". In: Optics express 17.14 (2009), pp. 1163811651.

[MOK+16] Noriaki Miura, Akira Oh-ishi, Susumu Kuwamura, Naoshi Baba, Satoru Ueno, Yoshikazu Nakatani, and Kiyoshi Ichimoto. "Deconvolution of partially compensated solar images from additional wavefront sensing". In: Applied Optics 55.10 (2016), p. 2484. DOI: 10.1364/A0.55.002484.

[MRC+01] Laurent M. Mugnier, Clélia Robert, Jean-Marc Conan, Vincent Michau, and Sélim Salem. "Myopic deconvolution from wavefront sensing". In: JOSA A 18.4 (2001), pp. 862-872.

[MRC+99] Laurent M. Mugnier, Clelia Robert, Jean-Marc Conan, Vincent Michau, and S. Salem. "Regularized multiframe myopic deconvolution from wavefront sensing". In: ed. by Michael C. Roggemann and Luc R. Bissonnette. 1999, pp. 134-144. DoI: 10.1117/12.363607.

[MS14] Mehdi Molaei and Jian Sheng. "Imaging bacterial 3d motion using digital in-line holographic microscopy and correlationbased de-noising algorithm". In: Optics Express 22.26 (2014), p. 32119. DOI: 10.1364/0E.22. 032119 .

[MSM16] Dmytro Mishkin, Nikolay Sergievskiy, and Jiri Matas. "Systematic evaluation of CNN advances on the ImageNet". In: arXiv preprint arXiv:1606.02228 (2016).

[MXV14] Hashim Mir, Peter Xu, and Peter Van Beek. “An extensive empirical evaluation of focus measures for digital photography". In: ISET/SPIE Electronic Imaging. International Society for Optics and Photonics, 2014, pp. 90230I-90230I. 
[Neu51] John von Neumann. Functional Operators (AM-22), Volume 2: The Geometry of Orthogonal Spaces. (AM-22). Princeton: Princeton University Press, 1951. IsBN: 978-1-4008-8225-0.

[NF01] Robert D Nowak and Mário AT Figueiredo. "Fast waveletbased image deconvolution using the EM algorithm". In: Signals, Systems and Computers, 2001. Conference Record of the Thirty-Fifth Asilomar Conference on. Vol. 1. IEEE, 2001, pp. 371375.

[NH10] Vinod Nair and Geoffrey E Hinton. "Rectified linear units improve restricted boltzmann machines". In: Proceedings of the 27th international conference on machine learning (ICML-10). 2010, pp. 807-814.

[NJS15] Praneeth Netrapalli, Prateek Jain, and Sujay Sanghavi. "Phase Retrieval Using Alternating Minimization". In: IEEE Transactions on Signal Processing 63.18 (2015), pp. 4814-4826. DOI: 10.1109/TSP. 2015.2448516.

[Nol76] Robert J. Noll. "Zernike polynomials and atmospheric turbulence". In: JOsA 66.3 (1976), pp. 207-211.

[OFA+05] James C Orr, Victoria J Fabry, Olivier Aumont, Laurent Bopp, Scott C Doney, Richard A Feely, Anand Gnanadesikan, Nicolas Gruber, Akio Ishida, Fortunat Joos, et al. "Anthropogenic ocean acidification over the twenty-first century and its impact on calcifying organisms". In: Nature 437.7059 (2005), pp. 681-686.

[OP94] Arkadiusz Orlowski and Harry Paul. "Phase retrieval in quantum mechanics". In: Physical Review A 50.2 (1994), R921R924. DOI: 10.1103/PhysRevA. 50.R921.

[Osh12] Eliyahu Osherovich. "Numerical methods for phase retrieval". In: arXiv preprint arXiv:1203.4756 (2012).

[Ots79] Nobuyuki Otsu. "A Threshold Selection Method from GrayLevel Histograms". In: IEEE Transactions on Systems, Man, and Cybernetics 9.1 (1979), pp. 62-66. DOI: 10.1109/TSMc. 1979.4310076.

[Pas08] Rüdiger Paschotta. Encyclopedia of laser physics and technology. Weinheim: Wiley-VCH, 2008. IsBN: 978-3-527-40828-3. 
Bibliography

[PF14] Daniele Perrone and Paolo Favaro. "Total variation blind deconvolution: The devil is in the details". In: 2014 IEEE Conference on Computer Vision and Pattern Recognition. IEEE, 2014, pp. 2909-2916.

[POZ05] Giancarlo Pedrini, Wolfgang Osten, and Yan Zhang. “Wavefront reconstruction from a sequence of interferograms recorded at different planes". In: Optics Letters 30.8 (2005), p. 833. Dor: 10.1364/0L. 30. 000833 .

[PRF90] J. Primot, G. Rousset, and J. C. Fontanella. “Deconvolution from wave-front sensing: a new technique for compensating turbulence-degraded images". In: JOSA A 7.9 (1990), pp. 1598-1608.

[RDS+15] Olga Russakovsky et al. "ImageNet Large Scale Visual Recognition Challenge". In: International Journal of Computer Vision (IJCV) 115.3 (2015), pp. 211-252. DOI: 10.1007/s11263-015-0816-y.

[RHK+13] Chelsea M Rochman, Eunha Hoh, Tomofumi Kurobe, and Swee J Teh. "Ingested plastic transfers hazardous chemicals to fish and induces hepatic stress". In: Scientific reports 3 (2013).

[RHW+88] David E Rumelhart, Geoffrey E Hinton, Ronald J Williams, et al. "Learning representations by back-propagating errors". In: Cognitive modeling 5.3 (1988), p. 1.

[Ric08] Anthony J Richardson. "In hot water: zooplankton and climate change". In: ICES Journal of Marine Science 65.3 (2008), pp. 279-295.

[Ric72] William Hadley Richardson. "Bayesian-based iterative method of image restoration". In: JOSA 62.1 (1972), pp. 55-59.

[RMZ12] M. Rostami, O. Michailovich, and Zhou Wang. “Image Deblurring Using Derivative Compressed Sensing for Optical Imaging Application". In: IEEE Transactions on Image Processing 21.7 (2012), pp. 3139-3149. DOI: 10.1109/TIP.2012.2190610. 
[Roc15] Chelsea M Rochman. "The complex mixture, fate and toxicity of chemicals associated with plastic debris in the marine environment". In: Marine anthropogenic litter. Springer, 2015, pp. 117-140.

[Ros62] F. Rosenblatt. Principles of neurodynamics: perceptrons and the theory of brain mechanisms. Report (Cornell Aeronautical Laboratory). Spartan Books, 1962.

[RP89] George O. Reynolds and George B. Parrent, eds. The New physical optics notebook: tutorials in Fourier optics. Bellingham, Wash., USA: SPIE Optical Engineering Press, 1989.

[RSW+13] Julia Reisser, Jeremy Shaw, Chris Wilcox, Britta Denise Hardesty, Maira Proietti, Michele Thums, and Charitha Pattiaratchi. "Marine plastic pollution in waters around Australia: characteristics, concentrations, and pathways". In: PloS one 8.11 (2013), e80466.

[SBE14] Yoav Shechtman, Amir Beck, and Yonina C. Eldar. "GESPAR: Efficient Phase Retrieval of Sparse Signals". In: IEEE Transactions on Signal Processing 62.4 (2014), pp. 928-938. Dor: 10.1109/TSP. 2013.2297687.

[SBH+13] Christian J. Schuler, Harold Christopher Burger, Stefan Harmeling, and Bernhard Scholkopf. "A Machine Learning Approach for Non-blind Image Deconvolution". In: IEEE, 2013, pp. 1067-1074. ISBN: 978-0-7695-4989-7. DOI: 10.1109/CVPR.2013.142.

[SBP14] Igor A. Shevkunov, Nikolay S. Balbekin, and Nikolay V. Petrov. "Comparison of digital holography and iterative phase retrieval methods for wavefront reconstruction". In: Proc. SPIE 9271, Holography, Diffractive Optics, and Applications VI. Vol. 9271. 2014, pp. 927128-927128-9. DOI: 10.1117/12.2071469.

[Sch93] Timothy J. Schulz. "Multiframe blind deconvolution of astronomical images". In: JOSA A 10.5 (1993), pp. 1064-1073.

[SDN04] Yu Sun, Stefan Duthaler, and Bradley J. Nelson. "Autofocusing in computer microscopy: selecting the optimal focus algorithm". In: Microscopy research and technique 65.3 (2004), pp. 139-149. 
Bibliography

[SEC+15] Yoav Shechtman, Yonina C. Eldar, Oren Cohen, Henry Nicholas Chapman, Jianwei Miao, and Mordechai Segev. "Phase Retrieval with Application to Optical Imaging: a contemporary overview". In: IEEE Signal Processing Magazine 32.3 (2015), pp. 87-109. DOI: 10.1109/MSP.2014.2352673.

[Set01] G. S. Settles. Schlieren and Shadowgraph Techniques. Berlin, Heidelberg: Springer Berlin Heidelberg, 2001. ISBN: 978-3642-63034-7. DOI: 10.1007/978-3-642-56640-0.

[Set17] Gary S. Settles. "Smartphone schlieren and shadowgraph imaging". In: Optics and Lasers in Engineering (2017). DOI: 10.1016/j . optlaseng. 2017.07.002.

[SFC09] Filip Sroubek, Jan Flusser, and Gabriel Cristóbal. "Superresolution and blind deconvolution for rational factors with an application to color images". In: Computer Journal 52.1 (2009), p. 142.

[SFM+11] Emilio Sánchez-Ortiga, Pietro Ferraro, Manuel MartínezCorral, Genaro Saavedra, and Ana Doblas. "Digital holographic microscopy with pure-optical spherical phase compensation". In: JOSA A 28.7 (2011), pp. 1410-1417.

[SI07] Eli Shechtman and Michal Irani. "Matching Local Self-Similarities across Images and Videos". In: 2007 IEEE Conference on Computer Vision and Pattern Recognition. IEEE, 2007, pp. 1-8. DOI: 10.1109/CVPR. 2007.383198.

[SLJ+15] Christian Szegedy, Wei Liu, Yangqing Jia, Pierre Sermanet, Scott Reed, Dragomir Anguelov, Dumitru Erhan, Vincent Vanhoucke, and Andrew Rabinovich. "Going Deeper with Convolutions". In: Computer Vision and Pattern Recognition (CVPR). 2015.

[SMB10] Dominik Scherer, Andreas Müller, and Sven Behnke. “Evaluation of pooling operations in convolutional architectures for object recognition". In: Artificial Neural Networks-ICANN 2010 (2010), pp. 92-101. 
[Soi13] V. A. Soifer, ed. Computer design of diffractive optics. Woodhead publishing series in electronic and optical materials number 50. Oxford ; Philadelphia: Woodhead Publishing : CISP, 2013. ISBN: 978-1-84569-635-1.

[SPD+09] Chandra Sekhar Seelamantula, Nicolas Pavillon, Christian Depeursinge, and Michael Unser. "Zero-order-free image reconstruction in digital holographic microscopy". In: Biomedical Imaging: From Nano to Macro, 2009. ISBI'09. IEEE International Symposium on. IEEE, 2009, pp. 201-204.

[SS15] Basty Ajay Shenoy and Chandra Sekhar Seelamantula. "Exact Phase Retrieval for a Class of 2-d Parametric Signals". In: IEEE Transactions on Signal Processing 63.1 (2015), pp. 90-103. DOI: 10.1109/TSP. 2014.2370935.

[STS+13] Fatemeh Saki, Amir Tahmasbi, Hamid Soltanian-Zadeh, and Shahriar B. Shokouhi. "Fast opposite weight learning rules with application in breast cancer diagnosis". In: Computers in Biology and Medicine 43.1 (2013), pp. 32-41. DoI: 10.1016/j. compbiomed.2012.10.006.

[TMB05] Mark V. Trevorrow, David L. Mackas, and Mark C. Benfield. "Comparison of multifrequency acoustic and in situ measurements of zooplankton abundances in Knight Inlet, British Columbia". In: The Journal of the Acoustical Society of America 117.6 (2005), pp. 3574-3588. Dor: 10.1121/1.1920087.

[TSS11] Amir Tahmasbi, Fatemeh Saki, and Shahriar B. Shokouhi. "Classification of benign and malignant masses based on Zernike moments". In: Computers in Biology and Medicine 41.8 (2011), pp. 726-735. DOI: 10.1016/j. compbiomed.2011.06.009.

[VBD+05] R. Vio, J. Bardsley, M. Donatelli, and W. Wamsteker. "Dealing with edge effects in least-squares image deconvolution problems". In: Astronomy \& Astrophysics 442.1 (2005), pp. 397-403. DOI: $10.1051 / 0004-6361: 20053414$. 
Bibliography

[VMS+11] Jean Vernin et al. "European Extremely Large Telescope Site Characterization i: Overview". In: Publications of the Astronomical Society of the Pacific 123.909 (2011), pp. 1334-1346. Dor: 10. 1086/662995.

[WBS+04] Zhou Wang, Alan C Bovik, Hamid R Sheikh, and Eero P Simoncelli. "Image quality assessment: from error visibility to structural similarity". In: IEEE transactions on image processing 13.4 (2004), pp. 600-612.

[Wie13] Norbert Wiener. Extrapolation, interpolation, and smoothing of stationary time series: with engineering applications. Martino Fine Books, 2013. ISBN: 978-1-61427-517-6.

[WNF09] Stephen J Wright, Robert D Nowak, and Mário AT Figueiredo. "Sparse reconstruction by separable approximation". In: IEEE Transactions on Signal Processing 57.7 (2009), pp. 2479-2493.

[WYH08] Tao Wang, Zhaohua Yin, and Wenrui Hu. “On a combined measurement technique of PIV and shadowgraph in environmental fluid dynamics". In: Proceedings of the SPIE. Ed. by Xiaoyuan He, Huimin Xie, and YiLan Kang. 2008, 73751Q. DOI: $10.1117 / 12.839070$.

[XRL+14] Li Xu, Jimmy SJ Ren, Ce Liu, and Jiaya Jia. “Deep convolutional neural network for image deconvolution". In: Advances in Neural Information Processing Systems. 2014, pp. 1790-1798.

[XWC+15] Bing Xu, Naiyan Wang, Tianqi Chen, and Mu Li. "Empirical Evaluation of Rectified Activations in Convolutional Network". In: CoRR abs/1505.00853 (2015).

[YK75] K. H. Yang and J. D. Kingsley. "Calculation of Coupling Losses Between Light Emitting Diodes and Low-Loss Optical Fibers". In: Applied Optics 14.2 (1975), p. 288. DoI: 10.1364/A0.14. 000288 .

[YLG+92] Jeannette Yen, Petra H. Lenz, Donald V. Gassie, and Daniel K. Hartline. "Mechanoreception in marine copepods: electrophysiological studies on the first antennae". In: Journal of Plankton Research 14.4 (1992), pp. 495-512. 
[YRW08] J. Yen, K.D. Rasberry, and D.R. Webster. "Quantifying copepod kinematics in a laboratory turbulence apparatus". In: Journal of Marine Systems 69.3-4 (2008), pp. 283-294. DOI: 10.1016/j.jmarsys. 2006.02.014.

[YW82] Dan C. Youla and Heywood Webb. "Image Restoration by the Method of Convex Projections: Part 1 theory". In: Medical Imaging, IEEE Transactions on 1.2 (1982), pp. 81-94.

[ZK16] Claudius Zelenka and Reinhard Koch. "Restoration of images with wavefront aberrations". In: Pattern Recognition (ICPR), 2016 23rd International Conference on. IEEE, 2016, pp. 13881393.

[ZK17] Claudius Zelenka and Reinhard Koch. “Improved wavefront correction for coherent image restoration". In: Optics Express 25.16 (2017), p. 18797. DOI: 10.1364/0E.25.018797.

[ZM10] Christiane Zarfl and Michael Matthies. "Are marine plastic particles transport vectors for organic pollutants to the Arctic?" In: Marine Pollution Bulletin 60.10 (2010), pp. 18101814. 



\section{List of Symbols}

W General wavefront deformation. 32, 33

$O_{a}$ Image plane amplitude. 22, 58-60

$P_{A}$ Projection onto the set of points where the aperture plane constraints hold. 24, 25

$P_{F}$ Projection onto the set of points where the (virtual) focal plane constraints hold. 38, 39, 41, 42, 48, 49

$P_{o}$ Projection onto the set of points where the image plane constraints hold. 24, 25, 38, 39, 41, 42, 48, 49

$P_{f S S}$ Projection onto the set of self similar images. 49

$V_{o}$ Set of points where the object-space constraints hold. 24

$W_{f}$ Transformation matrix between image and focus plane. 43-46

$W$ Transformation matrix between image and focus plane. 43, 46

$\phi_{d}$ Phase delay of a thin lens. 33, 59, 60

$\phi_{m}$ Multiplicative phase delay term of a thin lens. 33, 35

$\phi_{p}$ Phase delay due to wavefront propagation. 33

$\phi_{s}$ Multiplicative phase delay term of an aberration used for correction in WFC algorithms. 35, 40, 43, 49

$\phi_{s a}$ Phase delay of an aberration used in WFC-FISTA. 43

$f_{w f c}$ First part of WFC-FISTA target function. 45, 46

$g_{w f_{c}}$ Second part of WFC-FISTA target function. 45

O Image plane. $22-25,35,36,38-41,43-46,48,49,58$ 
List of Symbols

$A_{m}$ Disturbed complex image amplitude. 7

A Aperture plane (pupil plane). 22, 23, 35, 40, 43, 59

$B_{a}$ Complex amplitude blur kernel. 7

$B$ Incoherent blur kernel. 6, 7, 15

E Error against first subscript plane constraints in second subscript iteration. 40,41

F (Virtual) Focal plane. 35, 40, 41, 50, 51

$\mathcal{F}$ Fourier transform. 17, 23, 35, 49, 59, 60, 153, 155, 156

$\mathbb{F}$ Fourier transform left multiplication matrix. 43

$I_{\text {inc }}$ Undisturbed intensity image. 6, 15, 17, 58, 59, 161

$I_{r}$ Restored image. 17, 59, 60, 161

$\lambda$ Wavelength used in simulations. 62, 65, 66, 68, 117

$N_{a}$ Amplitude noise. 7

$N$ Image acquisition noise. 6, 15

$O_{\text {dis }}$ Disturbed complex image plane. 59

$O_{S C}$ Image sensor intensity measurements with coherent image formation. 59,60

$O_{S I}$ Incoherent disturbed image intensity. 6, 7, 15, 59, 60

$R$ Radial polynomial used in Zernike polynomials. 162, 163

$r$ Radius of a thin lens. 33, 36

Re Real part function. 46, 146 
$R_{f}$ Reflection with the set of points where the (virtual) focal plane constraints hold. 39

$R_{o}$ Reflection with the set of points where the image plane constraints hold. 39

OTF Optical transfer function. 17

PSF Point spread function. 59, 60

$U$ Complex image amplitude. 7

Z Zernike polynomial with single or double index. 33, 64, 65, 162, 164 



\section{List of Symbols - POCS \& CNN}

H Hilbert space. 166, 167

P Projection onto a set. 166

Q Haugazeau helper function. 39, 167

$R$ Reflection projection. 166

$T$ Operator used in HAAR projection algorithm. 39, 167

$W$ Weight matrix of a neuron network layer. 173, 174

$\phi$ Activation function. 171, 174

$b_{v}$ Bias vector of a neuron network layer. 173, 174

$b \quad$ Bias value of a neuron. 171, 172

$f_{m l p}$ Function of an MLP. 173

$w_{i}$ Weight of an input $i$ of a neuron. 171, 172

$w$ Weight vector of a neuron.

$x_{i}$ Input value $i$ of a neuron. 171, 172

$x_{v}$ Weight vector of a neuron. 173, 174

$y_{v}$ Weight vector of a neuron. 173, 174

y Output value of a neuron. 171, 172 



\section{List of Symbols - Scalar Diffraction Theory}

A Angular spectrum. 147-150

$B$ Magnetic flux density. 145

c Speed of light. 145,158

$\delta_{f}$ breath of the frequency spectrum. 158

E Electric field. 144, 145

$\epsilon_{0}$ Permittivity of free space. 144,145

$\epsilon$ Permittivity. 144, 145

$f$ Focal length. 152-156

$H$ Magnetic field. 144, 145

$H$ Angular spectrum transfer function. 150

$H_{f}$ Angular spectrum propagation transfer function with Fresnel approximation. 151, 153, 155

$h_{f}$ Fresnel diffraction impulse response. 151, 153, 154

k Wave number. 23, 33, 36, 146, 148, 151-154

$k_{z}$ Wave vector in z-direction. 148-150

$k_{z}^{\prime}$ Approximation of wave vector in z-direction. 151 
List of Symbols - Scalar Diffraction Theory

$\lambda$ Wave length. 33, 146-151, 154-156

$L_{c o h}$ breath of the frequency spectrum. 158

$\mu_{0}$ Magnetic permeability of free space. 144,145

$\mu$ Magnetic permeability. 144

$n$ Refractive index. 143, 145, 152

$v$ Frequency. 146, 158

$\omega$ Angular speed. 146

$\Phi$ scalar of wave field. 146

$\phi$ Phase. 17, 22, 58, 59, 146

t Time. 144, 145, 158

$t_{c o h}$ breath of the frequency spectrum. 158

U Complex wavefield. 147-149, 153-156

$w$ Wave vector. 147,148 


\section{List of Abbreviations}

ANSI

$\mathrm{AO}$

BFGS

$\mathrm{CNN}$

CPU

DCT

FFT

FISTA

GPU

GS

HAAR

$\mathrm{HIO}$

$\mathrm{HIO}$

ISIIS

IST

KCSS

L-BFGS

LED
American national standards institute. adaptive optics.

Broyden Fletcher Goldfarb Shannon algorithm.

Convolutional neural network.

Central processing unit.

Discrete cosine transform.

Fast Fourier transform.

Fast iterative shrinkage thresholding algorithm.

Graphics processing unit.

Gerchberg-Saxton algorithm.

Haugazeau alternating averaged projections algorithm.

Average projections algorithm.

Hybrid-Input-Output algorithm.

In situ ichtyoplankton imaging system.

Iterative shrinkage thresholding algorithm.

Kiel computer science series.

Limited memory-Broyden Fletcher Goldfarb Shannon algorithm.

Light emitting diode. 
List of Abbreviations

\begin{tabular}{|c|c|}
\hline MAP & Multiple alternating projections algorithm. \\
\hline MAP & Maximum a posteriori. \\
\hline MI & Initialization with blurred image. \\
\hline ML & Maximum likelihood. \\
\hline MLP & Multi layer perceptron. \\
\hline MP & Mega pixel. \\
\hline MSE & Mean squared error. \\
\hline OTF & Optical transfer function. \\
\hline PIV & Particle image velocimetry. \\
\hline POCS & Projections onto convex sets. \\
\hline PSF & Point spread function. \\
\hline PSNR & Peak signal to noise ratio. \\
\hline RAAR & $\begin{array}{l}\text { Reflective alternating averaged reflections algo- } \\
\text { rithm. }\end{array}$ \\
\hline ReLu & rectified linear unit. \\
\hline RI & Initialization with random values. \\
\hline RL & Richardson-Lucy algorithm. \\
\hline RMS & Root mean square. \\
\hline SBMIR & Single-Beam-Multiple-Intensity Reconstruction. \\
\hline SGD & Stochastic gradient descent. \\
\hline WFC & $\begin{array}{l}\text { Wavefront Correction - Gerchberg Saxton algo- } \\
\text { rithm. }\end{array}$ \\
\hline WFC & Wavefront Correction algorithm. \\
\hline WFC-AP & $\begin{array}{l}\text { Wavefront Correction - Average projections al- } \\
\text { gorithm. }\end{array}$ \\
\hline WFC-HAAR & $\begin{array}{l}\text { Wavefront Correction - Haugazeau alternating } \\
\text { averaged projections algorithm. }\end{array}$ \\
\hline WFC-HIO & $\begin{array}{l}\text { Wavefront Correction - Hybrid-Input-Output } \\
\text { algorithm. }\end{array}$ \\
\hline WFC-RAAR & $\begin{array}{l}\text { Wavefront Correction - Reflective alternating } \\
\text { averaged reflections algorithm. }\end{array}$ \\
\hline
\end{tabular}


WFC-SS Wavefront Correction - Self similarity algorithm.

ZI Initialization to zero. 


